\title{
Numerische Untersuchung rotierender Rayleigh-Bénard-Konvektion ohne Ekman-Schichten
}

\author{
Dissertation \\ zur Erlangung des Doktorgrades \\ der Mathematisch-Naturwissenschaftlichen Fakultäten \\ der Georg-August-Universität zu Göttingen
}

vorgelegt von

Simon Schmitz

aus

Bad Neustadt a. d. Saale

Göttingen 2009 
Referent:

Korreferent:

Tag der mündlichen Prüfung: $\quad$ 16. November 2009 


\section{Inhaltsverzeichnis}

$\begin{array}{lll}\text { Nomenklatur } & \text { III }\end{array}$

1 Einleitung 1

2 Rayleigh-Bénard-Konvektion $\quad 5$

2.1 Das Rayleigh-Bénard-System . . . . . . . . . . . . . . . . . 5

2.2 Die Gleichungen des Systems . . . . . . . . . . . . . . . . . 6

2.2.1 Entdimensionalisierte Gleichungen . . . . . . . . . . . . . . . 8

2.2.2 Überblick über die Kennzahlen . . . . . . . . . . . . . . . . . . . 8

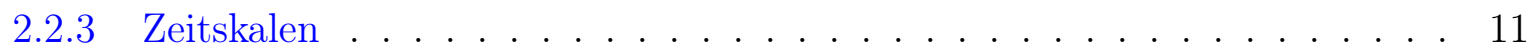

2.3 Lineare Stabilitätsanalyse für verschiedene Ekman-Zahlen . . . . . . . . . . . . . 12

3 Numerische Umsetzung $\quad 15$

3.1 Behandlung der Navier-Stokes-Gleichung und der Temperaturgleichung . . . . . 15

3.1.1 Behandlung der Navier-Stokes-Gleichung: Aufspaltung der Geschwindigkeit 15

3.1.2 Behandlung der Temperaturgleichung . . . . . . . . . . . . . . 17

3.2 Räumliche und zeitliche Diskretisierung . . . . . . . . . . . . . . . . 18

3.2.1 Zerlegung nach Fourier-Koeffizienten . . . . . . . . . . . . 18

3.2.2 Zerlegung nach Chebyshev-Koeffizienten . . . . . . . . . . . . 19

3.2.3 Zeitliche Diskretisierung . . . . . . . . . . . . . . 21

3.3 Beispiel: Gleichungssystem für Poloidal- und Toroidalfeld . . . . . . . . . . . . . 22

3.3.1 Expliziter Coriolisterm . . . . . . . . . . . . . . 22 
3.3 .2 Impliziter Coriolisterm . . . . . . . . . . . . . . . . . . . . . . 24

4 Beschreibung des Programms $\quad 27$

4.1 Änderungen am Programm . . . . . . . . . . . . . . . . . . . . . . 27

4.2 Aufbau des Programms . . . . . . . . . . . . . . . . . . . . . . . 28

4.3 Parallelisierung und Speicheraufteilung . . . . . . . . . . . . . . 30

4.4 Berechnung der expliziten rechten Seiten . . . . . . . . . . . . . . . . 32

$\begin{array}{lll}5 & \text { Ergebnisse } & 35\end{array}$

5.1 Geschwindigkeitsgrenzschichten . . . . . . . . . . . . . . 36

5.2 Reynolds-Zahl . . . . . . . . . . . . . . . . . . . . . . . . . . 39

5.3 Nusselt-Zahl . . . . . . . . . . . . . . . . . . . . . . . . . . . 39

5.3.1 Nusselt-Zahl ohne Rotation . . . . . . . . . . . . . . . . . . . . . 41

5.3 .2 Nusselt-Zahl mit Rotation . . . . . . . . . . . . . . . . . . . . . . . 44

5.3 .3 Diffusionsfreie Kennzahlen . . . . . . . . . . . . . . . . . . . . 53

5.4 Strömungsstrukturen . . . . . . . . . . . . . . . . . 57

5.4 .1 Helizität der Strömung . . . . . . . . . . . . . . . . . 57

5.4 .2 Spektren des konvektiven Wärmetransports . . . . . . . . . . . . 60

5.5 Diskussion der Ergebnisse . . . . . . . . . . . . . . . . . . 76

$\begin{array}{llr}6 & \text { Zusammenfassung } & 79\end{array}$

$\begin{array}{lr}\text { A Tabellen der Simulationsergebnisse } & 81\end{array}$

$\begin{array}{ll}\text { B Chebychev-Polynome } & 91\end{array}$

$\begin{array}{lr}\text { C Kinetische Energiedissipation } & 93\end{array}$

$\begin{array}{lr}\text { Literaturverzeichnis } & 97\end{array}$

$\begin{array}{ll}\text { Abbildungsverzeichnis } & 103\end{array}$

$\begin{array}{ll}\text { Tabellenverzeichnis } & 107\end{array}$ 


\section{Nomenklatur}

\begin{tabular}{|c|c|}
\hline $\mathbf{x}$ & Kartesische Koordinaten: $\mathbf{x}=(x, y, z)$ \\
\hline$\partial_{x}, \partial_{y}, \partial_{z}$ & Partielle räumliche Ableitung \\
\hline$\langle\ldots\rangle_{x, y}$ & Horizontales Mittel über Ebenen mit konstantem z \\
\hline$\langle\ldots\rangle_{V}$ & Räumliches Mittel über das gesamte Simulationsgebiet \\
\hline$\hat{\mathbf{z}}$ & Einheitsvektor in $z$-Richtung \\
\hline$t$ & Zeit \\
\hline$\partial_{t}$ & Partielle zeitliche Ableitung \\
\hline$\langle\ldots\rangle_{t}$ & Zeitliches Mittel über die gesamte Simulationsdauer \\
\hline $\mathbf{u}$ & Geschwindigkeitsfeld: $\mathbf{u}=\left(u_{x}, u_{y}, u_{z}\right)$ \\
\hline$u_{h}$ & Horizontale Geschwindigkeit: $u_{h}=\sqrt{u_{x}^{2}+u_{y}^{2}}$ \\
\hline$\delta_{u}$ & Geschwindigkeitsgrenzschicht \\
\hline$\Phi$ & Poloidalfeld der Geschwindigkeit \\
\hline$\Psi$ & Toroidalfeld der Geschwindigkeit \\
\hline $\mathrm{U}$ & Geschwindigkeit des Meanflows: $\mathbf{U}=\left(U_{x}, U_{y}\right)$ \\
\hline $\mathrm{g}$ & Vektor der Schwerebeschleunigung: $g=|\mathbf{g}|$ \\
\hline$\Omega$ & Vektor der Winkelgeschwindigkeit (Corioliskraft): $\Omega=|\Omega|$ \\
\hline k & Horizontaler Wellenvektor: $\mathbf{k}=\left(k_{x}, k_{y}\right), k=|\mathbf{k}|$ \\
\hline$\lambda$ & Horizontale Wellenlänge der Strömung \\
\hline$\lambda_{c}$ & Kritische horizontale Wellenlänge der Strömung (am Onset) \\
\hline$\lambda_{m}$ & Mittlere (repräsentative) Wellenlänge \\
\hline$D$ & Spektrale Dichte \\
\hline
\end{tabular}


T

$\Theta$

$\Delta T$

$T_{s}$

$\delta_{T}$

$p$

$\pi$

d

L

$\Gamma$

$\varrho$

$\nu$

$\kappa$

l

$\alpha$

Q

$R a$

$R a_{c}$

$\mathrm{Pr}$

$E k$

Ta

$\mathrm{Nu}$

Re

$R e_{\text {rot }}$

$P e$

$P e_{\text {rot }}$

Ro

$R a_{*}$

$N u_{*}$

$R a_{f *}$
Temperatur

Temperaturvariation

Temperaturdifferenz zwischen der oberen und unteren Platte

Statisches Temperaturprofil

Temperaturgrenzschicht

Druck

Druckvariation

Höhe der Zelle

Länge bzw. Breite der Zelle

Aspektverhältnis: $\Gamma=\frac{L}{d}$

Dichte

Kinematische Viskosität

Thermische Diffusivität

Thermische Leitfähigkeit

Thermischer Volumenausdehnungskoeffizient

Charakteristischer Wärmefluss

Rayleigh-Zahl: $R a=\frac{g \alpha d^{3} \Delta T}{\nu \kappa}$

Kritische Rayleigh-Zahl (am Onset)

Prandtl-Zahl: $\operatorname{Pr}=\frac{\nu}{\kappa}$

Ekman-Zahl: $E k=\frac{\nu}{\Omega D^{2}}$

Taylor-Zahl: $T a=4 E k^{-2}=\frac{4 \Omega^{2} D^{4}}{\nu^{2}}$

Nusselt-Zahl: $N u=\frac{Q D}{l \Delta T}$

Reynolds-Zahl: $R e=\frac{U D}{\nu}$

Rotations-Reynolds-Zahl: $R e=\frac{U}{\nu} \sqrt{\frac{\nu}{\Omega}}=R e E k^{1 / 2}$

Péclet-Zahl: $P e=\frac{U D}{\kappa}=R e P r$

Rotations-Péclet-Zahl: $P e_{\text {rot }}=\frac{U}{\kappa} \sqrt{\frac{\nu}{\Omega}}=\operatorname{Re} \operatorname{Pr} E k^{1 / 2}$

Rossby-Zahl: $R o=\frac{U}{\Omega D}=R e E k$

Diffusionsfreie Rayleigh-Zahl: $R a_{*}=\frac{g \alpha \Delta T}{\Omega^{2} d}=R a E k^{2} \operatorname{Pr}^{-1}$

Diffusionsfreie Nusselt-Zahl: $N u_{*}=\frac{Q}{\varrho c_{p} \Delta T \Omega d}=N u E k \operatorname{Pr}^{-1}$

Diffusionsfreie Fluss-Rayleigh-Zahl: $R a_{f *}=\frac{g \alpha Q}{\varrho c_{p} \Omega^{3} d^{2}}=R a_{*} N u_{*}=R a E k^{3} \operatorname{Pr}^{-2}$ 



\section{Kapitel 1}

\section{Einleitung}

Konvektionsströmungen treten in der Natur sehr häufig auf. In der Atmosphäre führt die Aufheizung durch Sonneneinstrahlung dazu, dass sich Konvektionszellen über z.B. warmen Seegebieten/Ozeanen bilden. Dies ist in Abbildung 1.1, rechts, dargestellt. Im Ozean selbst ist die thermohaline Konvektion verantwortlich für die Ozeanströmung, der Golfstrom ist das beste Beispiel dafür. Und im flüssigen äußeren Erdkern schließlich sind Konvektionsströmungen wichtig für die Erzeugung des Magnetfeldes der Erde.
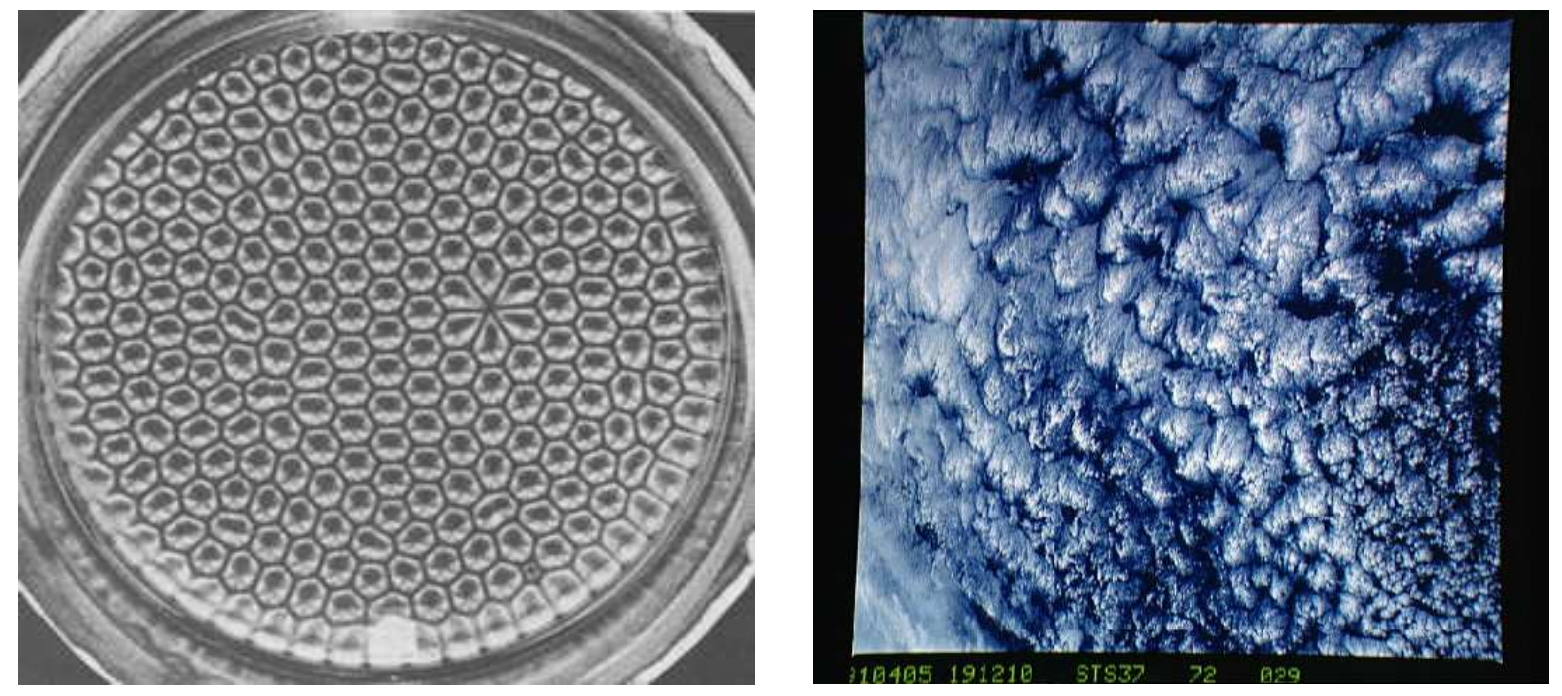

Abb. 1.1: Konvektionszellen bei Rayleigh-Bénard-Konvektion. Links: Bénard-Zellen in einer dünnen Flüssigkeitsschicht (aus [1]). Rechts: Konvektionszellen über dem Pazifik (aus [2]).

Bei der Untersuchung thermischer Konvektion werden zum Beispiel der Wärmetransport oder die Strukturen eines thermisch instabilen Fluids betrachtet. Ein Fluid ist thermisch instabil, wenn sich kaltes (schweres) über warmem (leichtem) Fluid befindet. Das warme Fluid erfährt 
Kapitel 1. Einleitung

durch seine geringere Dichte einen Auftrieb, entgegengesetzt zur Gravitationskraft. Unterhalb einer kritischen Temperaturdifferenz bzw. einer kritischen Rayleigh-Zahl (die eine dimensionslose Kennzahl für die Temperaturdifferenz ist) wird Wärme nur durch Konduktion transportiert. Ab der kritischen Rayleigh-Zahl setzt die Konvektionsströmung ein. Diese ist zuerst laminar und wird mit steigender Rayleigh-Zahl turbulenter.

Untersuchungen zur Konvektion gehen zurück auf Henry Bénard zu Beginn des 20. Jahrhunderts (siehe [6]). In seinem Experiment (siehe Abbildung 1.1, links) wird eine dünne Fluidschicht von unten beheizt und es bilden sich Konvektionszellen aus. Allerdings stellte es sich später heraus, dass nicht die thermische Instabilität für diese Muster verantwortlich war, sondern vor allem die Oberflächenspannung, die sich mit der Temperatur ändert.

Der Einfluss der Corioliskraft wurde schon früh (z.B. 1969 von Rossby, siehe [33]) untersucht. Sie führt dazu, dass die Konvektion erst ab einer größeren kritischen Rayleigh-Zahl einsetzt, da sie durch die Rotation unterdrückt wird. Für Werte wenig oberhalb dieser kritischen RayleighZahl ist die Strömung durch die Rotation bestimmt. Erst für viel größere Rayleigh-Zahlen wird die Rotation unwichtig und die Strömung verhält sich wieder wie im Fall ohne Rotation.

Diese Dissertation beschäftigt sich mit der Untersuchung thermischer Rayleigh-BénardKonvektion unter dem Einfluss der Rotation mittels direkter numerischer Simulationen. Dazu wurde eine dreidimensionale ebene horizontale Schicht eines Fluids (charakterisiert durch die dimensionslose Prandtl-Zahl) simuliert, bei der der Vektor der Gravitationsbeschleunigung in negative $z$-Richtung zeigt. Der Vektor der Winkelgeschwindigkeit ist antiparallel dazu orientiert. Ihr Betrag bestimmt die Größe der Ekman-Zahl, einer dimensionslosen Kennzahl für die Corioliskraft. Diese Ekman-Zahl ist neben Rayleigh- und Prandtl-Zahl der Hauptparamter der Strömung.

Untersucht wurde der Wärmetransport in Abhängigkeit der drei genannten Kennzahlen. Dabei wurde eine Auftragung über einer Rotations-Péclet-Zahl $P e_{\text {rot }}$ gefunden, bei der der Wärmetransport für Rechnungen mit unterschiedlichen Kennzahlen zu einer einzigen Kurve zusammenfallen. Anhand der Rotations-Péclet-Zahl kann also ein Kriterium dafür definiert werden, ab wann der Wärmetransport nicht mehr von der Rotation beeinflusst ist. In dieser Auftragung kann man zusätzlich zu den rotationsdominierten und rotationsfreien Regimes einen Übergangsbereich identifizieren. Für diesen Übergangsbereich liefert eine Betrachtung diffusionsfreier Kennzahlen ein Potenzgesetz, das gut mit anderen Simulationen bzw. Experimenten übereinstimmt.

Daneben wurde auch die normierte Helizität untersucht, um zu entscheiden, ab wann die Strömung selbst nicht mehr durch die Rotation bestimmt wird. Dies lässt sich anhand der Betrach- 
tung der kinetischen Energiedissipation untermauern. Hierbei ist die Rotations-Reynolds-Zahl $R e_{\text {rot }}$ das Kriterium, das die Grenze zum nichtrotierenden Bereich festlegt.

Schließlich wurde die Größe der entstandenen Strömungsstrukturen betrachtet. Diese werden für den rotationsfreien und rotationsbehafteten Fall gegenübergestellt. Eine Auftragung der aus den Spektren gewonnenen mittleren Wellenlängen zeigt in einer Auftragung über $P e_{\text {rot }}$ ebenfalls nur eine einzige Kurve.

Die Arbeit gliedert sich in mehrere Kapitel. Nach dieser Einleitung werden in Kapitel 2 die Grundlagen erklärt. Dazu zählen die physikalischen und entdimensionalisierten Gleichungen sowie die Kennzahlen. Auch ein Test des Programms mittels linearer Stabilitätsanalyse wird diskutiert.

In Kapitel 3 wird die Umsetzung der Gleichungen des vorigen Kapitels beschrieben. Dabei wird besonders auf die Diskretisierung und die Aufstellung der Matrizen des Gleichungssystems eingegangen. Anhand eines Beispiels wird die Implementierung der Randbedingungen erklärt. Kapitel 4 geht näher auf den Aufbau des Programms selbst und den Aspekt der Parallelisierung ein. Auch der Berechnung der expliziten rechten Seiten des Gleichungssystems ist ein Abschnitt gewidmet.

Kapitel 5 beinhaltet die Ergebnisse der Arbeit. Zusätzlich zu den Simulationen mit schubspannungsfreien Rändern werden auch die Ergebnisse einiger Simulationen mit no-slip Rändern vorgestellt. Zuerst wird der Unterschied zwischen den beiden Randbedingungen anhand von Geschwindigkeitsprofilen und deren Grenzschichten hervorgehoben. Dann wird der Wärmetransport untersucht. Dabei wird vor allem auf die Auftragung über $P e_{r o t}$ eingegangen, um ein Kriterium zu finden, ab wann der Wärmetransport nicht mehr durch die Rotation beeinflusst wird. Ebenfalls wird die Helizität der Strömung ausgewertet, als Maß für den Einfluss der Rotation auf die Struktur des Fluids. Daraus kann wiederum ein Kriterium für den Übergang zum rotationsfreien Regime hergeleitet werden, das nur von der Rotations-Reynolds-Zahl $R e_{r o t}$ abhängt. Die Größe der Strömungsstrukturen wird durch Betrachtung der vorherrschenden Wellenlängen und der Spektren ausgewertet.

Zum Schluss folgen die Zusammenfassung in Kapitel 6 und mehrere Anhänge. In diesen sind die Tabellen der Simulationsergebnisse (Anhang A), die Eigenschaften der Chebychev-Polynome (Anhang B) und die Herleitung der kinetischen Energiedissipation (Anhang C) enthalten.

Die Ergebnisse der Arbeit wurden veröffentlicht in [35]. 



\section{Kapitel 2}

\section{Rayleigh-Bénard-Konvektion}

In diesem Kapitel wird das Rayleigh-Bénard-System vorgestellt. Zuerst wird der Aufbau des Simulationsgebietes besprochen. Danach werden die Gleichungen, die das System beschreiben, vorgestellt und entdimensionalisiert. Die dabei entstehenden Kennzahlen werden am Beispiel des Erdkerns in den Bezug zur Realität gesetzt. Schließlich wird noch ein Testfall für das Programm, nämlich eine lineare Stabilitätsanalyse zur Bestimmung des Onsets für verschiedene Rotationsraten, diskutiert.

\subsection{Das Rayleigh-Bénard-System}

Die Rayleigh-Bénard-Konvektion wird in der vorliegenden Arbeit in einem quaderförmigen Gebiet simuliert. Der Quader ist charakterisiert durch das Aspektverhältnis $\Gamma$, welches das Verhältnis der Länge bzw. Breite zur Höhe angibt. Bei den Simulationen wurde immer eine quadratische Grundfläche verwendet, so dass $\Gamma=\Gamma_{x}=\Gamma_{y}=L / d$ ist. Das Fluid wird in vertikaler Richtung durch zwei Platten begrenzt. Diese haben unterschiedliche Temperaturen $T_{1}$ und $T_{2}$ und den Abstand $d$ voneinander, siehe Abbildung 2.1. Dabei ist die Temperatur an der unteren Platte größer als an der oberen, damit ein instabiler Temperaturgradient entsteht.

Die Platten sind aber keine festen Wände in dem Sinn, dass alle Geschwindigkeitskomponenten verschwinden müssen. Es gilt also nicht die no-slip Bedingung, wie man es im Laborexperiment vorfände. An den Platten sind schubspannungsfreie Randbedingungen implementiert, die nur dafür sorgen, dass die z-Komponente der Geschwindigkeit verschwindet. Für die horizontalen Geschwindigkeitskomponenten sollen lediglich die Ableitungen in z-Richtung verschwinden (siehe Kapitel 3, Abschnitt 3.3 für ein Beispiel). In horizontaler Richtung soll möglichst kein 
Einfluss durch Seitenwände entstehen, es werden deshalb periodische Randbedingungen verwendet. Die Fluidschicht ist so ausgerichtet, dass der Vektor der Schwerebeschleunigung $\mathbf{g}$

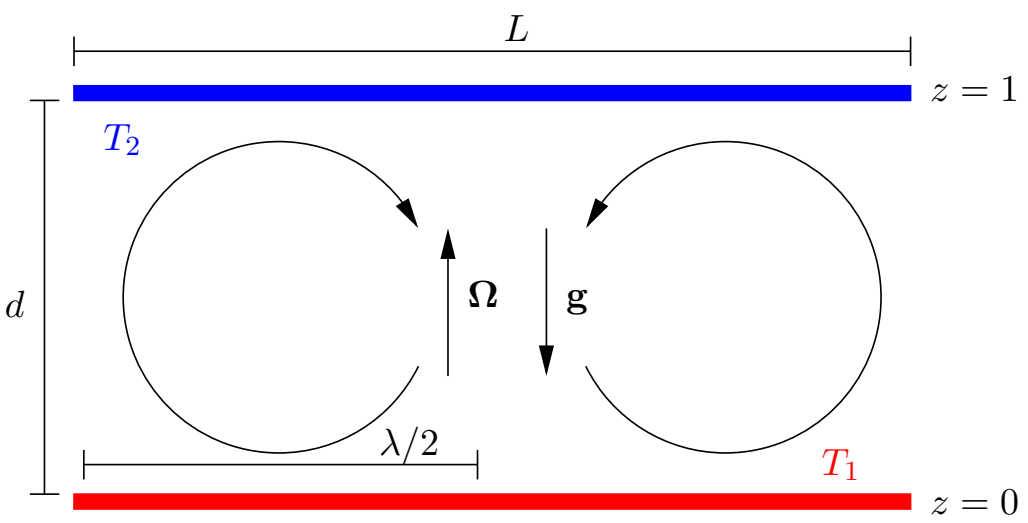

Abb. 2.1: Aufbau des Simulationsgebiets mit Abmessungen. Schematisch eingezeichnet sind zwei Konvektionsrollen.

senkrecht nach unten zeigt. Der Vektor der Winkelgeschwindigkeit $\Omega$ zeigt in entgegengesetzte Richtung. Das Fluid selbst wird als inkompressibel angenommen. Dies ist möglich, solange die Temperaturunterschiede nicht zu groß sind.

\subsection{Die Gleichungen des Systems}

Dieses System mit einem inkompressiblen Fluid lässt sich mathematisch durch drei Gleichungen beschreiben:

$$
\begin{aligned}
\varrho \partial_{t} \mathbf{u}+\varrho(\mathbf{u} \cdot \nabla) \mathbf{u} & =-\nabla p+\varrho \nu \nabla^{2} \mathbf{u}+\mathbf{F} \\
\partial_{t} T+(\mathbf{u} \cdot \nabla) T & =\kappa \nabla^{2} T \\
\nabla \cdot \mathbf{u} & =0
\end{aligned}
$$

Die Navier-Stokes-Gleichung (2.1a) beschreibt die zeitliche Bewegung eines Fluidteilchens unter dem Einfluss von Advektion, Druck und Diffusion. Es ist $\mathbf{u}$ die Geschwindigkeit, $p$ der Druck, $\varrho$ die Dichte und $\nu$ die kinematische Viskosität. Die zusätzliche äußere Kraft $\mathbf{F}$ beinhaltet im vorliegenden Fall die Schwerkraft und die Corioliskraft:

$$
\mathbf{F}=\varrho \mathbf{g}+2 \varrho(\mathbf{u} \times \boldsymbol{\Omega})=\varrho(g \cdot \hat{\mathbf{z}}+2 \Omega(\mathbf{u} \times \hat{\mathbf{z}}))
$$

Hierbei ist $\hat{\mathbf{z}}$ der Einheitsvektor in $z$-Richtung, die Schwerkraft wirkt nur in negative $z$-Richtung und $g$ ist der Betrag der Schwerebeschleunigung. Die Rotationsachse (Richtung der Winkelge- 
schwindigkeit $\Omega$ ) wird der Einfachheit halber so gewählt, dass sie in positive $z$-Richtung zeigt. Sie hat dem Betrage nach die Größe $\Omega$.

Die Wärmetransportgleichung (2.1b) beschreibt die zeitliche Entwicklung der Temperatur durch Advektion und Diffusion. Es ist $T$ die Temperatur, u wiederum die Geschwindigkeit und $\kappa$ die thermische Diffusivität. Schließlich gilt auch die Kontinuitätsgleichung (2.1c) für ein inkompressibles Fluid.

An dieser Stelle sollen kurz die Randbedingungen erwähnt werden. Für die Temperatur gilt:

$$
\left.T\right|_{(z=0)}=T_{1},\left.\quad T\right|_{(z=d)}=T_{2}
$$

Die Differenztemperatur zwischen den Platten ist somit $\Delta T=T_{1}-T_{2}$. Die horizontale Geschwindigkeit an den Platten ist, wie oben schon erwähnt, nicht vorgegeben. Lediglich die Ableitung muss verschwinden. Die z-Komponente der Geschwindigkeit muss jedoch für eine feste, undurchlässige Wand verschwinden:

$$
\begin{gathered}
\left.\partial_{z} u_{x}\right|_{(z=0)}=0=\left.\partial_{z} u_{x}\right|_{(z=d)} \\
\left.\partial_{z} u_{y}\right|_{(z=0)}=0=\left.\partial_{z} u_{y}\right|_{(z=d)} \\
\left.u_{z}\right|_{(z=0)}=0=\left.u_{z}\right|_{(z=d)}
\end{gathered}
$$

Auf die Navier-Stokes-Gleichung wendet man nun die Boussinesq-Näherung an (siehe z.B. [7; 31]). Dafür nimmt man an, dass das Fluid inkompressibel ist (die Dichte nicht mehr vom Druck abhängig ist) und sich die Dichte nur linear mit der Temperatur ändert, also:

$$
\varrho-\varrho_{0}=\varrho_{0} \alpha\left(T-T_{0}\right)
$$

Hierbei sind $T_{0}$ und $\varrho_{0}$ eine mittlere Temperatur bzw. Dichte. Der thermische Ausdehungskoeffizient $\alpha$ soll klein sein, so dass die Dichteänderungen bei kleinen Temperaturänderungen ebenfalls klein sind. Damit können sie in allen Termen bis auf den Auftriebsterm vernachlässigt werden:

$$
\partial_{t} \mathbf{u}+(\mathbf{u} \cdot \nabla) \mathbf{u}+2 \Omega(\hat{\mathbf{z}} \times \mathbf{u})=-\frac{1}{\varrho_{0}} \nabla \pi+\nu \nabla^{2} \mathbf{u}+g \alpha \theta \hat{\mathbf{z}}
$$

Hierbei wurden außerdem die absoluten Größen des Drucks $p$ und der Temperatur $T$ ausgedrückt durch ihre Änderungen $\pi$ und $\theta$ von einem statischen Profil, also $\theta=T-T_{s}$ und $\pi=p-p_{s}$. Das statische Temperaturprofil $T_{s}$ bildet sich in dem Fall, dass keine Geschwindigkeit herrscht, nur durch thermische Diffusion aus. Es ist somit linear und gehorcht der Form:

$$
T_{s}=T_{1}-\Delta T \frac{z}{d}
$$




\subsubsection{Entdimensionalisierte Gleichungen}

Nun werden die Gleichungen entdimensionalisiert. Alle physikalischen Größen wie Länge, Zeit, Geschwindigkeit, Temperatur, etc. werden dimensionslos gemacht, indem man Referenzgrößen einführt. In diesem Fall soll die Referenzlänge gerade die Zellhöhe $d$ sein, die Referenzzeit ist die thermische Diffusionszeit $d^{2} / \kappa$ und die Referenztemperatur ist gerade der Temperaturunterschied $\Delta T$. Alle weiteren dimensionslosen Größen ergeben sich durch eine Kombination aus diesen:

$$
\begin{array}{rlrl}
\mathbf{x}^{\prime} & =\mathbf{x} \cdot \frac{1}{d} \\
t^{\prime} & =t \cdot \frac{\kappa}{d^{2}} & \Longrightarrow \quad \mathbf{u}^{\prime} & =\mathbf{u} \cdot \frac{d}{\kappa} \\
\theta^{\prime} & =\theta \cdot \frac{1}{\Delta T} & \mathbf{\Omega}^{\prime} & =\Omega \cdot \frac{d^{2}}{\nu} \\
\pi^{\prime} & =\pi \cdot \frac{d^{2}}{\varrho_{0} \kappa^{2}}
\end{array}
$$

Natürlich könnte man für die Referenzgrößen auch eine andere Wahl treffen, zum Beispiel für die Referenzlänge die Breite des Simulationsgebiets, aber die obige Entdimensionalisierung ist die übliche.

Aus den Gleichungen (2.1a-2.1c) ergeben sich damit:

$$
\begin{aligned}
\partial_{t}^{\prime} \mathbf{u}^{\prime}+\left(\mathbf{u}^{\prime} \cdot \nabla^{\prime}\right) \mathbf{u}^{\prime}+2 \operatorname{Pr} E k^{-1}\left(\hat{\mathbf{z}} \times \mathbf{u}^{\prime}\right) & =-\nabla^{\prime} \pi^{\prime}+\operatorname{Pr} \nabla^{\prime 2} \mathbf{u}^{\prime}+\operatorname{RaPr} \theta^{\prime} \hat{\mathbf{z}} \\
\partial_{t}^{\prime} \theta^{\prime}+\left(\mathbf{u}^{\prime} \cdot \nabla^{\prime}\right) \theta^{\prime} & =\nabla^{\prime 2} \theta^{\prime}+\mathbf{u}^{\prime} \cdot \hat{\mathbf{z}} \\
\nabla^{\prime} \cdot \mathbf{u}^{\prime} & =0
\end{aligned}
$$

Der Einfachheit halber werden im Folgenden wieder die Striche an den Variablen weggelassen. Alle folgenden Gleichungen sind also dimensionslos.

Die Charakterisierung des Fluids, das normalerweise durch Materialkonstanten wie z.B. Dichte $\varrho$, Viskosität $\nu$ oder die Temperaturleitfähigkeit $\kappa$ bestimmt wurde, geschieht nun durch Kennzahlen. Diese sind dimensionslos und beinhalten alle Materialkonstanten sowie die Parameter des Systems, die für das Problem wichtig sind.

\subsection{2 Überblick über die Kennzahlen}

Die Kennzahlen, die das System beschreiben, sind erstens die Rayleigh-Zahl:

$$
R a=\frac{g \alpha d^{3} \Delta T}{\nu \kappa}
$$


Sie beschreibt das Verhältnis von Auftriebskräften und viskosen Kräften. Konvektion setzt erst ab einer kritischen Rayleigh-Zahl $R a_{c}$ ein, die im vorliegenden Problem abhängig ist von der Ekman-Zahl Ek (siehe dazu Abschnitt 2.3). Diese ist definiert als

$$
E k=\frac{\nu}{\Omega d^{2}} \quad \text { mit } \quad \Omega=|\Omega|
$$

Sie gibt das Verhältnis zwischen viskosen Kräften und der Corioliskraft an. Bei der gewählten Entdimensionalisierung ist sie einfach das Reziproke der dimensionslosen Rotationsrate. Eine analoge, ebenfalls häufig in der Literatur anzutreffende Kennzahl ist die Taylorzahl, definiert als $T a=4 E k^{-2}$. Die Prandtl-Zahl schließlich ist:

$$
\operatorname{Pr}=\frac{\nu}{\kappa}
$$

Sie ist eine Kennzahl, die nur vom Fluid abhängt, und ist definiert als Verhältnis zwischen kinematischer Viskosität und Temperaturleitfähigkeit.

Neben diesen drei Kennzahlen, die das System beschreiben und zur Lösung des Gleichungssystems natürlich bekannt sein müssen, gibt es noch weitere Kennzahlen, die Kräfteverhältnisse innerhalb des Fluids angeben. Die wichtigsten für die Konvektion mit Rotation sind Nusseltund Reynolds-Zahl. Daraus können die Péclet- und Rossby-Zahl abgeleitet werden.

Die Nusselt-Zahl ist ein Maß für den Wärmetransport eines konvektierenden Fluids im Vergleich zum diffusiven Wärmetransport. Vor dem Einsetzen der Konvektion wird Wärme allein durch den instabilen Temperaturgradienten transportiert, d.h. sie diffundiert. Übersteigt die Rayleigh-Zahl eine kritische Grenze, so wird zusätzlich Wärme durch Konvektion, also durch die Bewegung des Fluids, transportiert. Die Nusselt-Zahl ist definiert als:

$$
N u=\frac{Q D}{l \Delta T}
$$

Es ist $Q$ der charakteristische Wärmefluss, $D$ eine charakteristische Länge und $l$ die thermische Leitfähigkeit. Die Nusselt-Zahl lässt sich aber auch über die Geschwindigkeit und die Temperatur ausdrücken:

$$
N u(z)=\left\langle u_{z} \cdot T\right\rangle_{x, y}-\partial_{z}\langle T\rangle_{x, y}
$$

Dies ergibt sich aus der Wärmetransportgleichung (2.1b), die in dimensionsloser Schreibweise diese Form hat:

$$
\partial_{t} T+(\mathbf{u} \cdot \nabla) T=\Delta T
$$

Nun mittelt man diesen Ausdruck über die horizontalen Richtungen:

$$
\partial_{t}\langle T\rangle_{x, y}=-\partial_{z}\left(\left\langle u_{z} \cdot T\right\rangle_{x, y}-\partial_{z}\langle T\rangle_{x, y}\right)
$$


In Gleichung (2.6) ist der Ausdruck $\left\langle u_{z} \cdot T\right\rangle_{x, y}-\partial_{z}\langle T\rangle_{x, y}$ gerade die vertikale Wärmestromdichte. Diese ist für den Fall ohne Konvektion gerade eins (die Geschwindigkeit ist $u_{z}=0$ und die Ableitung des statischen Temperaturprofils ist $\partial_{z} T=-1$ ). Damit ergibt sich der einfache Ausdruck in Gleichung (2.5) für die Nusselt-Zahl. In turbulenten Strömungen ist die Nusselt-Zahl eine zeitabhängige Größe. Deshalb wird sie als zeitliches Mittel aus den Simulationsergebnissen berechnet. Dabei ist darauf zu achten, dass über einen genügend langen Zeitraum gemittelt wird (siehe dazu auch Abschnitt 2.2.3). Eine so berechnete Nusselt-Zahl ist auch nicht mehr von der $z$-Koordinate abhängig, da keine Wärme durch die Seitenwände verloren gehen kann. Es ist also mit Gleichung (2.5)

$$
N u=\langle N u(z)\rangle_{t}
$$

Die Reynolds-Zahl ist eine Kennzahl, die das Verhältnis von Trägheitskräften zu viskosen Kräften beschreibt. Sie ist definiert als

$$
R e=\frac{U D}{\nu}
$$

Hierbei ist $U$ eine typische Geschwindigkeit und $D$ eine typische Länge des Systems. Eine typische Geschwindigkeit ist z.B. die mittlere (dimensionslose) Geschwindigkeit $U^{\prime}$, die auch zur Definition der kinetischen Energie in der Strömung verwendet wird:

$$
U^{\prime}=\sqrt{2\left\langle E_{k i n}\right\rangle_{t}}=U \frac{d}{\kappa} \quad \text { mit } \quad E_{k i n}=\frac{1}{2}\left\langle u_{x}^{2}+u_{y}^{2}+u_{z}^{2}\right\rangle_{V}
$$

Die typische Länge wird mit der (dimensionslosen) Zellhöhe $D^{\prime}=D \frac{1}{d}=1$ identifiziert. Also ist

$$
R e=\frac{\kappa d}{d} \frac{U^{\prime} \cdot D^{\prime}}{\nu}=\frac{1}{\operatorname{Pr}} U^{\prime}
$$

Das Produkt aus Prandtl- und Reynolds-Zahl wird Péclet-Zahl genannt. Sie beschreibt das Verhältnis von konvektiv transportierter zu geleiteter Wärme:

$$
P e=\frac{U D}{\kappa}=\operatorname{Re} \operatorname{Pr}
$$

Die Rossby-Zahl schließlich gibt das Verhältnis zwischen den Trägheitskräften und der Corioliskraft an. Sie ist definiert als:

$$
R o=\frac{U}{\Omega D}=R e E k
$$


Die in der Einleitung angesprochene Rotations-Péclet- bzw. Rotations-Reynolds-Zahl wird zusammen mit den Ergebnissen in Kapitel 5.3.2 bzw. 5.4.1 hergeleitet. Zusätzlich werden in Kapitel 5.3.3 für den Übergangsbereich zwischen rotationsdominiertem und rotationsfreiem Regime noch weitere, diffusionsfreie Kennzahlen definiert werden.

In Tabelle 2.1 sind die geschätzten Kennzahlen für den Erdkern aufgelistet (aus [5]). Daneben sind auch die Kennzahlen bzw. der Kennzahlbereich der Simulationen dieser Arbeit eingetragen.

\begin{tabular}{|c|c|c|c|}
\hline Kennzahl & Definition & Erdkern & Simulation \\
\hline Rayleigh & $\frac{\alpha g \Delta T d^{3}}{\nu \kappa}$ & $\sim 10^{25}-10^{35}$ & $10^{3}-10^{8}$ \\
Prandtl & $\frac{\nu}{\kappa}$ & $\sim 0.01-0.1$ & 0.7 und 7.0 \\
Ekman & $\frac{\nu}{\Omega d^{2}}$ & $\sim 10^{-15}$ & $2 \cdot 10^{-5}-\infty$ \\
Nusselt & $\frac{Q d}{l \Delta T}$ & $1-30$ & $1-60$ \\
Reynolds & $\frac{u d}{\nu}$ & $\sim 10^{9}$ & $10^{-1}-10^{3}$ \\
Rossby & $\frac{u}{\Omega d}$ & $\sim 10^{-6}$ & $10^{-3}-10^{1}$ \\
\hline
\end{tabular}

Tab. 2.1: Gegnüberstellung der Kennzahlen des Erdkerns (aus [5]) und der Simulationen dieser Arbeit.

Die ersten drei Zeilen sind die Parameter der Simulation, die letzten drei Zeilen die ErgebnisKennzahlen. Es ist erkennbar, dass die Kennzahlen der Simulation bei weitem nicht an die herankommen, die man in der Realität findet. Es ist allerdings auch nicht unbedingt nötig, die Werte der Realität zu simulieren, wenn die Kennzahlen des untersuchten Systems ein asymptotisches Verhalten aufweisen. Wenn man ein solches Verhalten im Modell findet, dann ist es durchaus gerechtfertigt, das Modell zu den Werten der Realität hin zu extrapolieren.

\subsubsection{Zeitskalen}

Es stellt sich die Frage, wie lange man eine Simulation laufen lassen muss, um ein verlässliches Ergebnis z.B. für die Nusselt-Zahl zu erhalten. Für laminare Strömungen sicherlich so lange, bis sich ein konstanter Wert eingestellt hat. Für turbulente Strömungen wird man einen solchen Endzustand nicht mehr erreichen. In diesem Fall ist die Simulation ,auskonvergiert“, wenn sich der zeitliche Mittelwert nur noch wenig ändert. Eine andere Möglichkeit wäre die Betrachtung des laufenden zeitlichen Mittels.

Quantitativ kann man sich an den typischen Zeitskalen des Systems orientieren. Eine konvektive Zeitskala $t_{c}$ wird definiert über die Zeit, die ein typisches Fluidteilchen benötigt, um einen Umlauf einer Konvektionsrolle mitzumachen. Für dieses typische Fluidteilchen setzt sich der 
mittlere Weg zusammen aus den horizontalen und vertikalen Abmessungen einer Konvektionsrolle (siehe auch Abbildung 2.1). In horizontaler Richtung muss das Fluidteilchen zwei mal den Weg $\lambda / 2$ und in vertikaler Richtung zweimal den Weg (der Zellhöhe) $d$ zurücklegen. Damit ergibt sich, im entdimensionalisierten System, als Gesamtweg $\lambda+2$. Die mittlere Geschwindigkeit wird über die kinetische Energie nach Gleichung (2.7) definiert:

$$
\left.\begin{array}{lll}
\text { mittlerer Weg } & =\lambda+2 \\
\text { mittlere Geschwindigkeit } & =\sqrt{2\left\langle E_{k i n}\right\rangle_{t}}
\end{array}\right\} \Longrightarrow t_{c}=\frac{\lambda+2}{\sqrt{2\left\langle E_{k i n}\right\rangle_{t}}}
$$

Die Simulationen wurden immer mindestens 100 konvektive Zeitskalen lang laufen gelassen, um auskonvergierte Ergebnisse zu liefern.

\subsection{Lineare Stabilitätsanalyse für verschiedene Ekman- Zahlen}

Wie schon vorher erwähnt, hängt die kritische Rayleigh-Zahl $R a_{c}$ von der Ekman-Zahl ab. Die kritische Rayleigh-Zahl erhält man durch eine lineare Stabilitätsanalyse des Gleichungssystems. Das ist ausführlich in [10] behandelt.

Die kritische Rayleigh-Zahl für den Fall ohne Rotation ist abhängig von den verwendeten Randbedingungen. Für schubspannungsfreie Ränder oben und unten ist sie verständlicherweise kleiner als mit no-slip Rändern. Die fehlenden Grenzschichten (und damit die geringere Reibung) führen dazu, dass schon bei einer kleineren Temperaturdifferenz Konvektionsströmungen entstehen. In Tabelle 2.2 sind die kritischen Rayleigh-Zahlen verzeichnet.

\begin{tabular}{|c|l|}
\hline kritische Rayleigh-Zahl $R a_{c}$ & \multicolumn{1}{|c|}{ Randbedingungen } \\
\hline 657,5 & $\begin{array}{l}\text { schubspannungsfreie Ränder oben und unten } \\
\text { ein schubspannungsfreier und ein no-slip } \\
\text { Rand (z.B. im Erdmantel) } \\
1107 \\
1708\end{array}$ \\
& $\begin{array}{l}\text { no-slip Ränder oben und unten (z.B. im äu- } \\
\text { ßeren Erdkern) }\end{array}$ \\
\hline
\end{tabular}

Tab. 2.2: Vergleich der kritischen Rayleigh-Zahlen $R a_{c}$ für verschiedene Randbedingungen.

Für den Fall mit Rotation sind in Tabelle 2.3 die Literaturwerte (aus [10]) für verschiedene 
Ekman-Zahlen verzeichnet. Es ist anzumerken, dass diese (im vorliegenden Fall der rotierenden ebenen Schicht) unabhängig von der Prandtl-Zahl sind.

Im Programm ist es möglich, den nichtlinearen Advektionsterm abzuschalten und somit eine linearisierte Rechnung durchzuführen. Um die kritische Rayleigh-Zahl und die kritische Wellenlänge zu bestimmen, startet man Rechnungen mit genügend großem Aspektverhältnis und einem zufälligen Temperaturfeld. Dabei wählt man Rayleigh-Zahlen, die knapp ober- und knapp unterhalb der kritischen Rayleigh-Zahl liegen. Für $R a<R a_{c}$ beobachtet man eine exponentiell abfallende Lösung (z.B. bei der Betrachtung der kinetischen Energie) und für $R a>R a_{c}$ eine exponentiell ansteigende Lösung. Die jeweiligen Abkling- und Anwachsraten $\beta$ trägt man über der Rayleigh-Zahl auf und erhält die kritische Rayleigh-Zahl durch Interpolation dort, wo $\beta=0$ ist. Dies ist exemplarisch in Abbildung 2.2 gezeigt für zwei verschiedene Ekman-Zahlen.
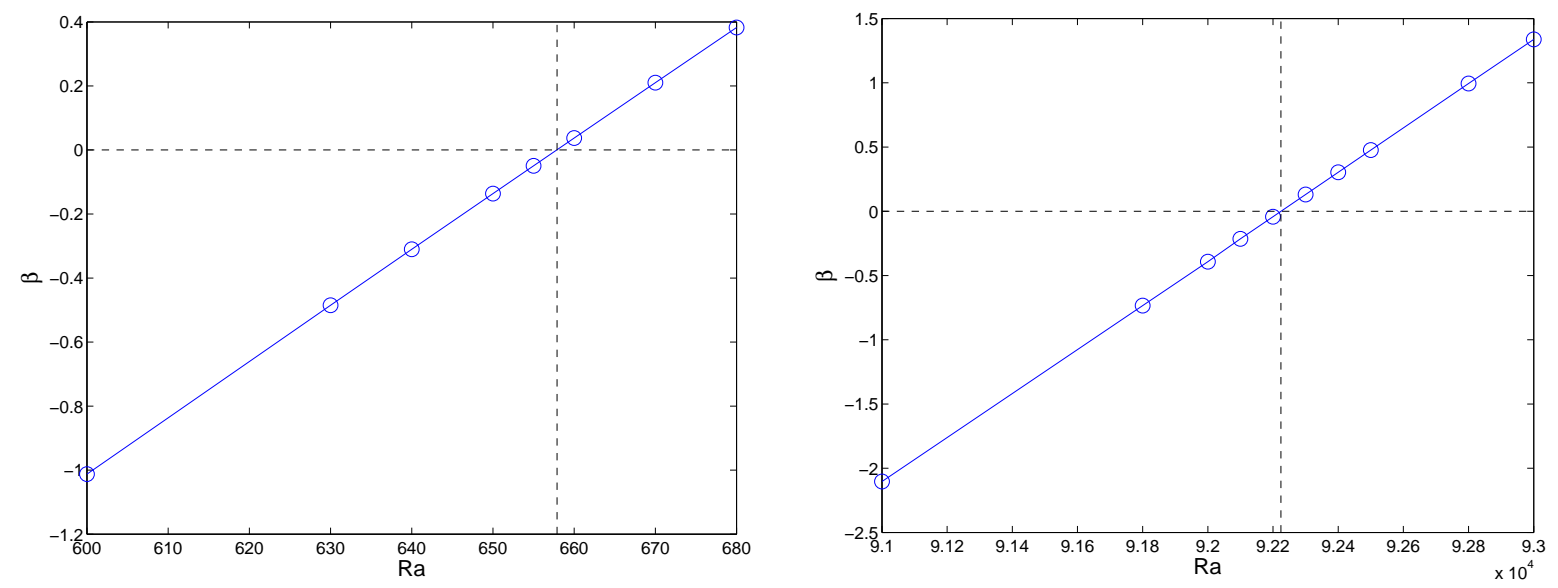

Abb. 2.2: Bestimmung des Onsets durch Linearisieren: Abkling- und Anwachsraten $\beta$ für $E k=\infty$ (links) und $E k=2 \cdot 10^{-3}$ (rechts).

Die kritischen Wellenlängen sollten sich, wenn das Aspektverhältnis nur groß genug ist, von alleine einstellen. Wie man in Tabelle 2.3 sieht, ist das auch der Fall. Trägt man die kritischen Rayleigh-Zahlen $R a_{c}$ und die kritischen Wellenlängen $\lambda_{c}$ aus der Literatur und der Simulation über der Ekman-Zahl auf, so erhält man Abbildung 2.3.

Man erkennt eine gute Übereinstimmung der Simulation mit der Theorie. Für genügend kleine Ekman-Zahlen $\left(E k \leq 1 \cdot 10^{-2}\right)$ gibt es ein Potenzgesetz für die Abhängigkeit zwischen der kritischen Rayleigh-Zahl $R a_{c}$ und der Ekman-Zahl Ek:

$$
R a_{c} \propto a_{R a} E k^{-4 / 3}
$$

Analog erhält man für die kritische Wellenlänge $\lambda_{c}$ :

$$
\lambda_{c} \propto a_{\lambda} E k^{1 / 3}
$$



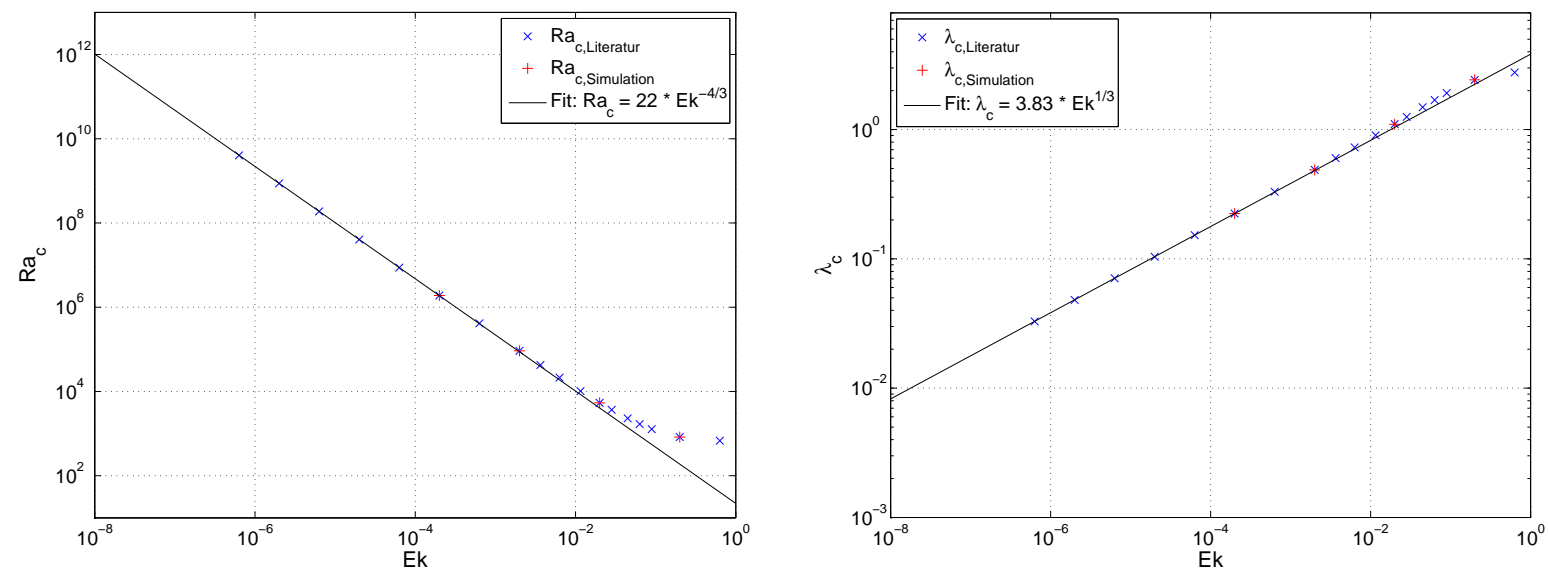

Abb. 2.3: Vergleich zwischen Literatur (aus [10]) und linearisierten Simulationen. Ein linearer Fit für genügend kleine Ekman-Zahlen ist ebenfalls eingetragen.

Dieses Potenzgesetz gilt für jegliche Randbedingungen, nur die Vorfaktoren unterscheiden sich. Für den vorliegenden Fall mit schubspannungsfreien Rändern oben und unten sind sie $a_{R a}=22$ und $a_{\lambda}=3.83$.

\begin{tabular}{|c|c|c|c|c|c|}
\hline & & \multicolumn{2}{|c|}{ Literatur } & \multicolumn{2}{c|}{ eigene Simulation } \\
$T a$ & $E k$ & $R a_{c}$ & $\lambda_{c}$ & $R a_{c}$ & $\lambda_{c}$ \\
\hline 0 & $\infty$ & $6.575 \cdot 10^{2}$ & 2.8138 & $6.5754 \cdot 10^{2}$ & 2.8282 \\
$1 \cdot 10^{2}$ & $2.000 \cdot 10^{-1}$ & $8.263 \cdot 10^{2}$ & 2.4222 & $8.2639 \cdot 10^{2}$ & 2.4253 \\
$1 \cdot 10^{4}$ & $2.000 \cdot 10^{-2}$ & $5.377 \cdot 10^{3}$ & 1.1027 & $5.3774 \cdot 10^{3}$ & 1.0988 \\
$1 \cdot 10^{6}$ & $2.000 \cdot 10^{-3}$ & $9.222 \cdot 10^{4}$ & 0.48858 & $9.2228 \cdot 10^{4}$ & 0.4877 \\
$1 \cdot 10^{8}$ & $2.000 \cdot 10^{-4}$ & $1.897 \cdot 10^{6}$ & 0.22424 & $1.8972 \cdot 10^{6}$ & 0.22387 \\
\hline
\end{tabular}

Tab. 2.3: Vergleich der kritischen Rayleigh-Zahl $R a_{c}$ bzw. der kritischen Wellenlänge $\lambda_{c}$ für verschiedene Ekman-Zahlen. Die Literaturwerte sind aus [10] entnommen. 


\section{Kapitel 3}

\section{Numerische Umsetzung}

In diesem Kapitel wird die Behandlung der Gleichungen besprochen, wie sie im vorigen Kapitel hergeleitet wurden. Dazu gehört zum einen das Aufstellen der endgültigen Gleichungen aus den Gleichungen (2.4a-2.4c) und zum anderen die räumliche und zeitliche Diskretisierung dieser.

Zum Ende des Kapitels wird noch an einem Beispiel die Aufstellung des Gleichungssystems bzw. der damit verbundenen Matrizen vorgeführt.

\subsection{Behandlung der Navier-Stokes-Gleichung und der Temperaturgleichung}

\subsubsection{Behandlung der Navier-Stokes-Gleichung: Aufspaltung der Ge- schwindigkeit}

Das Vektorfeld der Geschwindigkeit ist solenoidal, da aufgrund der Inkompressibilität des Fluids keine Quellen oder Senken im Geschwindigkeitsfeld vorhanden sind. Deshalb kann man es aufspalten in einen Toroidalanteil $\Psi$, einen Poloidalanteil $\Phi$ und einen Meanflow U (siehe z.B. [34]):

$$
\mathbf{u}(x, y, z, t)=\nabla \times \nabla \times(\Phi(x, y, z, t) \hat{\mathbf{z}})+\nabla \times(\Psi(x, y, z, t) \hat{\mathbf{z}})+\mathbf{U}(z, t)
$$

Im Gegensatz zum vektorwertigen Geschwindigkeitsfeld $\mathbf{u}$ sind das Toroidal- und das Poloidalfeld skalar. Sie sind außerdem periodisch und mittelwertfrei; damit ist gewährleistet, dass sie mittels einer FFT in den Fourierraum transformiert werden können. U enthält schließlich den Mittelwert der Geschwindigkeit in $x$ - und $y$-Richtung, Meanflow genannt, der aufgrund der 
gewünschten Periodizität von $\Phi(x, y, z, t)$ und $\Psi(x, y, z, t)$ separat behandelt werden muss. Die Kontinuitätsgleichung wird durch die Zerlegung automatisch erfüllt.

Die Bewegungsgleichung (2.4a) für die Geschwindigkeit muss nun in den neuen Größen $\Phi, \Psi$ und U ausgedrückt werden. Dies geschieht, indem man die Rotation von Gleichung (2.4a) bildet. Die z-Komponente davon ist die gesuchte Gleichung für das Toroidalfeld:

$$
-\left(\partial_{x}^{2}+\partial_{y}^{2}\right)\left[\left(\partial_{t}-\operatorname{Pr} \nabla^{2}\right) \Psi-2 \operatorname{Pr} E k^{-1} \partial_{z} \Phi\right]=\mathcal{R}_{\Psi}
$$

Für das Poloidalfeld bildet man entsprechend zweimal die Rotation und nimmt die z-Komponente:

$$
\left(\partial_{x}^{2}+\partial_{y}^{2}\right)\left[\nabla^{2}\left(\partial_{t}-\operatorname{Pr} \nabla^{2}\right) \Phi-2 \operatorname{Pr} E k^{-1} \partial_{z} \Psi\right]=\mathcal{R}_{\Phi}
$$

Die jeweiligen rechten Seiten sind:

$$
\begin{aligned}
& \mathcal{R}_{\Psi}=-\hat{\mathbf{z}} \cdot[\nabla \times[(\nabla \times \mathbf{u}) \times \mathbf{u}]] \\
& \mathcal{R}_{\Phi}=-\left(\partial_{x}^{2}+\partial_{y}^{2}\right) \operatorname{Ra} \operatorname{Pr} \Theta-\hat{\mathbf{z}} \cdot[\nabla \times \nabla \times[(\nabla \times \mathbf{u}) \times \mathbf{u}]]
\end{aligned}
$$

Die Gleichung für den Meanflow erhält man, indem man Gleichung (2.4a) über $x$ und $y$ mittelt:

$$
\begin{gathered}
\left(\partial_{t}-\operatorname{Pr} \partial_{z}^{2}\right)\left(\begin{array}{c}
U_{x} \\
U_{y}
\end{array}\right)+2 \operatorname{Pr} E k^{-1}\left(\begin{array}{c}
-U_{y} \\
U_{x}
\end{array}\right)=\mathcal{R}_{\mathbf{U}} \\
\mathcal{R}_{\mathbf{U}}=-\langle(\nabla \times \mathbf{u}) \times \mathbf{u}\rangle_{x, y}
\end{gathered}
$$

Der Meanflow hat natürlich keine $z$-Komponente, da das Fluid als inkompressibel angenommen wird und außerdem undurchlässige Wände oben und unten gesetzt sind.

Wie man sieht, taucht der Druck in den Gleichungen nicht mehr auf. Der Gradient des Drucks, der in Gleichung (2.4a) steht, fällt beim Bilden der Rotation bzw. des Mittelwertes weg. Ebenso fällt auf, dass die Corioliskraft, im Gegensatz zum Auftriebsterm, nicht auf den rechten Seiten der Toroidal- bzw. Poloidalgleichung steht. Der Grund dafür ist, dass so eine implizite Behandlung des Coriolisterms möglich ist. Dies wird in Abschnitt 3.3 ausführlich behandelt.

Möchte man umgekehrt die Geschwindigkeit u aus der Zerlegung berechnen, so gilt:

$$
\mathbf{u}=\left(\begin{array}{c}
\partial_{y} \Psi+\partial_{x} \partial_{z} \Phi+U_{x} \\
-\partial_{x} \Psi+\partial_{y} \partial_{z} \Phi+U_{y} \\
-\left(\partial_{x}^{2}+\partial_{y}^{2}\right) \Phi
\end{array}\right)
$$


Die Randbedingungen für die neuen Variablen $\Phi, \Psi$ und $\mathbf{U}$ können nun aus den geforderten Randbedingungen für die Geschwindigkeit $\mathbf{u}$ und Gleichung (3.8) berechnet werden. Diese sind im spannungsfreien Fall:

$$
\begin{aligned}
\left.\partial_{z} u_{x}\right|_{(z=0)} & =0=\left.\partial_{z} u_{x}\right|_{(z=1)} \\
\left.\partial_{z} u_{y}\right|_{(z=0)} & =0=\left.\partial_{z} u_{y}\right|_{(z=1)} \\
\left.u_{z}\right|_{(z=0)} & =0=\left.u_{z}\right|_{(z=1)}
\end{aligned}
$$

Das bedingt folgende Randbedingungen für $\Phi, \Psi$ und $\mathbf{U}$ :

$$
\begin{aligned}
\left.\Phi\right|_{(z=0)} & =0=\left.\Phi\right|_{(z=1)} \\
\left.\partial_{z}^{2} \Phi\right|_{(z=0)} & =0=\left.\partial_{z}^{2} \Phi\right|_{(z=1)} \\
\left.\partial_{z} \Psi\right|_{(z=0)} & =0=\left.\partial_{z} \Psi\right|_{(z=1)} \\
\left.\partial_{z} U_{x}\right|_{(z=0)} & =0=\left.\partial_{z} U_{x}\right|_{(z=1)} \\
\left.\partial_{z} U_{y}\right|_{(z=0)} & =0=\left.\partial_{z} U_{y}\right|_{(z=1)}
\end{aligned}
$$

Diese Randbedingungen werden später in die Zeitschritt-Matrix implementiert werden.

\subsubsection{Behandlung der Temperaturgleichung}

Die Temperaturgleichung (2.4b) ändert sich kaum. Sie lässt sich analog zu den Geschwindigkeitsgleichungen schreiben als:

$$
\left(\partial_{t}-\nabla^{2}\right) \theta=\mathcal{R}_{\theta}
$$

Die rechte Seite ist:

$$
\mathcal{R}_{\theta}=-\left(\partial_{x}^{2}+\partial_{y}^{2}\right) \Phi-(\nabla \cdot(\mathbf{u} \theta))
$$

Hierbei ist der linke Term der rechten Seite die Entsprechung für $\mathbf{u} \cdot \hat{\mathbf{z}}=u_{z}$ in den zerlegten Feldern, siehe Gleichung (3.8). Der rechte Term ist vereinfacht unter Benutzung der Kontinuitätsgleichung $(2.4 \mathrm{c})$.

Die Randbedingungen sind:

$$
\left.\theta\right|_{(z=0)}=0=\left.\theta\right|_{(z=1)}
$$




\subsection{Räumliche und zeitliche Diskretisierung}

\subsubsection{Zerlegung nach Fourier-Koeffizienten}

Wie schon angedeutet, sollen die Gleichungen in $x$ - und $y$-Richtung nach ihren Fourierkoeffizienten entwickelt werden. Dies wird für das Poloidal- und Toroidalfeld sowie für das Temperaturfeld gemacht. Für den Meanflow (3.6) ist es nicht nötig/möglich, da dieser nicht mehr von $x$ bzw. $y$ abhängt, sondern nur noch von $z$.

Die Felder im Ortsraum werden also ausgedrückt durch eine (endliche) Summe über die Fourierkoeffizienten im Spektralraum:

$$
\begin{aligned}
\Phi(\mathbf{x}, t) & =\sum_{\mathbf{k}} \hat{\Phi}_{\mathbf{k}}(z, t) e^{i \mathbf{k} \cdot \mathbf{r}} \\
\Psi(\mathbf{x}, t) & =\sum_{\mathbf{k}} \hat{\Psi}_{\mathbf{k}}(z, t) e^{i \mathbf{k} \cdot \mathbf{r}} \\
\theta(\mathbf{x}, t) & =\sum_{\mathbf{k}} \hat{\Theta}_{\mathbf{k}}(z, t) e^{i \mathbf{k} \cdot \mathbf{r}}
\end{aligned}
$$

Hierbei ist $\mathbf{r}=x \hat{\mathbf{x}}+y \hat{\mathbf{y}}$ der Ortsvektor in der $x y$-Ebene und $\mathbf{k}=k_{x} \hat{\mathbf{x}}+k_{y} \hat{\mathbf{y}}$ der zugehörige Wellenvektor der Fourierkoeffizienten. Diese hängen jetzt räumlich nur noch von $z$ und der Zeit $t$ ab. Die Wellenvektoren sind jeweils gegeben durch die Wellenzahlen $k_{x}$ und $k_{y}$, die von der gewählten Auflösung $N_{x}$ bzw. $N_{y}$ und dem Aspektverhältnis $\Gamma_{x}=\Gamma_{y}=\Gamma$ abhängen:

$$
k_{x, y}=\left(-\frac{N_{x, y}}{2}, \ldots-1,0,1, \ldots, \frac{N_{x, y}}{2}\right) \cdot\left(\frac{2 \pi}{\Gamma}\right)
$$

Man erkennt leicht, dass die gewählte Auflösung direkt bestimmt, welche Wellenzahlen/-längen noch aufgelöst werden können. Je größer die Auflösung ist, desto größere Wellenzahlen bzw. kleinere Wellenlängen können auch aufgelöst werden. Allerdings steigen damit natürlich auch der Speicherbedarf und die Rechenzeit an. Die Auflösung ist deshalb so zu wählen, dass die vorkommenden Strukturen im Strömungs- und Temperaturfeld ausreichend genau wiedergegeben werden. In den Tabellen der Simulationsergebnisse in Anhang A ist die verwendete Auflösung deshalb mit angegeben.

Um den Wechsel zwischen Orts- und Fourierraum schnell zu bewältigen, wird eine FFT aus [32] verwendet. Dazu ist es nötig, dass die Auflösung in beiden horizontalen Richtungen eine Potenz von zwei ist. Typisch sind Auflösungen von 128 oder 256 Punkten in $x$ - und $y$-Richtung. Setzt man die Entwicklungen (3.12a-3.12c) in die Gleichungen $(3.2,3.3,3.6,3.10)$ ein, so erhält 
man:

$$
\begin{aligned}
& \left(\partial_{z}^{2}-k^{2}\right)\left[\left(\partial_{t}-\operatorname{Pr}\left(\partial_{z}^{2}-k^{2}\right)\right) \hat{\Phi}_{\mathbf{k}}(z, t)\right]+2 \operatorname{Pr} E k^{-1} \partial_{z} \hat{\Psi}_{\mathbf{k}}(z, t)=\mathcal{R}_{\hat{\Phi}_{\mathbf{k}}}(z, t) \\
& \left(\partial_{t}-\operatorname{Pr}\left(\partial_{z}^{2}-k^{2}\right)\right) \hat{\Psi}_{\mathbf{k}}(z, t)-2 \operatorname{Pr} E k^{-1} \partial_{z} \hat{\Phi}_{\mathbf{k}}(z, t)=\mathcal{R}_{\hat{\Psi}_{\mathbf{k}}}(z, t) \\
& {\left[\partial_{t}-\left(\partial_{z}^{2}-k^{2}\right)\right] \hat{\Theta}_{\mathbf{k}}(z, t)=\mathcal{R}_{\hat{\Theta}_{\mathbf{k}}}(z, t)} \\
& {\left[\partial_{t}-\operatorname{Pr} \partial_{z}^{2}\right]\left(\begin{array}{c}
U_{x} \\
U_{y}
\end{array}\right)+2 \operatorname{Pr} E k^{-1}\left(\begin{array}{c}
-U_{y} \\
U_{x}
\end{array}\right)=\mathcal{R}_{\mathbf{U}}(z, t)}
\end{aligned}
$$

Die zugehörigen rechten Seiten sind:

$$
\begin{aligned}
& \mathcal{R}_{\hat{\Phi}_{\mathbf{k}}}(z, t)=-\operatorname{Ra} \operatorname{Pr} \hat{\Theta}_{\mathbf{k}}+\frac{1}{k^{2}}\{\hat{\mathbf{z}} \cdot[\nabla \times \nabla \times[(\nabla \times \mathbf{u}) \times \mathbf{u}]]\}_{\mathbf{k}} \\
& \mathcal{R}_{\hat{\Psi}_{\mathbf{k}}}(z, t)=-\frac{1}{k^{2}}\{\hat{\mathbf{z}} \cdot[\nabla \times[(\nabla \times \mathbf{u}) \times \mathbf{u}]]\}_{\mathbf{k}} \\
& \mathcal{R}_{\hat{\Theta}_{\mathbf{k}}}(z, t)=k^{2} \hat{\Phi}_{\mathbf{k}}-\{\nabla \cdot(\mathbf{u} \Theta)\}_{\mathbf{k}} \\
& \mathcal{R}_{\mathbf{U}}(z, t)=-\langle(\nabla \times \mathbf{u}) \times \mathbf{u}\rangle_{x, y}
\end{aligned}
$$

Hierbei meint die Schreibweise $\langle\ldots\rangle_{x, y}$ das Mittel über eine $x y$-Ebene und $\{\ldots\}_{\mathbf{k}}$ die entsprechende Fourierkomponente.

Die linken Seiten beinhalten nur Variablen im Fourierraum. Auf den Meanflow bzw. seine beiden Komponenten trifft das insofern auch zu, als dass der Vorfaktor in Gleichung (3.14d) der gleiche ist wie für das Toroidalfeld in Gleichung (3.14b) bei $k^{2}=0$. Die Gleichungen der rechten Seiten hingegen beinhalten die Produkte $(\nabla \times \mathbf{u}) \times \mathbf{u}$ und $(\mathbf{u} \Theta)$. Diese Produkte im Ortsraum entsprechen im Spektralraum einer Faltung. Da es einfacher ist, das Produkt im Ortsraum zu bilden, werden die Daten in den Ortsraum und das Ergebnis der Berechnungen in den Spektralraum zurücktransformiert. Dies ist ausführlich in Abschnitt 4.4 beschrieben.

\subsubsection{Zerlegung nach Chebyshev-Koeffizienten}

In den Gleichungen (3.14a-3.14d) sind die Werte der Fourierkoeffizienten $\hat{\Phi}(z, t), \hat{\Psi}(z, t), \hat{\Theta}(z, t)$ und $\mathbf{U}(z, t)$ räumlich nur noch von $z$ abhängig. Die Verwendung nicht-periodischer Randbedingungen in $z$-Richtung schlieft die Verwendung einer Fourierentwicklung aus. Im Ursprungsprogramm aus [19] werden Chebyshev-Polynome verwendet. Dies soll auch hier beibehalten werden.

Die Stützstellen $z_{i}$ in $z$-Richtung, an denen die Funktionen ausgewertet werden, sollen verteilt 
sein nach der Formel:

$$
z_{i}=\frac{1}{2} \cos \left(\frac{\pi i}{N_{z}-1}\right)+\frac{1}{2} \text { für } i=0,1, \ldots, N_{z}-1
$$

Diese Verteilung hat zwei Vorteile: Zum einen befinden sich mehr Stützstellen in der Nähe der oberen und unteren Gebietsgrenze, wie in Abbildung 3.1 zu sehen. Man erkennt, dass innerhalb
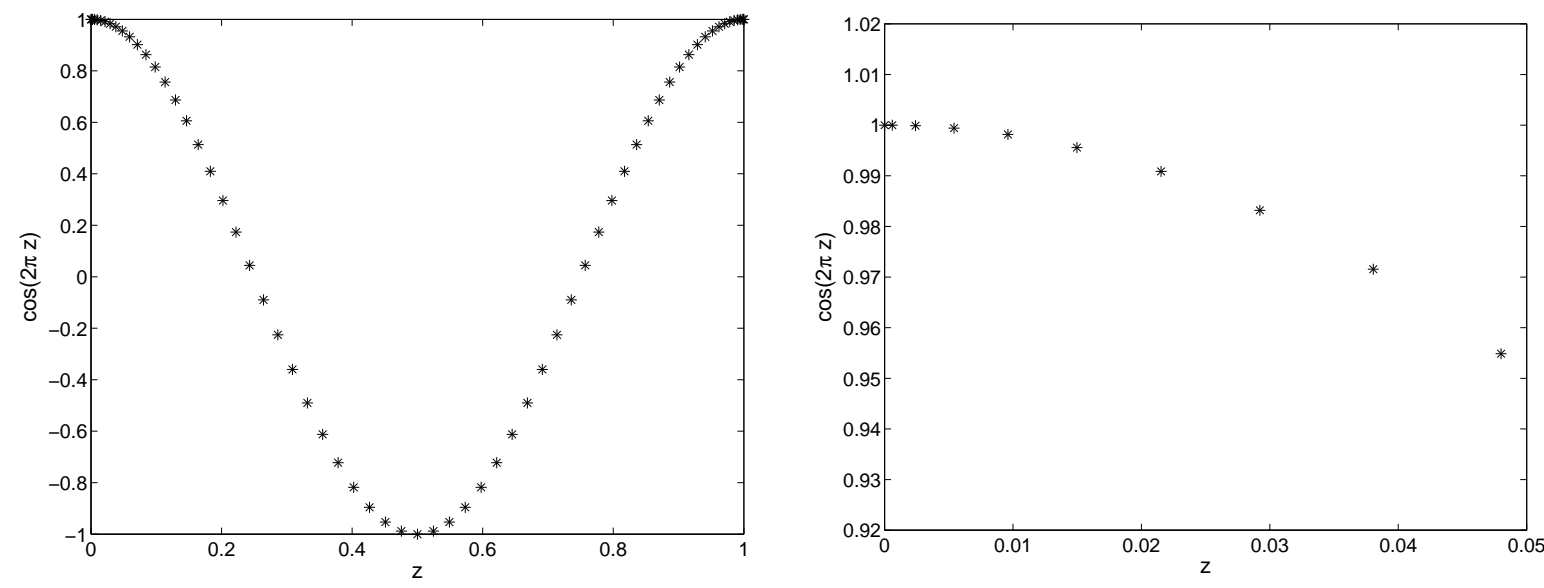

Abb. 3.1: Links: Verteilung der Stützstellen für $N_{z}=65$ Chebychev-Polynome. Rechts: Vergrößerung des Randbereichs.

der ersten 5 Prozent des Gebiets 10 Stützstellen liegen. Bei äquidistant verteilten Stützstellen wären zur Diskretisierung der gesamten $z$-Richtung 200 Stützstellen nötig, mit der Verteilung aus Gleichung (3.16) sind es lediglich 65.

Der zweite Vorteil liegt in der Transformation zwischen Orts- und Spektralraum: Formt man Gleichung (3.16) so um, dass $z$ über den Definitionsbereich der Chebychev-Polynome $(\tilde{z}=2 z-1)$ läuft und benutzt Gleichung (B.2), so entspricht das gerade einer CosinusTransformation:

$$
\begin{gathered}
\tilde{z}_{i}=2 z_{i}-1 \quad \Longrightarrow \quad \tilde{z}_{i}=\cos \left(\frac{\pi i}{N_{z}-1}\right) \\
\Longrightarrow T_{n}(\tilde{z})=\cos \left(\frac{n \pi i}{N_{z}-1}\right)
\end{gathered}
$$

Analog zur FFT gibt es auch eine schnelle Cosinustransformation COST der "SLATEC Common Mathematical Library" [8]. Dazu muss die Anzahl der Stützstellen allerdings gerade $N_{z}=2^{n}+1$ sein. Typische Werte sind $N_{z}=65$ oder $N_{z}=129$ :

Es gilt also für die Variablen zu einem konstanten Wellenvektor bzw. den Meanflow:

$$
\hat{\Phi}_{\mathbf{k}}(z, t)=\sum_{n=0}^{N_{z}-1} f_{\mathbf{k}, n}(t) T_{n}(\tilde{z})
$$


Analog für die übrigen Variablen. Die rechten Seiten sind beispielhaft:

$$
\mathcal{R}_{\hat{\Phi}_{\mathbf{k}}}(z, t)=\sum_{n=0}^{N_{z}-1} b_{\hat{\Phi}_{\mathbf{k}}, n}(t) T_{n}(\tilde{z})
$$

Die Randbedingungen sind durch diese Definitionen noch nicht festgelegt. Für das Toroidalund Temperaturfeld sowie die Meanflowkomponenten werden 2 und für das Poloidalfeld 4 Randbedingungen benötigt, siehe Gleichungen (3.9a-3.9e). Um diese Randbedingungen zu erfüllen, müssen die Chebyshev-Polynome beim Aufstellen der Zeitschritt-Matrix geeignet kombiniert werden (siehe das Beispiel in Abschnitt 3.3.1).

\subsubsection{Zeitliche Diskretisierung}

In diesem Abschnitt werden die zeitlichen Diskretisierungen, sowohl explizite als auch implizite, erläutert. Für weitere Informationen siehe [14].

Die Ergebnisse zu einem neuen Zeitpunkt $t^{n+1}=t^{n}+h$ mit der Zeitschrittweite $h$ werden aus den Werten der vorangegangenen Zeitschritte berechnet. Für die zeitliche Ableitung wird ein Euler-Schritt benutzt:

$$
\partial_{t} f_{i}(t) \quad \rightarrow \quad \frac{f_{i}^{n+1}-f_{i}^{n}}{h}
$$

Dies ist eine explizite Diskretisierung erster Ordnung. Die Zeitschrittweite $h$ muss dabei so gewählt werden, dass der Zeitschritt stabil bleibt. Dazu dient das CFL-Kriterium (nach Richard Courant, Kurt Friedrichs und Hans Lewy), das besagt, dass der gewählte Zeitschritt kleiner sein muss als der Quotient aus der Gitterweite $\Delta x$ und der maximalen Geschwindigkeit $u_{\max }=\sqrt{\mathbf{u} \cdot \mathbf{u}}$. Anschaulich gesprochen darf sich auch das schnellste Fluidpartikel innerhalb eines Zeitschrittes nicht weiter als bis zum nächsten Gitterpunkt bewegen:

$$
\Delta t_{C F L}=\frac{\Delta x}{u_{\max }}
$$

Die Zeitschrittweite muss nun kleiner sein als $\Delta t_{C F L}$, im Allgemeinen wurde $h=0.25 \Delta t_{C F L}$ gewählt.

Die restlichen Terme der linken Seite werden durch ein implizites Crank-Nicolson Verfahren diskretisiert:

$$
f_{i}(t) \quad \rightarrow \quad \frac{f_{i}^{n+1}+f_{i}^{n}}{2}
$$

Es hat eine Genauigkeit zweiter Ordnung und den Vorteil, dass es inkonditionell stabil ist, wie eine von Neumann Stabilitätsanalyse zeigt. Für große Zeitschrittweiten können aber Oszillationen oder sogar instabile Lösungen entstehen. Damit dies nicht geschieht, darf die erlaubte 
Zeitschrittweite theoretisch doppelt so groß wie beim expliziten Euler-Verfahren sein. In der Praxis sind aber - je nach Problemstellung - viel größere Zeitschritte möglich.

Nahe am Onset tritt ein spezieller Fall ein. Man erwartet nach Gleichung (3.21), dass gerade dort große Zeitschritte möglich sind, da die Geschwindigkeiten noch klein sind. Allerdings wird dort, besonders für kleine Ekman-Zahlen, Gleichung (2.4a) durch den Coriolisterm dominiert. Eine explizite Behandlung des Coriolisterms würde also eine viel restriktivere Schranke für die Zeitschrittweite fordern. Um dies zu umgehen wird auch der Coriolisterm implizit behandelt. Allerdings werden dadurch die beiden Gleichungen (3.14a,3.14b) gekoppelt, was einen erhöhten numerischen Aufwand bedeutet. Wie weiter unten gezeigt, muss ein doppelt so großes Gleichungssystem gelöst werden. Dies rentiert sich natürlich nur, wenn der Nutzen durch einen größeren Zeitschritt die Kosten des erhöhten numerischen Aufwands überwiegt.

Die rechten Seiten werden mit einem expliziten Adams-Bashforth Verfahren zweiter Ordnung diskretisiert:

$$
b_{i}(t) \rightarrow \frac{3 b_{i}^{n}+b_{i}^{n-1}}{2}
$$

Hierbei ist zu beachten, dass Werte zu zwei vorangegangenen Zeitschritten benötigt werden. Da das zu Beginn einer Simulation nicht der Fall ist, startet jede neue Simulation mit einem Euler-Schritt für die rechten Seiten.

\subsection{Beispiel: Gleichungssystem für Poloidal- und Toroidal- feld}

Das Vorgehen zur Diskretisierung und zum Aufstellen des Gleichungssystems soll nun beispielhaft vorgeführt werden. Für die explizite Behandlung des Coriolisterms genügt es, nur z.B. das Toroidalfeld vorzuführen. Für die implizite Behandlung des Coriolisterms müssen beide Gleichung betrachtet werden, da das Poloidal- und Toroidalfeld gekoppelt sind.

\subsubsection{Expliziter Coriolisterm}

Ausgangspunkt für das Beispiel ist die Gleichung (3.14b). Allerdings wird hier nur die Gleichung für eine Fourierkomponente mit festem $\mathbf{k}$ betrachtet (weshalb dieser Index im Folgenden weggelassen wird):

$$
\left(\partial_{t}-\operatorname{Pr}\left(\partial_{z}^{2}-k^{2}\right)\right) \hat{\Psi}(z, t)=\mathcal{R}_{\hat{\Psi}}(z, t)
$$


Setzt man die Diskretisierungen (3.20,3.22,3.23) und die Entwicklungen $(3.18,3.19)$ in Gleichung (3.24) ein, so erhält man:

$$
\begin{aligned}
\sum_{i=0}^{N_{z}-1} & {\left[\left(1+\frac{h}{2} \operatorname{Prk}^{2}-\frac{h}{2} \operatorname{Pr}_{z}^{2}\right) \hat{\psi}_{i}^{n+1}\right] T_{i}(\tilde{z}) } \\
& =\sum_{i=0}^{N_{z}-1}\left[\left(1-\frac{h}{2} \operatorname{Prk}^{2}+\frac{h}{2} \operatorname{Pr}_{z}^{2}\right) \hat{\psi}_{i}^{n}\right] T_{i}(\tilde{z}) \\
& +\sum_{i=0}^{N_{z}-1} \frac{h}{2}\left(3 b_{\hat{\psi}, i}^{n}+b_{\hat{\psi}, i}^{n-1}\right) T_{i}(\tilde{z})
\end{aligned}
$$

Man erkennt gut, dass in dem Gleichungssystem jeweils 3 Terme vorkommen: Erstens die noch unbekannten Werte zum gesuchten Zeitpunkt $n+1$, zweitens die bekannten Werte zum jetzigen Zeitpunkt $n$ und drittens die ebenfalls bekannten rechten Seiten zum jetzigen und vorigen Zeitpunkt.

Dieses Gleichungssystem lässt sich auch in Matrixform schreiben:

$$
\mathcal{M}_{\hat{\psi}}^{n+1} \cdot \hat{\boldsymbol{\psi}}^{n+1}=\mathcal{M}_{\hat{\psi}}^{n} \cdot \hat{\boldsymbol{\psi}}^{n}+\mathbf{b}_{\hat{\psi}}^{n}
$$

mit den Komponenten $b_{\hat{\psi}, i}^{n}=3 b_{\hat{\psi}, i}^{n}+b_{\hat{\psi}, i}^{n-1}$ der rechten Seite $\mathbf{b}_{\hat{\psi}}^{n}$.

Die quadratischen Matrizen haben den Rang $N_{z}$. Beim Aufstellen der Matrix müssen natürlich noch die Randbedingunen implementiert werden. Da es für das Toroidalfeld nach Gleichung (3.9c) zwei Randbedingungen gibt, nämlich verschwindende $z$-Ableitungen oben und unten, sind zwei Zeilen der Matrix dadurch gegeben. Mit Hilfe von Gleichung (B.5) können die Werte berechnet werden. Dadurch werden die untersten beiden Zeilen festgelegt. Die Matrix $\mathcal{M}_{\hat{\psi}}^{n+1}$ für $N_{z}=9$ sieht dann z.B. aus wie in Gleichung (3.25) (hierbei ist I die Einheitsmatrix). Zur Einhaltung der Randbedingung müssen die untersten beiden Werte der rechten Seite natürlich Null sein.

Zur Lösung der Gleichung muss die Matrix $\mathcal{M}_{\hat{\psi}}^{n+1}$ invertiert werden. Da sie konstant ist (unter der Voraussetzung, dass sich die Zeitschrittweite $h$ nicht ändert), können die Matrizen für alle Werte von $\mathbf{k}$ beim Start der Simulation LU-zerlegt und diese Zerlegung gespeichert werden. Damit hält sich der numerische Aufwand zum Lösen der Gleichung in Grenzen, da immer nur zum jeweiligen $k^{2}$ die entsprechende Zerlegung aus dem Speicher geholt und mit der rechten 
Seite gelöst werden muss.

$$
\mathcal{M}_{\hat{\psi}}^{n+1}=\mathbf{I}+\frac{h}{2} \operatorname{Pr}\left(\begin{array}{ccccccccc}
k^{2} & 0 & -16 & 0 & -128 & 0 & -432 & 0 & -1024 \\
0 & k^{2} & 0 & -96 & 0 & -480 & 0 & -1344 & 0 \\
0 & 0 & k^{2} & 0 & -192 & 0 & -768 & 0 & -1920 \\
0 & 0 & 0 & k^{2} & 0 & -320 & 0 & -1120 & 0 \\
0 & 0 & 0 & 0 & k^{2} & 0 & -480 & 0 & -1536 \\
0 & 0 & 0 & 0 & 0 & k^{2} & 0 & -672 & 0 \\
0 & 0 & 0 & 0 & 0 & 0 & k^{2} & 0 & -896 \\
0 & 1 & 4 & 9 & 16 & 25 & 36 & 49 & 64 \\
0 & 1 & -4 & 9 & -16 & 25 & -36 & 49 & -64
\end{array}\right)
$$

\subsubsection{Impliziter Coriolisterm}

Nun wird der Coriolisterm implizit behandelt. Da hierbei das Poloidal- und Toroidalfeld gekoppelt sind, müssen beide Gleichung betrachtet werden. Ausgangspunkt sind die Gleichungen $(3.14 \mathrm{a}, 3.14 \mathrm{~b})$, wieder wie eben für ein festes $\mathbf{k}$ :

$$
\begin{aligned}
\left(\partial_{z}^{2}-k^{2}\right)[ & \left.\left(\partial_{t}-\operatorname{Pr}\left(\partial_{z}^{2}-k^{2}\right)\right) \hat{\Phi}(z, t)\right]+2 \operatorname{Pr} E k^{-1} \partial_{z} \hat{\Psi}(z, t)=\mathcal{R}_{\hat{\Phi}} \\
\left(\partial_{t}-\operatorname{Pr}\left(\partial_{z}^{2}-k^{2}\right)\right) \hat{\Psi}(z, t)-2 \operatorname{Pr} E k^{-1} \partial_{z} \hat{\Phi}(z, t) & =\mathcal{R}_{\hat{\Psi}}
\end{aligned}
$$

Setzt man die Diskretisierungen $(3.20,3.22,3.23)$ und die Entwicklungen $(3.18,3.19)$ in die Gleichungen $(3.26,3.27)$ ein, so erhält man das Gleichungssystem für das Poloidalfeld:

$$
\begin{aligned}
\sum_{i=0}^{N_{z}-1} & {\left[\left(\partial_{z}^{2}-k^{2}\right)\left(1+\frac{h}{2} \operatorname{Pr} k^{2}-\frac{h}{2} \operatorname{Pr} \partial_{z}^{2}\right) \hat{\phi}_{i}^{n+1}-h \operatorname{Pr} E k^{-1} \partial_{z} \hat{\psi}_{i}^{n+1}\right] T_{i}(\tilde{z}) } \\
& =\sum_{i=0}^{N_{z}-1}\left[\left(\partial_{z}^{2}-k^{2}\right)\left(1-\frac{h}{2} \operatorname{Pr} k^{2}+\frac{h}{2} \operatorname{Pr}_{z}^{2}\right) \hat{\phi}_{i}^{n}+h \operatorname{Pr} E k^{-1} \partial_{z} \hat{\psi}_{i}^{n}\right] T_{i}(\tilde{z}) \\
& +\sum_{i=0}^{N_{z}-1} \frac{h}{2}\left(3 b_{\hat{\phi}, i}^{n}+b_{\hat{\phi}, i}^{n-1}\right) T_{i}(\tilde{z})
\end{aligned}
$$


Das Toroidalfeld sieht entsprechend aus:

$$
\begin{aligned}
\sum_{i=0}^{N_{z}-1} & {\left[\left(1+\frac{h}{2} \operatorname{Pr} k^{2}-\frac{h}{2} \operatorname{Pr}_{z}^{2}\right) \hat{\psi}_{i}^{n+1}-h \operatorname{Pr} E k^{-1} \partial_{z} \hat{\phi}_{i}^{n+1}\right] T_{i}(\tilde{z}) } \\
= & \sum_{i=0}^{N_{z}-1}\left[\left(1-\frac{h}{2} \operatorname{Prk}^{2}+\frac{h}{2} \operatorname{Pr} \partial_{z}^{2}\right) \hat{\psi}_{i}^{n}+h \operatorname{Pr} E k^{-1} \partial_{z} \hat{\phi}_{i}^{n}\right] T_{i}(\tilde{z}) \\
& +\sum_{i=0}^{N_{z}-1} \frac{h}{2}\left(3 b_{\hat{\psi}, i}^{n}+b_{\hat{\psi}, i}^{n-1}\right) T_{i}(\tilde{z})
\end{aligned}
$$

Diese beiden Gleichungssysteme lassen sich in einer Matrix zusammenfassen:

$$
\mathcal{M}^{n+1} \cdot \mathbf{x}^{n+1}=\mathcal{M}^{n} \cdot \mathbf{x}^{n}+\mathbf{b}^{n}
$$

Die Matrizen $\mathcal{M}^{n+1}$ und $\mathcal{M}^{n}$ sind so zusammengesetzt:

$$
\begin{aligned}
& \mathcal{M}^{n+1}=\left(\begin{array}{c|c}
\left(\partial_{z}^{2}-k^{2}\right)\left(1+\frac{h}{2} \operatorname{Pr} k^{2}-\frac{h}{2} \operatorname{Pr} \partial_{z}^{2}\right) & -h \operatorname{Pr} E k^{-1} \partial_{z} \\
\hline-h \operatorname{Pr} E k^{-1} \partial_{z} & 1+\frac{h}{2} \operatorname{Pr} k^{2}-\frac{h}{2} \operatorname{Pr} \partial_{z}^{2}
\end{array}\right) \\
& \mathcal{M}^{n}=\left(\begin{array}{c|c}
\left(\partial_{z}^{2}-k^{2}\right)\left(1-\frac{h}{2} \operatorname{Pr} k^{2}+\frac{h}{2} \operatorname{Pr} \partial_{z}^{2}\right) & +h \operatorname{Pr} E k^{-1} \partial_{z} \\
\hline+h \operatorname{Pr} E k^{-1} \partial_{z} & 1-\frac{h}{2} \operatorname{Prk}^{2}+\frac{h}{2} \operatorname{Pr} \partial_{z}^{2}
\end{array}\right)
\end{aligned}
$$

Die Quadranten links oben und rechts unten enthalten die gleichen Teilmatrizen für das Poloidal- bzw. Toroidalfeld, die auch zur Lösung mit explizitem Coriolisterm nötig sind. Die Quadranten links unten und rechts oben stellen die Kopplung dar. Diese Matrizen werden, wie oben beschrieben, LU-zerlegt gespeichert.

Die Vektoren $\mathbf{x}^{n}$ und $\mathbf{x}^{n+1}$ enthalten für das gekopplte Gleichungssystem die ChebyshevKoeffizienten sowohl des Toroidal- als auch des Poloidalfeldes zum jetzigen bzw. gesuchten Zeitpunkt:

$$
\mathbf{x}^{n}=\left(\hat{\phi}_{0}^{n}, \ldots, \hat{\phi}_{N_{z}-1}^{n}, \hat{\psi}_{0}^{n}, \ldots, \hat{\psi}_{N_{z}-1}^{n}\right)^{T}
$$




$$
\mathbf{x}^{n+1}=\left(\hat{\phi}_{0}^{n+1}, \ldots, \hat{\phi}_{N_{z}-1}^{n+1}, \hat{\psi}_{0}^{n+1}, \ldots, \hat{\psi}_{N_{z}-1}^{n+1}\right)^{T}
$$

Gleiches gilt für die expliziten Terme der rechten Seite

$$
\mathbf{b}^{n}=\left(b_{\hat{\phi}, 0}^{n}, \ldots, b_{\hat{\phi}, N_{z}-1}^{n}, b_{\hat{\psi}, 0}^{n}, \ldots, b_{\hat{\psi}, N_{z}-1}^{n}\right)^{T}
$$

mit $b_{\hat{\phi}, i}^{n}=3 b_{\hat{\phi}, i}^{n}+b_{\hat{\phi}, i}^{n-1}$ und analog für das Toroidalfeld.

Durch die implizite Behandlung des Coriolisterms entsteht eine Matrix mit Rang $2 \cdot N_{z}$. In den einzelnen Quadranten müssen die Randbedingungen eingehalten werden. Dadurch sind für das Toroidalfeld rechts unten zwei Zeilen vorgegeben, wie in Matrix (3.25), und für das Poloidalfeld links oben sogar vier Zeilen, da es für das Poloidalfeld nach den Gleichungen (3.9a,3.9b) vier Randbedingungen gibt.

Der Nachteil des impliziten Coriolisterms ist offensichtlich: Die Matrizen haben doppelt so viele Einträge wie die einzelnen Matrizen für Toroidal- und Poloidalfeld zusammen. Dadurch erhöht sich der Speicherverbrauch und der Rechenaufwand bei der Rückersetzung mit den rechten Seiten. Es ist also entscheidend, dass man sich genau überlegt, ob der Coriolisterm die NavierStokes-Gleichung dominiert. Sollte das nicht der Fall sein, dann spart man durch eine explizite Behandlung desselben Rechenzeit. 


\section{Kapitel 4}

\section{Beschreibung des Programms}

In diesem Kapitel soll das Programm vorgestellt werden. Es basiert auf dem Programm mpplconv aus [19]. Zuerst werden die Änderungen gegenüber der Ursprungsversion beschrieben und danach der Programmaufbau/-ablauf und die Parallelisierung erklärt. Auf die Berechnung der rechten Seiten wird gesondert eingegangen.

\section{1 Änderungen am Programm}

In der Ursprungsversion [19] wurde die Strömung mit no-slip Rändern oben und unten sowie ohne Coriolisterm simuliert. Zur Implementierung der schubspannungsfreien Randbedingungen musste das Programm grundlegend überarbeitet werden. Die Matrizen wurden damals so aufgestellt, dass in den Gleichungssystemen die Randbedingung $\mathbf{u}=0$ oben und unten implizit enthalten waren. Dazu wurden Projektionsfunktionen aus [29] verwendet. Diese hatten außerdem die Eigenschaft, dass die Matrizen eine banddiagonale Struktur erhielten. Dadurch war es möglich, die LU-Zerlegung der Matrizen zu jedem Zeitschritt neu zu berechnen, da die Routinen für solche banddiagonalen Matrizen sehr schnell sind. Aus diesem Grund war es auch möglich einen dynamischen Zeitschritt zu verwenden.

Im vorliegenden Programm haben die Matrizen keine so einfache Form mehr, da die Randbedingungen explizit als Einträge in den Zeilen der Matrizen stehen. Dies erkennt man gut im Beispiel in Matrix (3.25). Allerdings hat inzwischen die Größe des zur Verfügung stehenden Hauptspeichers der Computer so zugenommen, dass es keine Probleme mehr macht, die Matrizen zu Beginn der Simulation zu zerlegen und zu speichern. Zur Zerlegung und Rückersetzung wurden die Routinen ludcmp und lubksb aus [32] verwendet. Die Rückersetzung 
einer vollbesetzten Matrix zu jedem Zeitschritt ist nur wenig langsamer als die Zerlegung und Rückersetzung einer banddiagonalen Matrix zusammen.

Eine Einschränkung ist mit diesem Verfahren aber verbunden: Eine dynamische Anpassung des Zeitschrittes ist aus Zeitgründen unvorteilhaft, weil jedes mal sämtliche Zerlegungen neu berechnet werden müssten. Deshalb wird auf einen dynamischen Zeitschritt verzichtet.

Das Programm musste auch noch um den Coriolisterm erweitert werden, was keine großen Änderungen am Code erforderte. Es wird lediglich bei der Berechnung der rechten Seiten der zusätzliche Term $2 \operatorname{PrE} k^{-1}$ addiert, wenn der Coriolisterm explizit behandelt werden soll. Soll er stattdessen implizit behandelt werden, so gibt es zwei Beiträge, wie in Abschnitt 3.3.2 gezeigt. Der eine steht mit den Werten des alten Zeitschritts auf der rechten Seite in der Matrix $\mathcal{M}^{n}$. Der andere Beitrag steht in der Matrix $\mathcal{M}^{n+1}$.

\subsection{Aufbau des Programms}

Der Aufbau des Programms soll hier einmal kurz vorgestellt werden. Er ist schematisch in Abbildung 4.1 dargestellt. Die einzelnen Blöcke werden im Folgenden erläutert:

- Initialisierung

Wird das Programm parallel ausgeführt, so wird zuerst die Parallelisierung initialisiert.

- Einlesen der Paramter

Hier werden die Simulationsparamter, zum Beispiel die Rayleigh-Zahl, Prandtl-Zahl und weitere Größen, eingelesen und an alle Prozesse verteilt. Dann werden die Variablen initialisiert, wozu z.B. auch die Komponenten $k_{x}$ und $k_{y}$ des Wellenvektors k zählen. Sind diese bekannt, dann werden die Matrizen für den Zeitschritt aufgestellt. Dies geschieht je nachdem, ob ein impliziter oder expliziter Coriolisterm gewählt wurde. Die entsprechenden Matrizen werden LU-zerlegt gespeichert.

- Einlesen der Startfelder bzw. Erzeugen zufälliger Felder

Die Startfelder, also Poloidal-, Toroidal- und Temperaturfeld, werden eingelesen, wenn eine vorherige Rechnung fortgesetzt werden soll. Ist das nicht der Fall, so werden im Temperaturfeld Zufallswerte eingesetzt, die natürlich trotzdem die Randbedingungen erfüllen müssen! Das Poloidal- bzw. Toroidalfeld werden auf Null gesetzt.

- Berechnung der rechten Seiten

In diesem Schritt werden die expliziten Anteile der rechten Seiten berechnet. Dazu ist 


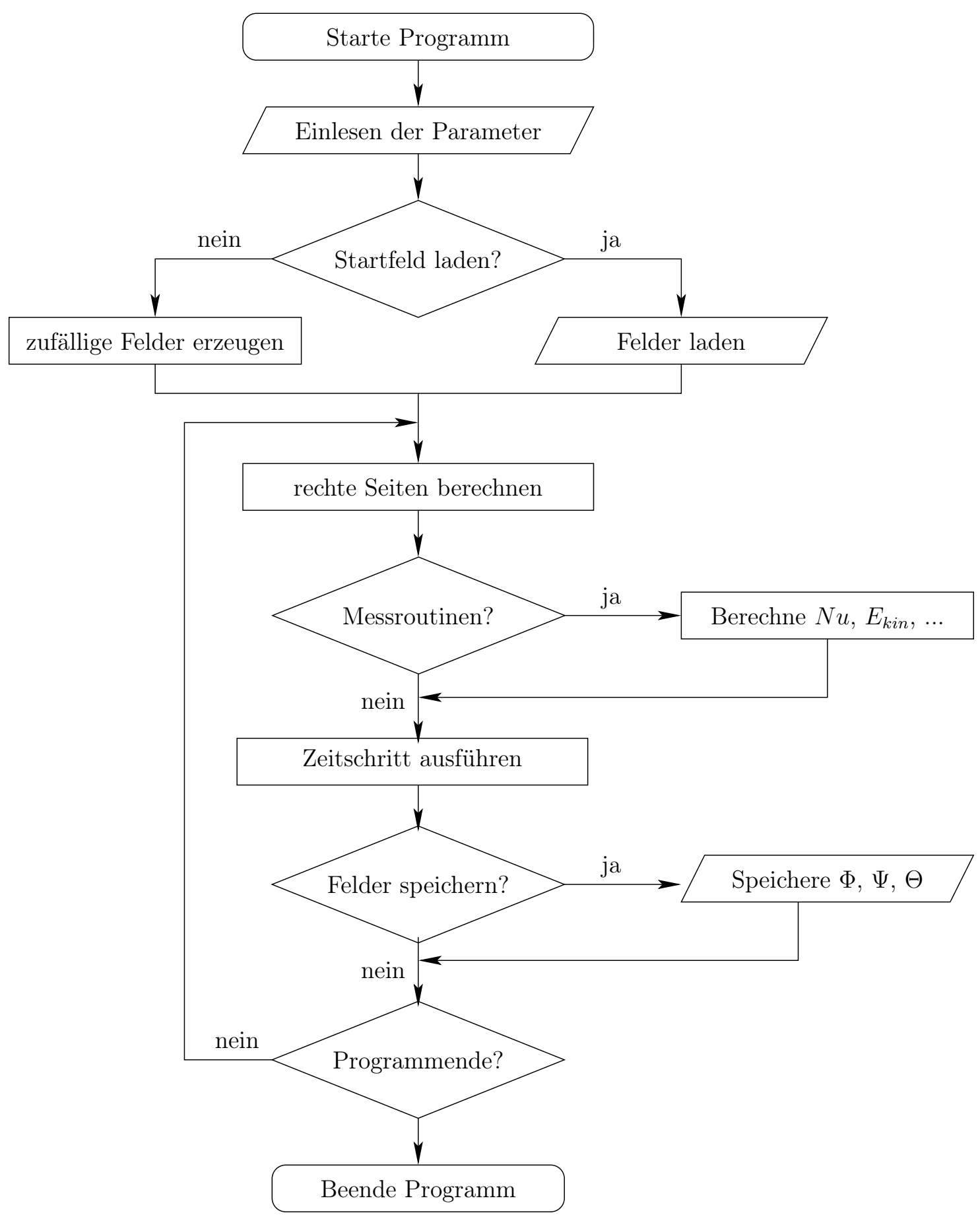

Abb. 4.1: Aufbau des Programms: Flussdiagramm mit den wichtigsten Programmschritten. 
es nötig, die Felder vom Fourierraum in den Ortsraum und zurück zu transformieren. Vorher werden im Spektralraum allerdings noch die Ableitungen der Felder berechnet. Das genaue Vorgehen ist in Abschnitt 4.4 und Abbildung 4.3 gezeigt.

Ebenfalls in diesem Schritt werden in regelmäßigen Abständen die Messroutinen aufgerufen. Dazu gehört z.B. die Berechnung der Nusselt-Zahl und der kinetischen Energie. Diverse Stastik-Routinen schreiben z.B. (laufende) Mittelwerte der Geschwindigkeitskomponenten in Dateien. Auch einzelne, vorher festgelegte Schnitte durch das Simulationsgebiet zur späteren Erstellung von Filmsequenzen können jetzt gespeichert werden.

- Zeitschritt ausführen

Jetzt wird der eigentliche Zeitschritt ausgeführt. Für alle Felder werden zu jedem k die kompletten rechten Seiten aufgestellt, bestehend aus den beiden expliziten Anteilen des jetzigen und des vorherigen Zeitschrittes und dem impliziten Anteil. Danach wird die entsprechende LU-zerlegte Matrix $\mathcal{M}^{n+1}$ aus dem Speicher geholt und mit der rechten Seite der Wert zum neuen Zeitpunkt ermittelt. Zum Schluss werden noch die aktuellen expliziten rechten Seiten für den nächsten Zeitschritt gespeichert.

- Felder speichern

Wenn es gewünscht wird, dann werden die kompletten (spektralen) Felder gespeichert, um z.B. die Rechnung an dieser Stelle zu einem späteren Zeitpunkt fortsetzen zu können.

- Programm beenden

Das Programm wird beendet, wenn die festgesetzte Anzahl an Zeitschritten ausgeführt wurde. Sollte das Programm parallel ausgeführt worden sein, so wird die MPIKommunikation vorher ebenfalls beendet.

Das Programm wird ebenfalls beendet, wenn die Simulation „explodiert“ ist. Das tritt z.B. dann auf, wenn das CFL-Kriterium in Gleichung (3.21) verletzt wird, also die maximale Geschwindigkeit für das gewählte Gitter bzw. den Zeitschritt zu groß wird. Dies ist sinnvoll, um nicht unnötig Rechenzeit für unsinnige Ergebnisse zu verschwenden.

\subsection{Parallelisierung und Speicheraufteilung}

Der Rechenaufwand einer Simulation erhöht sich, wenn das Gebiet immer feiner aufgelöst werden soll oder muss. Um die Rechenzeit in akzeptablen Grenzen zu halten, werden Teile des Programms parallel auf mehreren Rechnern (CPU-Kernen) ausgeführt. Dazu kommt das Mes- 
sage Passing Interface (kurz MPI, siehe z.B. [17]) zum Einsatz. Damit können gezielt Daten zwischen einzelnen Prozessoren ausgetauscht werden.

In Kapitel 3.2.1 wurde gesagt, dass wegen der FFT die Auflösungen $N_{x}$ bzw. $N_{y}$ immer eine Potenz von 2 sein müssen. Um nun eine möglichst gleichmäßige Auslastung der Prozessoren zu gewährleisten, wird die Anzahl der Prozessoren $N_{\text {Procs }}$ auch als eine Potenz von 2 gewählt.

Am Beispiel der Berechnung der rechten Seiten in Abschnitt 4.4 wird man sehen, dass diese sowohl im Orts- als auch im Spektralraum erfolgen müssen. Zur Berechnung im Spektralraum müssen die Daten so verteilt sein, dass ein Prozessor immer einen vertikalen Block mit allen Chebychev-Indizes kennt, siehe Abbildung 4.2. Dies ist z.B. nötig für die Auswertung der $z$-Ableitungen mit Hilfe einer Rekursionsformel.
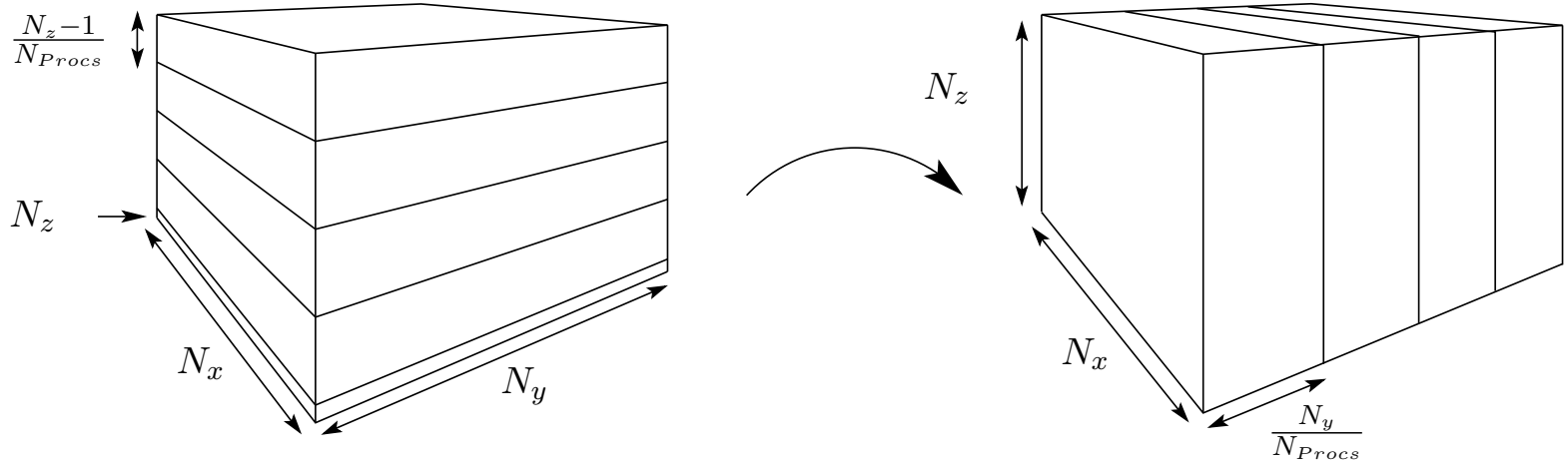

Abb. 4.2: Speicheraufteilung: Links das horizontale Speicherschema, bei der jeder Prozessor eine oder mehrere horizontale Ebenen mit allen Wellenzahlen k kennt. Die Daten liegen im Ortsraum vor, die ,vergessene“ Ebene bei $N_{z}$ ist mit eingezeichnet. Rechts das vertikale Speicherschema mit den Daten im Spektralraum.

Für den Übergang vom Spektral- zum Ortsraum müssen alle drei Raumrichtungen transformiert werden. Dies erfolgt in zwei Teilschritten: Zuerst wird mit einer Cosinus-Transformation die $z$-Richtung transformiert. Dies muss selbstverständlich in der vertikalen Aufteilung der Daten geschehen. Die beiden Fouriertransformationen in $x$ - und $y$-Richtung können so jedoch nicht durchgeführt werden, da ein Prozessor immer nur einen Ausschnitt der Wellenzahlen $k_{x}$ und $k_{y}$ kennt. Deshalb tauschen die Prozessoren die Daten per MPI aus, wie in Abbildung $4.2 \mathrm{zu}$ sehen. Ein Prozessor kennt jetzt immer für einen horizontalen Block alle Wellenzahlen und kann somit die FFT ausführen. Daraus ergibt sich, dass alle Berechnungen im Ortsraum in diesem horizontalen Speicherschema ablaufen. Der Übergang vom Orts- zum Spektralraum erfolgt in umgekehrter Reihenfolge. 
Auf eine Besonderheit muss noch eingegangen werden: Da die Aufösung in z-Richtung aufgrund der Chebychev-Polynome immer $N_{z}=2^{n}+1$ ist, müsste einer der Prozessoren $P_{i}\left(i=0 \ldots N_{\text {Procs }}-1\right)$ immer zusätzlich $\mathrm{zu}$ seinen Ebenen, die sich über die Punkte $1+i \frac{N_{z}-1}{N_{\text {Procs }}} \ldots(i+1) \frac{N_{z}-1}{N_{\text {Procs }}}$ erstrecken, die zusätzliche Ebene bei $N_{z}$ mit berechnen. Während dieser Zeit wären die übrigen Prozessoren untätig. Um das zu verhindern ,vergisst" das Programm immer eine Ebene, nämlich die unterste Ebene an der Wand (dies entspricht dem letzten Eintrag). Diese muss beim Übergang in den Spektralraum natürlich geeignet rekonstruiert werden, damit keine Information verlorengeht. Im Fall der Temperatur (bzw. der Temperaturfluktuation $\theta$ ) ist das nicht schwierig, da der Wert dort einfach rekonstruiert werden kann: Er ist Null (im Ortsraum). Dasselbe gilt für die Geschwindigkeit, wenn man feste Wände mit einer no-slip-Randbedingung verwendet.

Im vorliegenden Fall der schubspannungsfreien Randbedingung ist der Wert am Rand jedoch nicht bekannt. Lediglich von der Ableitung weiß man, dass sie Null sein muss. Aber auch damit lässt sich der Wert am Rand rekonstruieren. Dazu bedient man sich der Tatsache, dass die Cosinustransformation linear ist. Das bedeutet, dass man zuerst das falsche Feld (mit dem Wert Null als fehlenden Randwert an der letzten Stelle) transformiert. Dieser Fehler wirkt sich auf alle Chebychev-Koeffizienten aus. Insbesondere ist die Randbedingung nicht erfüllt. Um sie zu erfüllen, muss nach Gleichung (B.5) gelten:

$$
\sum_{n=0}^{N_{z}-1} \partial_{z} a_{n} T_{n}(-1)=\sum_{n=0}^{N_{z}-1} a_{n} n^{2}(-1)^{n+1} \stackrel{!}{=} 0
$$

Um die Randbedingung zu korrigieren, transformiert man einen Korrekturvektor, der nur einen Eintrag an der letzten Stelle besitzt. Die so erhaltenen Korrekturkoeffizienten dieses Vektors werden zu den jeweiligen Koeffizienten $a_{n}$ so addiert, dass Gleichung (4.1) wieder gilt.

\subsection{Berechnung der expliziten rechten Seiten}

Bevor der Zeitschritt ausgeführt werden kann, müssen die rechten Seiten $(3.4,3.5,3.7,3.11)$ berechnet werden. Die Felder liegen dazu im Spektralraum vor:

$$
\begin{aligned}
& \mathcal{R}_{\Psi}=-\hat{\mathbf{z}} \cdot[\nabla \times[(\nabla \times \mathbf{u}) \times \mathbf{u}]] \\
& \mathcal{R}_{\Phi}=-\left(\partial_{x}^{2}+\partial_{y}^{2}\right) \operatorname{Ra} \operatorname{Pr} \Theta-\hat{\mathbf{z}} \cdot[\nabla \times \nabla \times[(\nabla \times \mathbf{u}) \times \mathbf{u}]] \\
& \mathcal{R}_{\mathbf{U}}=-\langle(\nabla \times \mathbf{u}) \times \mathbf{u}\rangle_{x, y} \\
& \mathcal{R}_{\Theta}=-\left(\partial_{x}^{2}+\partial_{y}^{2}\right) \Phi-(\nabla \cdot(\mathbf{u} \Theta))
\end{aligned}
$$


Das Vorgehen, dargestellt in Abbildung 4.3, ist folgendermaßen: Zuerst werden die z-Ableitungen berechnet. Dies ist möglich, da die Felder im Spektralraum immer im vertikalen Speicherschema vorliegen. Wie in Abschnitt 4.3 erwähnt, kennt so jeder Prozessor zu einem Teil der horizontalen Wellenzahlen $k_{x}$ und $k_{y}$ immer alle Chebychev-Koeffizienten. Aus den zerlegten Feldern $\Phi$ und $\Psi$ werden nun die Geschwindigkeit u nach Gleichung 3.8 und die Rotation der Geschwindigkeit $(\nabla \times \mathbf{u})$ errechnet. Die dazu nötigen Ableitungen in $x$ - und $y$-Richtung erhält man einfach durch Multiplikation mit $i k_{x}$ bzw. $i k_{y}$. Das Ergebnis und das Temperaturfeld $\Theta$ werden wie beschrieben in den Ortsraum transformiert. Dort werden die Produkte $(\nabla \times \mathbf{u}) \times \mathbf{u}$ und $(\mathbf{u} \Theta)$ ausgerechnet und anschließend in den Spektralraum zurücktransformiert (damit ist $\mathcal{R}_{\mathbf{U}}$ bekannt). Dort werden dann das einfache bzw. doppelte Kreuzprodukt (für $\mathcal{R}_{\Psi}$ bzw. $\mathcal{R}_{\Phi}$ ) sowie $\nabla \cdot(\mathbf{u} \Theta)$ (für $\left.\mathcal{R}_{\Theta}\right)$ berechnet.

Spektralraum

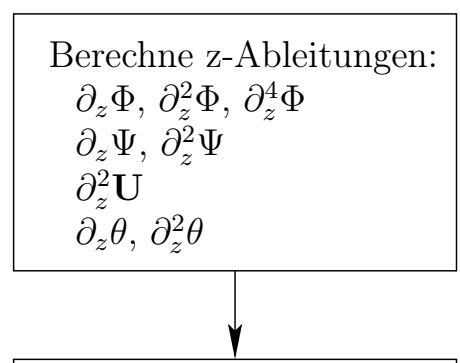

Berechne:
$\mathbf{u},(\nabla \times \mathbf{u})$

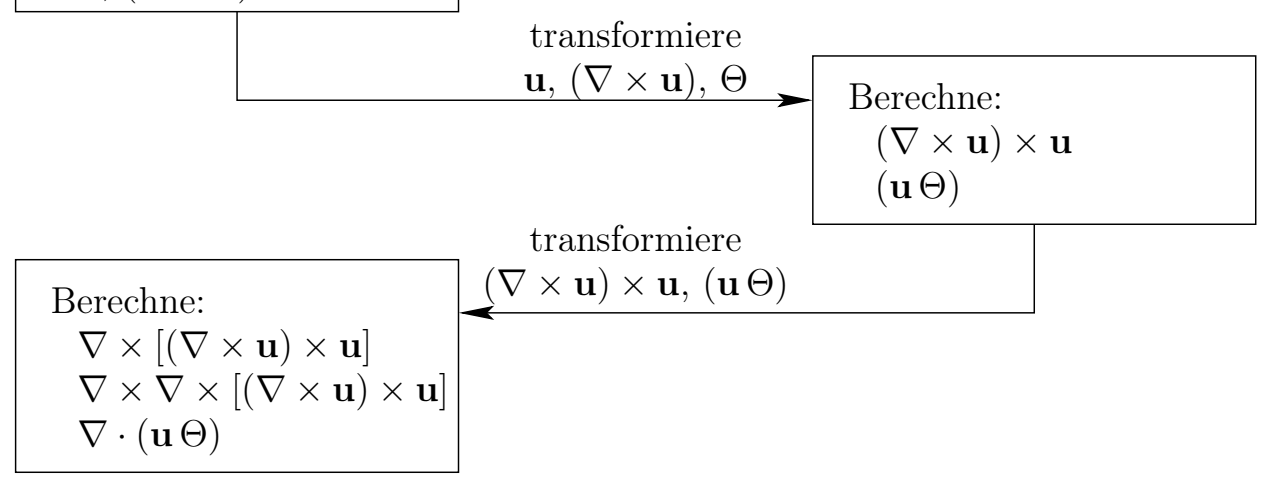

Abb. 4.3: Dargestellt ist die Aufteilung der Berechnung der expliziten rechten Seiten, die teilweise im Spektral- und teilweise im Ortsraum durchgeführt wird.

Damit sind die rechten Seiten bekannt: sie setzen sich aus dem, eben beschriebenen, expliziten Anteil, dem expliziten Anteil des vorherigen Zeitschritts und dem impliziten Anteil zusammen. Somit ist es möglich den Zeitschritt auszuführen und durch LU-Rückersetzung alle Felder zum gesuchten neuen Zeitschritt auszurechnen. 



\section{Kapitel 5}

\section{Ergebnisse}

In diesem Kapitel werden die Ergebnisse der Arbeit präsentiert. Dabei werden hauptsächlich Ergebnisse mit schubspannungsfreien Rändern gezeigt. Zur besseren Vergleichbarkeit wurden auch Simulationen mit no-slip Rändern durchgeführt, allerdings nur für einen begrenzten EkmanZahl Bereich und für nur eine Prandtl-Zahl.

Zuerst werden die Geschwindigkeitsgrenzschichten betrachtet. Für diese werden Daten mit noslip Rändern denen schubspannungsfreier Ränder gegenübergestellt, um den Unterschied zu verdeutlichen. Es wird gezeigt, dass im schubspannungsfreien Fall keine Grenzschichten entstehen.

Danach werden die Kennzahlen besprochen. Den Anfang macht die Abhängigkeit der ReynoldsZahl von der Rayleigh-Zahl. Dann werden die Ergebnisse für die Nusselt-Zahl getrennt für Simulationen ohne und mit Rotation vorgestellt. Dabei wird die Rotations-Péclet-Zahl $P e_{\text {rot }}$ eingeführt, die eine universelle Auftragung für Simulationen mit unterschiedlichen Rotationsraten ermöglicht. Für den in einer solchen Auftragung sichtbar werdenden Transitionsbereich werden diffusionsfreie Kennzahlen definiert, für die sich wiederum ein Potenzgesetz angeben lässt. Außerdem dient die Rotations-Péclet-Zahl als Kriterium zur Entscheidung, ab wann der Wärmetransport von der Rotation unbeeinflusst ist.

Danach werden die Strukturen der Strömung untersucht. Dazu zählt besonders die normierte Helizität, die als Maß für den Einfluss der Rotation auf das Fluid interpretiert wird. Sie liefert wieder ein Kriterium für den Übergang zum rotationsfreien Fall, allerdings in Abhängigkeit von der Rotations-Reynolds-Zahl $R e_{\text {rot }}$. Zuletzt werden die Spektren für Simulationen mit und ohne Rotation gegenübergestellt und eine mittlere Wellenlänge definiert. 


\subsection{Geschwindigkeitsgrenzschichten}

Wie schon der Titel der Arbeit besagt, geht es um Simulationen rotierender Rayleigh-BénardKonvektion ohne Ekman-Schichten. Dazu wurden schubspannungsfreie Randbedingungen implementiert, wie es in Abschnitt 3.3 beispielhaft vorgeführt wurde. Es soll nun gezeigt werden, wie sich diese Randbedingungen auswirken.

Die Wahl schubspannungsfreier Ränder bedingt, dass keine Geschwindigkeitsgrenzschichten in dem Sinne mehr vorkommen, als dass die Geschwindigkeit vom Wert Null am Rand auf einen bestimmten Wert im Strömungsgebiet ansteigt, wie es mit no-slip Rändern der Fall wäre. Zur Verdeutlichung sind in Abbildung 5.1 Simulationen mit no-slip (links) und schubspannungsfreien (rechts) Rändern gegenübergestellt.
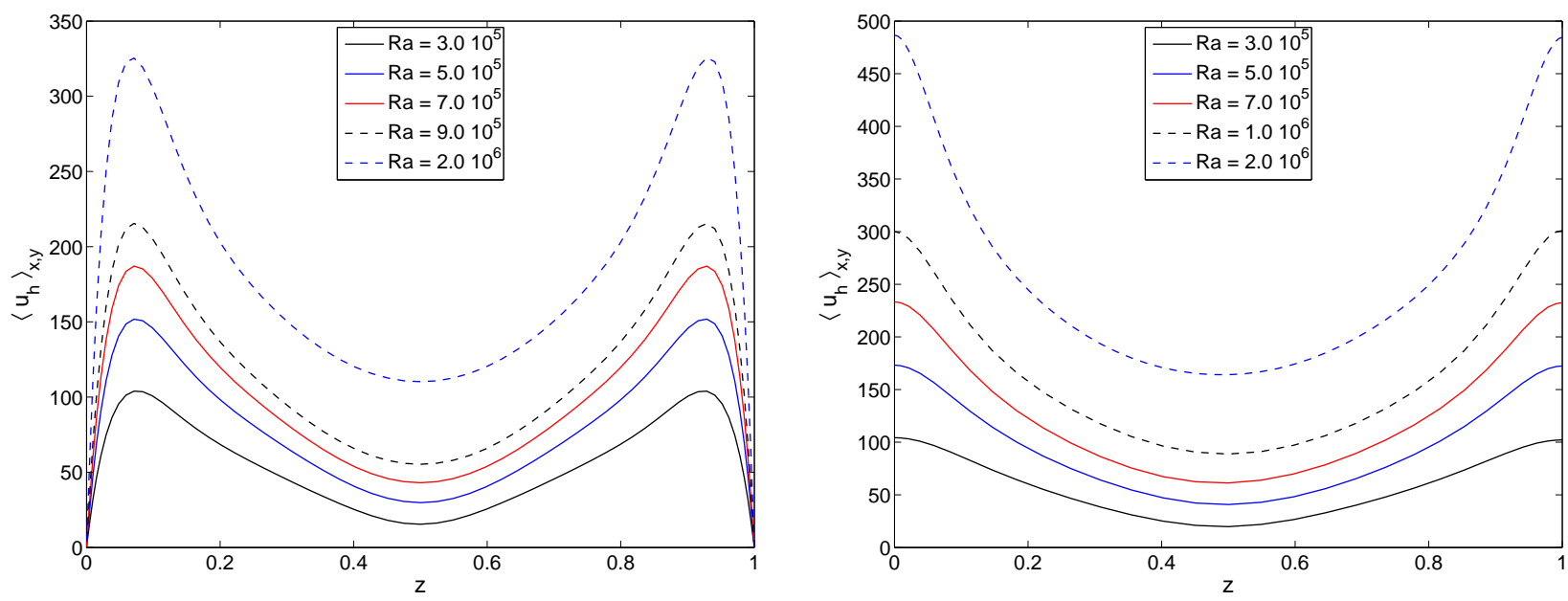

Abb. 5.1: Auftragung des Ebenenmittels der Horizontalgeschwindigkeit $\left\langle u_{h}\right\rangle_{x, y}$ über der Zellhöhe $z$ bei verschiedenen Rayleigh-Zahlen.

Links: No-slip Ränder oben und unten mit $E k=1.7 \cdot 10^{-3}$ und $\operatorname{Pr}=7.0$.

Rechts: Schubspannungsfreie Ränder oben und unten mit $E k=2.0 \cdot 10^{-3}$ und $\operatorname{Pr}=7.0$.

Mit steigender Rayleigh-Zahl steigt auch die Geschwindigkeit der Konvektionsrollen. Das ist hier durch das Ebenenmittel der Horizontalgeschwindigkeit $\left\langle u_{h}\right\rangle_{x, y}$ dargestellt. Die Horizontalgeschwindigkeit berechnet sich hierbei aus den $x$ - und $y$-Komponenten der Geschwindigkeiten:

$$
u_{h}=\sqrt{u_{x}^{2}+u_{y}^{2}}
$$

Im no-slip Fall ist die Geschwindigkeit am Rand Null und steigt bis auf einen Maximalwert an. Die z-Position dieses Maximums wird als Grenzschichtdicke $\delta_{u}$ definiert. In Abbildung 5.2 (links) ist diese Position aufgetragen. Mit steigender Geschwindigkeit wird $\delta_{u}$ kleiner. Beide Effekte (Ansteigen des Maximums von $\left\langle u_{h}\right\rangle_{x, y}$ und Verringerung von $\delta_{u}$ ) haben zur Folge, dass 
der Gradient am Rand immer größer wird, was gut in Abbildung 5.1 (links) zu sehen ist. Damit steigt auch der numerische Aufwand, da eine größere Auflösung in z-Richtung notwendig wird, um eben diesen Gradienten noch aufzulösen.

Im Fall mit no-slip Rändern hat aber auch die Rotation einen Einfluss auf die Dicke $\delta_{u}$ der Geschwindigkeitsgrenzschicht (siehe [16]), sie verhält sich wie:

$$
\delta_{u} \propto E k^{1 / 2}
$$

Auch das ist in Abbildung 5.2 (links) zu sehen. Die gleiche Abbildung zeigt rechts eine mit $E k^{0.486}$ kompensierte Auftragung. Der Exponent ist nicht $\frac{1}{2}$, wie theoretisch erwartet, aber mit 0.486 recht nahe daran. Mit kleiner werdender Ekman-Zahl wird also die Grenzschicht immer dünner. Für die kleinste Ekman-Zahl erkennt man auch, dass die Auflösung in z-Richtung nicht ausreichend war, um diese noch genügend aufzulösen.
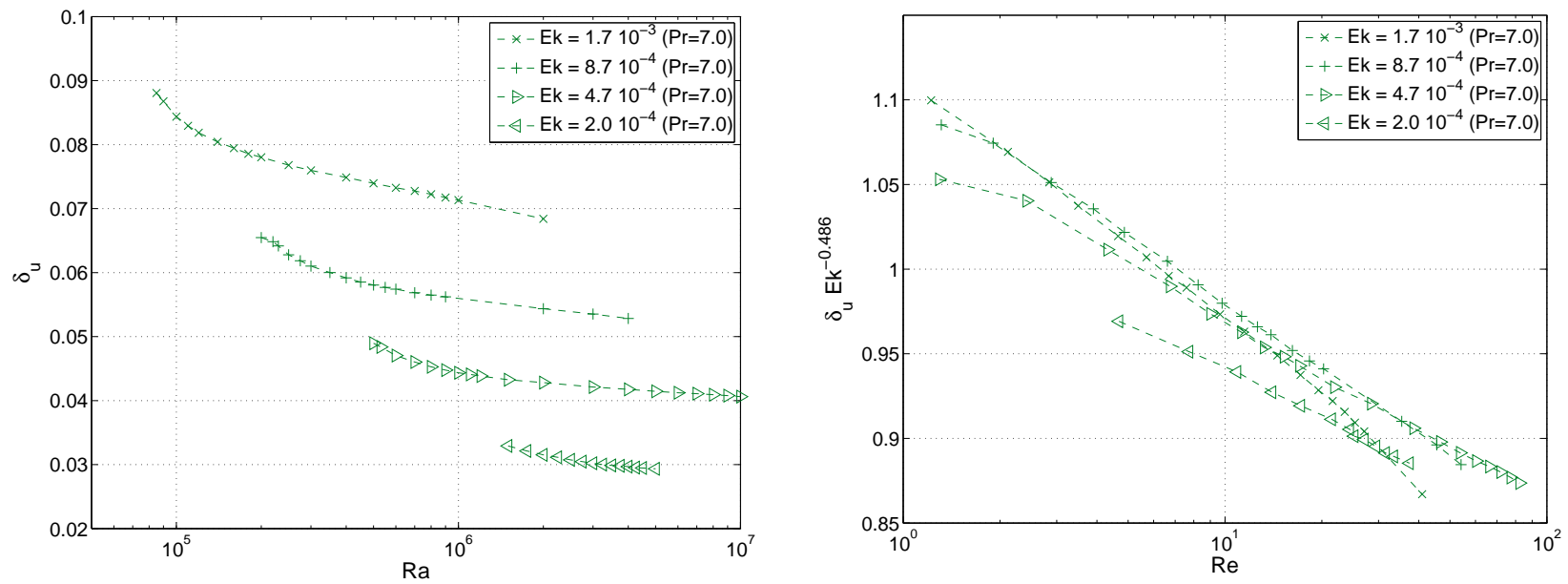

Abb. 5.2: Auftragung der Grenzschichtdicke $\delta_{u}$ über der Rayleigh-Zahl (links) und normiert mit $E k^{0.486}$ über der Reynolds-Zahl (rechts).

Für den Fall der schubspannungsfreien Ränder gibt es keine Abhängigkeit von der EkmanZahl. Nach [21] gibt es eine zu Gleichung (5.1) analoge Abhängigkeit für die Komponenten der Schubspannung, also $\varrho_{0} \nu \partial_{z} u_{x}$ und $\varrho_{0} \nu \partial_{z} u_{y}$. Allerdings gilt das nur für ein Fluid, bei dem es auf dem Rand einen Dichte- bzw. Temperaturgradienten gibt, was in dieser Arbeit nicht der Fall ist.

Um das zu verifizieren wurde das Ebenenmittel $\left\langle\left|\partial_{z} u_{h}\right|\right\rangle_{x, y}$ berechnet. Die $z$-Position, an der diese Größe das Maximum besitzt (was gleichbedeutend ist mit einem Wendepunkt im Profil der Horizontalgeschwindigkeit), wird als Definition einer Grenzschichtdicke $\delta_{u}$ für den schubspannungsfreien Fall verwendet. Abbildung 5.3 zeigt dieses Maximum, aufgetragen über der Rayleigh-Zahl. 
Wie man gut erkennt, gibt es keine Abhängikeit von der Ekman-Zahl in dieser Größe. Nahe des Onsets, wenn die Strömung noch laminar ist, erstreckt sich die Grenzschicht bis zur Zellmitte. Dort liegt der einzige Wendepunkt. Mit steigender Rayleigh-Zahl sehen die Profile dann aus wie in Abbildung 5.1 (rechts) und haben zwei Wendepunkte. Mithin sinkt die so definierte Grenzschichtdicke. Sie gleicht sich für große Rayleigh-Zahlen, wenn der Einfluss der Corioliskraft klein ist gegenüber dem Advektionsterm (die Rotation also unwichtig wird), der des nichtrotierenden Falls an.

Das rechtfertigt auch die Wahl schubspannungsfreier Ränder, wenn es um die Reduzierung des numerischen Aufwands geht. Würden die Ableitungen $\partial_{z} u_{x}, \partial_{z} u_{y}$ bzw. $\partial_{z} u_{h}$ die gleiche EkmanZahl Abhängigkeit zeigen wie die Geschwindigkeit selbst, dann hätte man nichts gewonnen: Die Ableitung der Geschwindigkeit muss natürlich ebenso durch eine genügend feine Diskretisierung aufgelöst werden wie die Geschwindigkeit selbst.

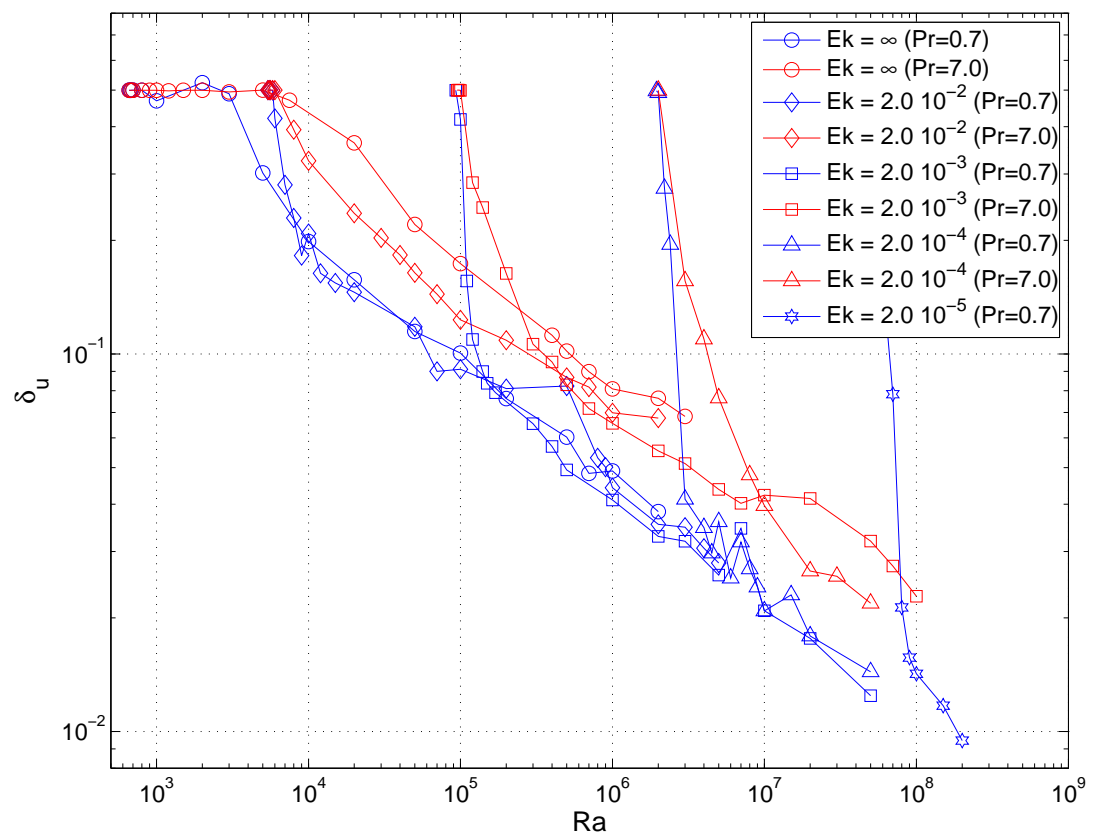

Abb. 5.3: Grenzschichtdicke $\delta_{u}$ für schubspannungsfreie Ränder, aufgetragen über der Rayleigh-Zahl.

Die schwankenden Werte dieser Auftragung rühren übrigens daher, dass die Werte aus dem Strömungsfeld zu nur einem Zeitpunkt berechnet wurden, da zu dieser Größe keine gemittelten Daten über die gesamte Simulationsdauer vorlagen. Dies wird auch in einigen anderen Auftragungen, die noch folgen werden, zu sehen sein. 


\subsection{Reynolds-Zahl}

Die Reynolds-Zahl ist ein Maß für die Geschwindigkeit der Konvektionsströmung. Die Auftragung der Reynolds-Zahl über der Rayleigh-Zahl liefert für genügend große Rayleigh-Zahlen ein Potenzgesetz $R e \propto R a^{\xi}$, wie in Abbildung $5.4 \mathrm{zu}$ sehen ist. Dies gilt für beide Prandtl-Zahlen. Die Exponenten sind $\xi=0.49$ bzw. $\xi=0.5$. Diese Exponenten wurden auch in Experimenten mit Luft [11] und Wasser [40] gefunden. Ebenso sagt die Theorie [9], [18] einen Exponenten von $\xi=0.5$ voraus. Man erkennt allerdings, dass für den Fall mit Rotation dieses Verhalten erst später erreicht wird, wenn der Einfluss der Rotation nachgelassen hat, die Rayleigh-Zahl also groß genug ist.
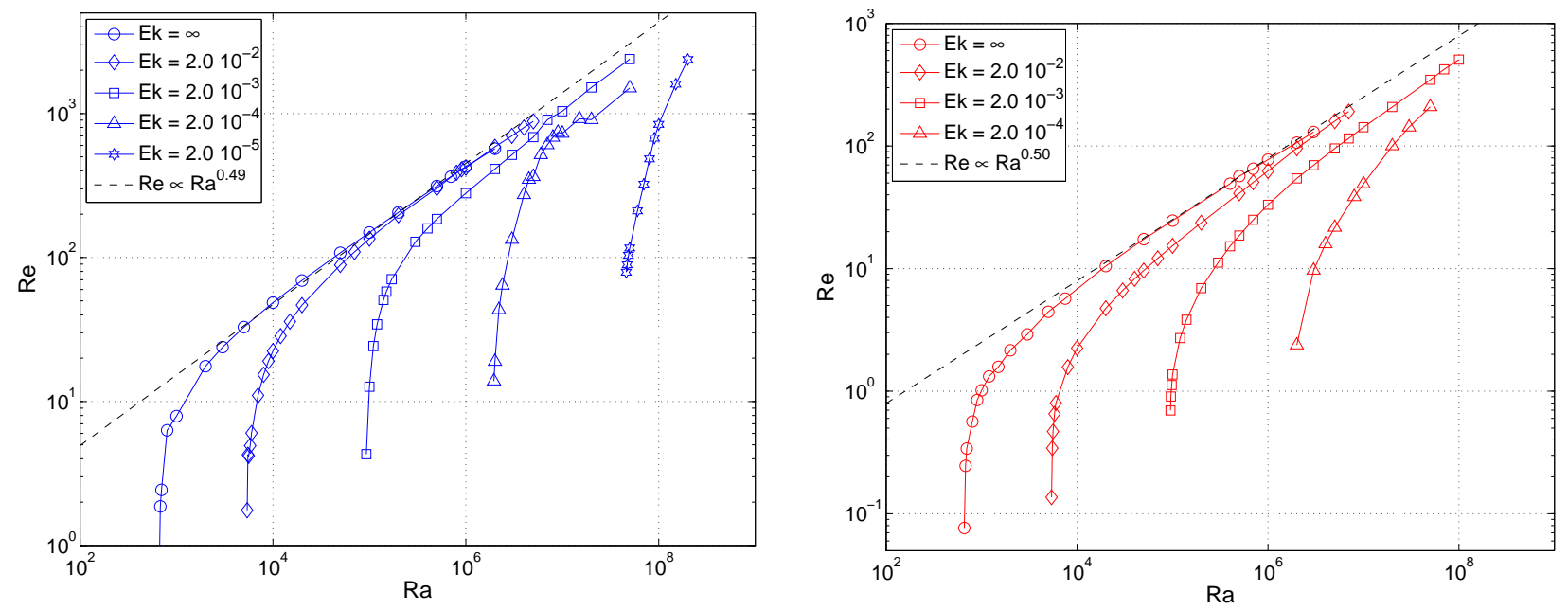

Abb. 5.4: Abhängigkeit der Reynolds-Zahl von der Rayleigh-Zahl ohne und mit Rotation (links: $\operatorname{Pr}=0.7$, rechts: $\operatorname{Pr}=7.0$ ).

In Abbildung 5.5 ist statt der Reynolds- die Péclet-Zahl $P e=\operatorname{RePr}$ über der Rayleigh-Zahl aufgetragen. Damit fallen die Kurven aus Abbildung 5.4 zusammen. Dies gilt zumindest für den Fall nahe am Onset. Für größere Rayleigh-Zahlen zeigt sich ein Offset, der auf eine (hier nicht näher untersuchte) Prandtl-Zahl Abhängigkeit hinweist. Nahe am Onset ist die Péclet-Zahl fast unabhängig von der Rayleigh-Zahl. Für genügend große Rayleigh-Zahlen zeigt die Péclet-Zahl natürlich das gleiche Verhalten wie die Reynolds-Zahl, nämlich $P e \propto R a^{1 / 2}$.

\subsection{Nusselt-Zahl}

In vielen experimentellen wie numerischen Untersuchungen wird die Nusselt-Zahl gemessen. Sie hängt im Allgemeinen von der Rayleigh-Zahl, der Prandtl-Zahl und dem Aspektverhältnis ab. 


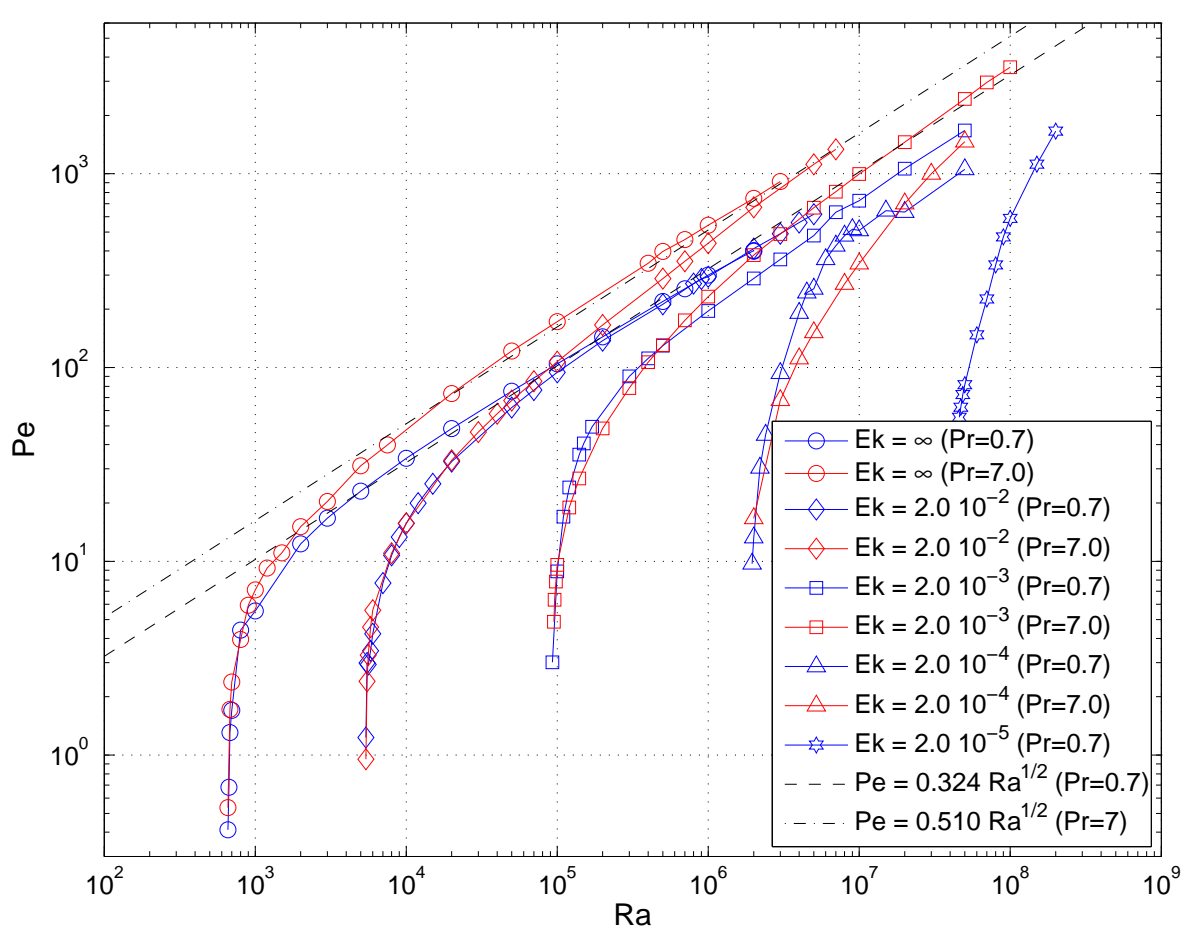

Abb. 5.5: Abhängigkeit der Péclet-Zahl von der Rayleigh-Zahl mit und ohne Rotation. Zusätzlich eingetragen sind zwei Fitgeraden mit Steigung $\frac{1}{2}$.

Im Fall mit Rotation spielt natürlich auch die Ekman-Zahl eine große Rolle (siehe Kapitel 5.3.2). Zusätzlich zu den Experimenten wird in theoretischen Arbeiten versucht, eine Abhängigkeit der Nusselt-Zahl von den genannten Größen zu finden.

In der vorliegenden Arbeit ist die Abhängigkeit vom Aspektverhältnis vernachlässigbar. Das liegt zum einen daran, dass periodische Randbedingungen in horizontaler Richtung implementiert sind. Deren Einfluss auf die Strömung ist klein im Vergleich zu festen Wänden, wie man sie z.B. in Laborexperimenten findet. Zum anderen wurde das Aspektverhältnis so gewählt, dass immer mindestens 4 Rollenpaare in der Zelle Platz fanden. Zusammen entspricht das annähernd einer unendlich ausgedehnten Zelle.

Eine Abhängigkeit von der Prandtl-Zahl wird nicht untersucht, da nur Simulationen zu zwei verschiedenen Prandtl-Zahlen $(P r=0.7$ und 7.0) durchgeführt wurden. Es bleibt also die Abhängigkeit von der Rayleigh-Zahl. Nach experimentellen und theoretischen Beobachtungen zeigt sich, dass man diese durch ein Potenzgesetz beschreiben kann:

$$
N u=N u(R a, P r, \Gamma) \propto R a^{\gamma}
$$

Der Exponent sollte (siehe z.B. [9]) ungefähr $\gamma \approx \frac{2}{7}$ sein. Allerdings ist er nicht fest, sondern 
abhängig von der Prandtl-Zahl (siehe [13] für kleine bzw. [37] für große Prandtl-Zahlen) und der Rayleigh-Zahl. Bei letzterer kommt es besonders auf den untersuchten Bereich an. Es werden Exponenten im Bereich von $\frac{1}{4}$ [22] bis $\frac{1}{3}$ [15] gefunden. Eine neuere Theorie [18] errechnet aus der Überlagerung zweier Regimes mit den Exponenten $\frac{1}{4}$ und $\frac{1}{3}$ ebenfalls eine Skalierung mit $\gamma \approx \frac{2}{7}$.

\subsubsection{Nusselt-Zahl ohne Rotation}

Die Simulationen dieser Arbeit führen zum gleichen Ergebnis, wie Abbildung 5.6 zeigt. Es ist zu erkennen, dass der Exponent $\gamma \approx \frac{2}{7}$ für die große Prandtl-Zahl $(\operatorname{Pr}=7.0)$ erst für größere Rayleigh-Zahlen erreicht wird.

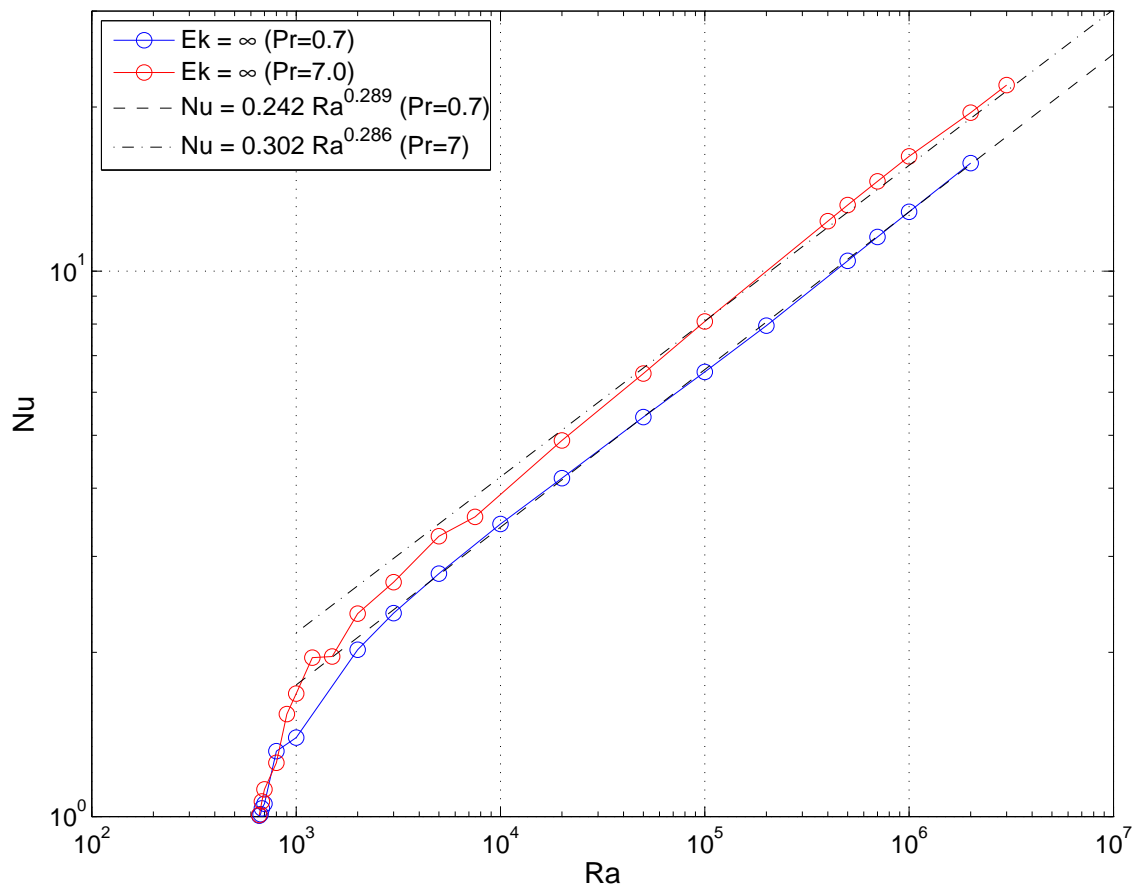

Abb. 5.6: Wärmetransport in Abhängigkeit der Rayleigh-Zahl für den Fall ohne Rotation.

Interessant ist auch die Untersuchung der rein konvektiv transportierten Wärme, also der Größe $N u-1$. Diese wird in Abbildung 5.7 ebenfalls über der Rayleigh-Zahl aufgetragen. Man erkennt sehr gut, dass sich das Potenzgesetz mit einem Exponenten von $\frac{1}{3}$ angeben lässt:

$$
N u-1 \propto R a^{1 / 3}
$$

Dasselbe Ergebnis finden auch Experimente in [20] für einen Bereich, der dort „soft turbulence“ genannt wird. Die Rayleigh-Zahlen dafür liegen im Bereich $5 \cdot 10^{5} \leq R a \leq 4 \cdot 10^{7}$. Die hier 
gezeigten Ergebnisse liegen, auch noch für kleinere Rayleigh-Zahlen, gut auf dem Fit. Die Prandtl-Zahl-Abhängigkeit wie in Abbildung 5.6 ist wieder erkennbar.

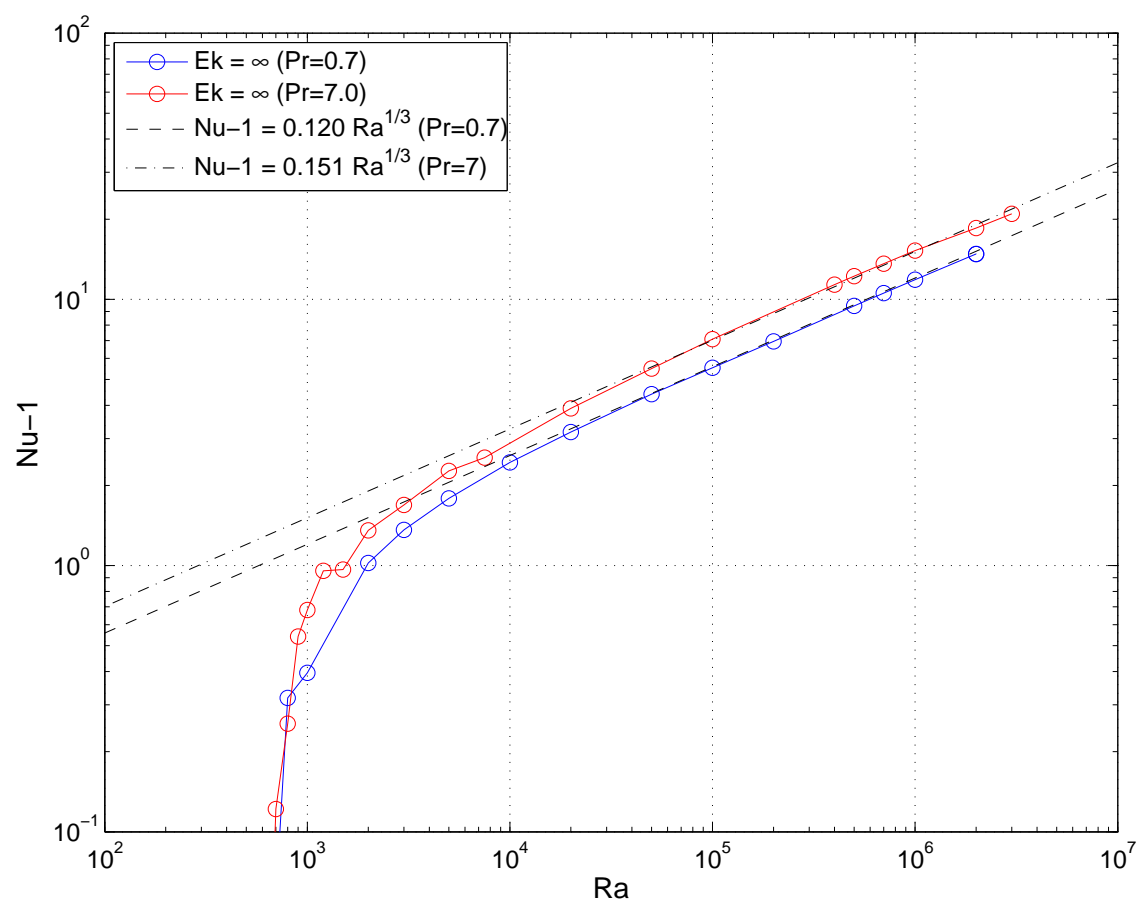

Abb. 5.7: Konvektiv transportierte Wärme $N u-1$ in Abhängigkeit der Rayleigh-Zahl für den Fall ohne Rotation.

Für noch größere Rayleigh-Zahlen erwartet man nach [20] ein Potenzgesetz

$$
N u-1 \propto R a^{2 / 7}
$$

Bei diesen Rayleigh-Zahlen ist allerdings die Nusselt-Zahl schon so groß, dass man die Näherung $N u-1 \approx N u$ annehmen kann. Damit erhält man wieder das oben beschriebene Potenzgesetz mit dem bekannten Exponenten $\frac{2}{7}$.

Die rein konvektiv transportierte Wärme wird nun über der Péclet-Zahl aufgetragen. Dies ist in Abbildung $5.8 \mathrm{zu}$ sehen. Diese Auftragung zeigt für den rotationsfreien Fall eine sehr einfache Abhängigkeit, der Graph ist zweigeteilt. Fittet man an beide Abschnitte jeweils Geraden, so ergibt sich für kleine Péclet-Zahlen gerade eine Steigung von 2, für große Péclet-Zahlen ist sie $\frac{2}{3}$. Als Übergang kann man den Schnittpunkt der beiden Fitgeraden definieren, er liegt damit bei $P e \approx 6$.

Der Fall der kleinen Péclet-Zahlen lässt sich mithilfe der kinetischen Energiedissipation erklären 
(siehe Anhang C für eine ausführliche Herleitung):

$$
R a(N u-1)=\epsilon_{u}=\frac{1}{V} \int\left\langle\left(\partial_{j} u_{i}\right)\left(\partial_{j} u_{i}\right)\right\rangle_{t} d V
$$

Für die laminare Strömung nahe am Onset identifiziert man die Geschwindigkeit nach Gleichung (2.8) mit $R e P r=P e$. Die einzig relevante Länge ist in diesem Bereich die Zellhöhe $d$, die im dimensionslosen Fall gerade 1 ist und damit entfällt. Die Variation der Rayleigh-Zahl spielt in diesem Bereich keine Rolle, sie kann als nahezu konstant angesehen werden. Dies ist auch gut in Abbildung $5.5 \mathrm{zu}$ erkennen.

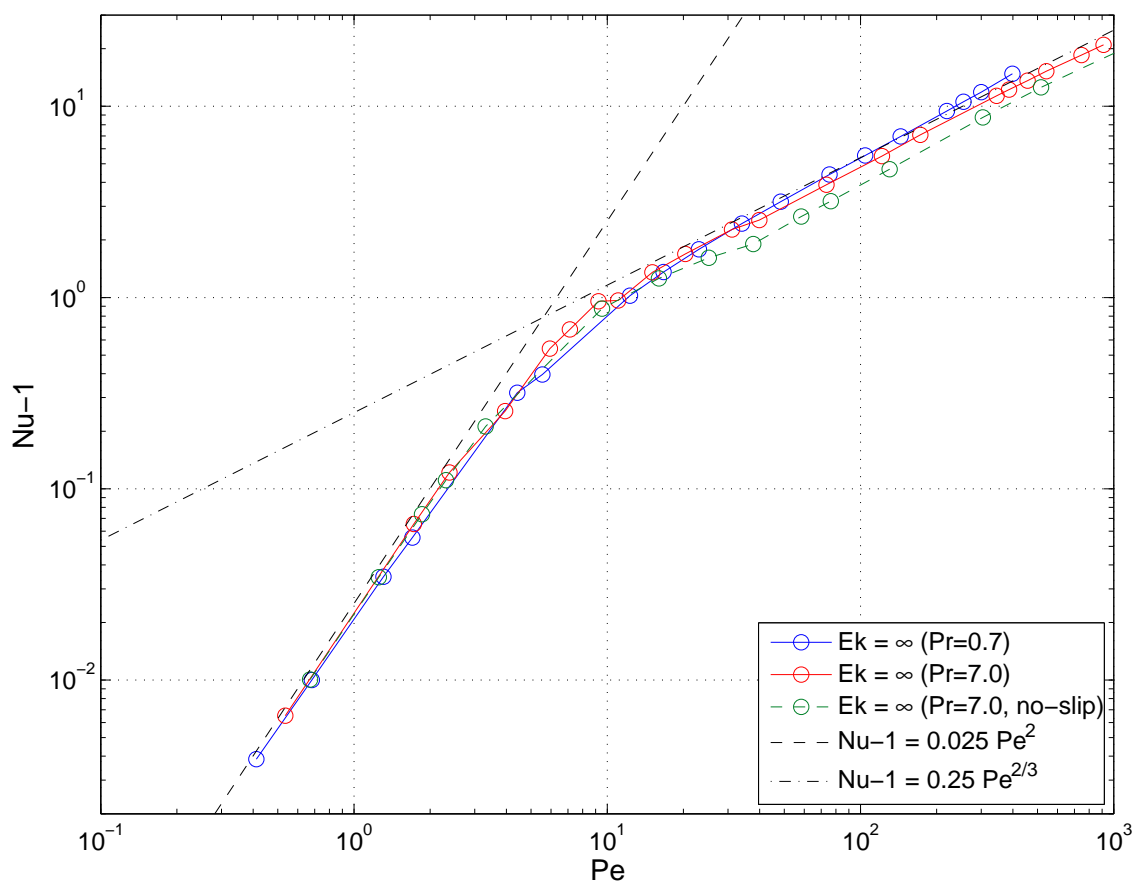

Abb. 5.8: Konvektiv transportierte Wärme in Abhängigkeit der Péclet-Zahl für den Fall ohne Rotation. Gezeigt sind zusätzlich Ergebnisse von Simulationen mit no-slip Rändern oben und unten.

Also reduziert sich Gleichung (5.4) auf:

$$
N u-1 \propto P e^{2}
$$

Für die Steigung $\frac{2}{3}$ bei großen Péclet-Zahlen lässt sich eine Erklärung herleiten aus Gleichung (5.2). Bedenkt man, dass (Reynolds- bzw.) Péclet- und Rayleigh-Zahl zusammenhängen wie $P e \propto R a^{1 / 2}$ (siehe Abschnitt 5.2), so folgt:

$$
N u-1 \propto R a^{1 / 3} \Rightarrow N u-1 \propto P e^{2 / 3}
$$


Kapitel 5. Ergebnisse

Dieses Potenzgesetz ist, für den hier betrachteten Bereich der großen Péclet-Zahlen, gut erfüllt. Es ist allerdings nicht auszuschließen, dass sich in Abbildung 5.8 für noch größere Péclet-Zahlen ein Bereich anschließt, der ein anderes Potenzgesetz aufweist, also für den Bereich, in dem statt Gleichung (5.2) nun Gleichung (5.3) gilt.

Eine Aufspaltung nach Prandtl-Zahlen, wie sie bei den Auftragungen über der Rayleigh-Zahl zu erkennen war, gibt es in der Auftragung der konvektiv transportierten Wärme über der Péclet-Zahl nicht mehr.

In Abbildung 5.8 sind zusätzlich noch Ergebnisse von Simulationen mit no-slip Rändern oben und unten eingetragen. Diese Simulationen zeigen die gleichen, eben besprochenen Potenzgesetze. Abgesehen von einem unterschiedlichen Vorfaktor ist die Auftragung $N u-1$ über $P e$ ist also universell und unabhängig von den Randbedingungen.

\subsubsection{Nusselt-Zahl mit Rotation}

Im Fall mit Rotation sieht der Velauf der Nusselt-Zahl für die verschiedenen Ekman-Zahlen aus wie in Abbildung 5.9.

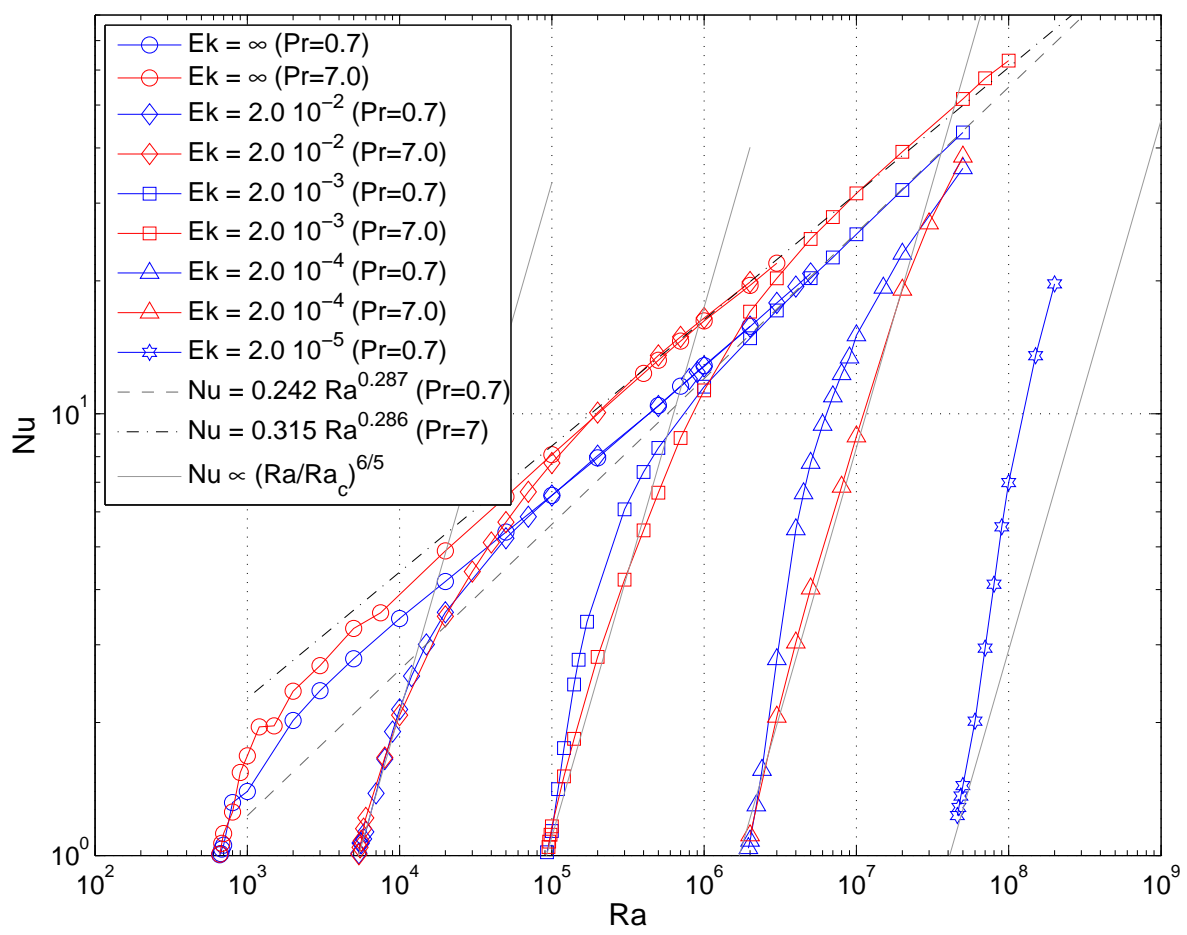

Abb. 5.9: Wärmetransport in Abhängigkeit der Rayleigh-Zahl ohne und mit Rotation. 
Man erkennt, dass mit kleiner werdender Ekman-Zahl der Onset zu größeren Rayleigh-Zahlen hin verschoben ist, wie in Abschnitt 2.3 dargelegt wurde. Die Rotation führt dazu, dass sich das Verhalten des Wärmetransports offensichtlich in zwei Bereiche unterteilt. Zum einen ist es das rotationsdominierte Regime nahe des Onsets. Wenn die Rayleigh-Zahl noch nicht viel größer als die kritische Rayleigh-Zahl ist (also die Strömung nur schwach überkritisch ist), dann ist in der Navier-Stokes-Gleichung (2.1a) der Coriolisterm größer als der advektive Term. In diesem Bereich steigt die Nusselt-Zahl steil an (siehe [24]), es gilt:

$$
N u=\left(\frac{R a}{R a_{c}}\right)^{6 / 5}
$$

Dieses Potenzgesetz ist in Abbildung 5.9 mit eingetragen. Man sieht, dass es für die große Prandtl-Zahl $\operatorname{Pr}=7.0$ gut erfüllt ist. Für die kleine Prandtl-Zahl $\operatorname{Pr}=0.7$ müsste der Exponent etwas größer sein. Es gibt also auch hier, wie schon bei der Reynolds-Zahl, eine Abhängigkeit von der Prandtl-Zahl. Vergleichbare Simulationen aus [25] zeigen auch dieses Verhalten.

Zum anderen gibt es das nicht-rotationsdominierte Regime. Wenn der advektive Term der Navier-Stokes-Gleichung den Coriolisterm überwiegt, dann wird die Rotation unwichtig. Die Strömung sollte sich dann so verhalten, als wäre keine Rotation vorhanden. Das ist in der Tat so, wie man gut erkennt: Das Verhalten der Nusselt-Zahl zeigt, wie für den nicht-rotierenden Fall, einen Exponenten von $\gamma \approx \frac{2}{7}$. Der Wärmetransport entspricht also den Werten des nichtrotierenden Falls.

Die Kurven der Nusselt-Zahl in Abbildung 5.9 sind separiert. Zum einen entlang der Abszisse aufgrund der Ekman-Zahl-Abhängigkeit des Onsets und zum anderen entlang der Ordinate aufgrund der Prandtl-Zahl-Abhängigkeit. Dadurch fallen die Kurven weder für den rotationsdominierten noch für den rotationsfreien Bereich zusammen.

Es liegt nahe, eine Auftragung wie in Abbildung $5.8 \mathrm{zu}$ suchen, in der die Kurven für den Wärmetransport zusammenfallen. Dies erreicht man, wie in Abbildung 5.10 zu sehen, indem man auf der Ordinate wieder die konvektiv transportierte Wärme $(N u-1) E k^{1 / 3}$ (modifiziert mit einem rotationsabhängigen Faktor) und auf der Abszisse die Rotations-Péclet-Zahl $P e_{\text {rot }}$ aufträgt.

Die Rotations-Péclet-Zahl $P e_{\text {rot }}$ soll definiert sein als:

$$
P e_{r o t}=\operatorname{Re} \operatorname{Pr} E k^{1 / 2}=\frac{U}{\kappa} \sqrt{\frac{\nu}{\Omega}}
$$

Sie wird im Gegensatz zur Péclet-Zahl, die mit der Zellhöhe als Referenzlänge gebildet wird, mit einer Länge proportional zu $E k^{1 / 2}$ gebildet. Dies ist die Dicke der Ekman-Schicht, wie man sie in 
rotierenden Konvektionsströmungen mit Grenzschichten vorfindet. Dies wurde in Abschnitt 5.1 gezeigt. Es wurde aber ebenfalls gezeigt, dass im vorliegenden Fall mit schubspannungsfreien Randschichten solche Grenzschichten nicht auftreten. Trotzdem zeigt der Wärmetransport für verschiedene Ekman-Zahlen eine gute Übereinstimmung, wenn man ihn über einer mit dieser Länge gebildeten Péclet-Zahl aufträgt. Dies ist in Abbildung 5.10 zu sehen.

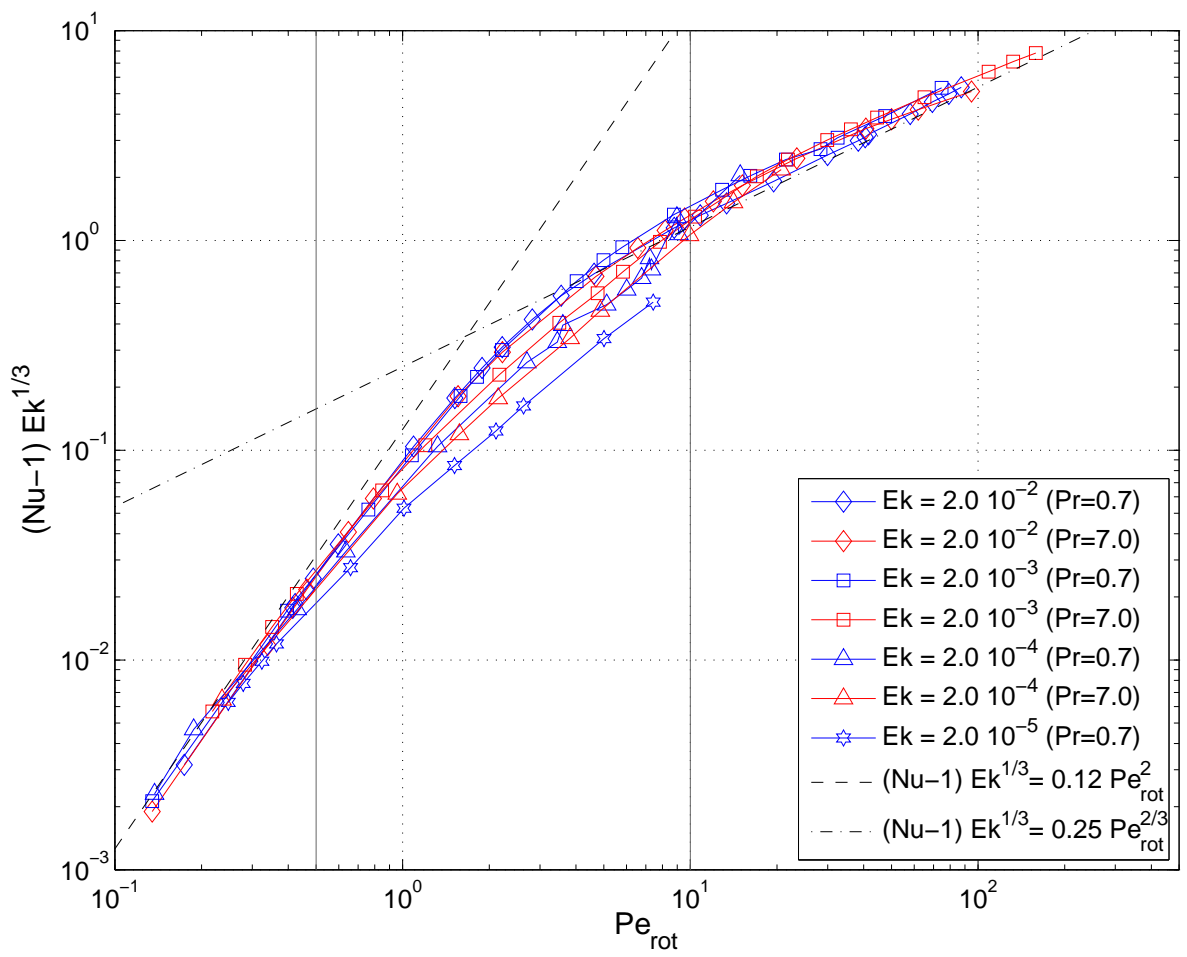

Abb. 5.10: Konvektiv transportierte Wärme in Abhängigkeit der Rotations-Péclet-Zahl für Simulationen mit schubspannungsfreien Rändern oben und unten.

Im rotationsfreien Fall in Abbildung 5.8 sah man einen zweigeteilten Graphen. In dieser Auftragung in Abbildung 5.10 erkennt man stattdessen eine Aufteilung in drei Bereiche: Erstens einen laminaren Bereich für $P e_{r o t} \leq 0.5$ nahe des Onsets, wo die Rotation dominiert. Zweitens einen rotationsfreien Bereich für $P e_{r o t} \geq 10$, wo die Rotation unwichtig wird. Und drittens einen Transitionsbereich dazwischen für $0.5 \leq P e_{r o t} \leq 10$, in dem die Kurven für verschiedene Rotationsraten nicht mehr zusammenfallen. Dessen Bedeutung wird in Abschnitt 5.3.3 ausführlich diskutiert werden.

An die ersten beiden Bereiche können wieder, wie im rotationsfreien Fall, Fitgeraden angepasst werden. Die Steigungen sind wieder die gleichen, nämlich 2 für $P e_{\text {rot }} \leq 0.5$ und $\frac{2}{3}$ für $P e_{\text {rot }} \geq 10$. Die Steigung für den rotationsdominierten Bereich nahe des Onsets ergibt sich wieder aus der 
kinetischen Energiedissipation, siehe Gleichung (5.4):

$$
R a(N u-1)=\epsilon_{u}=\frac{1}{V} \int\left\langle\left(\partial_{j} u_{i}\right)\left(\partial_{j} u_{i}\right)\right\rangle_{t} d V
$$

Die Geschwindigkeit ist nach Gleichung (2.8) wieder $\operatorname{RePr}=P e$. Die vorherrschende Länge ist in diesem Bereich nach Gleichung (2.10) allerdings die Onset-Wellenlänge $\lambda_{c} \propto E k^{1 / 3}$. Analog dazu lässt sich die Rayleigh-Zahl nach Gleichung (2.9) ausdrücken als $R a_{c} \propto E k^{-4 / 3}$. Damit ist obige Gleichung:

$$
(N u-1) E k^{1 / 3} \propto P e_{r o t}^{2} \quad \Longrightarrow \quad N u-1 \propto P e^{2} E k^{2 / 3}
$$

Die Abhängigkeit für den rotationsfreien Fall lässt sich nicht durch eine einfache Gleichung herleiten. Allerdings ergibt die Umrechnung, dass die Nusselt-Zahl unabhängig von der EkmanZahl ist, wie man es für diesen Bereich erwarten würde:

$$
(N u-1) E k^{1 / 3} \propto P e_{\text {rot }}^{2 / 3} \Longrightarrow N u-1 \propto P e^{2 / 3}
$$

Darüber hinaus wurde der Offset der Fitgeraden identisch mit dem des rotationsfreien Falls gewählt. Wie man sieht, folgen die Werte gut diesem Fit, auch wenn sie systematisch leicht darüber liegen.
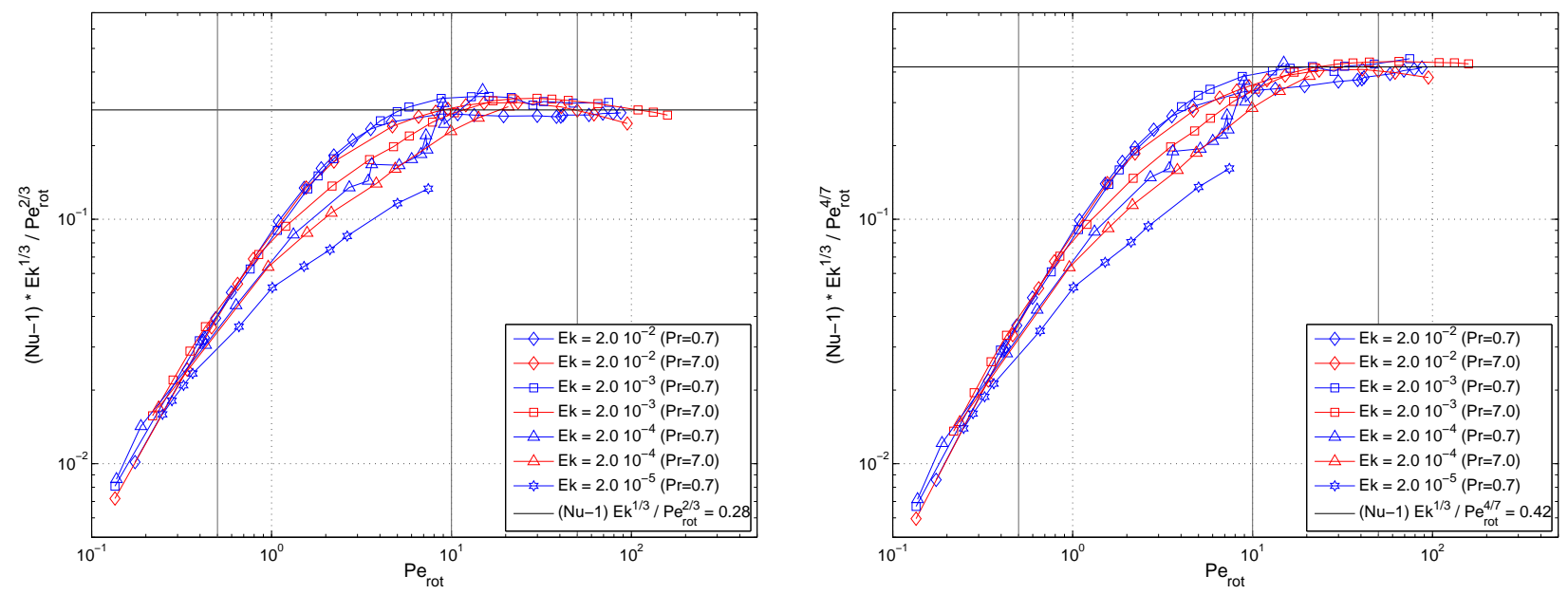

Abb. 5.11: Konvektiv transportierte Wärme (wie in Abbildung 5.10), kompensiert mit $P e_{\text {rot }}^{2 / 3}$ (links) bzw. $P e_{\text {rot }}^{4 / 7}$ (rechts), aufgetragen über $P e_{\text {rot }}$.

In Abbildung 5.10 lässt sich eventuell noch ein vierter Bereich ausmachen. Für sehr große Rotations-Péclet-Zahlen $\left(P e_{\text {rot }}>50\right)$ scheint eher ein Fit mit dem Exponenten $\frac{4}{7}$ angebracht. Dies kommt daher, dass dort (wie schon im vorigen Abschnitt für den Fall ohne Rotation angesprochen) das Potenzgesetz nicht mehr $N u-1 \propto R a^{1 / 3}$, sondern $N u-1 \approx N u \propto R a^{2 / 7}$ 
gilt. In Abbildung 5.11 ist der Graph aus Abbildung 5.10 nochmal dargestellt. Dieses mal ist er allerdings kompensiert mit $P e_{\text {rot }}^{2 / 3}$ (links) bzw. $P e_{\text {rot }}^{4 / 7}$ (rechts). Leider sind im Bereich $P e_{r o t}>50$ nur wenige Simulationsergebnisse vorhanden, so dass eine endgültige Aussage über den Exponenten in diesem Bereich schwer ist.

Abbildung 5.12 zeigt Ergebnisse für Simulationen mit no-slip Rändern oben und unten. Allerdings ist der untersuchte Bereich von Ekman- und damit Rotations-Péclet-Zahlen beschränkt.

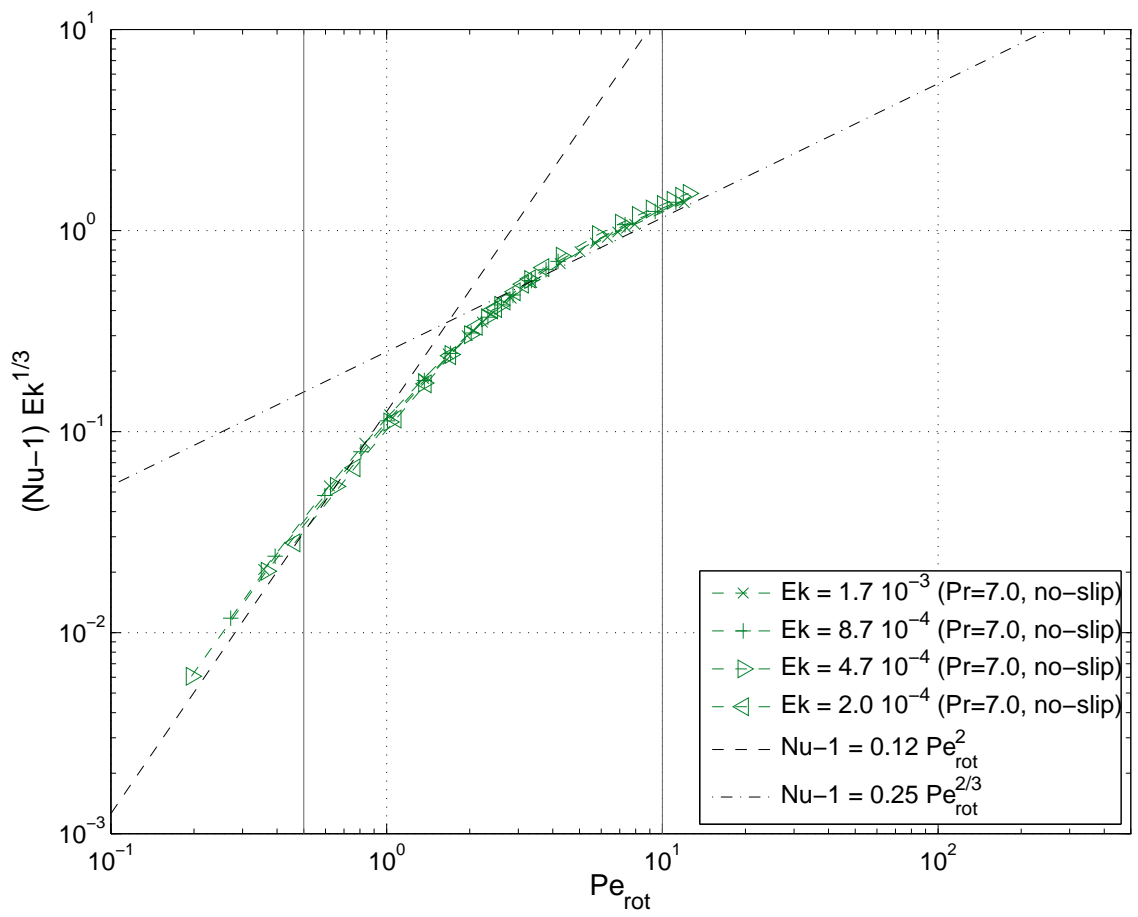

Abb. 5.12: Konvektiv transportierte Wärme in Abhängigkeit der Rotations-Péclet-Zahl für Simulationen mit no-slip Rändern oben und unten.

Trotzdem erkennt man gut, dass der konvektive Wärmetransport das gleiche Verhalten zeigt wie mit schubspannungsfreien Rändern in Abbildung 5.10. Auch die eingezeichneten Fitgeraden haben die gleichen Parameter. Die Aufspaltung nach Ekman-Zahlen im Übergangsbereich $0.5 \leq$ $P e_{\text {rot }} \leq 10$ ist hingegen nicht $\mathrm{zu}$ sehen. $\mathrm{Ob}$ das an den Randbedingungen oder dem begrenzten Bereich der untersuchten Ekman-Zahlen liegt, lässt sich nicht eindeutig beantworten. Dessen ungeachtet ist die Auftragung, wie schon Abbildung 5.8, universell für das untersuchte System. An dieser Stelle sollen kurz die Temperaturgrenzschichten gezeigt werden. Man erwartet, dass die Grenzschichtdicke sich im Vergleich zum rotationsfreien Fall nicht ändert, wenn Rotation ins Spiel kommt. Die Corioliskraft wirkt sich in der Navier-Stokes-Gleichung nur auf die 
Geschwindigkeit nach Gleichung (2.1a), aber nicht auf die Temperatur nach Gleichung (2.1b) aus.

Zur Bestimmung der Temperaturgrenzschicht kann man unter anderem das Ebenenmmittel der Temperatur verwenden. Man geht davon aus, dass der Abfall der Temperatur vom Wert an der oberen bzw. unteren Platte zur mittleren Temperatur in der Zellmitte vollständig innerhalb der thermischen Grenzschicht $\delta_{T}$ erfolgt (siehe z.B. [19]). Damit ergibt sich für die Ableitung der gemittelten Temperatur an der Wand (bzw. die Steigung des Temperaturprofils dort) gerade:

$$
\partial_{z}\langle T\rangle_{x, y}=-\frac{1}{2 \delta_{T}} \Rightarrow \delta_{T}=\frac{1}{2 N u}
$$

Die Folgerung ergibt sich aus Gleichung (2.5), wenn man bedenkt, dass die $z$-Geschwindigkeit an der Wand gerade Null ist.

In Abbildung 5.13 sind, für die gleichen Parameter wie in Abbildung 5.1, die Profile von $\langle T\rangle_{x, y}$ für no-slip und schubspannungsfreie Ränder aufgetragen.
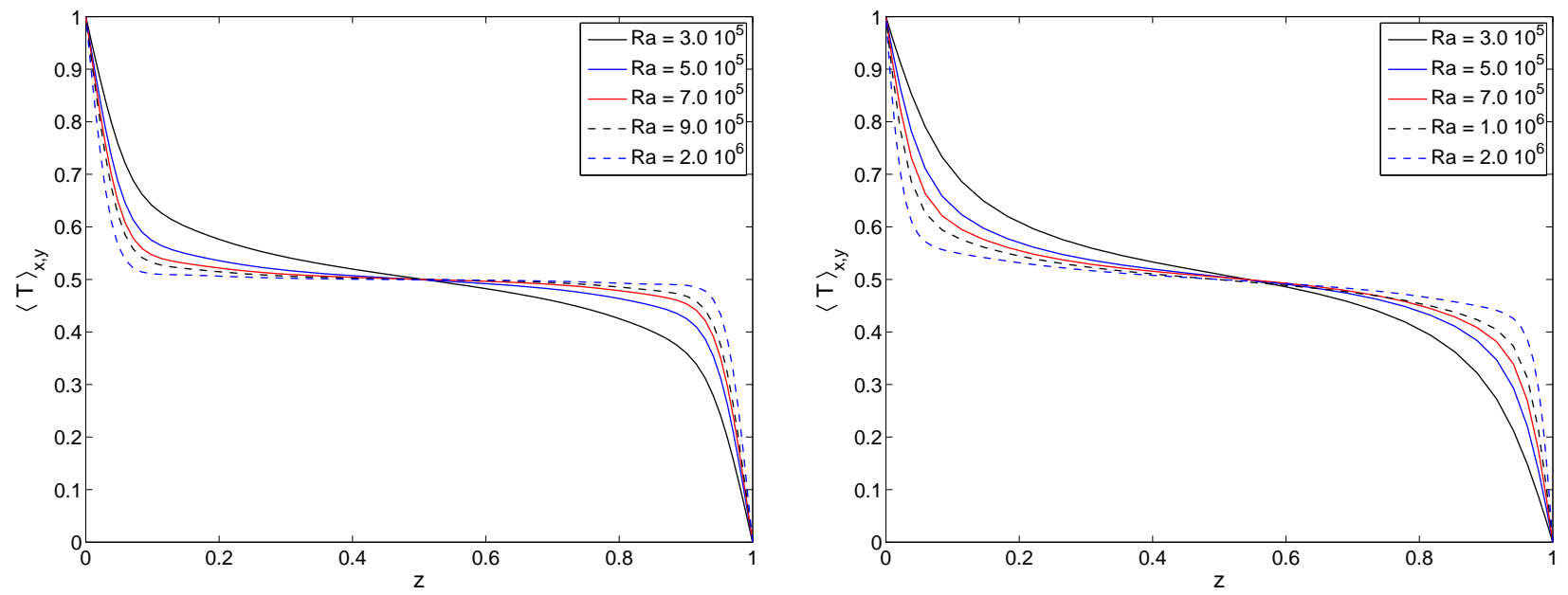

Abb. 5.13: Auftragung des Ebenenmittel der Temperatur $\langle T\rangle_{x, y}$ über der Zellhöhe $z$ bei verschiedenen Rayleigh-Zahlen.

Links: No-slip Ränder oben und unten mit $E k=1.7 \cdot 10^{-3}$ und $\operatorname{Pr}=7.0$.

Rechts: Schubspannungsfreie Ränder oben und unten mit $E k=2.0 \cdot 10^{-3}$ und $\operatorname{Pr}=7.0$.

Diese Abbildung zeigt allerdings deutlich, dass außerhalb der thermischen Grenzschicht das Temperaturprofil keineswegs konstant bei $\Delta T / 2$ liegt. Es gibt also dort noch einen Temperaturgradienten $\partial_{z}\langle T\rangle_{x, y}$. In Abbildung 5.14 ist diese Größe, gemessen in der Zellmitte bei $z=0.5$, dargestellt. Auf der Abszisse ist dieses mal $\left(R a-R a_{c}\right) / R a_{c}$ aufgetragen. Diese Größe besagt, wie überkritisch die Strömung ist.

Man erkennt mehrere Details. Die Werte für $\operatorname{Pr}=7.0$ und $E k=\infty$ liegen teilweise über der Nulllinie. Bei diesen Simulationen wurde durch die Konvektion viel Wärme von unten nach 
oben transportiert, so dass es zu einer Inversion des Temperaturprofils kommt. Aufgrund der geringen thermischen Diffusion bei der großen Prandtl-Zahl kann die Wärme nicht schnell genug verteilt bzw. an die obere Wand abgegeben werden (und analog für kaltes Fluid an der unteren Wand). Bei der Prandtl-Zahl $P r=0.7$ sieht man das nicht.

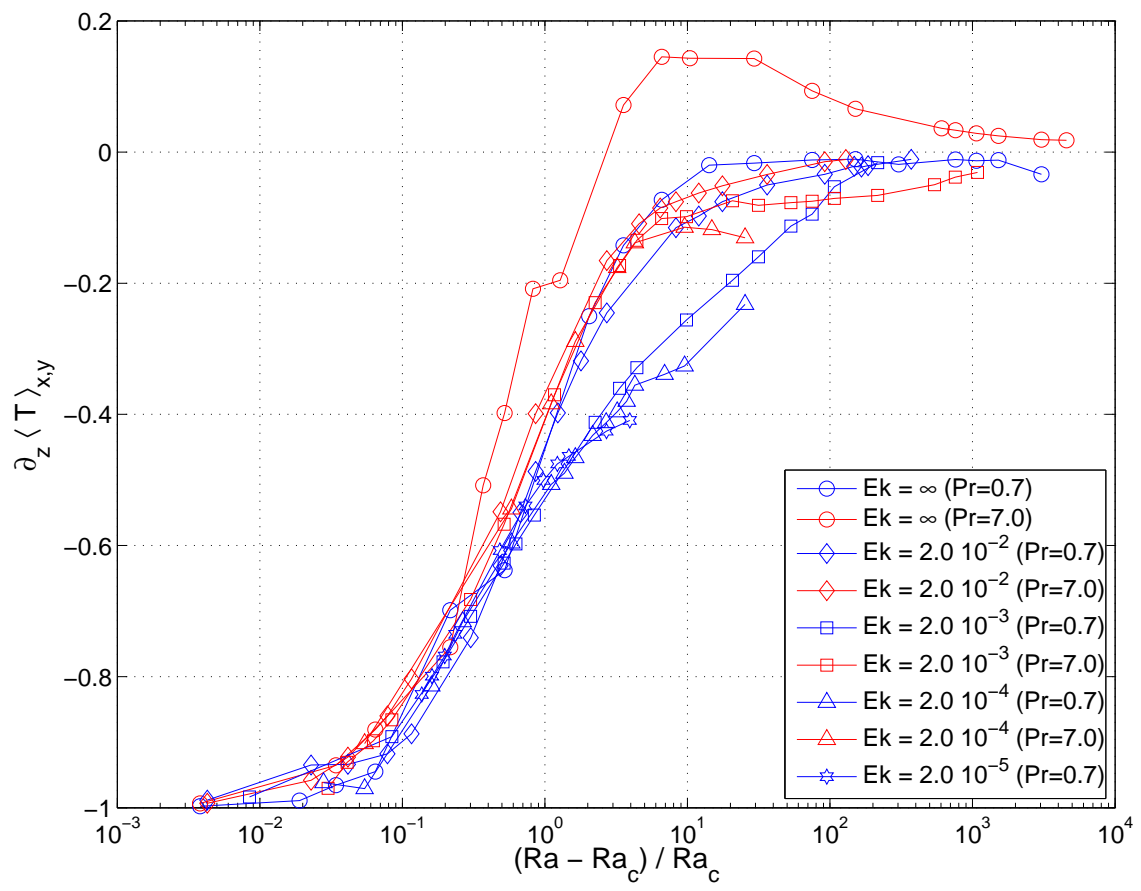

Abb. 5.14: Gradient der horizontal gemittelten Temperatur $\partial_{z}\langle T\rangle_{x, y}$ bei $z=0.5$ in der Zellmitte, aufgetragen über $\left(R a-R a_{c}\right) / R a_{c}$.

Weiter liegen die Werte für Simulationen ohne Rotation und großer Ekman-Zahl $E k=2 \cdot 10^{-2}$ gut übereinander. Die Ekman-Zahl ist so groß, dass die Rotation scheinbar kaum eine Rolle spielt. Für die übrigen Daten mit kleinen Ekman-Zahlen erkennt man eine Aufspaltung bei $\left(R a-R a_{c}\right) / R a_{c} \approx 1$. Die Kurven für $\operatorname{Pr}=0.7$ liegen deutlich unter denen für $\operatorname{Pr}=7.0$. Hier spielt wieder die thermische Diffusivität eine Rolle. Ist sie größer, so kommt es zu einem verstärkten horizontalen Wärmetransport und der Gradient wird größer. Das ist auch gut bei der Darstellung der Temperaturisoflächen in Abbildung 5.17 zu sehen, die weiter unten besprochen wird. Die Werte für beide Prandtl-Zahlen gleichen sich wieder an, wenn die Strömung etwa 100 mal überkritisch ist.

Trägt man nun die thermische Grenzschichtdicke $\delta_{T}$ über der Rayleigh-Zahl auf, ergibt sich Abbildung 5.15. Diese sieht aus wie eine gespiegelte Version von Abbildung 5.9. Nach Gleichung (5.7) ist das auch nicht anders zu erwarten gewesen. Es wurden Fits eingetragen mit den 


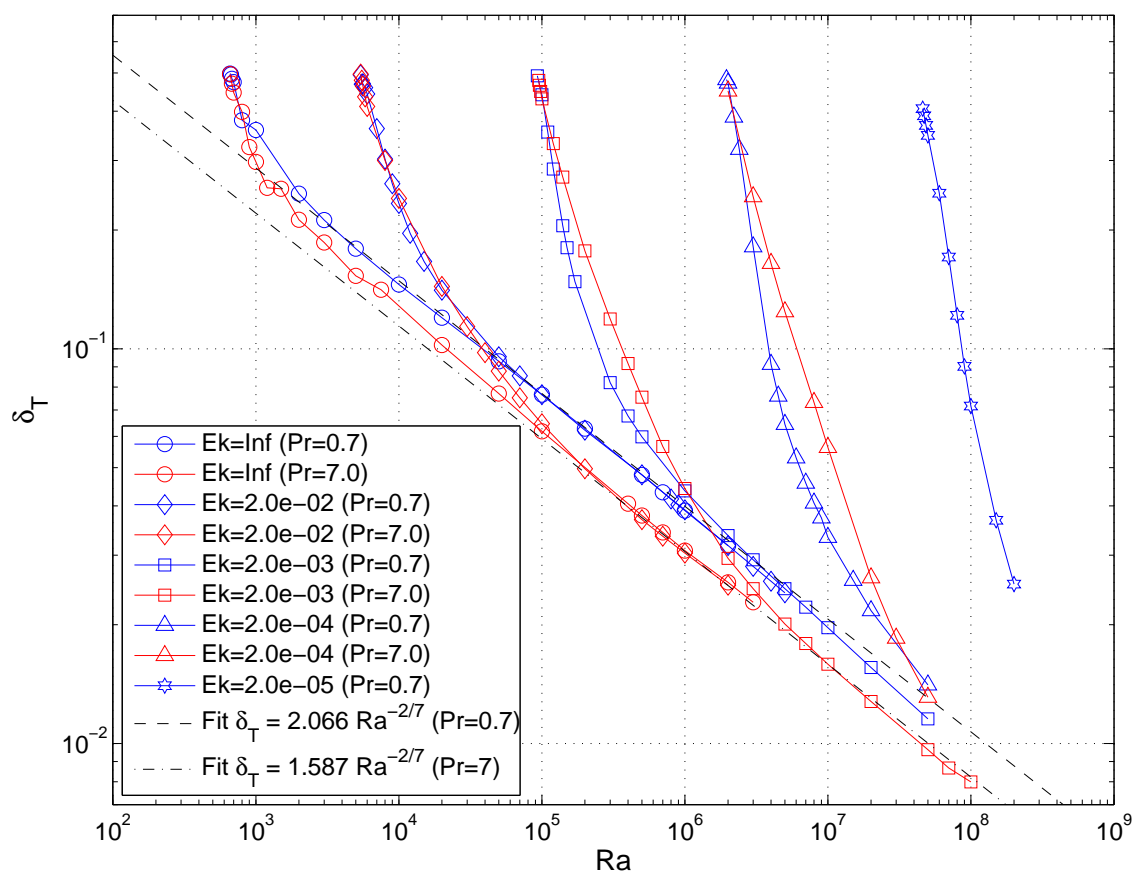

Abb. 5.15: Thermische Grenzschichtdicke $\delta_{T}$ über der Rayleigh-Zahl $R a$.

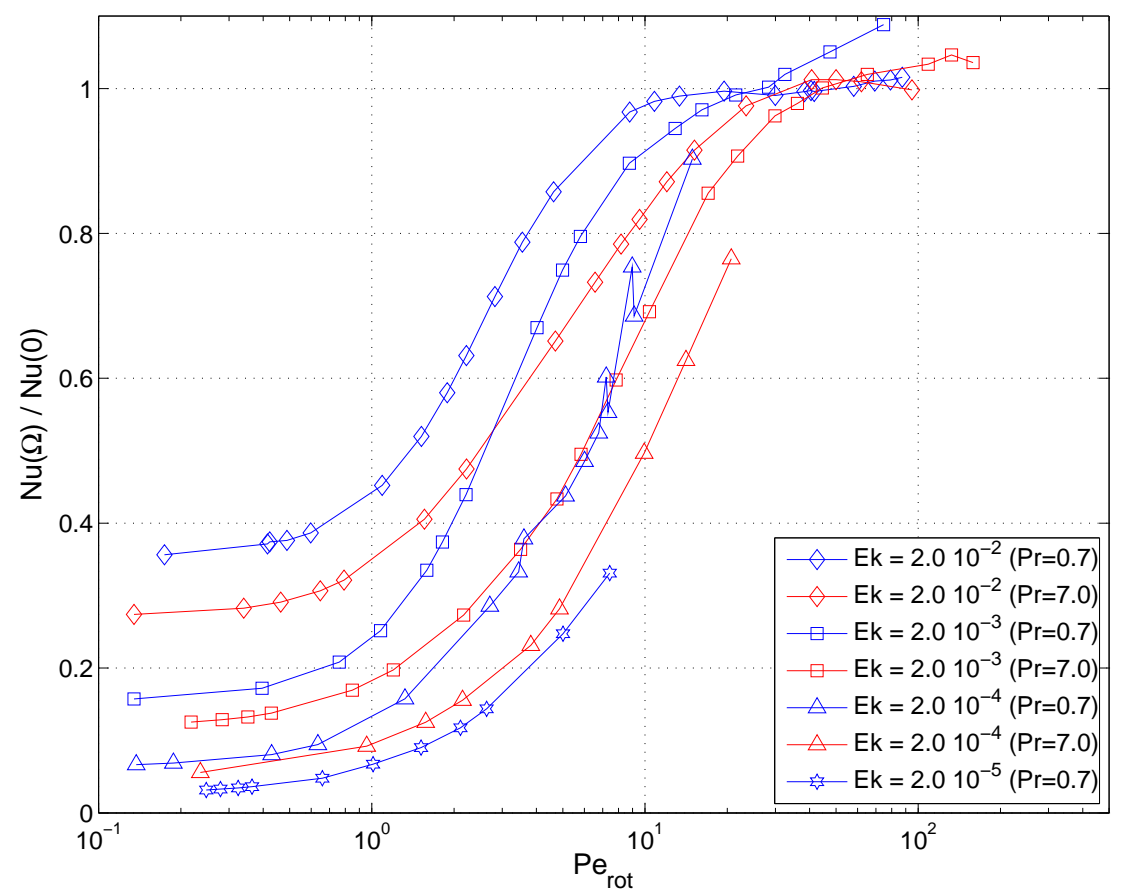

Abb. 5.16: Verhältnis $N u(\Omega) / N u(0)$ in Abhängigkeit der Rotations-Péclet-Zahl. Ein „Überschießen“ wie im no-slip Fall ist nicht zu erkennen. 

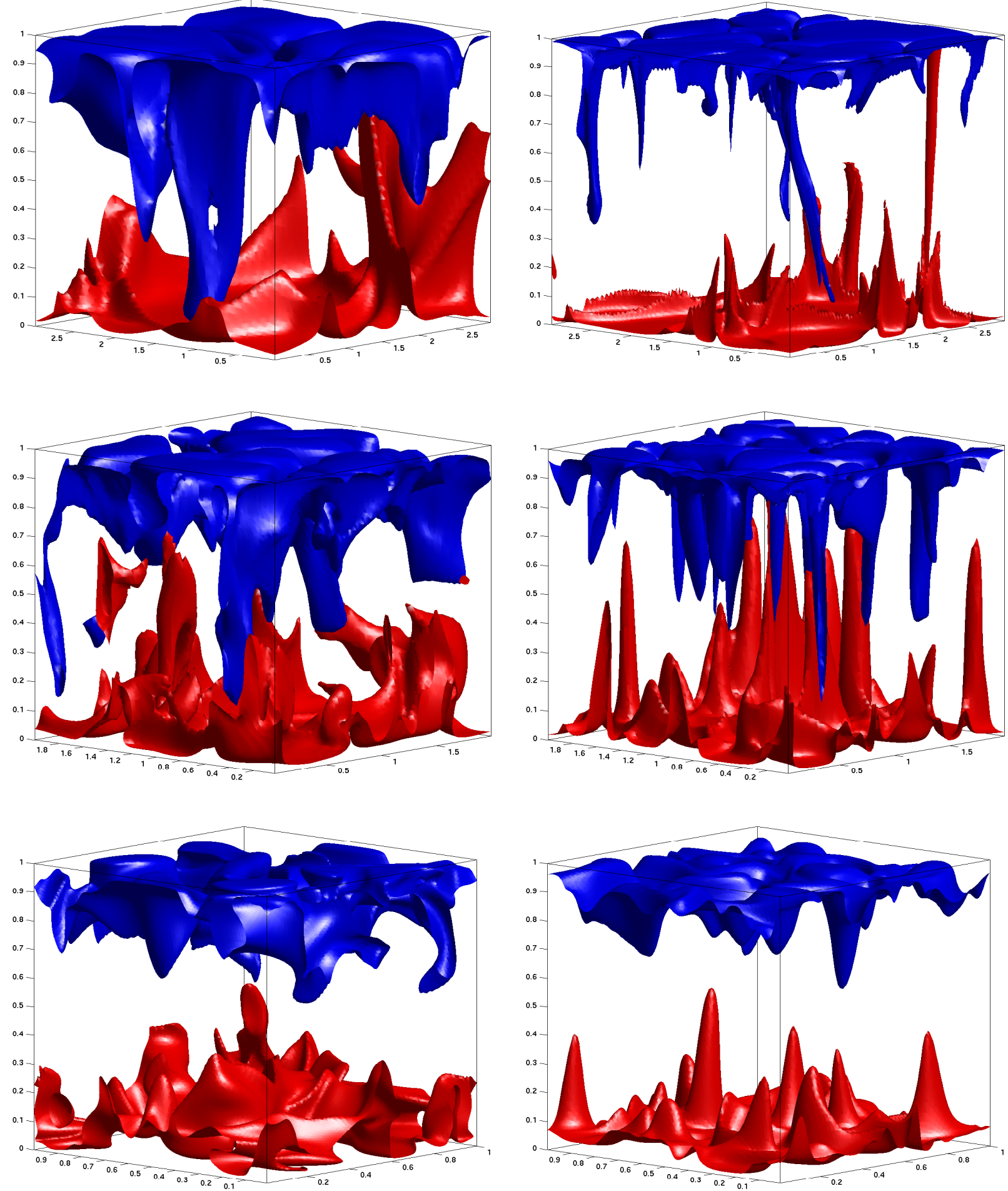

Abb. 5.17: Isoflächen der Temperatur für $\operatorname{Pr}=0.7$ (linke Spalte) und $\operatorname{Pr}=7.0$ (rechte Spalte). Oben: $E k=2 \cdot 10^{-2}, R a=1 \cdot 10^{5}, T=0.75 \Delta T$ (rot) und $T=0.25 \Delta T$ (blau). Mitte: $E k=2 \cdot 10^{-3}, R a=1 \cdot 10^{6}, T=0.7 \Delta T$ (rot) und $T=0.3 \Delta T$ (blau). Unten: $E k=2 \cdot 10^{-4}, R a=1 \cdot 10^{7}, T=0.65 \Delta T$ (rot) und $T=0.35 \Delta T$ (blau). 
Vorfaktoren, die man nach den Fits aus Abbildung 5.9 und Gleichung (5.7) erwartet. Wie man sieht, werden diese gut erfüllt.

Anzumerken ist, dass in den vorliegenden Simulationen mit schubspannungsfreien Rändern oben und unten kein „Überschießen“ der Nusselt-Zahl zu beobachten ist. In der Literatur (siehe [24; 41]) mit no-slip Rändern übersteigt die Nusselt-Zahl beim Übergang vom rotationsdominierten Regime zum rotationsfreien den Wert der Nusselt-Zahl ohne Rotation deutlich. Dies ist besonders in einer kompensierten Auftragung $N u(\Omega) / N u(0)$ gut zu sehen. Für dieses Verhalten sind Ekman-Pumpen in der Grenzschicht verantwortlich (siehe [26]).

In einer solchen Auftragung mit schubspannungsfreien Rändern in Abbildung 5.16 ist dieses Verhalten nicht zu sehen, wie auch in [25] gezeigt wurde. Dabei ist für $N u(0)$ der Fit aus Abbildung 5.9 für die jeweiligen Prandtl-Zahlen angesetzt worden. Lediglich für die Ergebnisse bei $E k=2 \cdot 10^{-3}$ erkennt man eine Überhöhung bei großen Werten von Pe $e_{\text {rot }}$. Diese hängt aber damit zusammen, dass dort (die entsprechenden Rayleigh-Zahlen liegen im Bereich $3 \cdot 10^{6} \leq R a \leq 1 \cdot 10^{8}$ ) für schubspannungsfreie Ränder $N u-1 \propto R a^{1 / 3}$ erwartet wird (siehe [23]).

Zum Schluss sollen noch die Temperaturfelder visualisiert werden. Deshalb sind in Abbildung 5.17 für beide Prandtl-Zahlen und drei Ekman-Zahlen die dreidimensionalen Temperaturisoflächen dargestellt. Dabei ist der Übersichtlichkeit wegen nicht immer das gesamte Strömungsfeld gezeigt, sondern nur ein Ausschnitt des Aspektverhältnisses.

Man erkennt eindeutig den Unterschied zwischen den beiden Prandtl-Zahlen: Für die kleine Prandtl-Zahl von $\operatorname{Pr}=0.7$ (links in Abbildung 5.17) sind die Strukturen breiter, weil die thermische Diffusion die kinetische Diffusion überwiegt. Für große Prandtl-Zahl von $\operatorname{Pr}=7.0$ ist es genau umgekehrt (rechts in Abbildung 5.17). Die vorherrschenden Strukturen sind lange, schmale Wirbel. Man erkennt außerdem an den Bildern, dass die auf- und absteigenden Strukturen in sich verdreht sind. Der Grund dafür wird in Abschnitt 5.4.1 bei der Besprechung der Strömungsstrukturen betrachtet.

\subsubsection{Diffusionsfreie Kennzahlen}

In diesem Abschnitt wird der Übergangsbereich $0.5 \leq P e_{\text {rot }} \leq 10$ näher untersucht. Dazu werden diffusionsfreie Kennzahlen eingeführt.

Es ist klar, dass die Strömung im rotationsdominierten Bereich nahe des Onsets gegeben ist als ein Gleichgewicht aus der Auftriebskraft auf der einen Seite und der Corioliskraft und Diffusion auf der anderen Seite. Für kleine Werte von $P e_{\text {rot }}$ ist die Diffusion also nicht zu 
Kapitel 5. Ergebnisse

vernachlässigen. Für große Werte von $P e_{\text {rot }}$ ist der Einfluss des Coriolisterms klein gegenüber dem nichtlinearen Term. Das Verhalten der Nusselt-Zahl entspricht dort dem rotationsfreien Fall, wie in Abschnitt 5.3.2 gezeigt. Der Wärmefluss ist durch die thermische Grenzschicht gegeben, durch die die gesamte Wärme diffundieren muss.

Die Annahme für den Transitionsbereich $\left(0.5 \leq P e_{\text {rot }} \leq 10\right)$ ist die, dass die Strömung durch den nichtlinearen Term auf der einen Seite und die Corioliskraft auf der anderen Seite bestimmt ist. Weiter kann man annehmen, dass der Wärmefluss nicht mehr durch die Diffusion in der Grenzschicht bestimmt ist, sondern durch die Struktur der Strömung. Für diese Annahmen ist es nützlich, diffusionsfreie Kennzahlen (siehe [12]) einzuführen. So wird aus der Rayleigh-Zahl durch geeignete Kombination der Ekman- und Prandtl-Zahlen eine diffusionsfreie RayleighZahl:

$$
R a_{*}=R a \frac{E k^{2}}{P r}=\frac{g \alpha \Delta T}{\Omega^{2} d}
$$

Dies ist die einzige Kombination, die möglich ist. Ebenso verfährt man mit der diffusionsfreien Nusselt-Zahl:

$$
N u_{*}=N u \frac{E k}{P r}=\frac{Q}{\varrho c_{p} \Delta T \Omega d}
$$

Es ist für manche Anwendungsfälle sinnvoll, neben der diffusionsfreien Rayleigh-Zahl noch eine (ebenfalls diffusionsfreie) Fluss-Rayleigh-Zahl zu definieren:

$$
R a_{f *}=R a_{*} N u_{*}=R a N u \frac{E k^{3}}{P r^{2}}=\frac{g \alpha Q}{\varrho c_{p} \Omega^{3} d^{2}}
$$

In astrophysikalischen Untersuchungen kann man teilweise leichter den Wärmefluss $Q$ als die Temperaturdifferenz $\Delta T$ messen. Allerdings ist $R a_{f *}$ keine a priori bekannte Größe für das System. Da sie die Nusselt-Zahl enthält, kann sie erst im Nachhinein berechnet werden.

Ein Potenzgesetzt, welches den Zusammenhang zwischen $N u_{*}$ und $R a_{f *}$ beschreibt, ist also:

$$
N u_{*} \propto\left(R a_{f *}\right)^{\eta} \Longrightarrow \frac{Q}{\varrho c_{p} \Delta T \Omega d} \propto\left(\frac{g \alpha Q}{\varrho c_{p} \Omega^{3} d^{2}}\right)^{\eta}
$$

Eine in (positive) z-Richtung orientierte Rotationsachse wirkt sich durch den Coriolisterm so aus, dass sie einen großen Einfluss auf die Strömung in einer Ebene senkrecht dazu hat, also in konstanter Höhe z. Die Rotation führt somit dazu, dass die Strömung genähert als zweidimensional betrachtet werden kann, die von der Höhe $d$ des Gebiets unbeeinflusst ist.

Unter diesen Annahmen erwartet man für die Abhängigkeit der diffusionsfreien Nusselt-Zahl von der diffusionsfreien Fluss-Rayleigh-Zahl nach Gleichung (5.8) gerade:

$$
N u_{*} \propto\left(R a_{f *}\right)^{1 / 2}
$$


Der Exponent $\eta=\frac{1}{2}$ führt dazu, dass die Gleichung von der Zellhöhe $d$ unabhängig ist. Die Auftragung in Abbildung 5.18 zeigt die Simulationen zu allen untersuchten Ekman-Zahlen, die im Transitionsbereich $\left(0.5 \leq P e_{\text {rot }} \leq 10\right)$ liegen.

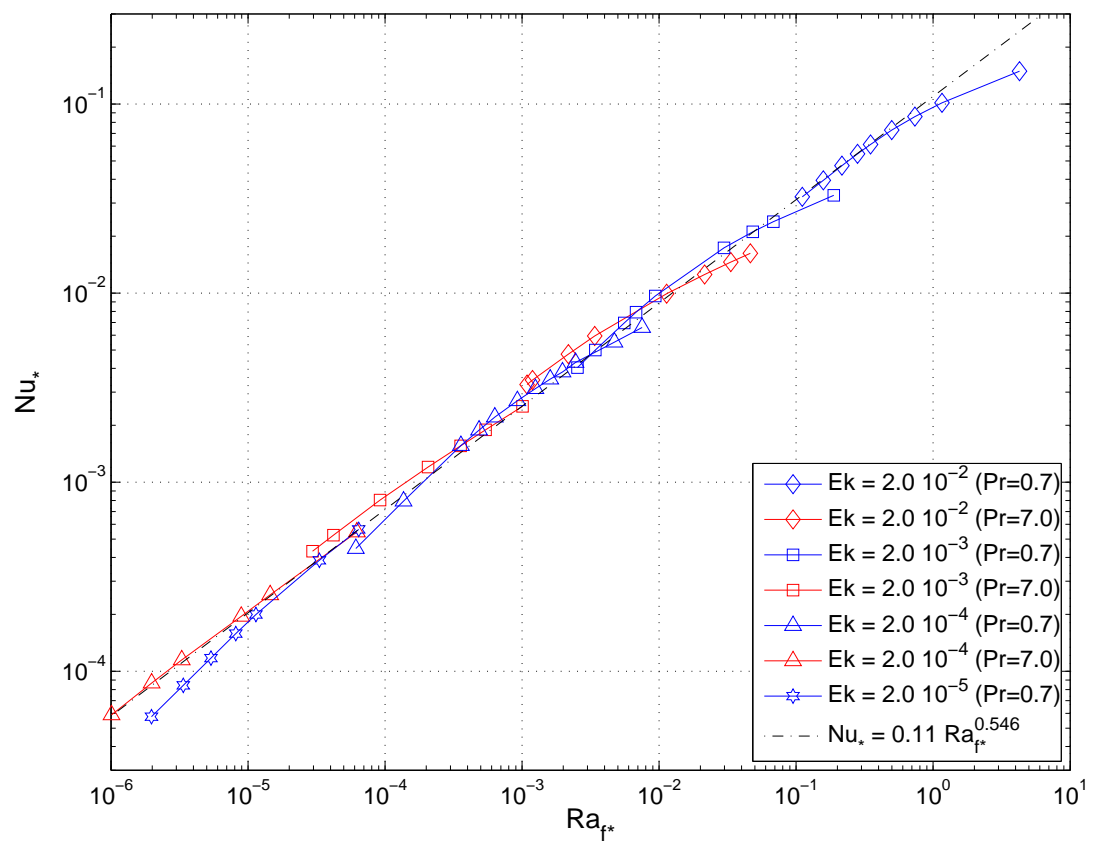

Abb. 5.18: Auftragung der diffusionsfreien Kennzahlen $N u_{*}$ über $R a_{f *}$ für alle Simulationen mit schubspannungsfreien Rändern aus dem Bereich $0.5 \leq P e_{\text {rot }} \leq 10$.

Der beste Fit über alle Simulationen liefert:

$$
N u_{*}=0.11\left(R a_{f *}\right)^{0.546( \pm 0.01)}
$$

Der Exponent ist zwar nicht $\frac{1}{2}$ wie angenommen, aber sehr nahe an $\frac{5}{9} \approx 0.556$. Diesen Wert ergeben z.B. auch Simulationen aus [12]. Aber auch ein Exponent von $\frac{6}{11} \approx 0.546$ wäre logisch. Durch Umformen von Gleichung (5.5) (mit $\mu$ als variablem Exponenten) und Gleichung (5.8) erhält man folgenden Zusammenhang

$$
\begin{aligned}
N u & =\left(\frac{R a}{R a_{c}}\right)^{\mu}, \quad N u_{*} \propto\left(R a_{f *}\right)^{\eta} \\
\mu & =\frac{\eta}{1-\eta} \quad \Longleftrightarrow \quad \eta=\frac{\mu}{1+\mu}
\end{aligned}
$$

Ist also die Steigung am Onset, wie oben schon geschrieben und in Abbidung 5.9 eingezeichnet, gerade $\mu=\frac{6}{5}$, so folgt daraus für die diffusionsfreien Kennzahlen ein Exponent von $\eta=\frac{6}{11}$. Natürlich ist der Exponent $\frac{6}{5}$ nur eine Näherung. Für die Simulationen mit $\operatorname{Pr}=7.0$ passt sie allerdings gut, für $P r=0.7$ müsste er etwas größer sein. 


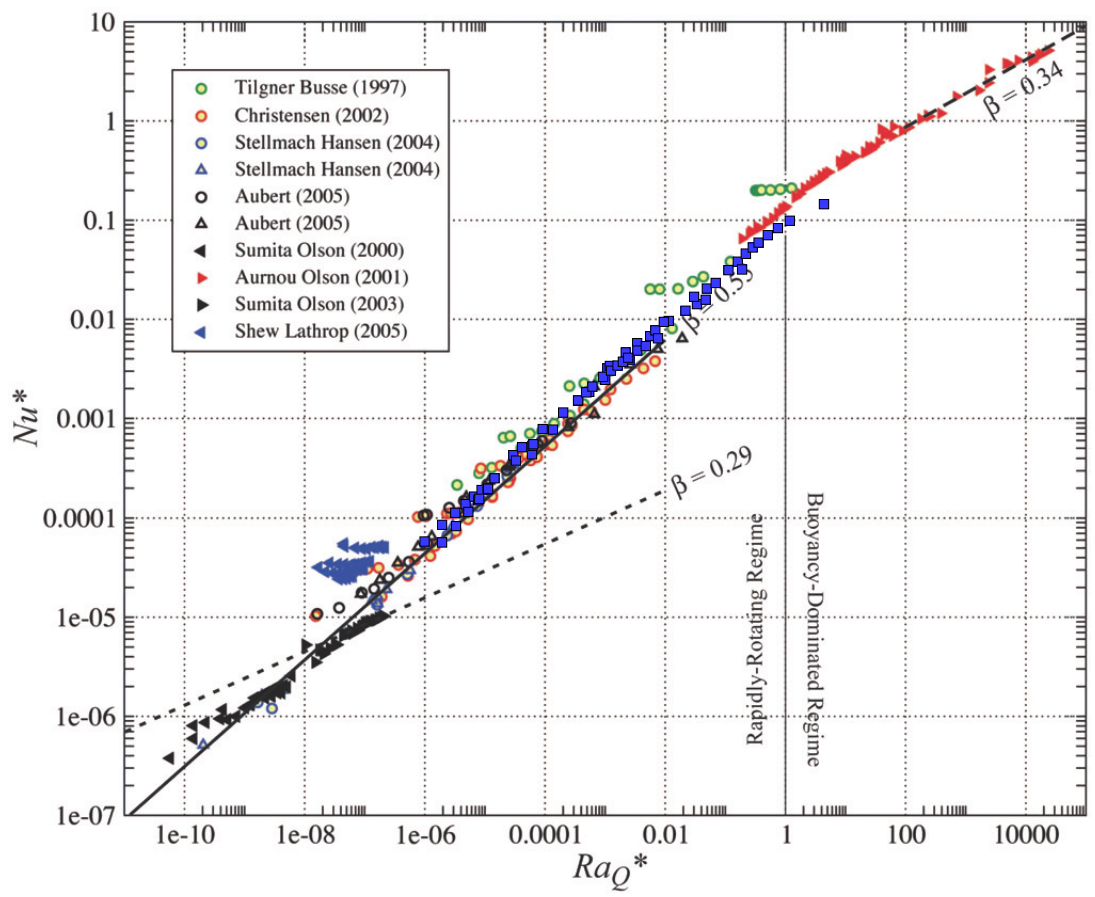

Abb. 5.19: Vergleich der diffusionsfreien Kennzahlen dieser Arbeit (blaue Quadrate) mit den Ergebnissen anderer Veröffentlichungen (Original entnommen aus [5]).

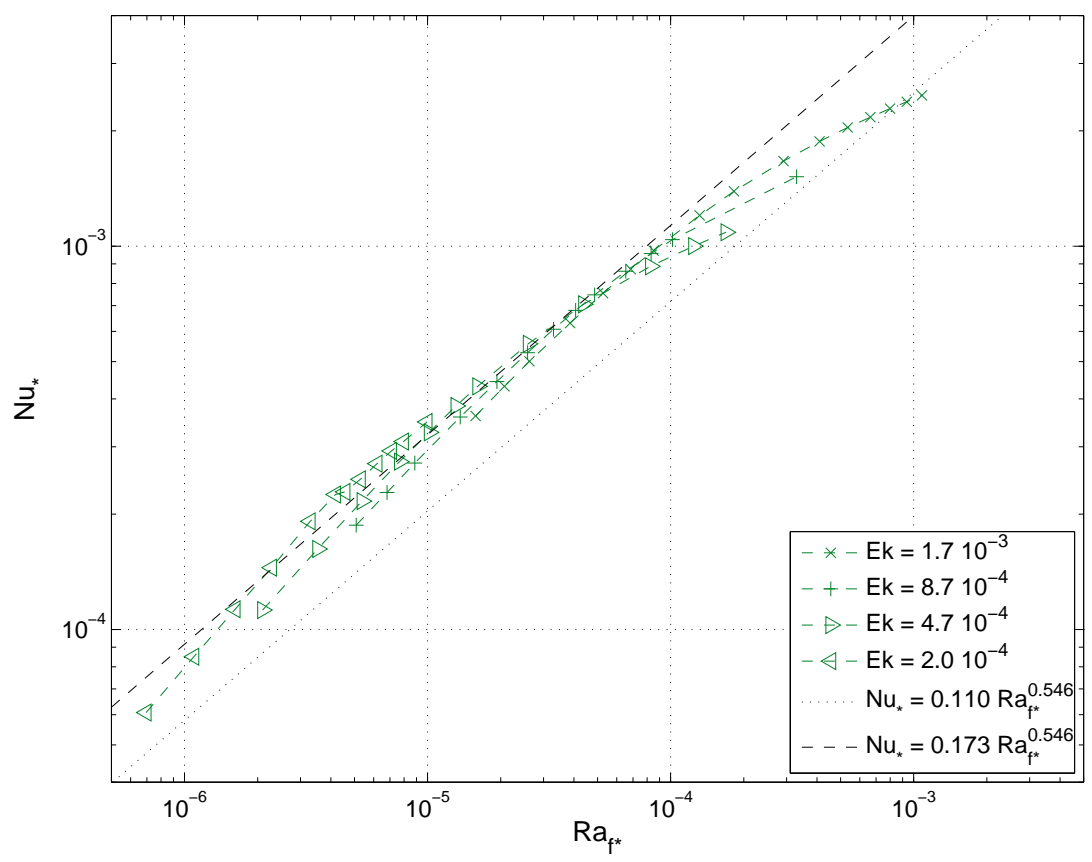

Abb. 5.20: Auftragung der diffusionsfreien Kennzahlen $N u_{*}$ über $R a_{f *}$ für alle Simulationen mit no-slip Rändern aus dem Bereich $0.5 \leq P e_{\text {rot }} \leq 10$. 
Einen Exponent von 0.55 liefern auch verschiedene numerische Simulationen und Experimente, zusammengestellt in [5]. Bemerkenswert ist dabei, dass sowohl planare Geometrien mit vertikal gerichteter als auch Kugelgeometrien mit radialer Schwerebeschleunigung und sowohl feste als auch schubspannungsfreie Wände das gleiche Ergebnis liefern. Abbildung 5.19 ist aus [5] entnommen und ergänzt durch die Ergebnisse der vorliegenden Arbeit, die als blaue Quadrate dargestellt sind. Man erkennt, dass die Ergebnisse der Arbeit gut in das Gesamtbild passen. Dies trifft natürlich umso besser für $R a_{f *} \ll 1 \mathrm{zu}$. Nur dort dominiert die Corioliskraft über die Auftriebskraft. Für Werte von $R a_{f *} \approx 1$ oder größer ist es genau umgekehrt. Man erwartet dann, dass die Corioliskraft keine Rolle mehr spielt. Dies drückt sich in Gleichung (5.8) gerade dadurch aus, dass sie unabhängig von der Ekman-Zahl sein sollte. Das führt zu einem Exponenten von $\eta=\frac{1}{3}$, wie in Abbildung $5.19 \mathrm{zu}$ sehen.

Der Fall für die Simulationen mit no-slip Rändern zeigt in Abbildung 5.20 ein ähnliches Ergebnis. Der Exponent $\eta=0.546$ ist der gleiche wie im Fall mit schubspannungsfreien Rändern. Lediglich der Vorfaktor ist verschieden (zum Vergleich ist nochmal die Fitgerade wie in Abbildung 5.18 gepunktet mit eingezeichnet).

\subsection{Strömungsstrukturen}

In diesem Abschnitt wird die Größe der Strömungsstrukturen untersucht. Dazu wird die normierte Helizität der Strömung berechnet. Sie dient als Maß dafür, inwieweit die Strömung noch durch die Corioliskraft beeinflusst wird. Zum Schluss werden noch räumliche Spektren des konvektiven Wärmetransports über die Zeit gemittelt und über der Wellenlänge $\lambda$ aufgetragen, um die Strukturen auch quantitativ beurteilen zu können.

\subsubsection{Helizität der Strömung}

In diesem Abschnitt wird die Helizität der Strömung betrachtet (siehe z.B. [38]). Die Helizität ist der „Schraubsinn“ eines Fluidpartikels. Sie ist gegeben durch das Skalarprodukt aus der Winkelgeschwindigkeit $\boldsymbol{\omega}=\nabla \times \mathbf{u}$ und der Geschwindigkeit $\mathbf{u}$. Im vorliegenden Fall interessiert die normierte Helizität $H$ :

$$
H=\frac{\boldsymbol{\omega} \cdot \mathbf{u}}{|\boldsymbol{\omega}| \cdot|\mathbf{u}|}
$$

Ein Fluidteilchen kann sich in Bezug auf seine Bewegungsrichtung im Uhrzeigersinn (positive Helizität, Rechtsschraubung) oder entgegen dem Uhrzeigersinn (negative Helizität, Linksschraubung) drehen. 

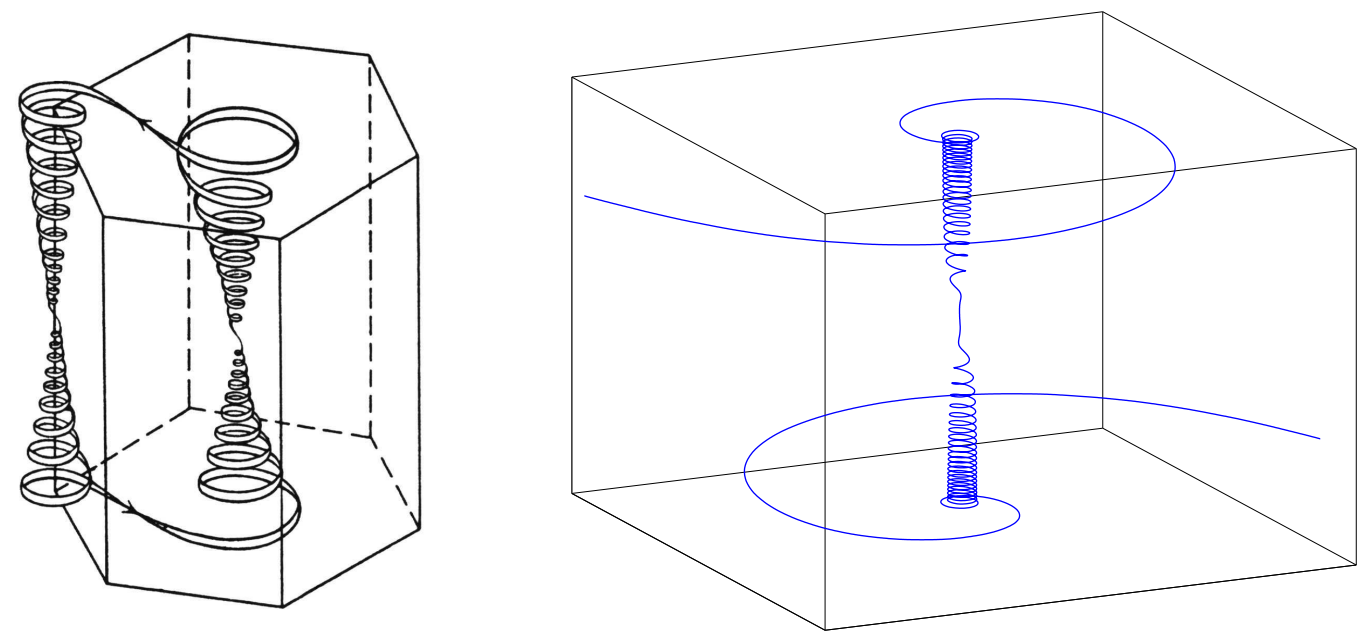

Abb. 5.21: Schraubenbewegung der Fluidteilchen unter dem Einfluss der Corioliskraft: Beispiel einer Teilchenbahn aus der Literatur (links, siehe [10]) und aus einer Simulation (rechts).

Bei rotierenden Konvektionsströmungen führt die Corioliskraft dazu, dass die Teilchen einer Konvektionsrolle beeinflusst werden. Die im vorliegenden Fall in positive z-Richtung orientierte Corioliskraft wirkt natürlich nicht auf Teilchen, die sich nur in (positive oder negative) $z$-Richtung bewegen. Alle anderen Teilchen werden abgelenkt, so dass sich schraubenförmige Bahnlinien einstellen. Dies ist in Abbildung $5.21 \mathrm{zu}$ sehen. Die Teilchen haben bei der hier gewählten Richtung der Corioliskraft immer positive Helizität in der unteren und negative in der oberen Hälfte des Simulationsgebiets. In der Mittelebene ist sie Null.

$\mathrm{Zu}$ jeder Rechnung wurde für einen Zeitpunkt im gesamten Strömungsgebiet die normierte Helizität berechnet. In Abbildung 5.22 ist für $E k=2 \cdot 10^{-3}$ und $\operatorname{Pr}=7.0$ das Ebenenmittel $\langle H\rangle_{x, y}$ dargestellt.

Natürlich sollte das Ebenenmittel bei $z=0.5$ Null sein. Da es sich bei der Auftragung aber um Werte handelt, die aus dem Strömungsfeld eines Zeitpunkts berechnet wurden, also keine zeitlichen Mittel sind, kommt es zu einer kleinen Abweichung. An den Rändern bei $z=0$ und $z=1$ ist die Helizität aufgrund der geforderten Randbedingungen nach Gleichung (2.2a-2.2c) Null. Mittelt man über das Volumen der gesamten Zelle, so ist das Ergebnis Null.

Das Maximum von $\langle H\rangle_{x, y}$ ist abhängig von der Rayleigh-Zahl. Mit steigender Rayleigh-Zahl verlagert es sich weiter zum Rand, ähnlich wie bei den Grenzschichten aus Abschnitt 5.1. Damit einhergehend wird es immer kleiner, die Strömung verliert ihre helikale Struktur. Wenn die Corioliskraft (bei genügend großen Rayleigh-Zahlen) schließlich keine Rolle mehr spielt, dann ist kein Maximum des Ebenenmittels der Helizität $H$ mehr erkennbar, es schwankt um den Nullwert. 


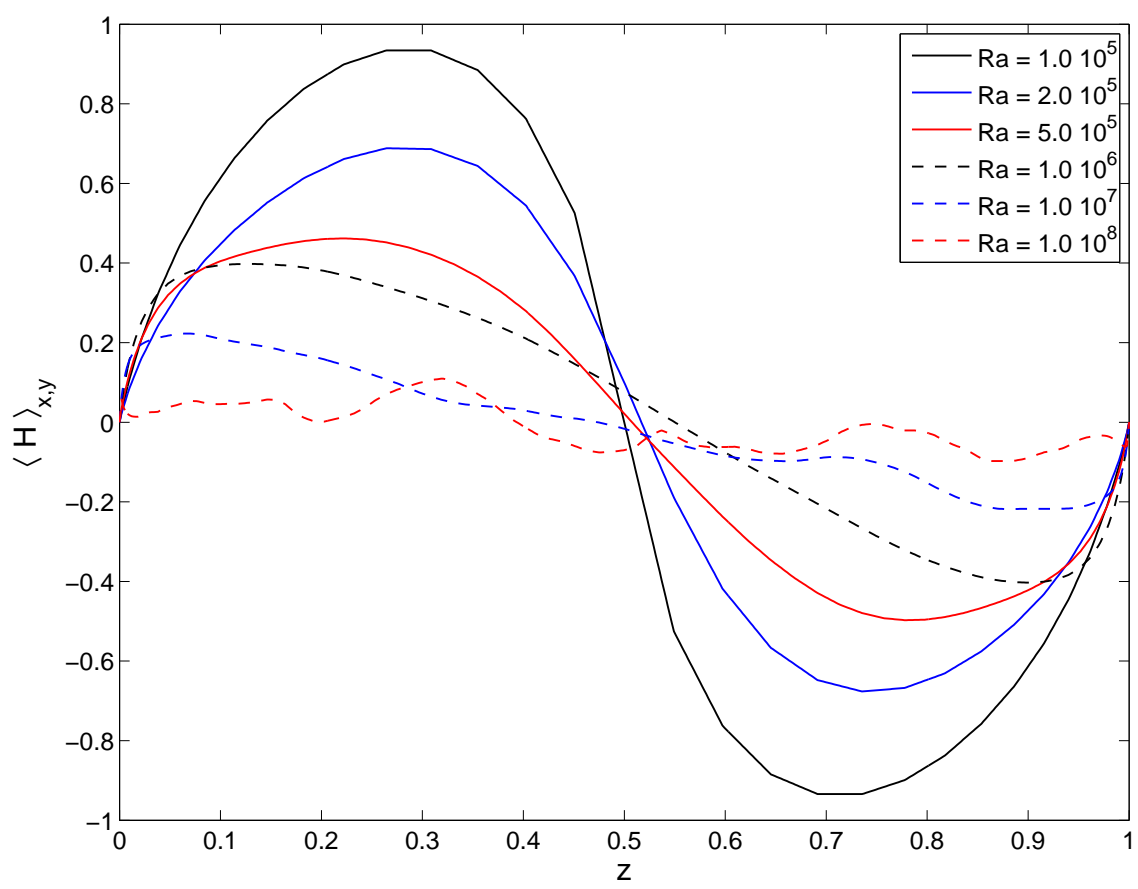

Abb. 5.22: Ebenenmittel der Helizität aufgetragen über der Zellhöhe für sechs verschiedene RayleighZahlen bei konstanter Ekman- und Prandtl-Zahl $\left(E k=2 \cdot 10^{-3}, \operatorname{Pr}=7.0\right)$.

Um nun die Größe der Helizität in der Strömung zu bestimmen, wird der rms-Wert eines jeden Ebenenmittels berechnet:

$$
H_{r m s}=\left\langle\langle H\rangle_{x, y}\right\rangle_{r m s}
$$

Die Werte für den Fall ohne Rotation sind nicht explizit gezeigt, da dort der rms-Wert klein ist. Er liegt im Mittel bei $H_{r m s} \approx 0.02$. In Abbildung 5.23 (links) ist die Größe $H_{r m s}$ für alle Simulationen mit Rotation dargestellt. Sie ist, wie schon bei der Betrachtung des Wärmetransports, über der Rotations-Péclet-Zahl aufgetragen.

Der auffälligste Unterschied zu den Abbildungen aus Abschnitt 5.3.2 ist, dass die Kurven nicht mehr zusammenfallen. Es ist eindeutig zu erkennen, dass die Werte bei der großen PrandtlZahl weiter nach rechts verschoben sind. Die Kurven kommen erst in einer Auftragung über der Rotations-Reynolds-Zahl $R e_{\text {rot }}=R e E k^{1 / 2}$ übereinander zu liegen, siehe Abbildung 5.23 (rechts).

Möchte man eine Schwelle definieren, ab der die Strömung nicht mehr von der Rotation bestimmt wird, so ist die normierte Helizität eine sinnvolle Wahl. Sinkt nämlich $H_{r m s}$ ab bis auf einen Wert, den man auch in nichtrotierenden Strömungen findet, so spielt die Corioliskraft keine Rolle mehr. In Abbildung 5.23 sind jeweils bei $H_{r m s}=0.02$ Linien eingetragen, um die 
Schwelle leichter zu identifizieren. Diese liegt bei $R e_{\text {rot }} \approx 50$.
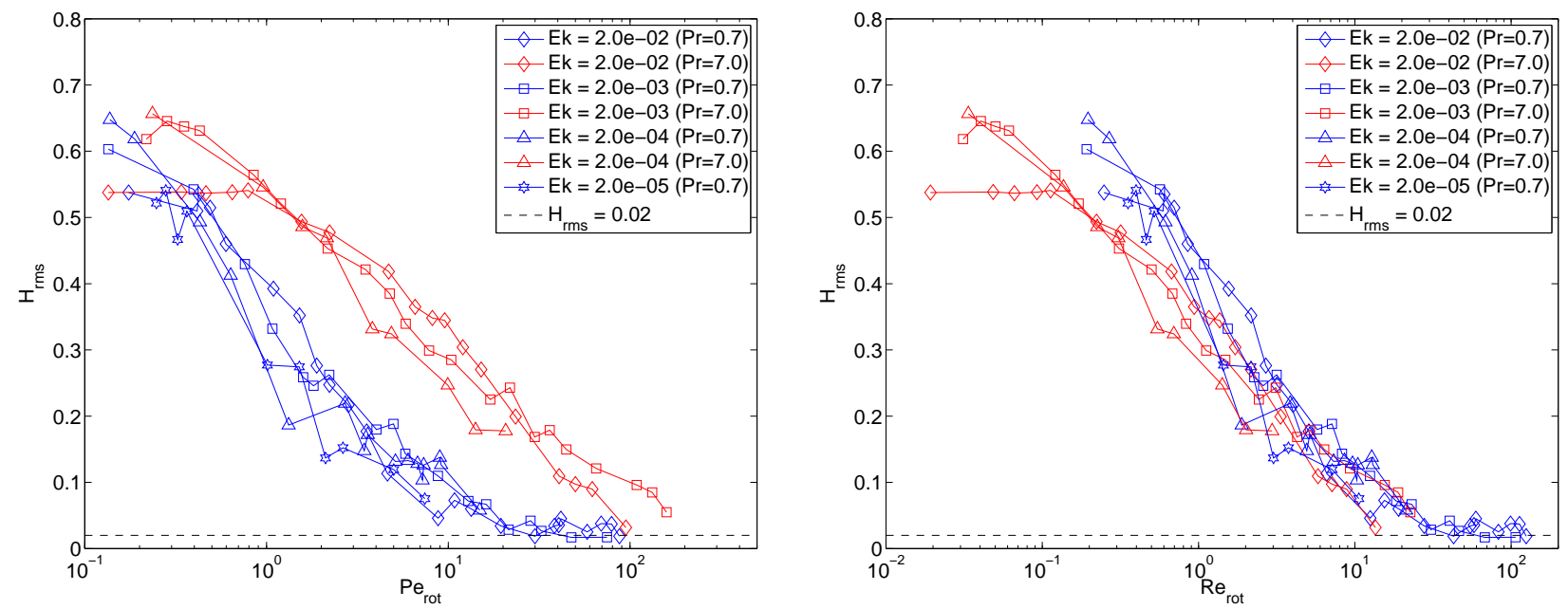

Abb. 5.23: Auftragung des rms-Werts der Helizität $H_{r m s}$ über $P e_{\text {rot }}$ (links) und $R e_{\text {rot }}$ (rechts) für Simulationen mit Rotation.

Um dieses Ergebnis zu untermauern, wird nun die kinetische Energiedissipation betrachtet. Sie wird automatisch im Programm in den Messroutinen ausgerechnet. In Abbildung 5.24 ist sie für Simulationen ohne Rotation aufgetragen über der Reynolds-Zahl, normiert mit $\operatorname{Re}^{3} \operatorname{Pr}^{2}$, wie man es im turbulenten Fall bei großen Rayleigh- bzw. Reynolds-Zahlen erwartet (siehe [18]). Bei hohen Reynolds-Zahlen erkennt man auch einen Übergang der Kurven zu einem konstanten Wert, der bei $\epsilon_{u} \approx 0.3$ liegt. Bei kleinen Reynolds-Zahlen sollte sich die kinetische Energiedissipation proportional zu $R e^{2}$ verhalten. Auch dies sieht man im Graphen, der dort gerade eine Steigung von -1 aufweist.

Abbildung 5.25 zeigt die gleiche normierte kinetische Energiedissipation für Simulationen mit Rotation. Auf der Abszisse wird, wie auch schon bei der Helizität, die Größe $R e_{\text {rot }}=R e E k^{1 / 2}$ verwendet. Eine Linie bei $\epsilon_{u} /\left(\operatorname{Re}^{3} \operatorname{Pr}^{2}\right)=0.3$ soll die Identifizierung des Übergangs der Strömung zu einem rotationsfreien Zustand erleichtern. Man erkennt in der Abbildung, dass der gesuchte Übergang bei $R e_{\text {rot }} \approx 50$ liegt. Die Strömung verhält sich von da an so, als ob es keine Corioliskraft mehr gäbe. Die Schwelle ist unabhängig von der Prandtl-Zahl, sie hängt nur noch von der Reynolds- und Ekman-Zahl ab. Sie ist zudem identisch, sowohl für die Helizität als auch für die kinetische Energiedissipation.

\subsubsection{Spektren des konvektiven Wärmetransports}

Nun werden die Spektren des konvektiven Wärmetransports untersucht. Es wird also der konvektive Anteil $u_{z}(x, y, z) \cdot T(x, y, z)$ des Wärmestroms für jede horizontale Ebene $z$ in spektraler 


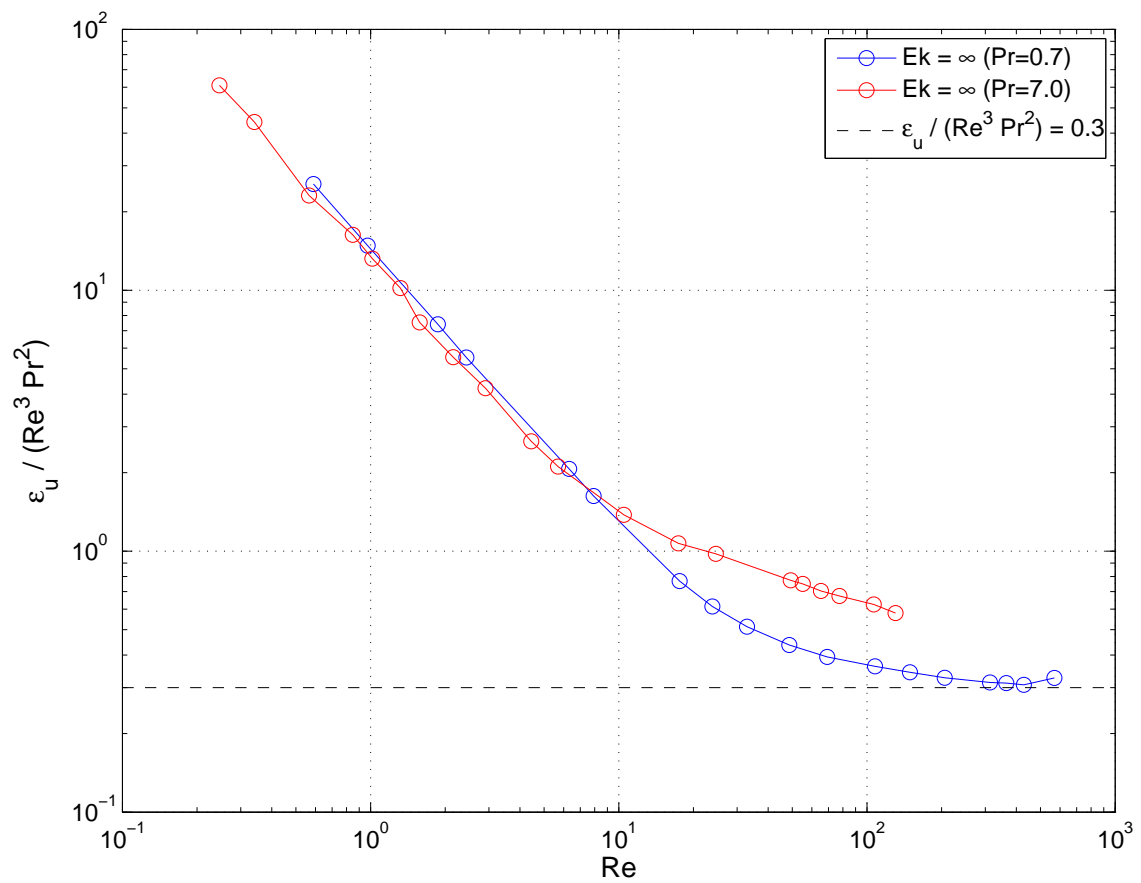

Abb. 5.24: Kinetische Energiedissipation $\epsilon_{u}$ für Simulationen ohne Rotation, normiert mit $\operatorname{Re}^{3} \operatorname{Pr}^{2}$, aufgetragen über der Reynolds-Zahl Re.

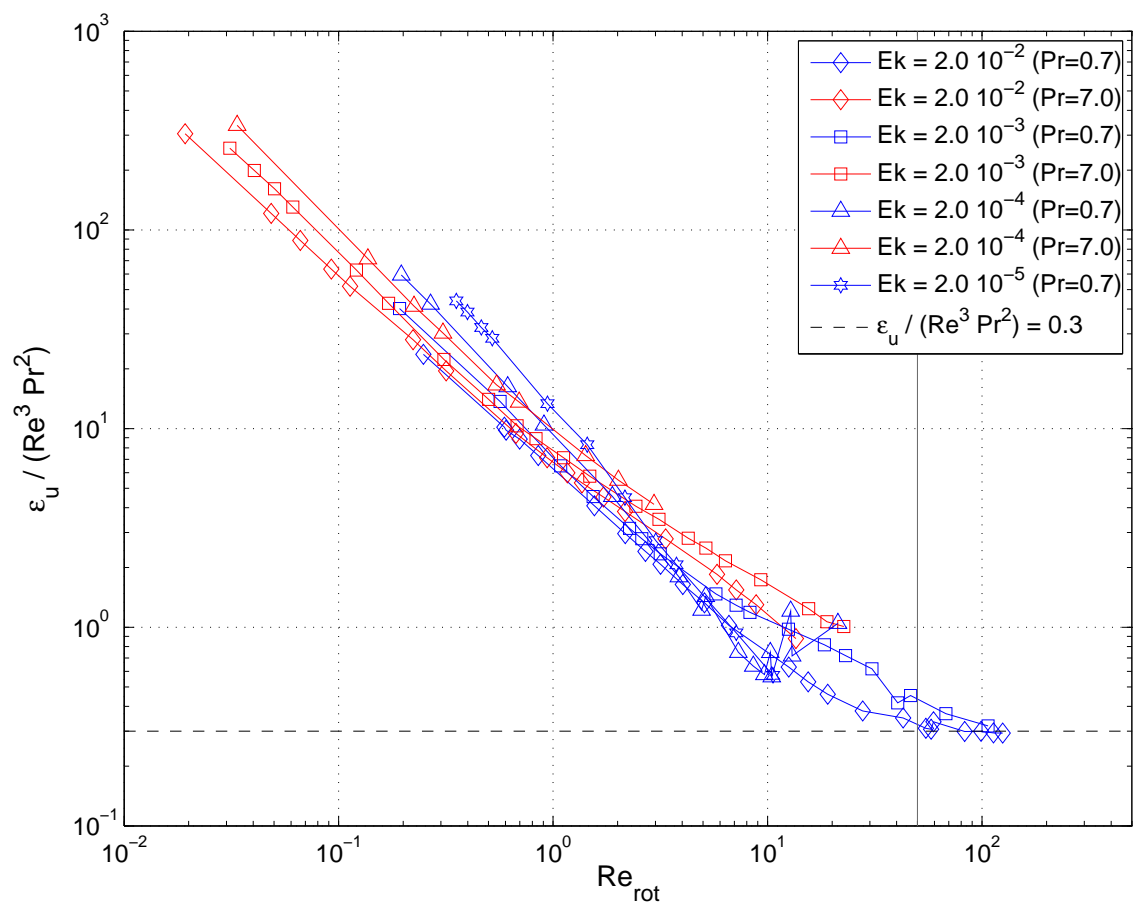

Abb. 5.25: Kinetische Energiedissipation $\epsilon_{u}$ für Simulationen mit Rotation, normiert mit dem rotationsfreien Verhalten $\operatorname{Re}^{3} \mathrm{Pr}^{2}$, aufgetragen über der Rotations-Reynolds-Zahl $R e_{\text {rot }}$. 
Form berechnet, siehe Gleichung (2.5). Dies ist nicht schwer, da im Programm die Variablen sowieso im Spektralraum vorliegen. Es ergibt sich für die spektrale Verteilung $V$;

$$
V(k, z)=\frac{1}{N u-1} \sum_{\mathbf{k} \text { mit }|\mathbf{k}|=k}\left(u_{z}(\mathbf{k}, z) \cdot T(\mathbf{k}, z)\right)
$$

Diese ist normiert auf den Wert des konvektiven Wärmetransports $(N u-1)$. Es ist zu beachten, dass es zu einer Wellenzahl $k=|\mathbf{k}|=\sqrt{k_{x}^{2}+k_{y}^{2}}$ durchaus mehrere Wellenvektoren $\mathbf{k}$ geben kann. Der Verlauf von $V(k, z)$ ist für $z=0.5$ exemplarisch in Abbildung 5.26 (links) gezeigt. Allerdings ist die Wellenzahl $k$ nicht äquidistant verteilt, bei kleinen $k$ gibt es weniger diskrete Werte als bei großen. Deshalb kann man nicht einfach ablesen, welcher Bereich der Wellenlänge hauptsächlich zum Wärmetransport beiträgt.

Es ist deshalb sinnvoller, die spektrale Dichte $D(\lambda, z)$ zu berechnen. Dazu wird der Bereich der möglichen Wellenlängen $0 \leq \lambda \leq L$ in äquidistante Intervalle $\Delta \lambda$ unterteilt. In diesen Intervallen wird der jeweilige Beitrag aufaddiert und durch die Intervallbreite dividiert:

$$
D(\lambda, z)=\frac{1}{N u-1} \sum_{\mathbf{k} \text { mit }} \sum_{\frac{2 \pi}{k} \in\left[\lambda_{i} ; \lambda_{i}+\Delta \lambda\right]} \frac{u_{z}(\mathbf{k}, z) \cdot T(\mathbf{k}, z)}{\Delta \lambda}
$$

Die spektrale Dichte ist auch normiert auf den Wert des konvektiven Wärmetransports $(N u-1)$. In Abbildung 5.26 (rechts) ist $D(\lambda, z)$ (mit $z=0.5$ ) ebenfalls gezeigt.
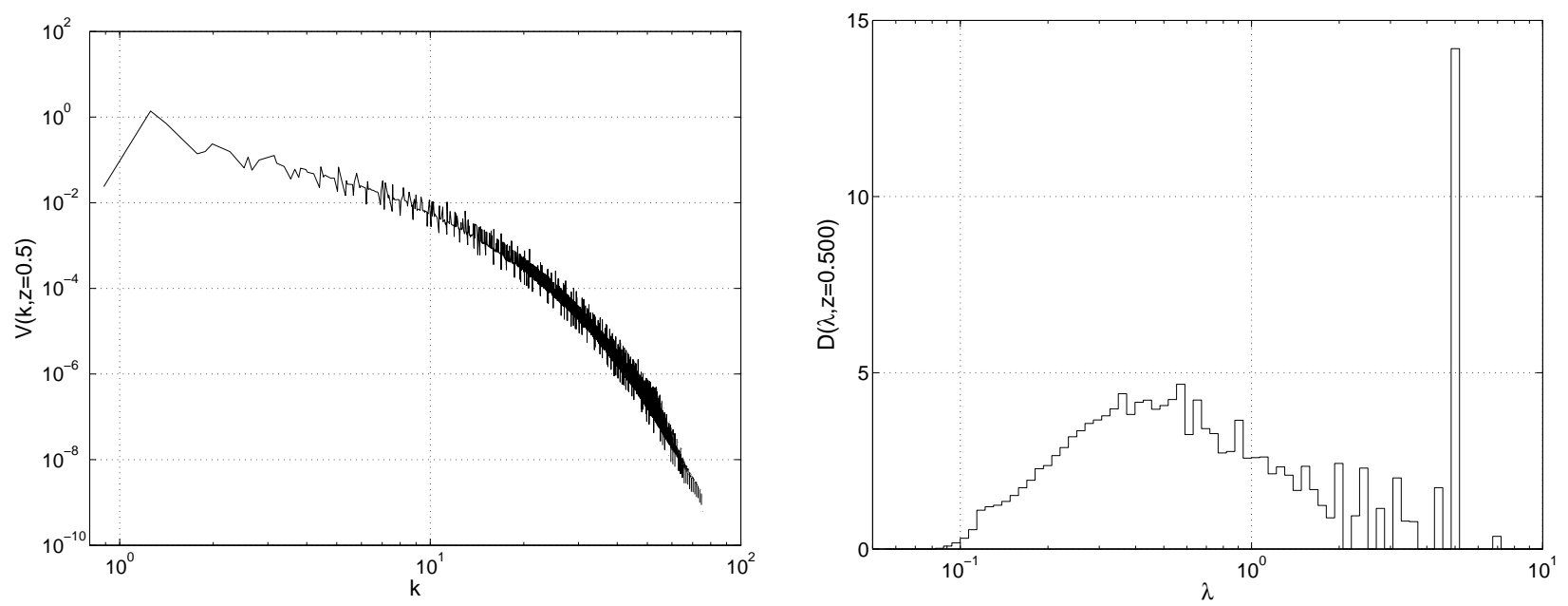

Abb. 5.26: Spektren des konvektiven Anteils $u_{z} T$ des Wärmestroms in der Mittelebene bei $z=0.5$ (links: spektrale Verteilung $V(k, z)$, rechts: spektrale Dichte $D(\lambda, z)$ ) einer Simulation mit $R a=1 \cdot 10^{6}, \operatorname{Pr}=0.7$ und $E k=\infty$.

Die dargestellten Spektren sind das Ergebnis einer rotationsfreien Simulation mit den Parametern $R a=1 \cdot 10^{6}, \operatorname{Pr}=0.7$ und $E k=\infty$. Man erkennt sehr gut, dass sie zweigeteilt sind. Es 
gibt Anteile mit großer Wellenlänge, die von den großskaligen Konvektionszellen herrühren. Die Strömung bei $z=0.5$ (also in der Zellmitte) wird natürlich hauptsächlich durch diese Rollen bestimmt. Daneben gibt es Anteile mit kleineren Wellenlängen, die kleinskalige Strukturen also z.B. Plumes oder Spokes - repräsentieren.

Trägt man zusätzlich noch Spektren näher an der Wand auf, so geht der Anteil der langwelligen Strukturen zurück. Der Wärmetransport in Wandnähe ist hauptsächlich durch Diffusion und Plumes bestimmt. Dies erkennt man gut in Abbildung 5.27, in der Spektren bei $z=0.164$ bzw. $z=0.015$ dargestellt sind.
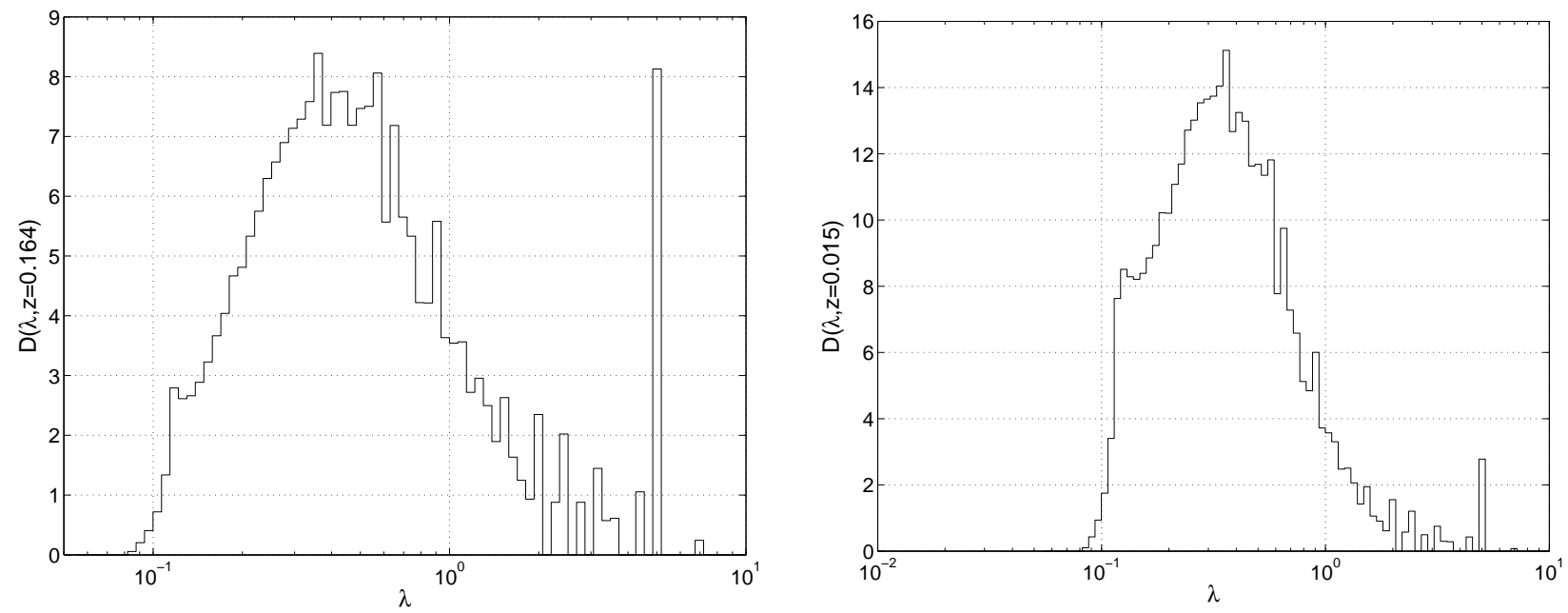

Abb. 5.27: Spektrale Dichte des konvektiven Wärmetransports $u_{z} T$, analog zu Abbildung 5.26. Die Schnitte sind hier bei den Höhen $z=0.164$ (links) und $z=0.015$ (rechts).

Um dem Spektrum objektiv eine repräsentative Wellenlänge zuordnen zu können, wurde ein Fit angepasst. Dieser ist in Abbildung 5.28 eingetragen. Die Fitkurve $F(\lambda)$ setzt sich dabei aus zwei halben, modifizierten Gaussglocken zusammen, eine linkseitige und eine rechtsseitige relativ zur gefitteten (gesuchten) Wellenlänge.

$$
F(\lambda)= \begin{cases}\frac{h}{\sqrt{2 \pi}} e^{-\frac{1}{2}\left(\frac{\lambda-\mu}{\sigma_{l}}\right)^{2}} & \lambda \leq \mu \\ \frac{h}{\sqrt{2 \pi}} e^{-\frac{1}{2}\left(\frac{\lambda-\mu}{\sigma_{r}}\right)^{2}} & \lambda \geq \mu\end{cases}
$$

Modifiziert bedeutet, dass das Integral nicht mehr auf 1 normiert ist, wie es bei einer Normalverteilung der Fall ist, bei der die Höhe und Breite der Verteilung miteinander verknüpft sind über

$$
N(\mu, \sigma)=\frac{1}{\sigma \sqrt{2 \pi}} e^{-\frac{1}{2}\left(\frac{x-\mu}{\sigma}\right)^{2}}
$$




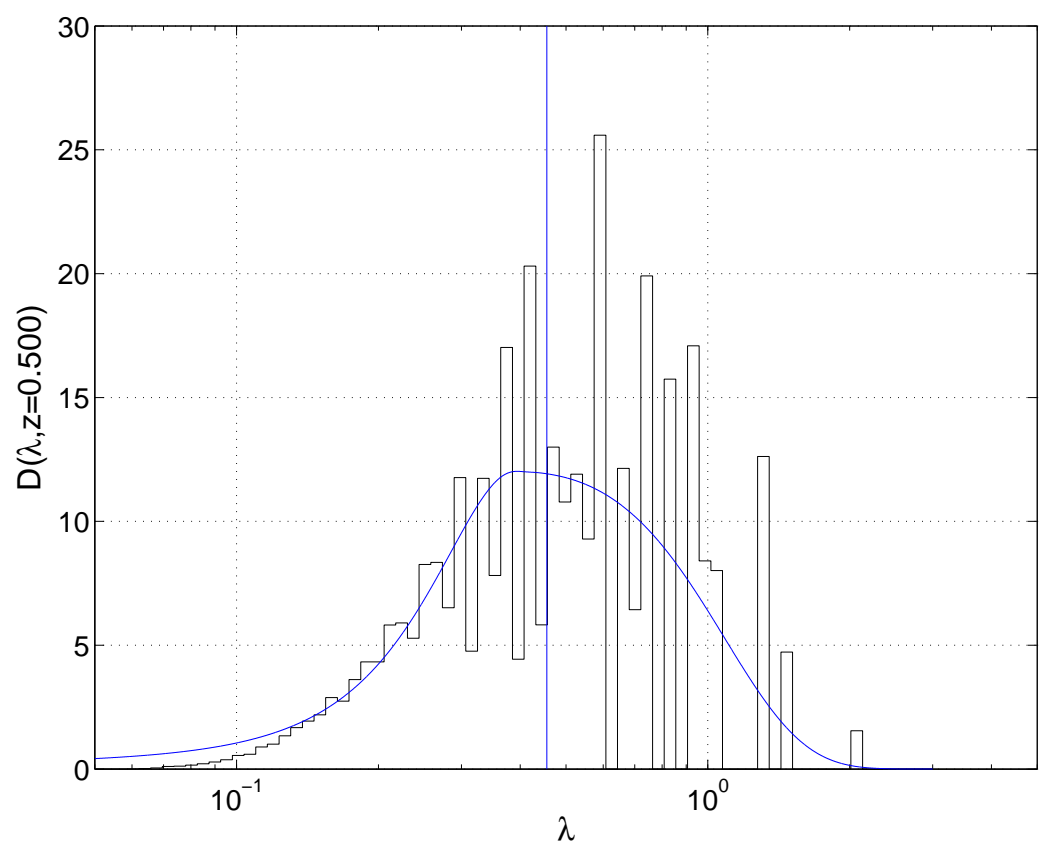

Abb. 5.28: Fit an die spektralen Dichte mithilfe einer Gaussglocke nach Gleichung (5.11). Eingezeichnet ist zusätzlich die gesuchte Wellenlänge $\lambda_{m}$.

Auf diese Weise erhält man vier Fitparameter, nämlich die Höhe $h$ und Position $\mu$ der Gaussglocke, die jeweils für beide Hälften natürlich identisch sein müssen, sowie die linksseitige $\sigma_{l}$ und rechtsseitige $\sigma_{r}$ Standardabweichung. Die Parameter wurden so bestimmt, dass die quadratische Abweichung minimal werden sollte. Dazu wurde der Simplex-Algorithmus nach Nelder und Mead (siehe [27], [30]) in einer Matlab-Implementation verwendet. Die gesuchte repräsentative Wellenlänge ist dann einfach das $\lambda_{m}$, das das Integral der Fitkurve halbiert:

$$
\int_{-\infty}^{\lambda_{m}} F(\lambda) d \lambda=0.5
$$

Es ist $\lambda_{m}$ sozusagen der Median der Fitfunktion $F(\lambda)$. Oder anders ausgedrückt: diese Wellenlänge ist so berechnet, dass alle Wellenlängen kleiner als $\lambda_{m}$ genau soviel Wärme konvektieren wie Wellenlängen größer als $\lambda_{m}$.

Mit diesem Verfahren wurden sämtliche Simulationen ausgewertet. Das Ergebnis ist in Abbildung 5.29 wieder über $P e_{\text {rot }}$ aufgetragen. Dabei wurde auf der Ordinate $\lambda_{m} E k^{-1 / 3}$ verwendet, um die unterschiedlichen Onset-Wellenlängen für verschiedene Ekman-Zahlen zu kompensieren (siehe Kapitel 2.3). Es sind ebenso wieder die drei Bereiche eingezeichnet, die schon mithilfe von Abbildung 5.10 identifiziert wurden.

Die kritische Wellenlänge bei Konvektionströmungen unter dem Einfluss der Rotation hängt 
nach Gleichung (2.10) von der Ekman-Zahl ab wie:

$$
\lambda_{c}=3.83 E k^{1 / 3}
$$

In der kompensierten Auftragung sollte also im rotationsdominierten Regime dieser Vorfaktor gerade 3.83 sein, unabhängig von der Rotationsrate. Wie man sieht ist das gut erfüllt für die kleinen Ekman-Zahlen. Die größte Ekman-Zahl von $E k=2 \cdot 10^{-1}$ erfüllt das nicht. Allerdings ist für sie auch das Potenzgesetz nach Gleichung (2.10) nicht gültig, wie man gut in Abbildung 2.3 rechts sieht.

Im Transitionsbereich bleibt die Wellenlänge über einen weiten Bereich hin konstant. Mit größer werdendem $P e_{r o t}$ wird die Wellenlänge dann kleiner. Dies stimmt überein mit den Experimenten in [39]. Die weitere Entwicklung sollte im rotationsfreien Bereich natürlich unabhängig von der Ekman-Zahl sein. In Abbildung 5.29 wird die Abhängigkeit für große $P e_{\text {rot }}$ gut durch ein Potenzgesetz mit Steigung $-\frac{1}{2}$ genähert, wie man am eingezeichneten Fit erkennt.

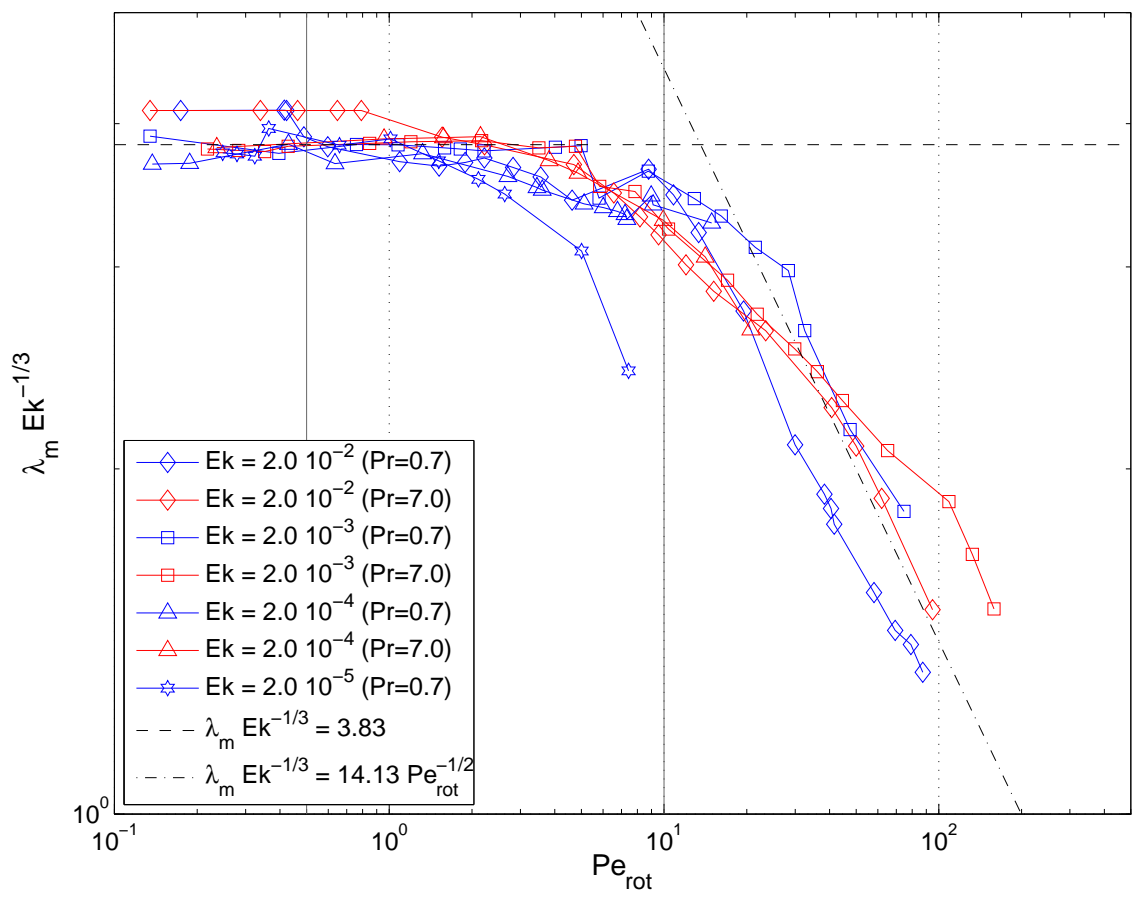

Abb. 5.29: Auftragung der repräsentativen Wellenlänge $\lambda_{m}$ bei $z=0.5$ in der Mitte der Fluidschicht. Eingezeichnet sind Fits für den Bereich des Onsets und den nicht-rotierenden Bereich.

Damit ergibt sich für $\lambda_{m}$ :

$$
\lambda_{m}=(\operatorname{RePr})^{-1 / 2} E k^{1 / 12}
$$


Die Wellenlänge verhält sich also proportional zu $P e^{-1 / 2}$. Die kleine Abhängigkeit von der Ekman-Zahl spielt dabei keine Rolle mehr.

Wie weiter oben erwähnt, wurde das Aspektverhältnis immer so gewählt, dass sowohl in $x$ als auch in $y$-Richtung vier Rollenpaare in das Simulationsgebiet passen. Das Aspektverhältnis wurde also proportional zu $\lambda_{c}$ gewählt. Diese Wahl ist natürlich nur so lange gerechtfertigt, bis die Strömung anfängt größere Strukturen auszubilden.

In Abschnitt 5.4.1 wurde gezeigt, dass sich die Strömung ab $R e_{\text {rot }} \approx 50$ so verhält, als wäre keine Corioliskraft mehr vorhanden. Dies hat zur Folge, dass auch die Struktur der Strömung, mithin die dominante Wellenlänge nicht mehr von $\lambda_{c}$ abhängen sollte. Im Gegenteil, man würde Rollen erwarten, die so groß/breit sind wie im nichtrotierenden Fall. Um das zu verifizieren, wurde eine Simulation, die den nichtrotierenden Zustand schon erreicht haben sollte, bei einem größeren Aspektverhältnis nochmal gerechnet. Es handelt sich um die Simulation mit $E k=2 \cdot 10^{-3}$, $R a=5 \cdot 10^{7}, \operatorname{Pr}=0.7, R e_{r o t} \approx 106$, die zuerst bei $\Gamma=3.0$ durchgeführt wurde. Das resultierende Spektrum ist in Abbildung 5.30 (links) zu sehen.

In Abbildung 5.30 (rechts) ist das Spektrum mit den gleichen Simulationsparametern, aber mit einem doppelt so großem Aspektverhältnis von $\Gamma=6.0$, zu sehen. Die beiden gestrichelten Linien markieren gerade die beiden Aspektverhältnisse. Der Unterschied ist leicht zu erkennen: Die Strömung möchte bei $\Gamma=3.0$ größere Rollen ausbilden, als es möglich ist. Der Hauptteil des konvektiven Wärmetransports muss deshalb durch kleine Strukturen geleistet werden. Die Nusseltzahl ist um knapp 3 Prozent höher als im Fall mit großem Aspektverhältnis.
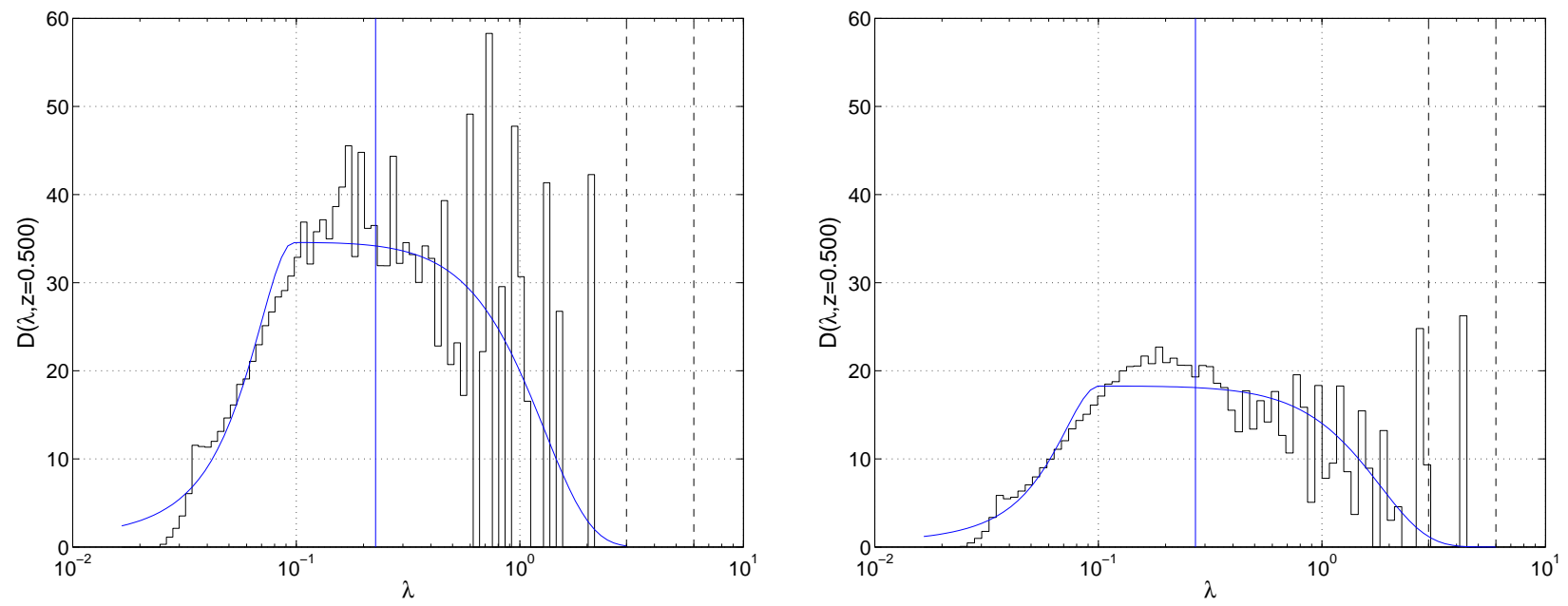

Abb. 5.30: Vergleichende Spektren einer Simulation mit den Paramtern $E k=2 \cdot 10^{-3}, R a=5 \cdot 10^{7}$, $\operatorname{Pr}=0.7, R e_{\text {rot }} \approx 106$ bei $\Gamma=3.0$ (links) und $\Gamma=6.0$ (rechts).

Die Strömung bei $\Gamma=6.0$ zeigt zwei große Wellenlängen rechts im Spektrum, die bei kleinem 
Aspektverhältnis nicht zu sehen waren. Dadurch tragen die kleinskaligen Strukturen weniger zum Wärmetransport bei. Auch die gefittete Wellenlänge $\lambda_{m}$ (durch die senkrechte blaue Linie markiert) verschiebt sich leicht nach rechts.

Es ist also eindeutig zu erkennen, dass die Simulationen, welche im nichtrotierenden Bereich $R e_{\text {rot }} \geq 50$ liegen, bei größerem Aspektverhältnis gerechnet werden müssten, um den geforderten größeren Strukturen Rechnung zu tragen.

Auf den folgenden Seiten werden noch für ausgewählte Rechnungen die spektralen Dichten $D(\lambda, z=0.5)$ und Schnitte durch das Feld des konvektiven Wärmetransports $u_{z} T$ bei $z=$ 0.5 gegenübergestellt. Hierbei ist für jedes einzelne Bild zu einer Kombination aus Rayleigh-, Ekman- und Prandtl-Zahl die Farbskala individuell angepasst. Ein positiver Wärmetransport (in positive $z$-Richtung) ist rot dargestellt, ein negativer Wärmetransport blau.

Dabei können zwei Feststellungen getroffen werden. Erstens sind die Strukturen der Strömungen unterschiedlich für beide Prandtl-Zahlen: Bei $\operatorname{Pr}=0.7$ (entsprechend Luft) sind auch bei großen Rayleigh-Zahlen noch Konvektionsrollen zu beobachten. Dies erkennt man sowohl in den Bildern des konvektiven Wärmetransports, als auch in den Spektren, wo im Vergleich zur größeren Prandtl-Zahl $\operatorname{Pr}=7.0$ (entsprechend Wasser) große Wellenlängen stärker ausgeprägt sind. Damit einhergehend sind die Spektren der kleinskaligen und hauptsächlich die Wärme transportierenden Strukturen im Fall $\mathrm{Pr}=7.0$ höher (es ist zu beachten, dass das strenggenommen nur gilt, wenn die Nusselt-Zahl in beiden Fällen identisch ist. Für kleine Ekman-Zahlen, siehe Abbildung 5.37, ist das nicht mehr der Fall, da dort zu einer Rayleigh-Zahl die Nusselt-Zahlen stark abweichen können. Siehe dazu auch Abbildung 5.9).

Zweitens werden die Strukturen mit größer werdender Rayleigh-Zahl immer kleiner, wie schon in Abbildung 5.29 gezeigt. Dies ist sowohl in den Schnitten des konvektiven Wärmetransports an der Verringerung des Durchmessers der farbigen Strukturen zu erkennen, als auch an einer Verschiebung der Spektren zu kleineren Wellenlängen hin. Die gefittete Wellenlänge $\lambda_{m}$ ist als gestrichelte Linie mit eingetragen. 

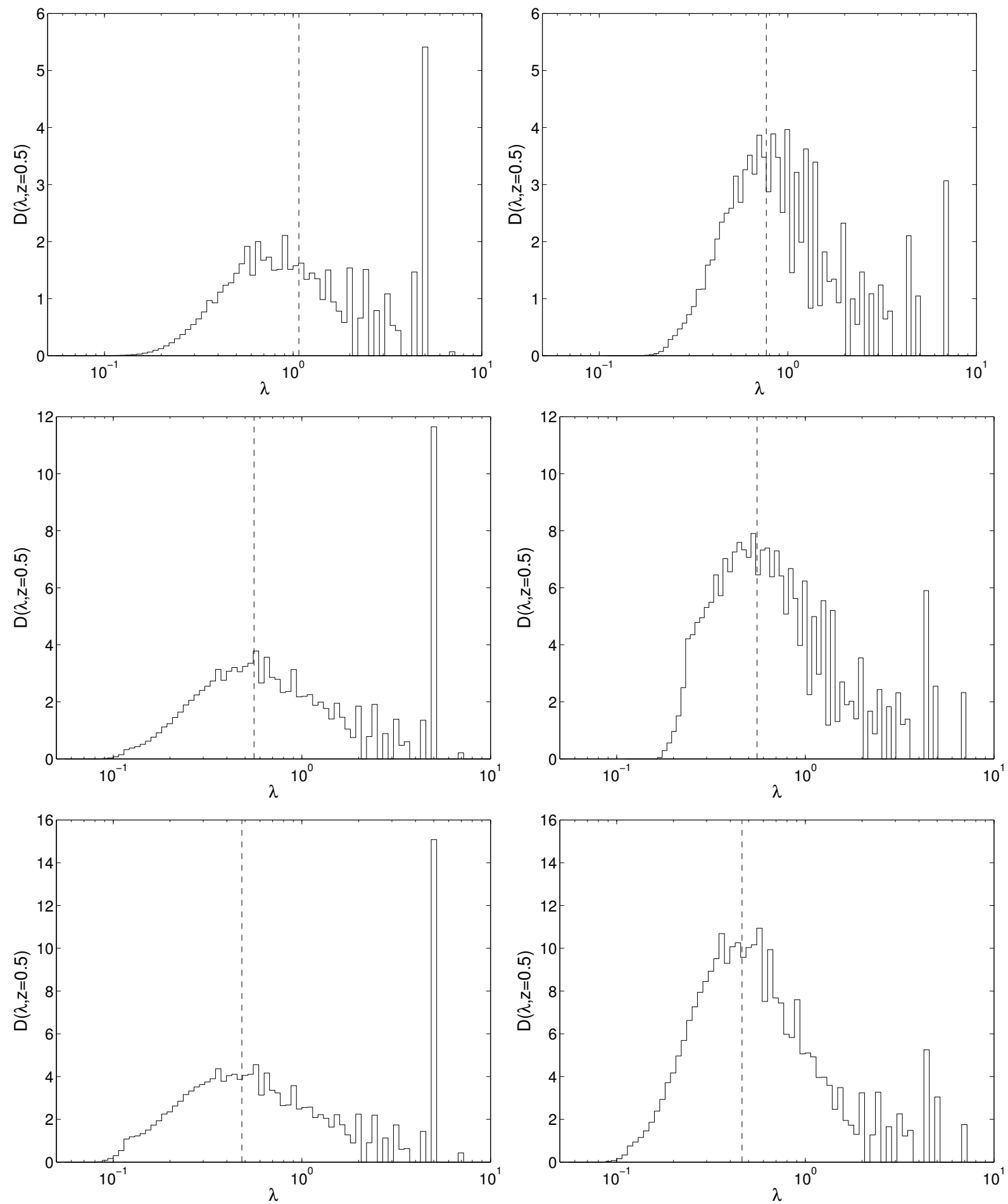

Abb. 5.31: Spektrale Dichte des Wärmetransports für $E k=\infty$ bei $z=0.5$ mit $R a=1 \cdot 10^{5}$ (obere Zeile), $R a=7 \cdot 10^{5}$ (mittlere Zeile) und $R a=1 \cdot 10^{6}$ (untere Zeile). Die Prandtl-Zahlen sind $\operatorname{Pr}=0.7$ links und $\operatorname{Pr}=7.0$ rechts. 

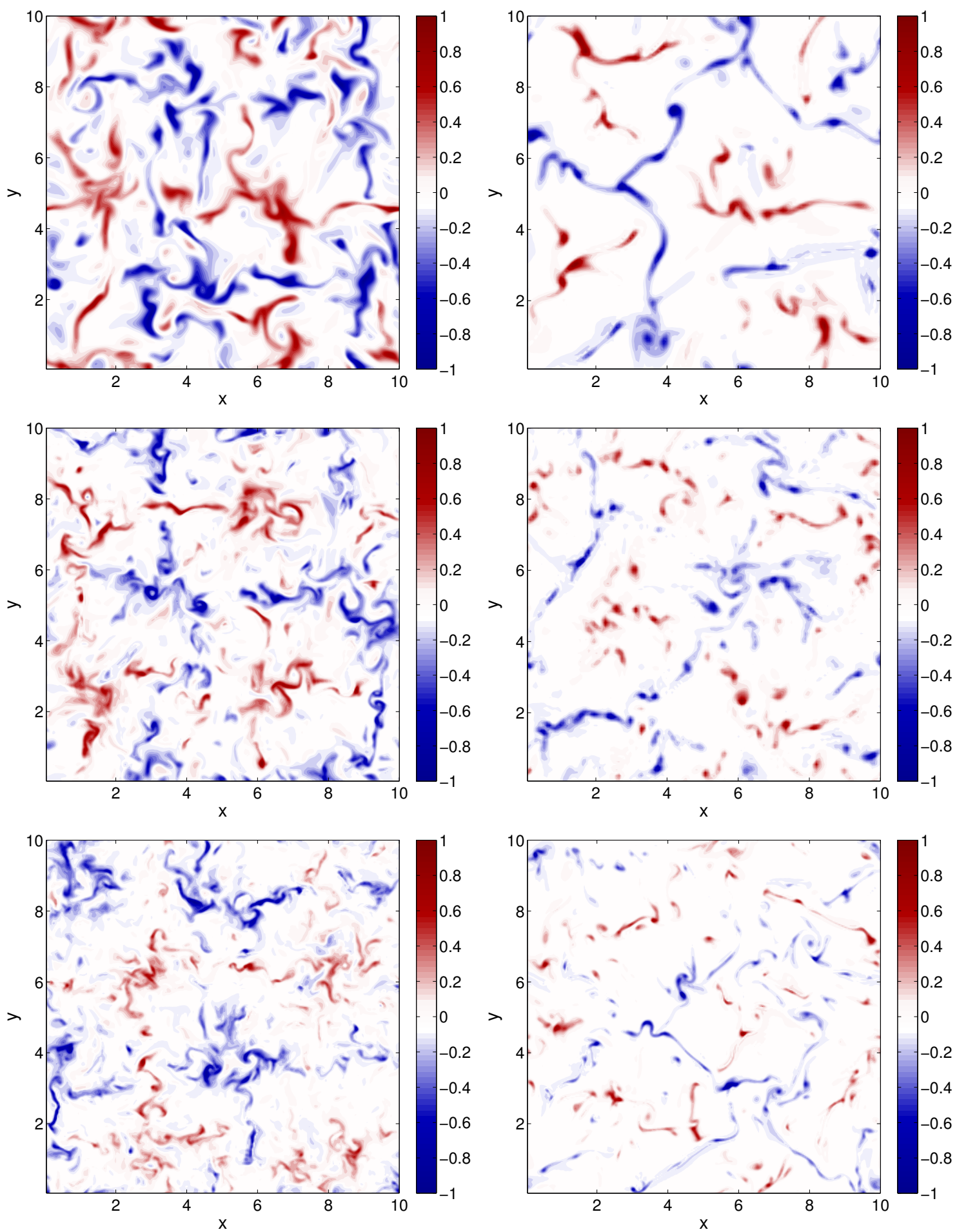

Abb. 5.32: Schnitte des konvektiven Wärmetransports für $E k=\infty$ bei $z=0.5$ mit $R a=1 \cdot 10^{5}$ (obere Zeile), $R a=7 \cdot 10^{5}$ (mittlere Zeile) und $R a=1 \cdot 10^{6}$ (untere Zeile). Die Prandtl-Zahlen sind $\operatorname{Pr}=0.7$ links und $\operatorname{Pr}=7.0$ rechts. 

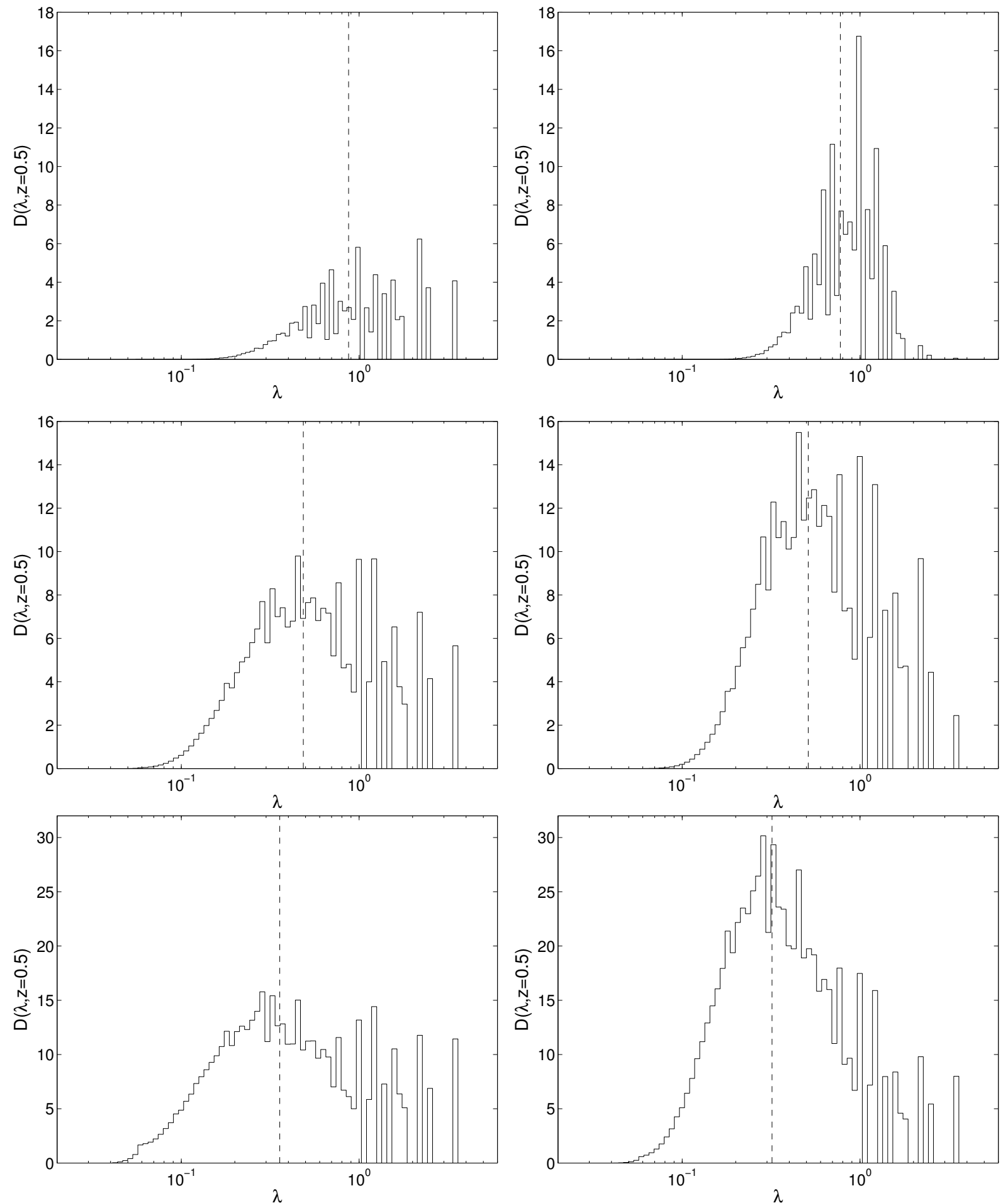

Abb. 5.33: Spektrale Dichte des Wärmetransports für $E k=2 \cdot 10^{-2}$ bei $z=0.5$ mit $R a=1 \cdot 10^{5}$ (obere Zeile), $R a=1 \cdot 10^{6}$ (mittlere Zeile) und $R a=5 \cdot 10^{6}$ (untere Zeile). Die Prandtl-Zahlen sind $\operatorname{Pr}=0.7$ links und $\operatorname{Pr}=7.0$ rechts. 

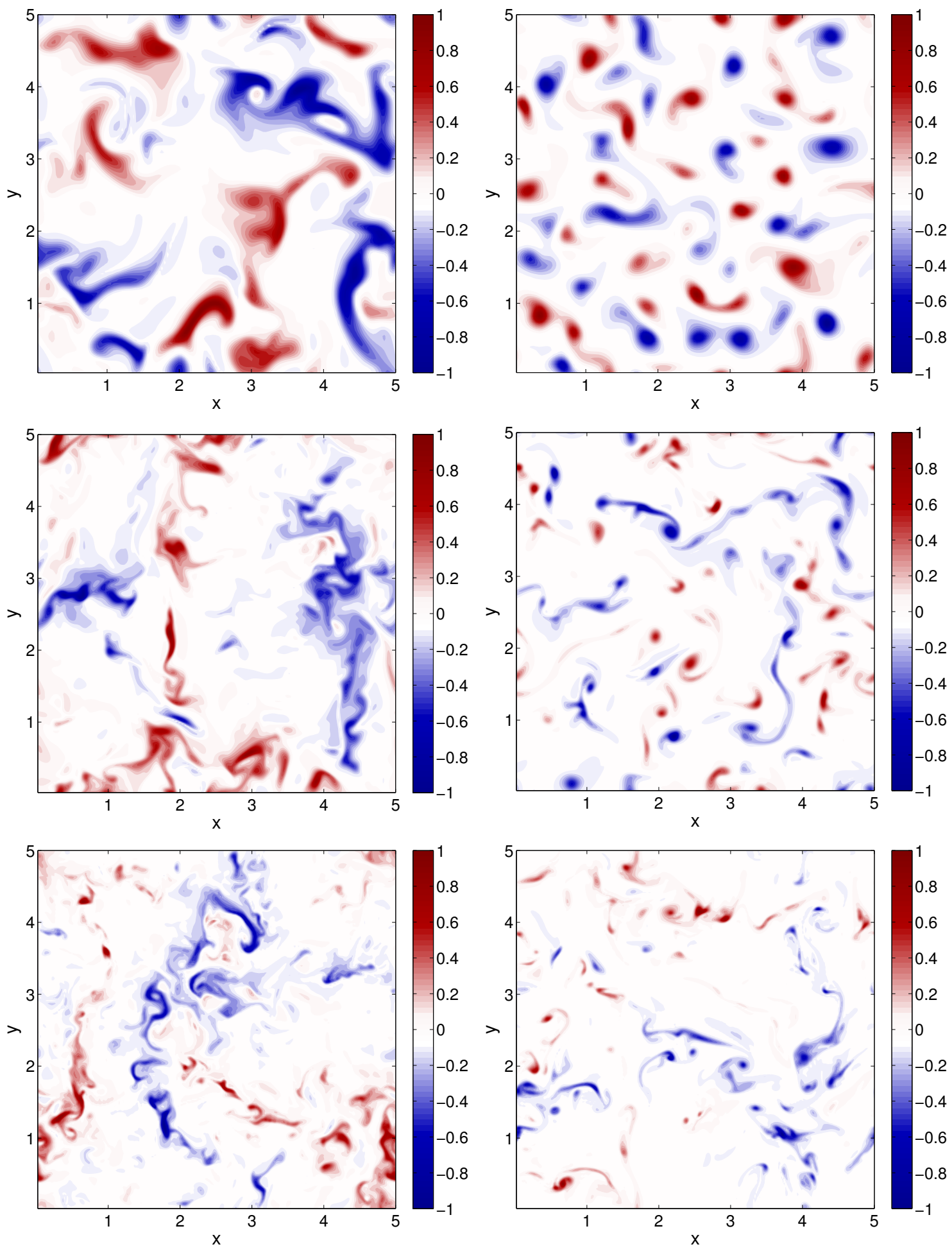

Abb. 5.34: Schnitte des konvektiven Wärmetransports für $E k=2 \cdot 10^{-2}$ bei $z=0.5$ mit $R a=1 \cdot 10^{5}$ (obere Zeile), $R a=1 \cdot 10^{6}$ (mittlere Zeile) und $R a=5 \cdot 10^{6}$ (untere Zeile). Die PrandtlZahlen sind $\operatorname{Pr}=0.7$ links und $\operatorname{Pr}=7.0$ rechts. 

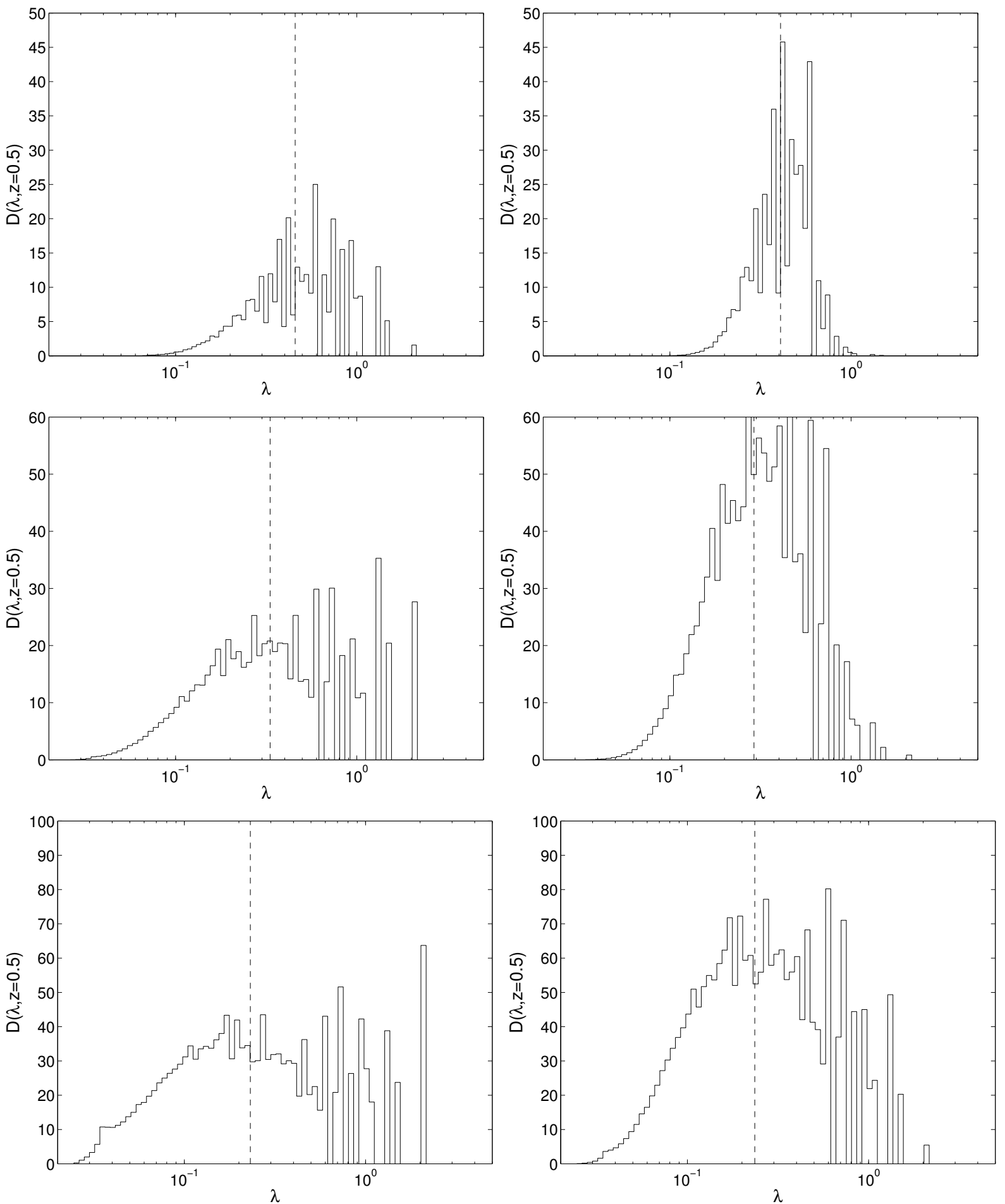

Abb. 5.35: Spektrale Dichte des Wärmetransports für $E k=2 \cdot 10^{-3}$ bei $z=0.5$ mit $R a=1 \cdot 10^{6}$ (obere Zeile), $R a=1 \cdot 10^{7}$ (mittlere Zeile) und $R a=5 \cdot 10^{7}$ (untere Zeile). Die Prandtl-Zahlen sind $\operatorname{Pr}=0.7$ links und $\operatorname{Pr}=7.0$ rechts. 

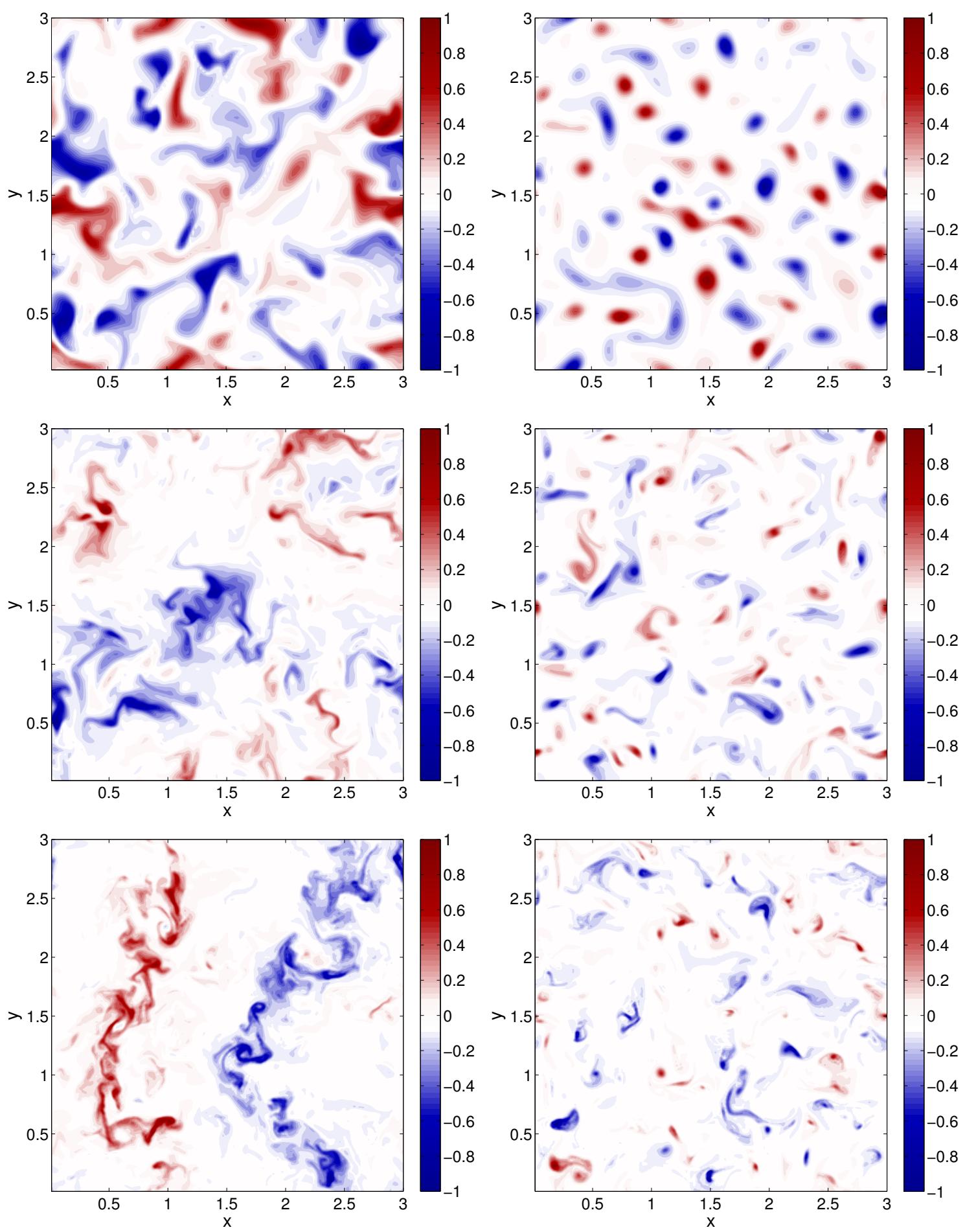

Abb. 5.36: Schnitte des konvektiven Wärmetransports für $E k=2 \cdot 10^{-3}$ bei $z=0.5$ mit $R a=1 \cdot 10^{6}$ (obere Zeile), $R a=1 \cdot 10^{7}$ (mittlere Zeile) und $R a=5 \cdot 10^{7}$ (untere Zeile). Die PrandtlZahlen sind $\operatorname{Pr}=0.7$ links und $P r=7.0$ rechts. 
Kapitel 5. Ergebnisse
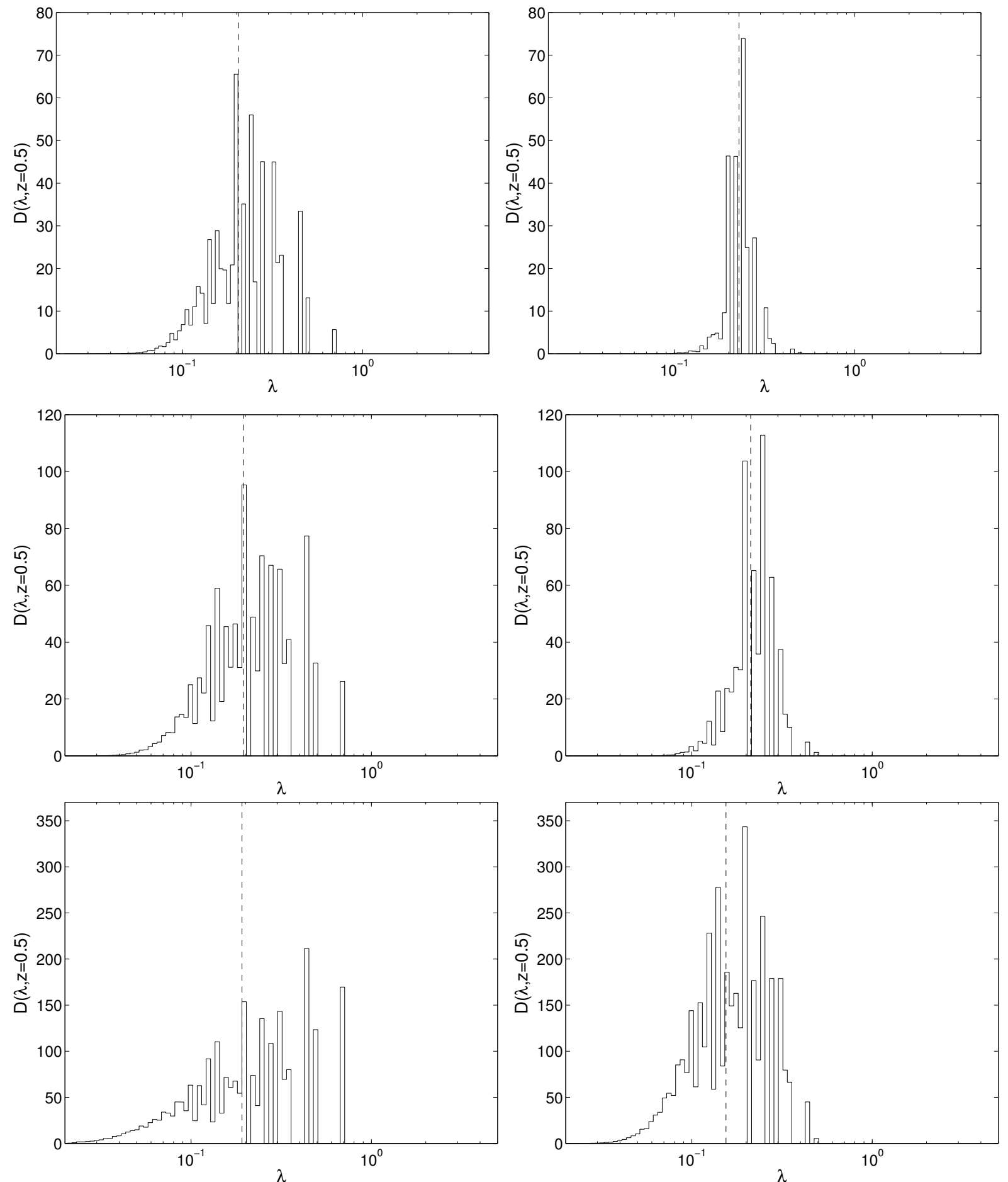

Abb. 5.37: Spektrale Dichte des Wärmetransports für $E k=2 \cdot 10^{-4}$ bei $z=0.5$ mit $R a=5 \cdot 10^{6}$ (obere Zeile), $R a=1 \cdot 10^{7}$ (mittlere Zeile) und $R a=5 \cdot 10^{7}$ (untere Zeile). Die Prandtl-Zahlen sind $\operatorname{Pr}=0.7$ links und $\operatorname{Pr}=7.0$ rechts. 

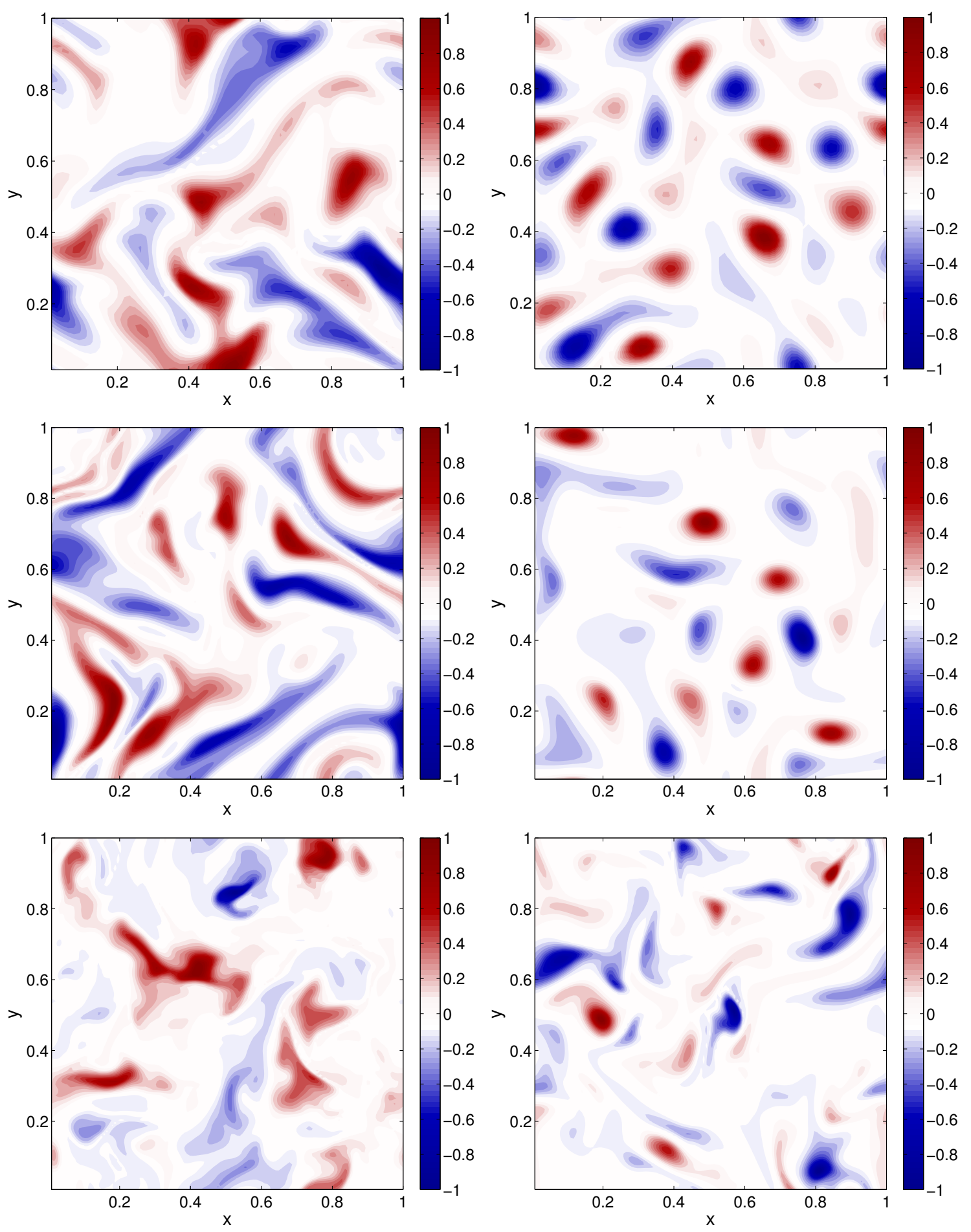

Abb. 5.38: Schnitte des konvektiven Wärmetransports für $E k=2 \cdot 10^{-4}$ bei $z=0.5$ mit $R a=1 \cdot 10^{6}$ (obere Zeile), $R a=5 \cdot 10^{7}$ (mittlere Zeile) und $R a=5 \cdot 10^{7}$ (untere Zeile). Die PrandtlZahlen sind $\operatorname{Pr}=0.7$ links und $\operatorname{Pr}=7.0$ rechts. 


\subsection{Diskussion der Ergebnisse}

In diesem fünften Kapitel wurden die Ergebnisse der Arbeit vorgestellt. Ein wichtiges Resultat ist, dass der konvektive Wärmetransport $N u-1$, über der Péclet-Zahl $P e$ bzw. P $e_{\text {rot }}$ aufgetragen, unabhängig von den Randbedingungen ist. Es ist also eine universelle Auftragung. Sie liefert für Simulationen ohne und mit Rotation in beiden Bereichen, sowohl am Onset als auch für hohe (Rotations-) Péclet-Zahlen, das gleiche asymptotische Verhalten, siehe Abbildung 5.10. Leider konnte letztlich nicht geklärt werden, welches Potenzgesetz im zweiten Bereich das richtige ist. Dazu liegen dort zu wenig Daten vor. Die wenigen Daten, die es gibt, müssen auch mit Vorsicht betrachtet werden. Das Aspektverhältnis wurde, in Abhängigkeit von der Ekman-Zahl, immer so gewählt, dass 4 Rollenpaare in die Zelle passten. Wie bei der Analyse der Spektren gezeigt wurde, reicht dieses Aspektverhältnis aber nicht mehr aus, wenn die Strömung in den nichtrotierenden Bereich kommt.

Für rotationsbehaftete Strömungen tritt noch ein dritter Bereich zutage. In diesem Transitionsbereich zeigt sich für schubspannungsfreie Ränder eine Aufspaltung der Werte, siehe Abbildung 5.10. Dies ist bei den Werten aus Simulationen mit no-slip Rändern nicht zu erkennen. Ob das an der eingeschränkten Spanne von Ekman-Zahlen liegt, oder an den Randbedingungen selbst, müsste durch weitere Untersuchungen geklärt werden. Allerdings sieht man, dass im Transitionsbereich Diffusivitäten keine Rolle spielen. Durch die geeignete Kombination der Kennzahlen entstehen andere, diffusionsfreie Kennzahlen. In einer Auftragung der diffusionsfreien Nusselt-Zahl über der diffusionsfreien Fluss-Rayleigh-Zahl findet man ein Potenzgesetz mit einem Exponenten nahe bei $\frac{5}{9}$ bzw. $\frac{6}{11}$. Es ist (bis auf den Vorfaktor) identisch für beide Randbedingungen. Darüber hinaus stößt man auf den gleichen Exponenten auch in anderen Untersuchungen (siehe [5]), sowohl numerischen als auch experimentellen. Diese fanden sowohl in ebener Geometrie mit vertikal orientierter Schwerebeschleunigung (wie in der vorliegenden Arbeit) als auch z.B. in Kugelgeometrien mit radial veränderlicher Schwerebeschleunigung statt. Es ist deshalb verblüffend, dass der Exponent für solch verschiedene Rahmenbedingungen allgemeingültig ist.

Als Beispiel sollen die diffusionsfreien Kennzahlen für den Erdkern abgeschätzt werden. Als Winkelgeschwindigkeit nimmt man $\Omega=7.29 \cdot 10^{-5} \frac{1}{s}$ an. Die weiteren Werte sind nach [28] $g=$ $10 \frac{\mathrm{m}}{\mathrm{s}^{2}}, \alpha=2 \cdot 10^{-5} \frac{1}{\mathrm{~K}}, Q=4 \cdot 10^{12} \mathrm{~W}, \Delta T=1000 \mathrm{~K}, \varrho=1 \cdot 10^{4} \frac{\mathrm{kg}}{\mathrm{m}^{3}}, c_{p}=700 \frac{\mathrm{J}}{\mathrm{kgK}}$ und $d=2286 \cdot 10^{3} \mathrm{~m}$. Damit ergibt sich $R a_{f *} \approx 5 \cdot 10^{-13}$ und $N u_{*} \approx 2 \cdot 10^{-14}$. Solch kleine Kennzahlen wurden in den vorliegenden Simulationen nicht erreicht. Andere Ergebnisse kommen schon nahe an $R a_{f *} \approx$ $1 \cdot 10^{-10}$, wie in Abbildung 5.19 zu sehen. Dies allerdings nur mit Parameterkombinationen, die keinen Bezug zum realen Erdkern haben, also z.B. Ekman-Zahlen von $1 \cdot 10^{-5}$. 
Die Auftragung des konvektiven Wärmetransports ermöglicht es also zu entscheiden, ab wann selbiger nicht mehr durch die Rotation beeinflusst wird. Das ist dann der Fall, wenn das asymptotische Verhalten des nichtrotierenden Wärmetransports erreicht wird. In dieser Arbeit wurde diese Grenze mit $P e_{\text {rot }} \approx 10$ abgeschätzt. Es ist also möglich, diese Grenze zu bestimmen ohne Kenntnis der Rayleigh-Zahl. Statt des Temperaturgradienten bzw. des Wärmeflusses muss „nur" die Geschwindigkeit bekannt sein.

Auch für $P e_{\text {rot }}$ lässt sich daraus eine Abschätzung vornehmen. Aus den Messungen der magnetischen Säkularvariation ergibt sich als typische Geschwindigkeit $u=5 \cdot 10^{-4} \frac{\mathrm{m}}{\mathrm{s}}$. Mit den Materialkonstanten (aus [36]) von $\kappa=3 \cdot 10^{-6} \frac{\mathrm{m}^{2}}{\mathrm{~s}}$ und $\nu=5 \cdot 10^{-7} \frac{\mathrm{m}^{2}}{\mathrm{~s}}$ ergibt sich $P e_{\text {rot }} \approx 13$. Der Erdkern liegt also knapp außerhalb des Transitionsbereichs. Nimmt man für den Erdkern hingegen kompositionelle Konvektion an mit einer thermischen Diffusivität von $\kappa=7 \cdot 10^{-9} \frac{\mathrm{m}^{2}}{\mathrm{~s}}$, so ergibt sich $P e_{\text {rot }} \approx 6 \cdot 10^{3}$.

Daneben wurde eine Möglichkeit hergeleitet, eine solche Grenze auch für die Struktur (bzw. die Geschwindigkeit) der Strömung anzugeben. Da die Strömung durch den Einfluss der Rotation zu helikalen Strömungsmustern gezwungen wird, bot sich die Untersuchung der normierten Helizität an. Simulationen ohne Rotation zeigten einen sehr kleinen rms-Wert der ebenengemittelten normierten Helizität. Dieser wurde als Schranke definiert. Für Simulationen mit Rotation liefert ein Vergleich mit dieser Schranke ein Kriterium, ab dem die Strömung als rotationsfrei gelten kann. Für die schubspannungsfreien Randbedingungen wurde die Rotations-ReynoldsZahl als Maß dafür gefunden, für no-slip Ränder wurde diese Auswertung aufgrund fehlender Daten nicht durchgeführt. Ab der Grenze $R e_{\text {rot }} \approx 50$ kann die Strömung als rotationsfrei genähert werden. Sie ist unabhängig von den thermischen Eigenschaften des Fluids. Die Auswertung der kinetischen Energiedissipation unterstützt die Gültigkeit der gefundenen Grenze. Für das Beispiel des Erdkerns oben ergibt sich $R e_{\text {rot }} \approx 82$.

Die beiden Fragestellungen nach einer Grenze zum rotationsfreien Fall für den Wärmetransport bzw. die Strömungsstruktur selbst finden die Antworten also in der Rotations-Péclet- bzw. Rotations-Reynolds-Zahl. Beide unterscheiden sich nur in der Prandtl-Zahl, die wiederum nur vom Fluid selbst abhängt. Diese Kriterien, die beide von $R e E k^{1 / 2}$ abhängen, stehen im Widerspruch zur Rossby-Zahl $R o=R e E k$. Sie ist das Verhältnis aus Trägheits- und Corioliskraft. Die klassische Annahme, dass die Grenze zwischen rotationsdominiertem und rotationsfreiem Bereich bei $R o=R e E k=1$ liegt, stimmt mit den in dieser Arbeit gewonnenen Erkenntnissen nicht überein.

Auf der anderen Seite sind aber auch $R e_{\text {rot }}$ bzw. $P e_{\text {rot }}$ für Simulationen mit schubspannungsfreien Rändern keine offensichtlichen Größen. Für no-slip Ränder haben die Herleitungen der 
beiden Kennzahlen ihre Begründung in den Ekman-Schichten, die proportional zu $E k^{1 / 2}$ sind. Dies untermauern auch die Resultate der entsprechenden Rechnungen. In den schubspannungsfreien Simulationen konnte eine Länge, die mit $E k^{1 / 2}$ skaliert, allerdings nicht gefunden werden. Die thermischen Grenzschichten zeigen ein Verhalten, das unabhängig von den Rändern ist. Außerhalb dieser Grenzschichten stellt sich für Simulationen ohne Rotation ein konstantes Temperaturprofil ein, wenn die Strömung nur überkritisch genug ist. Kommt die Rotation hinzu, so bewirkt sie (zumindest für nicht zu große Ekman-Zahlen, ab etwa $E k \leq 2 \cdot 10^{-3}$ ) eine Verzögerung, das Temperaturprofil wird erst später konstant. Auch ist der Weg hin zu diesem konstanten Profil abhängig von der Prandtl-Zahl.

Eine Abhängigkeit von der Prandtl-Zahl zeigen auch die Spektren des konvektiven Wärmetransports. Bei der kleineren Prandtl-Zahl von $\operatorname{Pr}=0.7$ sind große Strukturen ausgeprägter als bei $\operatorname{Pr}=7.0$. Das sieht man am besten für hohe Rayleigh-Zahlen, wenn die Strömung schon (fast) das nichtrotierende Regime erreicht hat. Nahe am Onset dominieren natürlich Strukturen in der Größenordnung der kritischen Wellenlänge. Dies konnte nachgewiesen werden, indem an die Spektren ein Fit angepasst wurde, der diesen kleinen Strukturen objektiv eine einzige, repräsentative Wellenlänge $\lambda_{m}$ zuordnet. Eine Auftragung von $\lambda_{m}$ über der Rotations-Péclet-Zahl liefert wieder für verschiedene Prandtl- bzw. Ekman-Zahlen eine universelle Auftragung: nahe am Onset ist die Wellenlänge konstant und entspricht der berechneten Onset-Wellenlänge und für den rotationsfreien Bereich wird die Wellenlänge kleiner, wie man es erwartet. 


\section{Kapitel 6}

\section{Zusammenfassung}

In der vorliegenden Arbeit wurden Konvektionsströmungen unter dem Einfluss von Rotation untersucht. Dazu wurde das bestehende Programm mpplconv aus [19] erweitert um den Coriolisterm. Ebenso wurde eine neue Zeitschrittberechnung implementiert, um schubspannungsfreie Randbedingungen verwenden zu können.

Die Simulationen für den Fall ohne Rotation zeigen, sowohl für die Reynolds- als auch für die Nusselt-Zahl, die erwarteten Ergebnisse. In einer Auftragung des konvektiven Wärmetransports $N u-1$ über der Péclet-Zahl $P e$ fallen die Ergebnisse zusammen, unabhängig von der Prandtl-Zahl. Es ist in dieser Auftragung sogar gleichgültig, welche Randbedingungen verwendet wurden.

Im Fall mit Rotation wurde eine dazu äquivalente Darstellung gefunden, bei der alle Ergebnisse für den konvektiven Wärmetransport $N u-1$ für verschiedene Ekman-Zahlen übereinander fallen. Sie ist mit einer Länge proportional zu $E k^{1 / 2}$ gebildet und stellt das Analogon zur bekannten Péclet-Zahl für rotationsfreie Strömungen dar. Auch hierbei zeigte sich das gleiche Verhalten unabhängig von den Randbedingungen. Für Simulationen mit no-slip Rändern, die eine Grenzschichtdicke der Geschwindigkeit proportional zu $E k^{1 / 2}$ aufweisen, ist das nicht erstaunlich. Es wurde aber auch gezeigt, dass es für schubspannungsfreie Ränder keine solche Grenzschicht und mithin keine Länge proportional zu $E k^{1 / 2}$ gibt. Dennoch zeigen auch in diesem Fall die Werte des konvektiven Wärmetransports das gleiche universelle Verhalten.

In der universellen Auftragung tritt, neben den erwarteten Regimes (rotationsdominiert nahe des Onsets für kleine Werte von $P e_{\text {rot }}$ und rotationsfrei für große Werte von $P e_{\text {rot }}$ ) ein Transitionsbereich als drittes Regime zutage. Mithilfe diffusionsfreier Kennzahlen konnte gezeigt werden, dass in diesem Bereich die Diffusion keine Rolle spielt, die Strömung also lediglich durch 
den nichtlinearen Term und den Coriolisterm bestimmt wird. Auch hier ist das Potenzgesetz für beide Randbedingungen das gleiche, lediglich in den Vorfaktoren gibt es Abweichungen.

Die Strömungsstrukturen wurden zum einen qualitativ untersucht. Anhand von Temperaturisoflächen wurde der Einfluss der Prandtl-Zahl auf die Strömung gezeigt. Quantitativ wurden die Strukturen durch die Berechnung der normierten Helizität identifiziert. Rotationsdominierte Strömungen sind durch eine große normierte Helizität gekennzeichnet, während sie in rotationsfreien Strömungen nahezu verschwindet. Daraus wurde ein Kriterium für die RotationsReynolds-Zahl $R e_{r o t}=R e E k^{1 / 2}$ abgeleitet, ab dem eine Strömung als unbeeinflusst von der Corioliskraft gelten kann.

Vergleicht man den Wärmetransport und die Struktur der Strömung, so erkennt man, dass der Übergang zum rotationsfreien Regime nicht identisch ist. Für den Wärmetransport liegt er bei $P e_{\text {rot }} \approx 10$, ist also von der Prandtl-Zahl abhängig. Die Struktur der Strömung erlangt bei $R e_{\text {rot }} \approx 50$ wieder Ähnlichkeit mit dem nichtrotierenden Fall. Sowohl beim Wärmetransport als auch bei der Struktur der Strömung ist der Übergang aber proportional zu $E k^{1 / 2}$.

Zum Anderen wurde die vorherrschende Länge in der Strömung bestimmt mittels des konvektiven Wärmetransports. In der Mittelebene des Simulationsgebiets bei $z=0.5$ wurde die spektrale Dichte $D$ der Größe $u_{z} T$ berechnet. Ein Fit mit einer modifizierten Gaussglocke an diese Auftragung erlaubte die Berechnung einer Wellenlänge $\lambda_{m}$. Diese Größe, wieder aufgetragen über $P e_{\text {rot }}$, zeigt ein ebenso einfaches Bild wie der konvektive Wärmetransport: für den rotationsdominierten und den Transitionsbereich ist $\lambda_{m}$ konstant, für den rotationsfreien Bereich wird sie kleiner, übereinstimmend mit experimentellen Daten. Allerdings wurde anhand einer Vergleichsrechnung auch nachgewiesen, dass im rotationsfreien Regime ein größeres Aspektverhältnis (und damit auch eine höhere Auflösung) erforderlich ist, da dort die Strukturen so groß wie im nichtrotierenden Fall sind. Zum Schluss wurden noch für ausgewählte Kombinationen aus Rayleigh-, Ekman- und Prandtl-Zahlen die spektrale Dichte $D$ und Schnitte des konvektiven Wärmetransports $u_{z} T$ gegenübergestellt. 


\section{Anhang A}

\section{Tabellen der Simulationsergebnisse}

In diesem Anhang sind die Ergebnisse der verschiedenen Simulationen festgehalten. Es gibt zu jeder Kombination von Ekman- und Prandtl-Zahlen eine Tabelle. Jede dieser Tabellen ist nach der Rayleigh-Zahl sortiert.

$\mathrm{Zu}$ jeder Rayleigh-Zahl ist zuerst die verwendete numerische Auflösung $N_{x} \times N_{y} \times N_{z}$ notiert. Danach stehen die beiden wichtigsten Kennzahlen, die Nusselt-Zahl $N u$ und die Reynolds-Zahl $R e$, und die daraus berechneten Größen $P e_{\text {rot }}=\operatorname{Re} \operatorname{Pr} E k^{1 / 2}$ und $\operatorname{Re} E k^{1 / 2}$. Die diffusionsfreien Kennzahlen $R a_{Q}^{*}$ und $N u^{*}$ sind auch protokolliert, ebenso wie die gefittete Wellenlänge $\lambda_{m}$. 


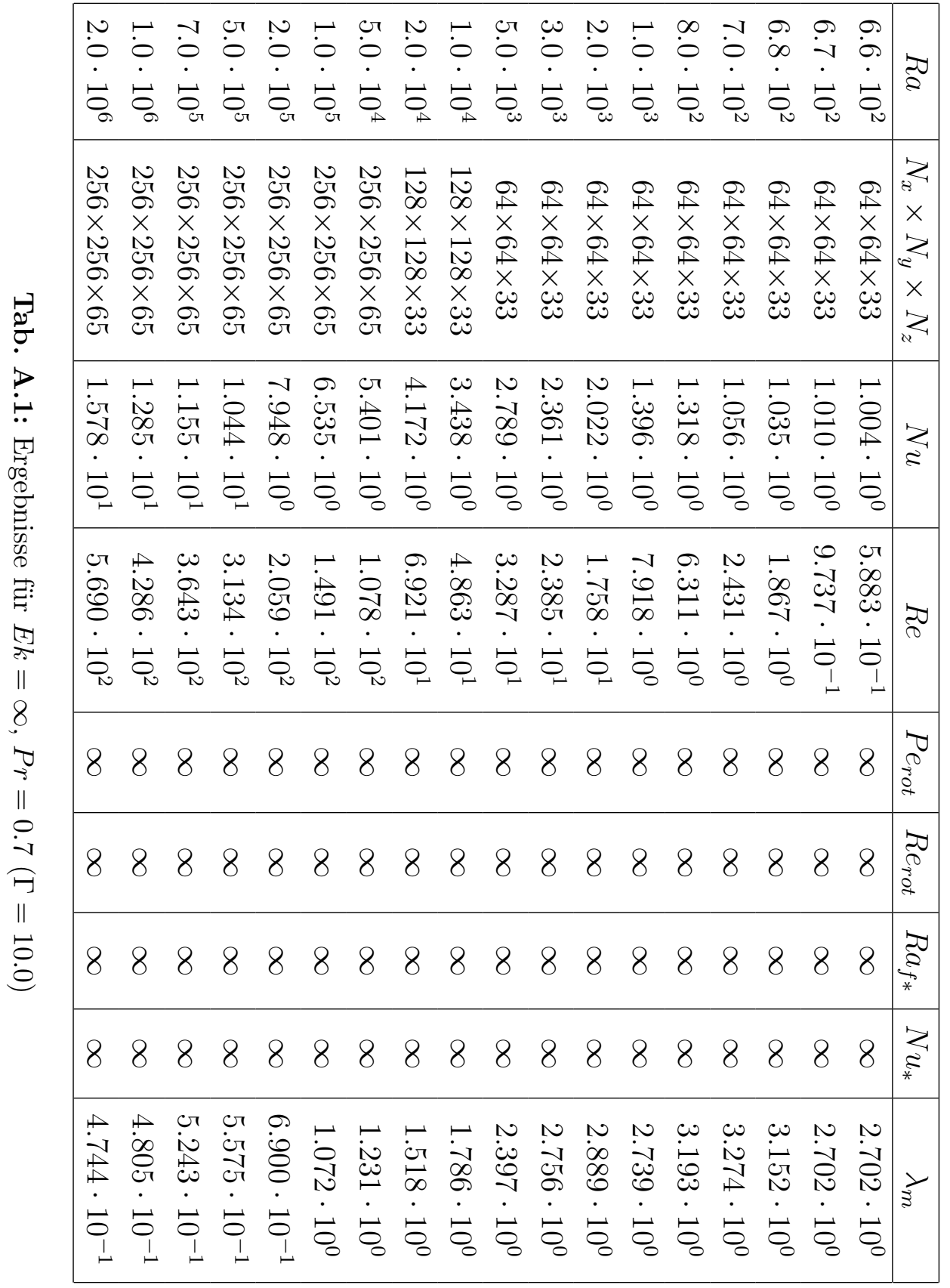




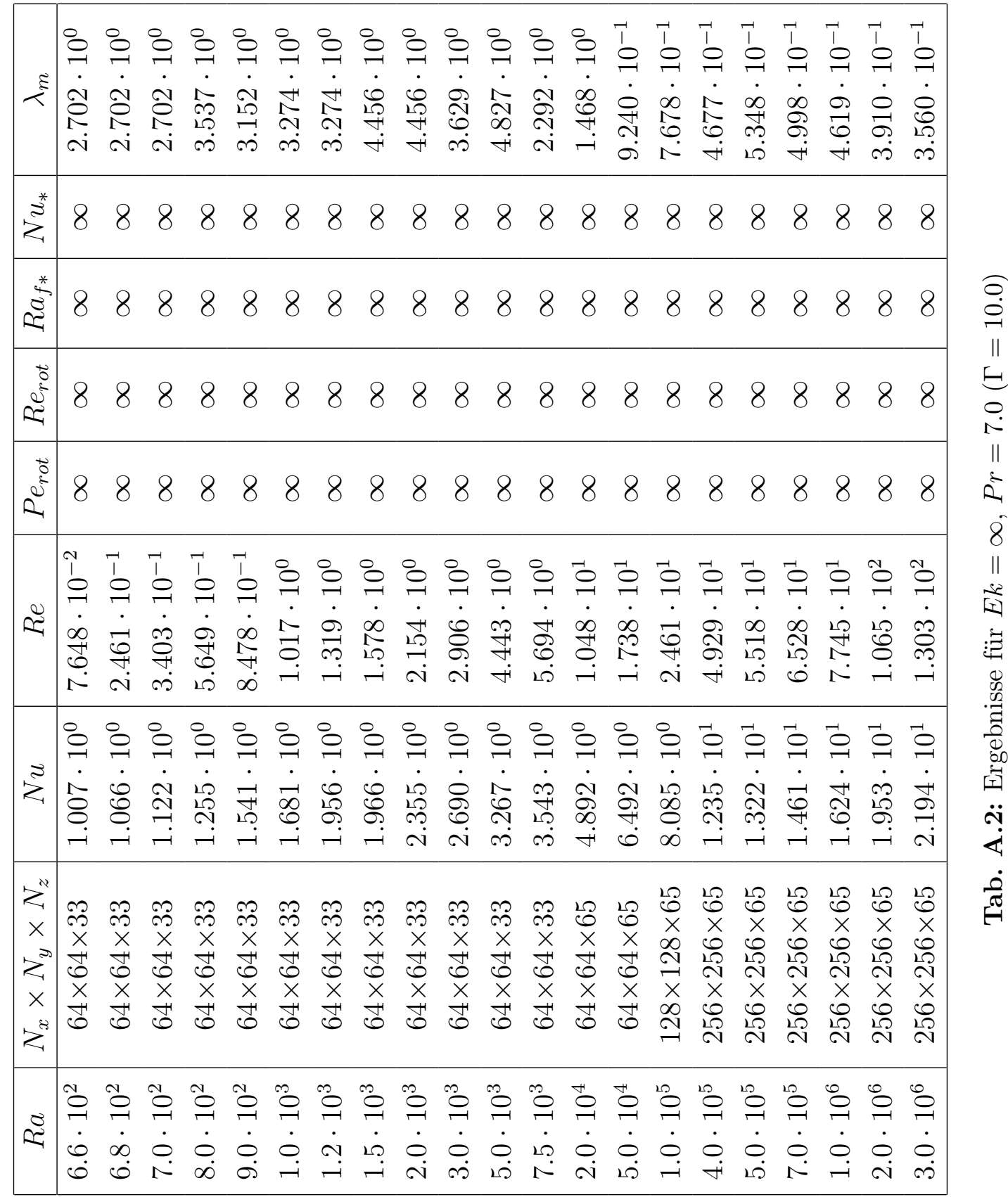




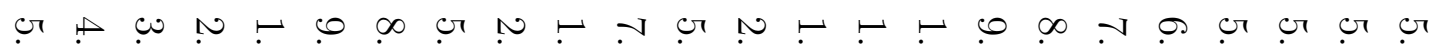

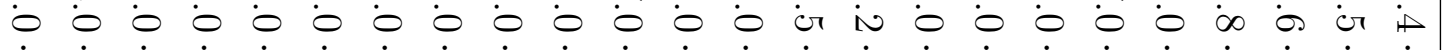

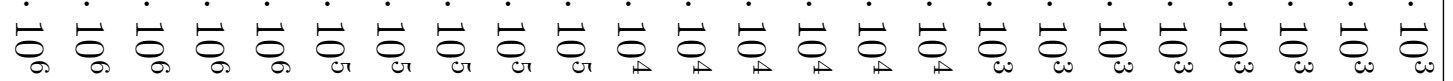

岛

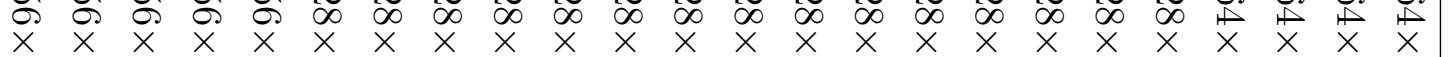

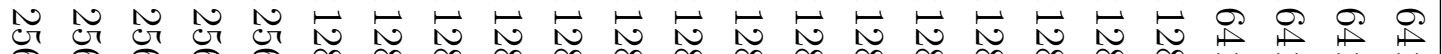

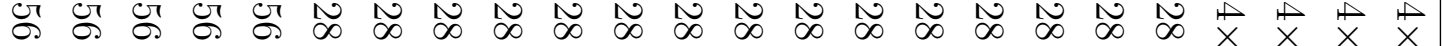

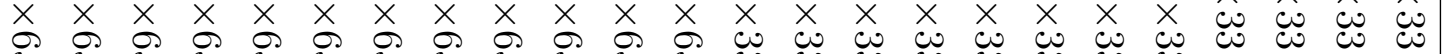

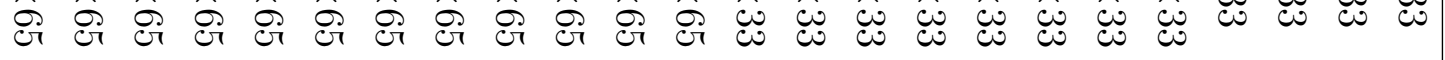

ヘ

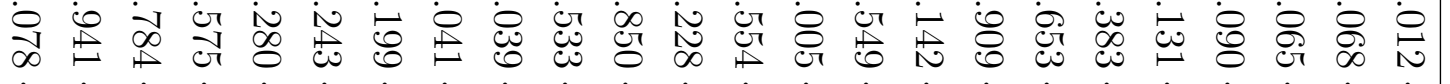
ஜ

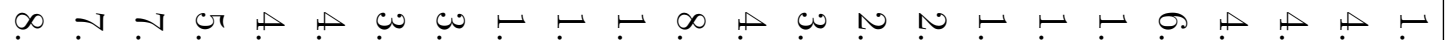

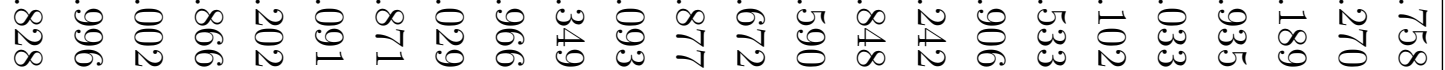
(

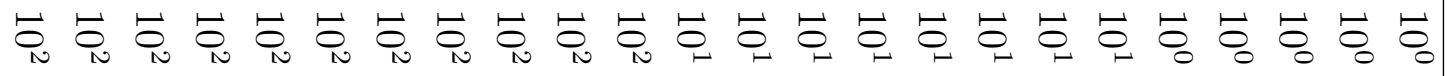

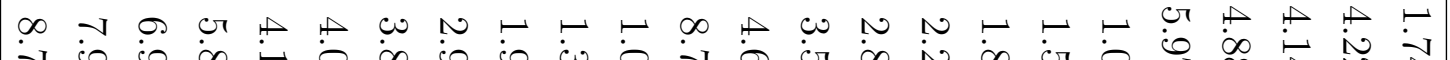

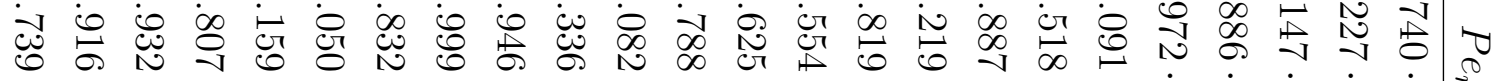

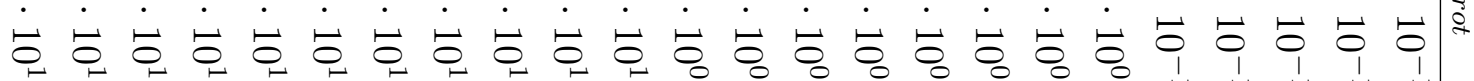

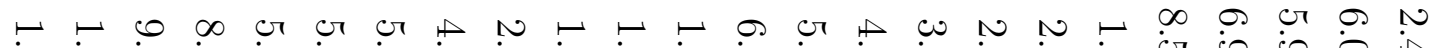

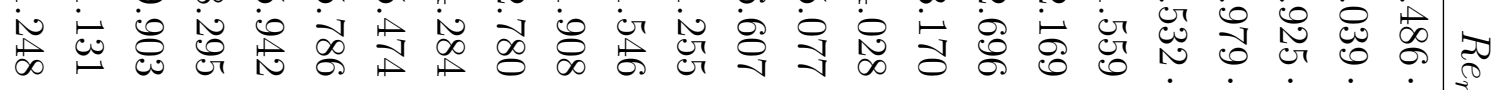

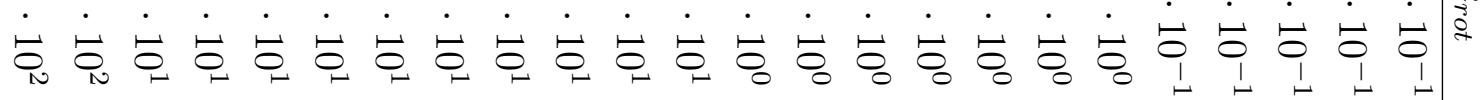

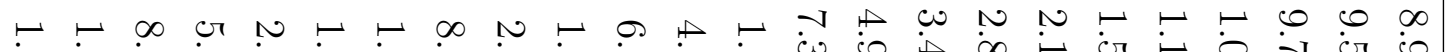

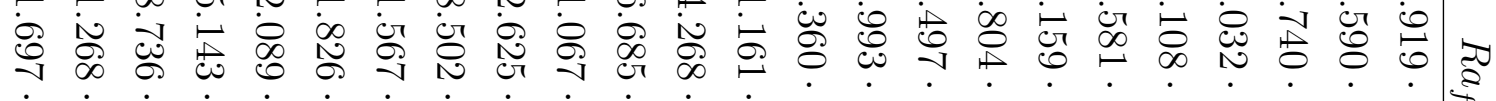

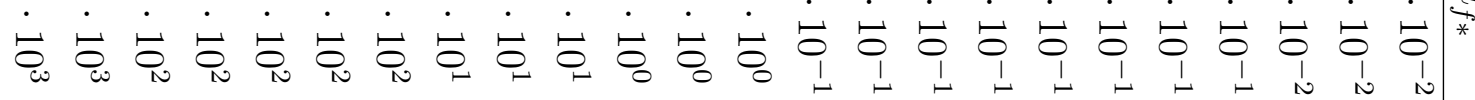

G iv

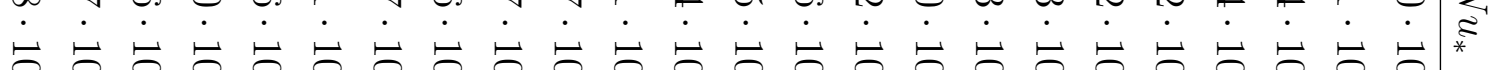

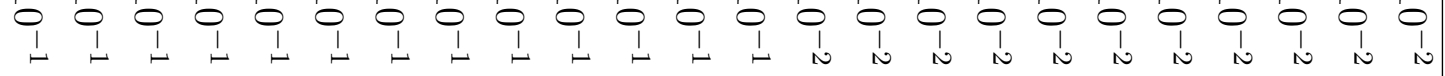

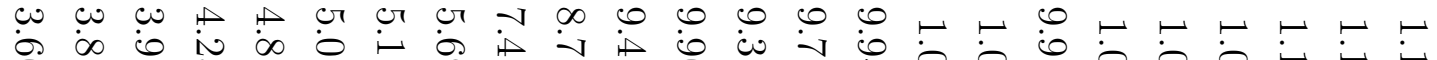

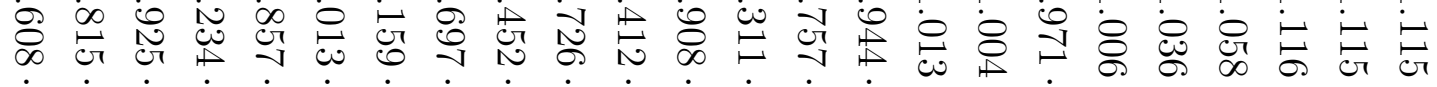

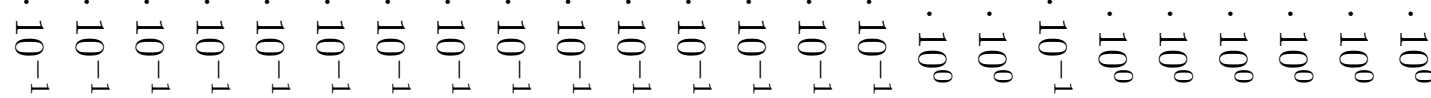




\begin{tabular}{|c|c|c|c|c|c|c|c|c|c|c|c|c|c|c|c|c|c|c|}
\hline है & 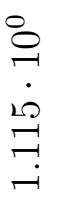 & 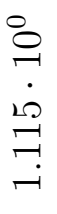 & $\begin{array}{l}\mathscr{\Xi} \\
\ddot{1} \\
\dot{0} \\
ت \\
ت \\
ت\end{array}$ & $\begin{array}{l}\stackrel{8}{0} \\
\because \\
\dot{10} \\
ت \\
ت \\
ت\end{array}$ & 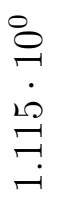 & $\begin{array}{l}\stackrel{0}{0} \\
\cdot \\
\sim \\
\stackrel{0}{0} \\
ت\end{array}$ & 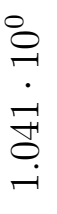 & 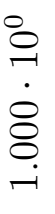 & 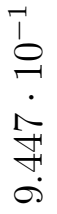 & $\begin{array}{l}7 \\
0 \\
1 \\
\dot{1} \\
\stackrel{8}{0} \\
0 \\
0\end{array}$ & $\begin{array}{l}1 \\
1 \\
0 \\
\dot{1} \\
0 \\
\infty \\
0 \\
\infty \\
\infty\end{array}$ & 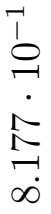 & $\begin{array}{l}\overrightarrow{1} \\
\stackrel{0}{-} \\
\dot{2} \\
\stackrel{1}{2} \\
\stackrel{1}{N}\end{array}$ & 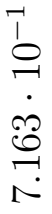 & 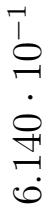 & $\begin{array}{l}1 \\
0 \\
0 \\
\dot{1} \\
2 \\
\infty \\
0 \\
\dot{0}\end{array}$ & 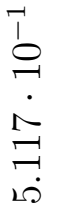 & $\begin{array}{l}\overrightarrow{1} \\
\stackrel{0}{-1} \\
\dot{\sim} \\
\stackrel{\sigma}{\sigma} \\
\dot{\gamma}\end{array}$ \\
\hline 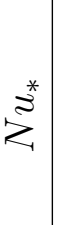 & $\begin{array}{l}\underset{i}{1} \\
\stackrel{-}{0} \\
\dot{N} \\
\stackrel{N}{\infty} \\
\dot{N}\end{array}$ & 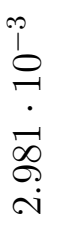 & 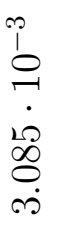 & $\begin{array}{l}\infty \\
\stackrel{p}{0} \\
\dot{1} \\
\dot{\infty} \\
\infty \\
\stackrel{\infty}{\infty}\end{array}$ & $\begin{array}{l}\infty \\
i \\
0 \\
\dot{0} \\
\dot{y} \\
\dot{N}\end{array}$ & $\begin{array}{l}q \\
i \\
\dot{\sigma} \\
\dot{0} \\
\dot{0} \\
\dot{+}\end{array}$ & 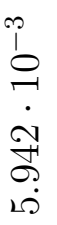 & $\begin{array}{l}\underset{j}{\rho} \\
\stackrel{-}{0} \\
\dot{\sigma} \\
\dot{m} \\
\dot{\sigma}\end{array}$ & 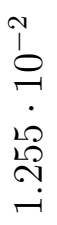 & 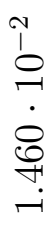 & 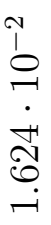 & 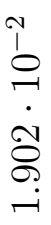 & 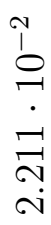 & 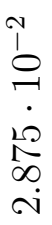 & 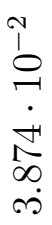 & 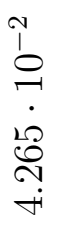 & 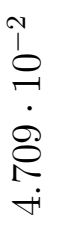 & $\begin{array}{l}N \\
1 \\
0 \\
\dot{1} \\
\infty \\
10 \\
10\end{array}$ \\
\hline$\underset{\underset{\sim}{\sim}}{\stackrel{*}{*}}$ & $\begin{array}{l}\stackrel{+}{1} \\
\ominus \\
\stackrel{-}{0} \\
\infty \\
\infty \\
\infty \\
\infty\end{array}$ & $\begin{array}{l}+1 \\
\stackrel{1}{0} \\
\stackrel{-1}{0} \\
\dot{\infty} \\
\dot{\infty}\end{array}$ & 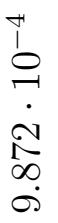 & $\begin{array}{l}\infty \\
\stackrel{\rho}{0} \\
\dot{\sigma} \\
\dot{\infty} \\
\infty \\
\dot{0}\end{array}$ & $\begin{array}{l}\infty \\
\dot{1} \\
\dot{-} \\
\dot{\sigma} \\
\dot{\sigma} \\
\overrightarrow{-}\end{array}$ & $\begin{array}{l}p \\
i \\
\stackrel{0}{0} \\
\stackrel{N}{N} \\
\underset{\sim}{*}\end{array}$ & 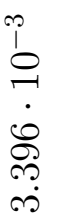 & 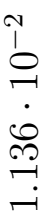 & 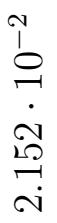 & 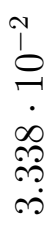 & 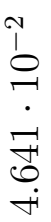 & 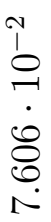 & 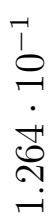 & 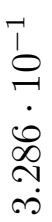 & $\begin{array}{l}8 \\
\stackrel{0}{-} \\
\dot{1} \\
\stackrel{0}{0} \\
\stackrel{-}{-}\end{array}$ & 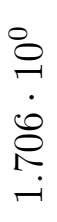 & 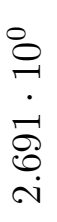 & $\begin{array}{l}\stackrel{O}{\sigma} \\
\dot{0} \\
\dot{\infty} \\
\dot{+} \\
\dot{0}\end{array}$ \\
\hline 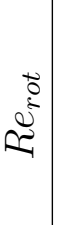 & 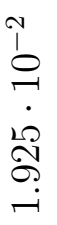 & $\begin{array}{l}\tilde{1} \\
\stackrel{0}{-1} \\
\dot{0} \\
\stackrel{10}{\infty} \\
\infty \\
\dot{+}\end{array}$ & 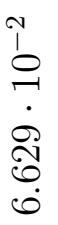 & 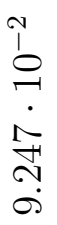 & $\begin{array}{l}\overrightarrow{1} \\
\stackrel{0}{-1} \\
\dot{0} \\
\stackrel{\leftrightarrow}{\longrightarrow} \\
\stackrel{\sim}{-1}\end{array}$ & 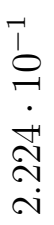 & 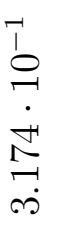 & 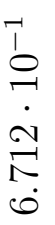 & $\begin{array}{l}1 \\
\varrho \\
\dot{\infty} \\
\infty \\
N \\
\infty \\
\infty\end{array}$ & 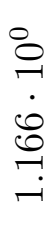 & 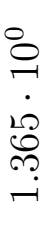 & 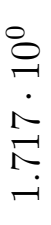 & 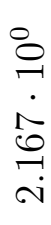 & 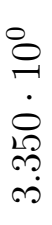 & 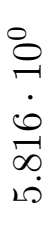 & 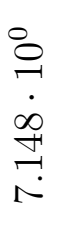 & $\begin{array}{l}\stackrel{\Xi}{\Xi} \\
\dot{\sim} \\
\underset{+}{+} \\
\infty \\
\infty\end{array}$ & 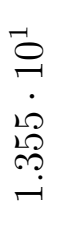 \\
\hline $\begin{array}{c}\overrightarrow{0} \\
\hat{j} \\
\hat{D}^{2}\end{array}$ & $\begin{array}{l}\vec{b} \\
\stackrel{-}{\circ} \\
\dot{\infty} \\
\underset{+}{+} \\
\stackrel{-}{-}\end{array}$ & $\begin{array}{l}\overrightarrow{1} \\
0 \\
\dot{1} \\
\dot{2} \\
\dot{\Omega} \\
\dot{m}\end{array}$ & $\begin{array}{l}\vec{\jmath} \\
\varrho \\
\dot{\jmath} \\
\dot{\bullet} \\
\stackrel{+}{0} \\
\dot{\nabla}\end{array}$ & $\begin{array}{l}\overrightarrow{1} \\
\stackrel{0}{1} \\
\dot{2} \\
\stackrel{N}{+} \\
\dot{0}\end{array}$ & 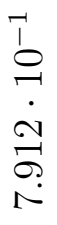 & 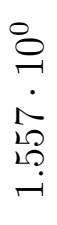 & $\begin{array}{l}\stackrel{0}{\Xi} \\
\dot{\sim} \\
\stackrel{N}{N} \\
\stackrel{\sim}{v}\end{array}$ & $\begin{array}{l}\mathscr{O} \\
\dot{1} \\
\dot{\infty} \\
0 \\
\dot{0} \\
\dot{+}\end{array}$ & $\begin{array}{l}8 \\
0 \\
0 \\
10 \\
0 \\
0 \\
0\end{array}$ & $\begin{array}{l}8 \\
\stackrel{1}{1} \\
\dot{1} \\
0 \\
\dot{0} \\
\infty\end{array}$ & 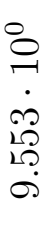 & 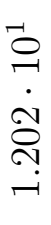 & 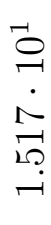 & 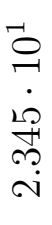 & 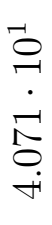 & 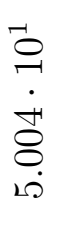 & $\begin{array}{l}\overrightarrow{0} \\
\dot{r} \\
\dot{m} \\
\dot{\sigma} \\
\dot{0}\end{array}$ & $\begin{array}{l}\overrightarrow{0} \\
\dot{1} \\
\dot{+} \\
\infty \\
\dot{+} \\
\dot{0}\end{array}$ \\
\hline$\sim$ & 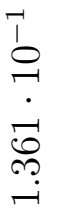 & 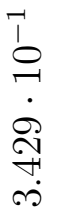 & $\begin{array}{l}\vec{\jmath} \\
\stackrel{1}{ } \\
\dot{1} \\
\dot{N} \\
\infty \\
0 \\
\dot{\theta}\end{array}$ & 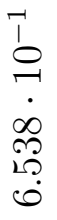 & 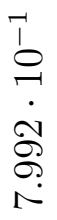 & 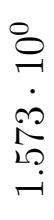 & 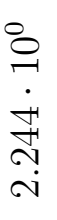 & 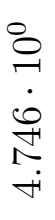 & $\begin{array}{l}\stackrel{0}{0} \\
\ddot{1} \\
\dot{0} \\
0 \\
0\end{array}$ & $\begin{array}{l}\stackrel{D}{O} \\
\dot{1} \\
\dot{\infty} \\
\stackrel{+}{N} \\
\infty\end{array}$ & 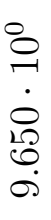 & 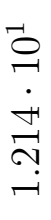 & 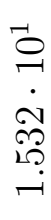 & 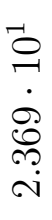 & 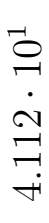 & 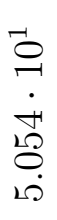 & $\begin{array}{l}\overrightarrow{0} \\
\ddots \\
\dot{0} \\
0 \\
0 \\
0 \\
0\end{array}$ & $\begin{array}{l}\overrightarrow{0} \\
\ddot{7} \\
\dot{\infty} \\
\infty \\
\dot{0} \\
\dot{0}\end{array}$ \\
\hline$\stackrel{2}{2}$ & 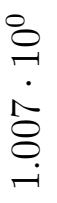 & 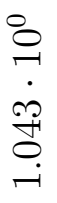 & 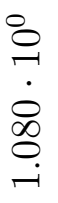 & 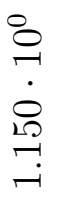 & $\begin{array}{l}\stackrel{\ominus}{\ominus} \\
\dot{N} \\
\stackrel{\sim}{\sim}\end{array}$ & 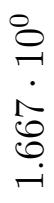 & 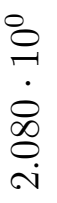 & $\begin{array}{l}\stackrel{D}{\varrho} \\
\dot{1} \\
\infty \\
\stackrel{N}{\sim} \\
\dot{\sim}\end{array}$ & 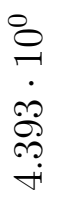 & 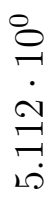 & $\begin{array}{l}\mathscr{8} \\
\stackrel{1}{1} \\
\dot{1} \\
\infty \\
0 \\
0 \\
\dot{0}\end{array}$ & $\begin{array}{l}8 \\
0 \\
\dot{1} \\
\dot{1} \\
0 \\
0 \\
0 \\
0\end{array}$ & $\begin{array}{l}\stackrel{8}{0} \\
\stackrel{1}{1} \\
\dot{2} \\
\stackrel{N}{N}\end{array}$ & 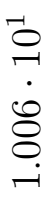 & 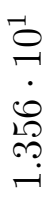 & $\begin{array}{l}\overrightarrow{0} \\
\dot{\sim} \\
\dot{\sim} \\
\ddot{\sim}\end{array}$ & 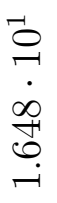 & 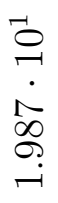 \\
\hline $\begin{array}{l}z^{2} \\
x \\
z^{2} \\
x \\
z^{8}\end{array}$ & 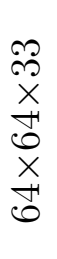 & 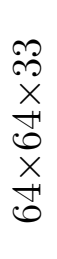 & 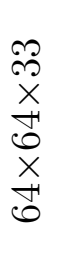 & $\begin{array}{l}m \\
x \\
x \\
\overrightarrow{0} \\
x \\
\overrightarrow{0}\end{array}$ & $\begin{array}{l}\text { ले } \\
\times \\
\vec{H} \\
x \\
\overrightarrow{4}\end{array}$ & $\begin{array}{l}\text { ñ } \\
x \\
\vec{y} \\
x \\
\vec{x}\end{array}$ & $\begin{array}{l}9 \\
x \\
x \\
0 \\
x \\
\dot{+} \\
0\end{array}$ & $\begin{array}{l}2 \\
x \\
x \\
\overrightarrow{0} \\
x \\
\overrightarrow{+}\end{array}$ & $\begin{array}{l}2 \\
x \\
x \\
\dot{0} \\
x \\
\overrightarrow{0}\end{array}$ & 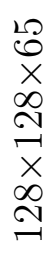 & 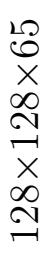 & 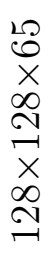 & $\begin{array}{l}\mathcal{L} \\
\times \\
\times \\
\infty \\
\sim \\
\sim \\
\times \\
\times \\
\infty \\
\stackrel{\sim}{N}\end{array}$ & $\begin{array}{l}10 \\
0 \\
\times \\
\infty \\
\sim \\
\sim \\
\times \\
\times \\
\infty \\
\stackrel{\sim}{\sim}\end{array}$ & 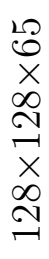 & $\begin{array}{l}0 \\
0 \\
\times \\
0 \\
10 \\
0 \\
\times \\
0 \\
10 \\
01\end{array}$ & $\begin{array}{l}10 \\
0 \\
x \\
0 \\
10 \\
0 \\
x \\
0 \\
10 \\
0\end{array}$ & $\begin{array}{c}10 \\
0 \\
\times \\
0 \\
10 \\
0 \\
\times \\
0 \\
10 \\
\end{array}$ \\
\hline$\approx$ & $\begin{array}{l}\underset{\sim}{\ominus} \\
\dot{+} \\
\dot{\nabla} \\
\dot{0}\end{array}$ & $\begin{array}{l}\stackrel{0}{\ominus} \\
\dot{0} \\
\dot{1} \\
\dot{1}\end{array}$ & $\begin{array}{l}\mathscr{\theta} \\
\stackrel{0}{0} \\
\dot{0} \\
\dot{0}\end{array}$ & $\begin{array}{l}\stackrel{\infty}{\ominus} \\
\dot{\infty} \\
\dot{\infty} \\
\dot{0}\end{array}$ & $\begin{array}{l}\stackrel{0}{\ominus} \\
\dot{0} \\
\dot{0}\end{array}$ & $\begin{array}{l}\stackrel{\infty}{\ominus} \\
\dot{0} \\
\dot{\infty}\end{array}$ & 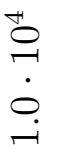 & $\begin{array}{l}\stackrel{+}{0} \\
\stackrel{-1}{0} \\
\dot{0} \\
\dot{v}\end{array}$ & $\begin{array}{l}\underset{0}{0} \\
\ddot{1} \\
\dot{0} \\
\dot{\infty}\end{array}$ & $\begin{array}{l}\underset{+}{0} \\
\dot{-1} \\
\dot{0}\end{array}$ & $\begin{array}{l}\stackrel{+}{\ominus} \\
\stackrel{1}{1} \\
\dot{0} \\
\dot{0}\end{array}$ & $\begin{array}{l}\stackrel{+}{ٍ} \\
\stackrel{-}{\circ} \\
\dot{\sigma}\end{array}$ & $\begin{array}{l}\stackrel{20}{\varrho} \\
\dot{0} \\
\dot{0}\end{array}$ & $\begin{array}{l}\stackrel{0}{\varrho} \\
\dot{-1} \\
\dot{0} \\
\dot{0}\end{array}$ & $\begin{array}{l}\stackrel{\ominus}{\ominus} \\
\stackrel{1}{\circ} \\
\dot{0} \\
\dot{0}\end{array}$ & $\begin{array}{l}\stackrel{10}{\ominus} \\
\stackrel{1}{0} \\
\dot{N}\end{array}$ & $\begin{array}{l}\stackrel{0}{0} \\
\stackrel{1}{\prime} \\
\dot{0} \\
\dot{\sim}\end{array}$ & $\begin{array}{l}\stackrel{0}{0} \\
\dot{-1} \\
\dot{0} \\
\dot{v}\end{array}$ \\
\hline
\end{tabular}




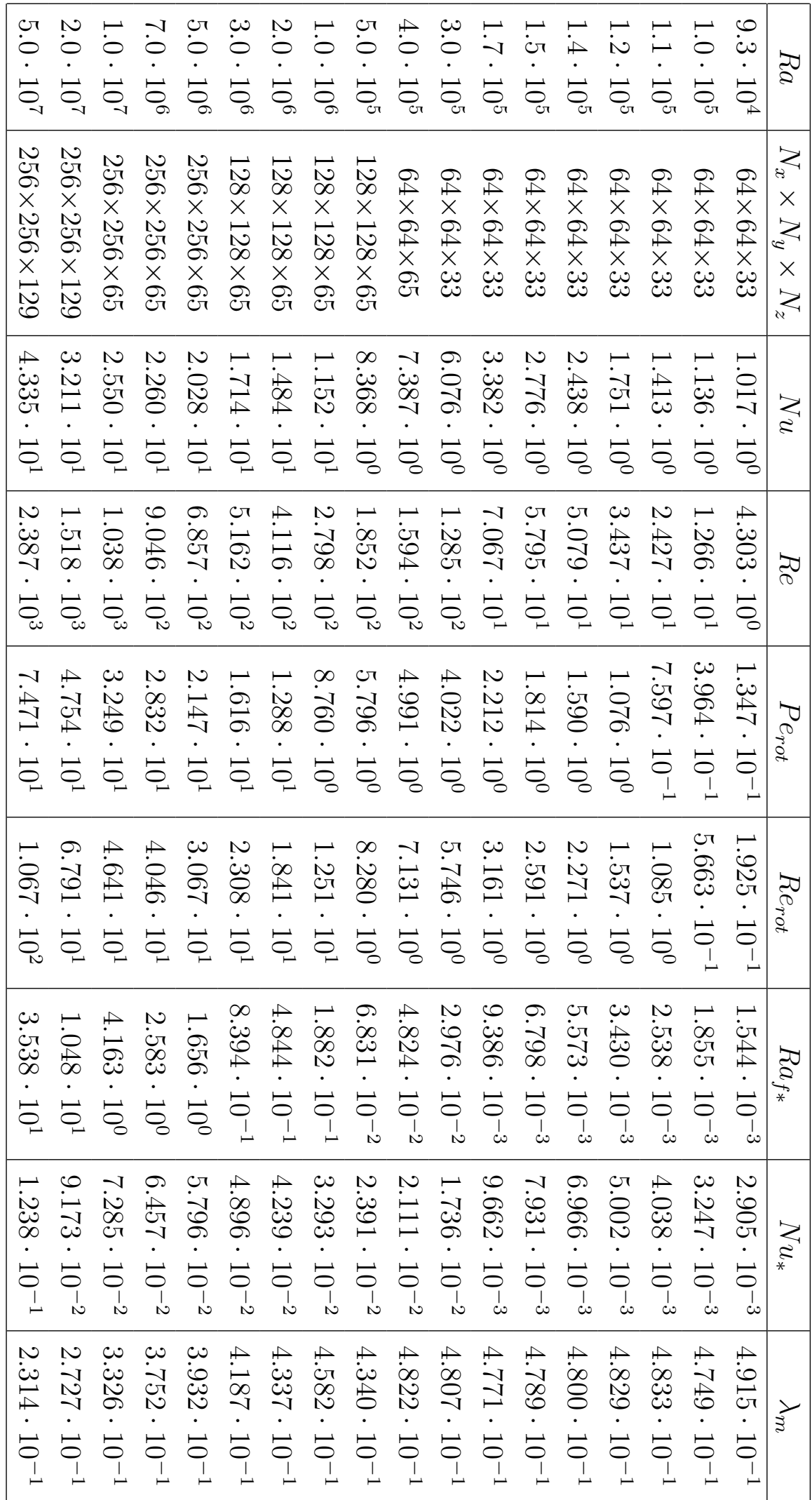




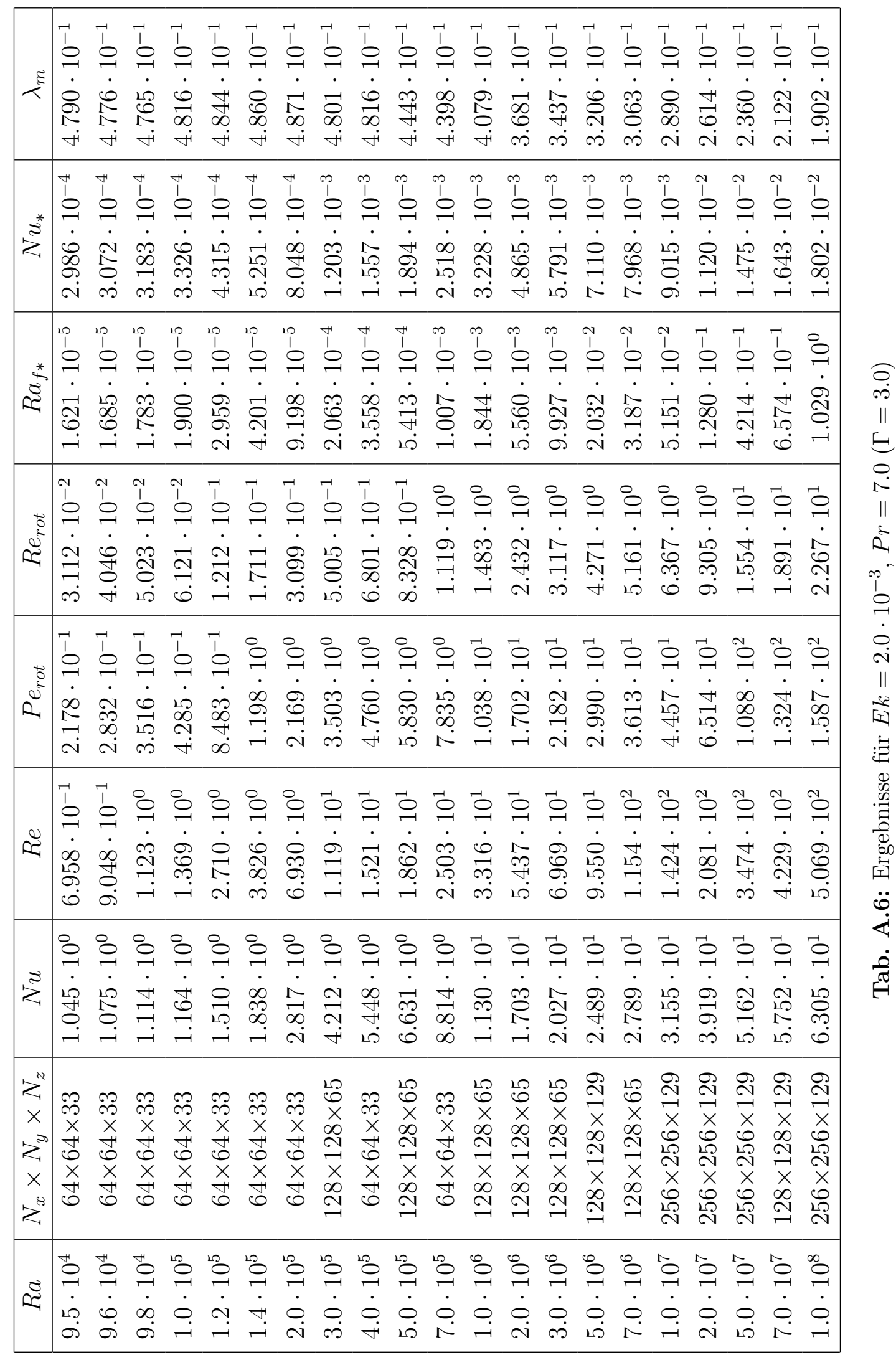


cr

0 o cr 0 o 0 o 0 o ir 0 o it iv 0 o

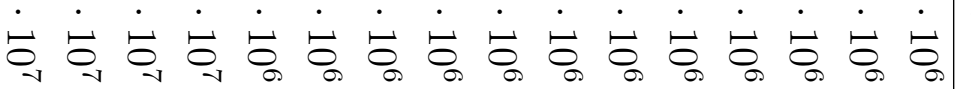

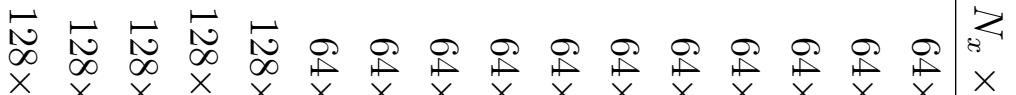

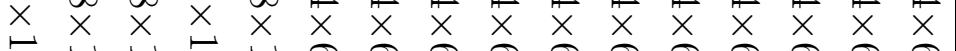

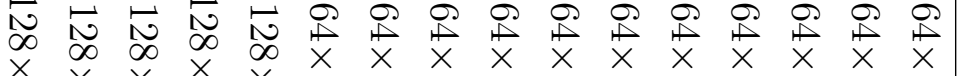

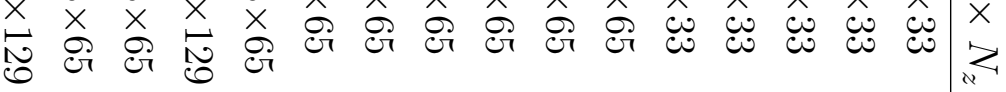

ஸि 焉

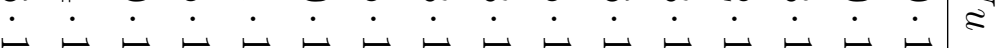

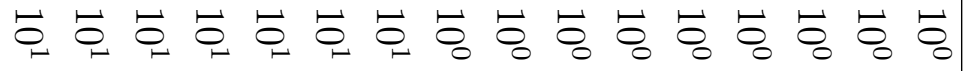
$\vdash$ ○ ש (4)

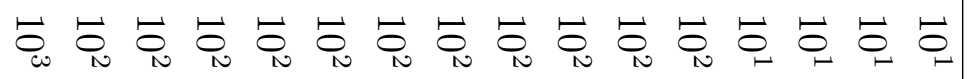

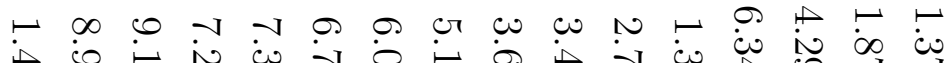

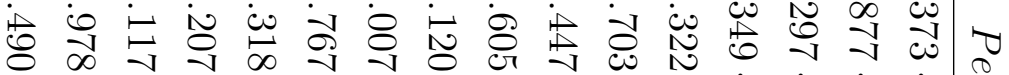
$\dot{\sigma} \dot{0} \dot{0} \dot{0} \overrightarrow{0} \dot{0} \dot{0} \dot{0} \dot{0} \dot{0} \dot{0} \dot{0} \overrightarrow{0} \dot{0} \dot{0} \dot{0}$

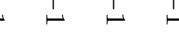

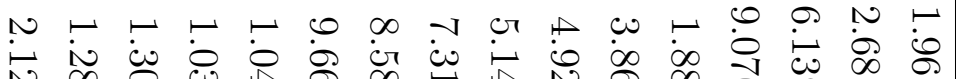

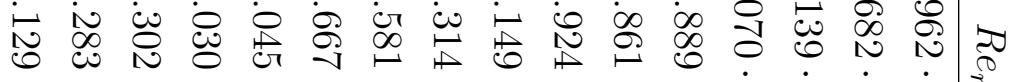

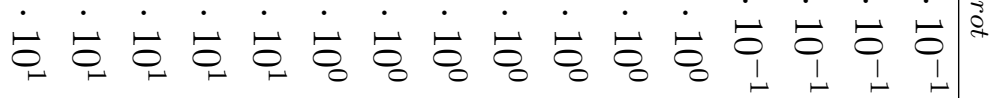

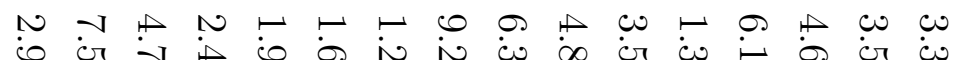

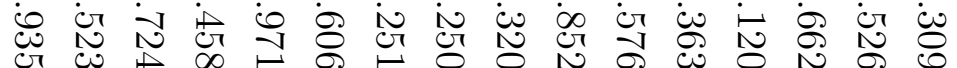

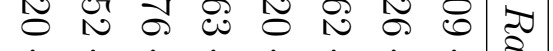

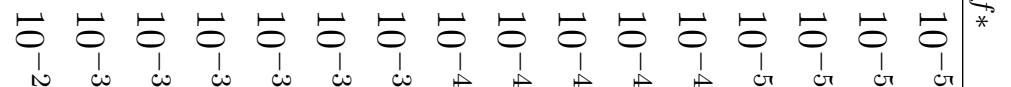

$\vdash$ ○ î

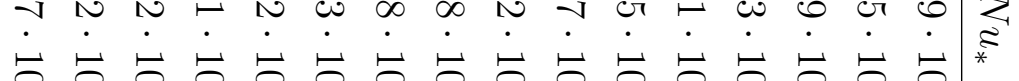

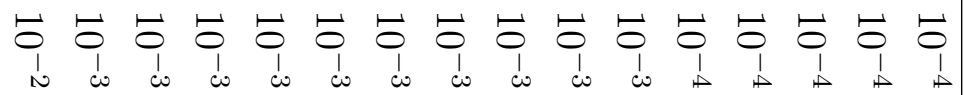

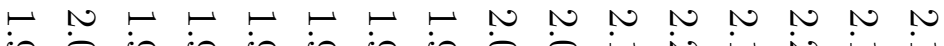

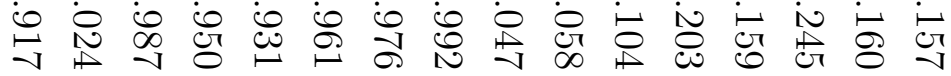

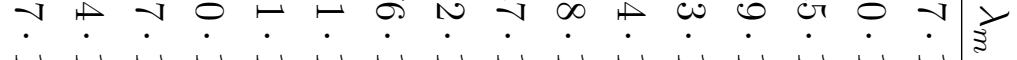

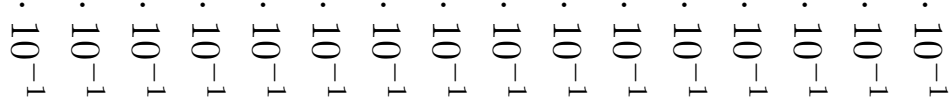




\begin{tabular}{|c|c|c|c|c|c|c|c|c|c|}
\hline ह & 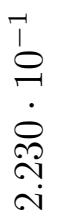 & 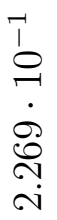 & $\begin{array}{l}\overrightarrow{1} \\
\stackrel{0}{ } \\
\dot{1} \\
\stackrel{1}{N} \\
\stackrel{\sim}{\sim}\end{array}$ & 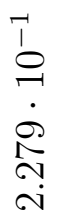 & 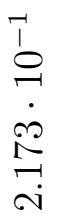 & 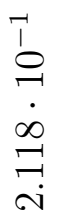 & 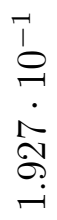 & 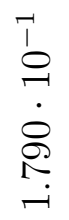 & 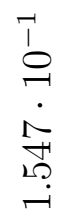 \\
\hline$\sum_{2}^{\stackrel{*}{*}}$ & 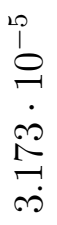 & 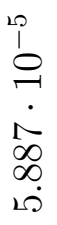 & $\begin{array}{l}1 \\
1 \\
0 \\
\dot{1} \\
\dot{0} \\
\dot{\infty}\end{array}$ & 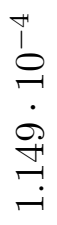 & 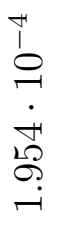 & $\begin{array}{l}+1 \\
\stackrel{+}{0} \\
\dot{1} \\
\dot{2} \\
\dot{\sim} \\
\dot{\sim}\end{array}$ & 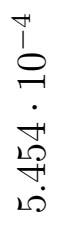 & 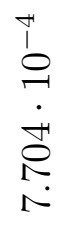 & 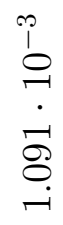 \\
\hline$\underset{\sim}{\stackrel{*}{*}}$ & 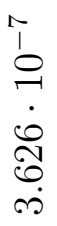 & 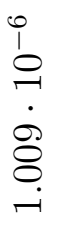 & \begin{tabular}{l}
0 \\
1 \\
0 \\
$\dot{1}$ \\
$\sim$ \\
$\infty$ \\
0 \\
\hdashline
\end{tabular} & $\begin{array}{l}0 \\
1 \\
0 \\
\dot{1} \\
\dot{\infty} \\
\stackrel{\infty}{\sim} \\
\dot{m}\end{array}$ & $\begin{array}{l}0 \\
1 \\
0 \\
\dot{1} \\
\dot{N} \\
\infty \\
\infty\end{array}$ & 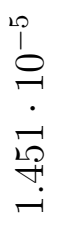 & 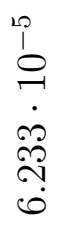 & 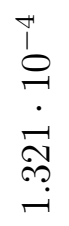 & 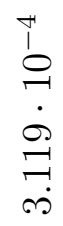 \\
\hline & $\begin{array}{l}\uparrow \\
1 \\
0 \\
1 \\
\dot{1} \\
0 \\
0 \\
\infty \\
\infty\end{array}$ & 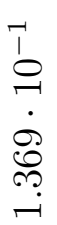 & 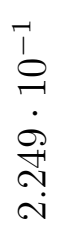 & 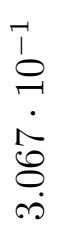 & 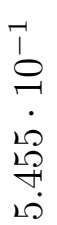 & 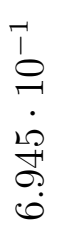 & 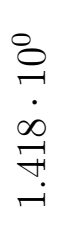 & $\begin{array}{l}0 \\
\ominus \\
\dot{\sigma} \\
0 \\
0 \\
\dot{0}\end{array}$ & 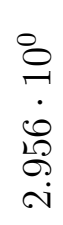 \\
\hline $\begin{array}{c}\overrightarrow{0} \\
\hat{D}^{\hat{L}}\end{array}$ & 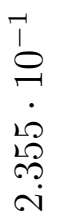 & 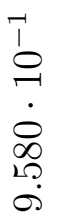 & 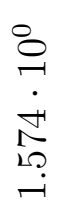 & 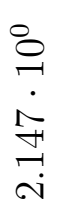 & $\begin{array}{l}\stackrel{0}{\ominus} \\
\dot{\sim} \\
\dot{\infty} \\
\infty \\
\dot{\infty}\end{array}$ & \begin{tabular}{l}
$\circlearrowright$ \\
\hdashline \\
$\dot{\ddots}$ \\
$\dot{0}$ \\
$\infty$ \\
$\dot{+}$
\end{tabular} & 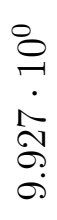 & 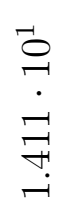 & $\begin{array}{l}\overrightarrow{0} \\
\dot{1} \\
\dot{0} \\
0 \\
0 \\
ن\end{array}$ \\
\hline$\sim$ & 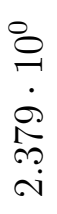 & 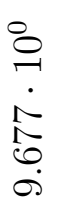 & 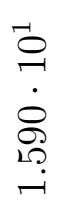 & 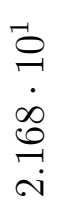 & $\begin{array}{l}\overrightarrow{0} \\
\\
\dot{1} \\
10 \\
\infty \\
\infty \\
\dot{0}\end{array}$ & 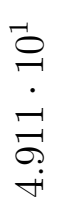 & 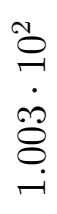 & 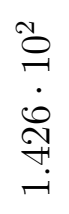 & 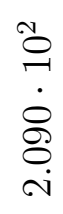 \\
\hline$\stackrel{\overrightarrow{2}}{z_{1}}$ & 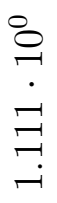 & 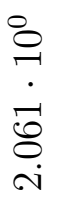 & 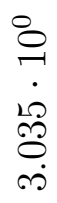 & 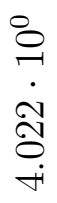 & $\begin{array}{l}\stackrel{0}{\varrho} \\
\dot{\Omega} \\
\dot{\infty} \\
\infty \\
\dot{0}\end{array}$ & $\begin{array}{l}\stackrel{0}{\varrho} \\
\dot{1} \\
\infty \\
\infty \\
\infty \\
\infty\end{array}$ & 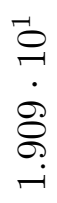 & 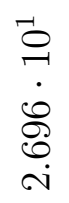 & 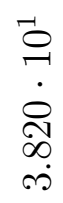 \\
\hline $\begin{array}{l}z_{1}^{N} \\
x \\
z_{1}^{2} \\
x \\
z^{*}\end{array}$ & 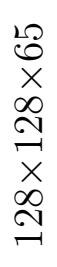 & $\begin{array}{l}0 \\
0 \\
\times \\
\infty \\
\sim \\
-1 \\
\times \\
\infty \\
\sim \\
\sim \\
\sim\end{array}$ & 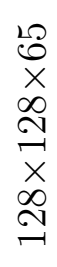 & 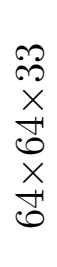 & 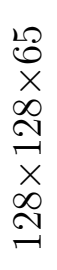 & 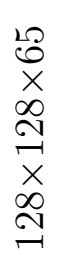 & 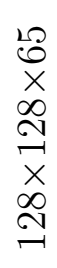 & 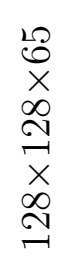 & 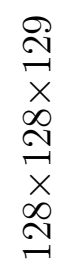 \\
\hline$\sim$ & $\begin{array}{l}\stackrel{0}{\varrho} \\
\neg \\
\dot{0} \\
\dot{0}\end{array}$ & $\begin{array}{l}\stackrel{0}{0} \\
\stackrel{1}{0} \\
\dot{0} \\
\dot{\infty}\end{array}$ & $\begin{array}{l}\stackrel{\ominus}{\ominus} \\
\dot{\bullet} \\
\dot{\nabla}\end{array}$ & $\begin{array}{l}\stackrel{0}{0} \\
\ddot{\ddots} \\
\dot{0} \\
\dot{0}\end{array}$ & $\begin{array}{l}\stackrel{0}{\varrho} \\
\underset{1}{0} \\
\dot{0} \\
\dot{\infty}\end{array}$ & 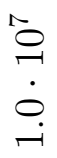 & $\begin{array}{l}\stackrel{0}{ت} \\
\dot{0} \\
\dot{0}\end{array}$ & 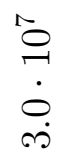 & $\begin{array}{l}\stackrel{0}{7} \\
\dot{0} \\
\dot{0}\end{array}$ \\
\hline
\end{tabular}




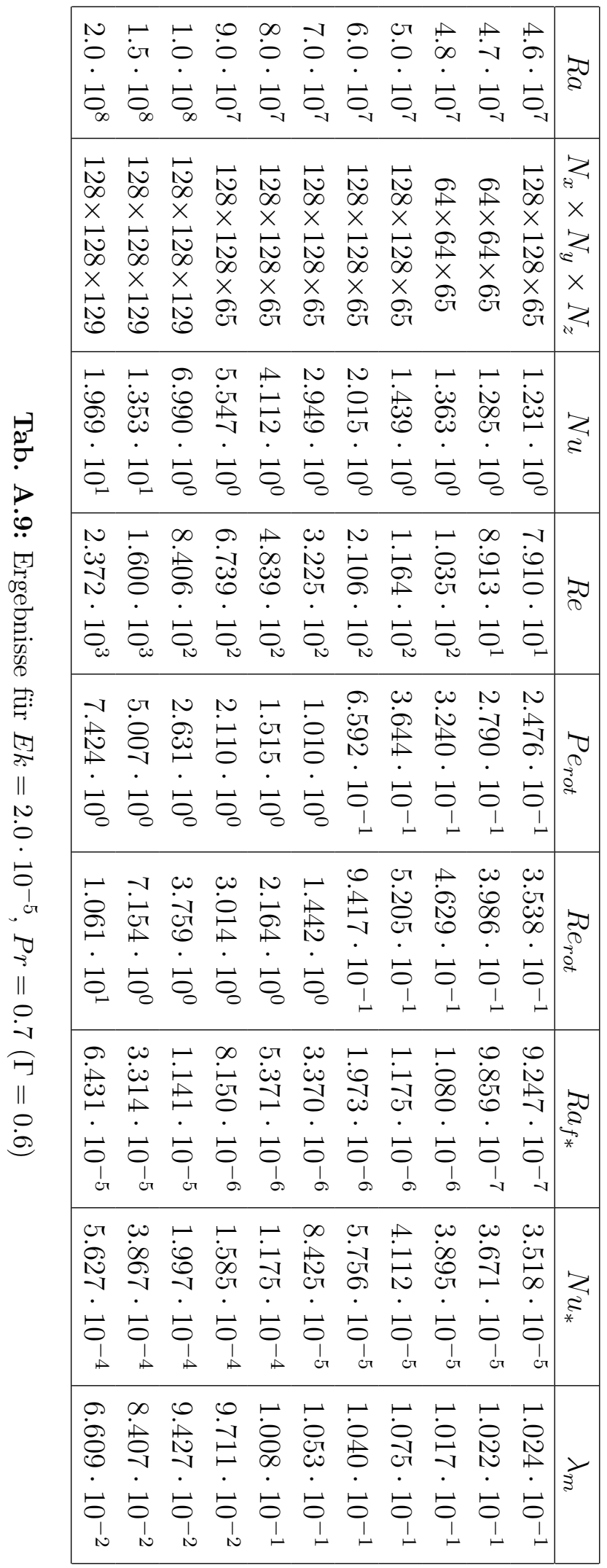




\section{Anhang B}

\section{Chebychev-Polynome}

In dieser Arbeit werden zur Diskretisierung der Variablen in z-Richtung Chebychev-Polynome verwendet. Hier sollen kurz deren Eigenschaften und nützliche Relationen (siehe z.B. [3], [4]) vorgestellt werden.

Die Chebychev-Polynome sind die Lösungen der Differentialgleichung

$$
\left(1-x^{2}\right) \partial_{x}^{2} T_{n}(x)-x \partial_{x} T_{n}(x)+n^{2} T_{n}(x)=0
$$

Das Chebychev-Polynom n-ten Grades $T_{n}(x)$ kann angegeben werden als:

$$
\begin{aligned}
& T_{n}(x)=\cos (n \arccos (x)) \\
& T_{n}(x)=\frac{1}{2}\left[\left(x+\sqrt{x^{2}-1}\right)^{n}+\left(x-\sqrt{x^{2}-1}\right)^{n}\right]
\end{aligned}
$$

Der Definitionsbereich liegt ebenso wie der Wertebereich zwischen -1 und 1. Durch Umformungen ([4]) erhält man eine Rekursionsformel für $n \geq 2$ :

$$
T_{n}(x)=2 x T_{n-1}(x)-T_{n-2}(x)
$$

Daraus ergeben sich die Werte für die ersten 6 Polynome:

$$
\begin{aligned}
& T_{0}(x)=1 \\
& T_{1}(x)=x \\
& T_{2}(x)=2 x^{2}-1 \\
& T_{3}(x)=4 x^{3}-3 x \\
& T_{4}(x)=8 x^{4}-8 x^{2}+1 \\
& T_{5}(x)=16 x^{5}-20 x^{3}+5 x
\end{aligned}
$$




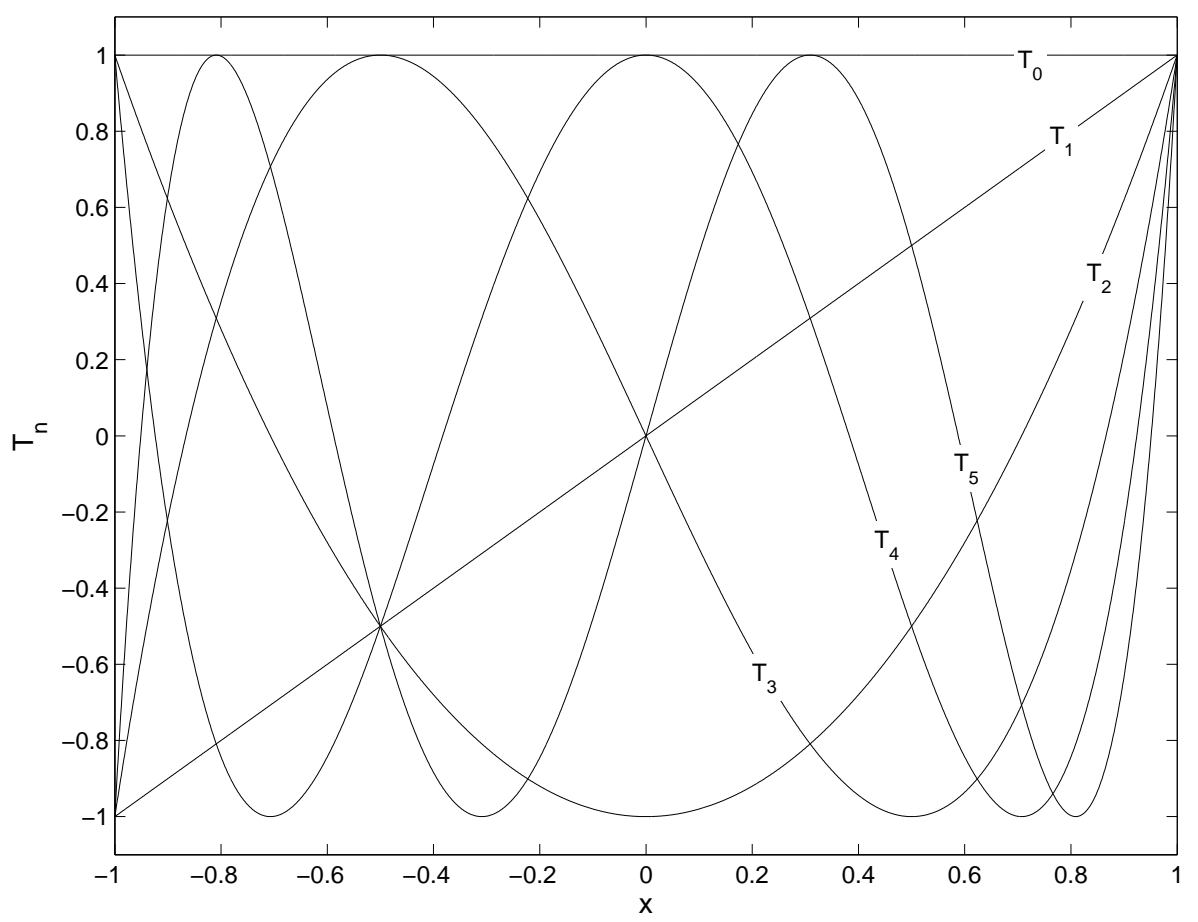

Abb. B.1: Verlauf der ersten 6 Chebychev-Polynome

Deren Verlauf ist in B.1 dargestellt:

Mit den folgenden Formeln ist es möglich, den Wert der Chebychev-Polynome und deren Ableitungen am Rand zu berechnen:

$$
\begin{aligned}
T_{n}( \pm 1) & =( \pm 1)^{n} \\
\partial_{x} T_{n}( \pm 1) & =n^{2}( \pm 1)^{n+1} \\
\partial_{x}^{2} T_{n}( \pm 1) & =n^{2} \frac{n^{2}-1}{3}( \pm 1)^{n} \\
\partial_{x}^{3} T_{n}( \pm 1) & =n^{2} \frac{n^{2}-1}{3} \frac{n^{2}-4}{5}( \pm 1)^{n+1}
\end{aligned}
$$

Diese werden benötigt, um die Randbedingungen in den Zeitschritt-Matrizen (siehe das Beispiel in Abschnitt 3.3.1) zu setzen. Die Randwerte $x=\tilde{z}= \pm 1$ in den Formeln entsprechen wegen Gleichung 3.17 gerade den gewünschten Werten $z=0$ und $z=1$. 


\section{Anhang C}

\section{Kinetische Energiedissipation}

In diesem Kapitel soll eine Beziehung für die kinetische Energiedissipation $\epsilon_{u}$ hergeleitet werden. Dabei wird von den Gleichungen (2.1a, 2.1b, 2.1c) ausgegangen, die in ihrer dimensionslosen Form so aussehen:

$$
\begin{gathered}
\partial_{t} \mathbf{u}+(\mathbf{u} \cdot \nabla) \mathbf{u}+2 \frac{\operatorname{Pr}}{E k}(\hat{\mathbf{z}} \times \mathbf{u})=-\nabla p+\operatorname{Pr} \Delta \mathbf{u}+\operatorname{Ra} \operatorname{Pr} T \hat{\mathbf{z}} \\
\partial_{t} T+(\mathbf{u} \cdot \nabla) T=\Delta T \\
\nabla \cdot \mathbf{u}=0
\end{gathered}
$$

Es werden folgende Konventionen im Simulationsgebiet verwendet:

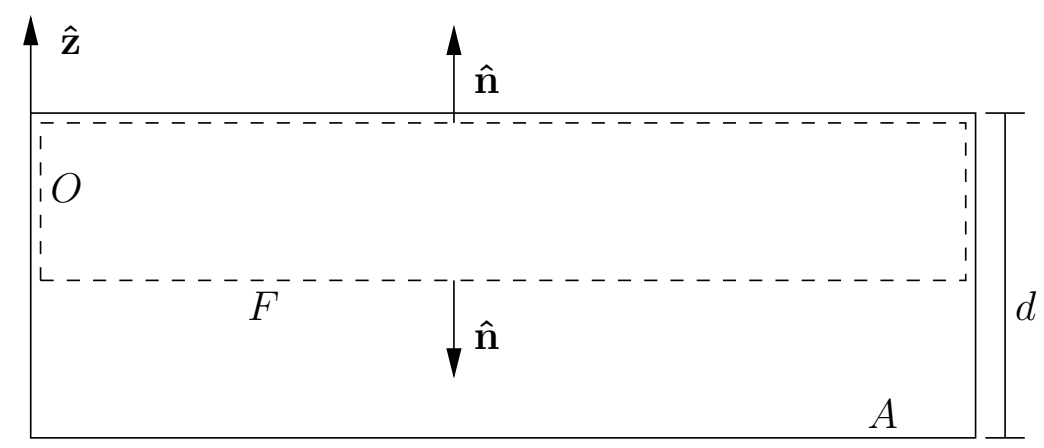

Abb. C.1: Schematische Darstellung des Simulationsgebiets mit den verwendeten Konventionen.

Es ist

- F eine beliebige horizontale Fläche 
- $O$ eine geschlossene Fläche, die $F$, den oberen Rand und die Seitenränder enthält

- $\hat{\mathbf{n}}$ der nach außen gerichtete Normalenvektor zu $O$

- $\hat{\mathbf{z}}$ der nach oben gerichtete Einheitsvektor

- $d$ die Zellhöhe

- $A$ die Zellquerschnittsfläche

Die Temperaturen sind oben und unten vorgegeben, wie in 2.1. Die Temperatur ist die Summe aus einer statischen Temperaturverteilung $T_{s}$ und einem Wechselanteil $\theta$ :

$$
T=T_{s}+\theta \quad \text { mit } \quad \frac{d T_{s}}{d z}=\frac{T_{2}-T_{1}}{d}=-\frac{\Delta T}{d}(\text { nach } 2.3)
$$

Zuerst wird C.1 mit u multipliziert und über das gesamte Volumen integriert. Danach wird das zeitliche Mittel gebildet:

$$
\begin{aligned}
\underbrace{\int \partial_{t}\left(\frac{1}{2} \mathbf{u}^{2}\right) d V}_{1}+\underbrace{\int \mathbf{u}[(\mathbf{u} \cdot \nabla) \mathbf{u}] d V}_{2}+2 \frac{\operatorname{Pr}}{E k} \underbrace{\int \mathbf{u}(\hat{\mathbf{z}} \times \mathbf{u}) d V}_{3}= \\
-\underbrace{\int \mathbf{u}(\nabla p) d V}_{4}+\operatorname{Pr} \underbrace{\int \mathbf{u}\left(\nabla^{2} \mathbf{u}\right) d V}_{5}+\operatorname{Ra} \operatorname{Pr} \underbrace{\int \mathbf{u}(T \hat{\mathbf{z}}) d V}_{6}
\end{aligned}
$$

Term 1 ist im zeitlichen Mittel Null:

$$
\int\left\langle\partial_{t}\left(\frac{1}{2} \mathbf{u}^{2}\right) d V\right\rangle_{t}=0
$$

Term 2 ist in Indexschreibweise:

$$
\begin{aligned}
\int u_{i} u_{j} \partial_{j} u_{i} d V & =\int u_{i} \partial_{j}\left(u_{j} u_{i}\right) d V \quad\left(\text { wegen } \partial_{j} u_{j}=0\right) \\
& =\int\left[\partial_{j}\left(u_{i} u_{j} u_{i}\right)-u_{j} u_{i} \partial_{j} u_{i}\right] d V \\
& =\oint_{O} n_{j} u_{i} u_{j} u_{i} d S-\int u_{j} u_{i} \partial_{j} u_{i} d V \quad \text { (Satz von Gauss) }
\end{aligned}
$$

Das Oberflächenintegral ist Null, da $u_{j} n_{j}=0$ ist am oberen/unteren Rand und periodische Ränder verwendet werden. Es bleibt:

$$
\int u_{i} u_{j} \partial_{j} u_{i} d V=-\int u_{j} u_{i} \partial_{j} u_{i} d V
$$


Dies ist nur erfüllt, wenn gilt:

$$
\int u_{i} u_{j} \partial_{j} u_{i} d V=0
$$

Term 3 ist:

$$
\int \mathbf{u}(\hat{\mathbf{z}} \times \mathbf{u}) d V=0
$$

Dies gilt, da $\mathbf{u} \perp(\hat{\mathbf{z}} \times \mathbf{u})$

Term 4 ist:

$$
\begin{aligned}
\int \mathbf{u}(\nabla p) d V & =\int \nabla(\mathbf{u} p) d V \quad(\text { wegen } \nabla \mathbf{u}=0) \\
& =\oint_{O} \hat{\mathbf{n}} \cdot \mathbf{u} p d S
\end{aligned}
$$

Dieses Oberflächenintegral ist Null am oberen/unteren Rand (wegen $\mathbf{u} \perp \hat{\mathbf{n}}$ ) und wegen der periodischen Randbedingungen für die Geschwindigkeit. Letzteres gilt nur in Verbindung mit einem periodischen Druck $p$. Ist der Druck hingegen

$$
p=p_{s}+\tilde{p} \quad \text { mit } \tilde{p} \text { periodisch, } \nabla p_{s}=\hat{\mathbf{z}} \frac{d p_{s}}{d z}
$$

dann ist

$$
\begin{aligned}
\int \mathbf{u}(\nabla p) d V & =\int \mathbf{u}\left(\nabla p_{s}\right) d V+\int \mathbf{u}(\nabla \tilde{p}) d V \\
& =\frac{d p_{s}}{d z} \int \mathbf{u} \cdot \hat{\mathbf{z}} d V+0 \\
& =\frac{d p_{s}}{d z} \int d z \int u_{z} d S=0 \quad \text { (wegen Inkompressibilität) }
\end{aligned}
$$

Term 5 ist in Indexschreibweise:

$$
\begin{aligned}
\int u_{i} \partial_{j} \partial_{j} u_{i} d V & =\int\left[\partial_{j}\left(u_{i} \partial_{j} u_{i}\right)-\left(\partial_{j} u_{i}\right)\left(\partial_{j} u_{i}\right)\right] d V \\
& =\oint_{O} n_{j}\left(u_{i} \partial_{j} u_{i}\right) d S-\int\left(\partial_{j} u_{i}\right)\left(\partial_{j} u_{i}\right) d V
\end{aligned}
$$

Das Oberflächenintegral ist wieder Null, da im schubspannungsfreien Fall an der oberen/unteren Wand $\partial_{j} u_{i}=0$ gilt.

Es bleibt also von C.5 übrig, wenn man noch die zeitliche Mittelung anwendet:

$$
\begin{gathered}
0=\left\langle-\operatorname{Pr} \int\left(\partial_{j} u_{i}\right)\left(\partial_{j} u_{i}\right) d V+\operatorname{Ra} \operatorname{Pr} \int \mathbf{u} \cdot(T \hat{\mathbf{z}}) d V\right\rangle_{t} \\
\Longrightarrow \int\left\langle\left(\partial_{j} u_{i}\right)\left(\partial_{j} u_{i}\right)\right\rangle_{t} d V=\operatorname{Ra} \int\left\langle u_{z} T\right\rangle_{t} d V
\end{gathered}
$$


Anhang C. Kinetische Energiedissipation

Die linke Seite ist gerade die kinetische Energiedissipation:

$$
\epsilon_{u} V=\int\left\langle\left(\partial_{j} u_{i}\right)\left(\partial_{j} u_{i}\right)\right\rangle_{t} d V
$$

Die rechte Seite lässt sich ersetzen mithilfe C.2. Dazu wird diese zeitlich gemittelt und integriert:

$$
\begin{array}{rlrl}
\int\left\langle\partial_{t} T\right\rangle_{t} d V & =\int\left\langle-(\mathbf{u} \cdot \nabla) T+\nabla^{2} T\right\rangle_{t} d V & \\
\Longrightarrow 0 & =\int \nabla\left[-\langle\mathbf{u} T\rangle_{t}+\langle\nabla T\rangle_{t}\right] d V \quad & \text { (wegen Inkompressibilität) } \\
& =\oint_{O}\left[-\langle\mathbf{u} T\rangle_{t}+\langle\nabla T\rangle_{t}\right] \hat{\mathbf{n}} d S \quad \text { (Satz von Gauss) }
\end{array}
$$

Die horizontalen Ränder liefern wegen der Periodizität wieder keinen Beitrag. Es bleibt:

$$
\int_{F}\left[-\langle\mathbf{u} T\rangle_{t}+\langle\nabla T\rangle_{t}\right](-\hat{\mathbf{z}}) d S+\int_{\text {oben }}\langle\nabla T\rangle_{t} \hat{\mathbf{z}} d S=0
$$

Das rechte Integral ist gerade der Wärmefluss durch den oberen Rand, also

$$
\begin{gathered}
\int_{\text {oben }}\langle\nabla T\rangle_{t} \hat{\mathbf{z}} d S=A N u\left(\frac{d T_{s}}{d z}\right) \\
\Longrightarrow \int_{F}\left[-\langle\mathbf{u} T\rangle_{t}+\langle\nabla T\rangle_{t}\right] \hat{\mathbf{z}} d S=A N u \frac{d T_{s}}{d z}
\end{gathered}
$$

Diese Gleichung integriert man nun über $z$ :

$$
-\int\langle\mathbf{u} T\rangle_{t} \hat{\mathbf{z}} d V+\int_{F} d S \int \partial_{z}\langle T\rangle_{t} d z=A N u \frac{d T_{s}}{d z} d
$$

Mit $\int \partial_{z}\langle T\rangle_{t} d z=T_{2}-T_{1}=d \frac{d T_{s}}{d z}$ (nach C.4) folgt:

$$
\begin{aligned}
-\int\langle\mathbf{u} T\rangle_{t} \hat{\mathbf{z}} d V & =A N u \frac{d T_{s}}{d z} d-\int_{F} d S d \frac{d T_{s}}{d z} \\
& =A N u \frac{d T_{s}}{d z} d-A d \frac{d T_{s}}{d z} \\
& =V \frac{d T_{s}}{d z}(N u-1) \\
\Longrightarrow \int\langle\mathbf{u} T\rangle_{t} \hat{\mathbf{z}} d V & =-V \frac{d T_{s}}{d z}(N u-1)=V(N u-1)
\end{aligned}
$$

Hierbei wurde ausgenutzt, dass für das entdimensionalisierte statische Temperaturprofil gilt:

$$
\frac{d T_{s}}{d z}=-1
$$

Dieses Ergebnis setzt man nun in C.6 ein und erhält nach Kürzen von $V$ :

$$
\epsilon_{u}=R a(N u-1)
$$




\section{Literaturverzeichnis}

[1] http://www.catea.gatech.edu/grade/mecheng/mod8/mod8.html 1, 103

[2] http://eol.jsc.nasa.gov/sseop/images/ISD/lowres/STS037/STS037-72-29. JPG 1, 103

[3] Abramowitz, Milton (Hrsg.) ; Stegun, Irene A. (Hrsg.): Handbook of Mathematical Functions with Formulas, Graphs, and Mathematical Tables. New York: Dover, 1972. 771-802 S. 91

[4] Arfken, George B.: Mathematical methods for physicists. Academic Press, 1985. - 731748 S. 91

[5] Aurnou, J. M.: Planetary core dynamics and convective heat transfer scaling. In: Geophysical and Astrophysical Fluid Dynamics 101 (2007), Oktober, S. 327-345. http: //dx.doi.org/10.1080/03091920701472568. - DOI 10.1080/03091920701472568 11, 56, $57,76,105,108$

[6] BÉnARd, Henri: Les tourbillons cellulaires dans une nappe liquide. - Méthodes optiques d'observation et d'enregistrement. In: J. Phys. Theor. Appl. 10 (1901), Nr. 1, 254266. http://dx.doi.org/10.1051/jphystap:0190100100025400. - DOI 10.1051/jphystap:0190100100025400 2

[7] Busse, F. H.: Non-linear properties of thermal convection. In: Reports on Progress in Physics 41 (1978), Dezember, S. 1929-1967. http://dx.doi.org/10.1088/0034-4885/ 41/12/003. - DOI 10.1088/0034-4885/41/12/003 7

[8] Buzbee, Bill: The SLATEC Common Math Library. In: Cowell, Wayne R. (Hrsg.): Sources and development of mathematical software. Prentice-Hall, Inc., 1984. - ISBN 0-13-823501-5 20 
[9] Castaing, Bernard ; Gunaratne, Gemunu ; Heslot, François ; Kadanoff, Leo ; Libchaber, Albert ; Thomae, Stefan ; Wu, Xiao-Zhong ; Zaleski, Stéphane ; Zanetti, Gianluigi: Scaling of hard thermal turbulence in Rayleigh-Bénard convection. In: Journal of Fluid Mechanics Digital Archive 204 (1989), Nr. -1, S. 1-30. http://dx.doi.org/10. 1017/S0022112089001643. - DOI 10.1017/S0022112089001643 39, 40

[10] Chandrasekhar, S.: Hydrodynamic and Hydromagnetic Stability. Oxford University Press, 1961 12, 14, 58, 103, 105, 108

[11] Chavanne, X. ; Chillà, F. ; Chabaud, B. ; Castaing, B. ; Hébral, B.: Turbulent Rayleigh-Bénard convection in gaseous and liquid He. In: Physics of Fluids 13 (2001), Nr. 5, 1300-1320. http://dx.doi.org/10.1063/1.1355683. - DOI 10.1063/1.1355683 39

[12] Christensen, U. R.: Zonal flow driven by strongly supercritical convection in rotating spherical shells. In: Journal of Fluid Mechanics 470 (2002), Nr. -1, S. 115-133. http: //dx.doi.org/10.1017/S0022112002002008. - DOI 10.1017/S0022112002002008 54, 55

[13] Cioni, S. ; Ciliberto, S. ; Sommeria, J.: Strongly turbulent Rayleigh-Bénard convection in mercury: comparism with results at moderate Prandtl number. In: Journal of Fluid Mechanics 335 (1997), Nr. -1, S. 111-140 41

[14] Ferziger, J. H. ; Peric, M.: Computational Methods for Fluid Dynamics. Springer, 2002 21

[15] Goldstein, R. J. ; Chiang, H. D. ; See, D. L.: High-Rayleigh-number convection in a horizontal enclosure. In: Journal of Fluid Mechanics Digital Archive 213 (1990), Nr. -1, S. 111-126. http://dx.doi.org/10.1017/S0022112090002245. - DOI $10.1017 /$ S0022112090002245 41

[16] Greenspan, Harvey P.: The theory of rotating fluids. Cambridge Univ. Press, 196937

[17] Gropp, W. ; Lusk, E. ; SkJellum, A.: MPI- eine Einführung: Portable parallele Programmierung mit dem Message-passing Interface. Verlag Oldenbourg Wissenschaftsverlag, 200731

[18] Grossmann, S. ; Lohse, D.: Scaling in thermal convection: a unifying theory. In: Journal of Fluid Mechanics 407 (2000), Nr. -1, S. 27-56. http://dx.doi.org/10.1017/ S0022112099007545. - DOI 10.1017/S0022112099007545 39, 41, 60 
[19] HartleP, Thomas: Strukturbildung und Turbulenz. Eine numerische Studie zur turbulenten Rayleigh-Benard Konvektion. In: Dissertation, Mathematisch-Naturwissenschaftliche Fakultät, Universität Göttingen (2004). http://webdoc.sub.gwdg.de/diss/2005/ hartlep/ 19, 27, 49, 79

[20] Heslot, F. ; Castaing, B. ; Libchaber, A.: Transitions to turbulence in helium gas. In: Phys. Rev. A 36 (1987), Dec, Nr. 12, S. 5870-5873. http://dx.doi.org/10.1103/ PhysRevA.36.5870. - DOI 10.1103/PhysRevA.36.5870 41, 42

[21] HIDE, R.: The viscous boundary layer at the free surface of a rotating baroclinic fluid. In: Tellus A 16 (1964), S. 523-529 37

[22] Horanyi, S. ; Krebs, L. ; MÜller, U.: Turbulent Rayleigh-Bénard convection in low Prandtl-number fluids. In: International Journal of Heat and Mass Transfer 42 (1999), Nr. 21, 3983 - 4003. http://dx.doi.org/DOI:10.1016/S0017-9310 (99)00059-9. - DOI DOI: 10.1016/S0017-9310(99)00059-9. - ISSN 0017-9310 41

[23] Julien, K. ; LegG, S. ; McWilliams, J. ; Werne, J.: Hard turbulence in rotating Rayleigh-Bénard convection. In: Phys. Rev. E 53 (1996), Jun, Nr. 6, S. R5557-R5560. http://dx.doi.org/10.1103/PhysRevE.53.R5557. - DOI 10.1103/PhysRevE.53.R5557 53

[24] King, Eric M. ; Stellmach, Stephan ; Noir, Jerome ; Hansen, Ulrich ; Aurnou, Jonathan M.: Boundary layer control of rotating convection systems. In: Nature 457 (2009), S. 301-304. http://dx.doi.org/10.1038/nature07647. - DOI 10.1038/nature07647 45, 53

[25] King, Eric M. ; Stellmach, Stephan ; Noir, Jerome ; Hansen, Ulrich ; Aurnou, Jonathan M.: Supplementary material for: Boundary layer control of rotating convection systems. In: Nature 457 (2009), 301-304. http://dx.doi.org/10.1038/nature07647. DOI $10.1038 /$ nature 0764745,53

[26] Kunnen, R. P. J. ; Clercx, H. J. H. ; Geurts, B. J.: Heat flux intensification by vortical flow localization in rotating convection. In: Physical Review E (Statistical, Nonlinear, and Soft Matter Physics) 74 (2006), Nr. 5, 056306. http://dx.doi.org/10.1103/PhysRevE. 74.056306. - DOI 10.1103/PhysRevE.74.056306 53

[27] Lagarias, Jeffrey C. ; Reeds, James A. ; Wright, Margaret H. ; Wright, Paul E.: Convergence Properties of the Nelder-Mead Simplex Method in Low Dimensions. In: 
SIAM Journal on Optimization 9 (1998), Nr. 1, S. 112-147. http://dx.doi.org/10. 1137/S1052623496303470. - DOI 10.1137/S1052623496303470. - ISSN 1052-6234 64

[28] Melchior, P.: The physics of the earth's core. erste Auflage. Pergamon Press, 198676

[29] Moser, R. D. ; Moin, P. ; Leonard, A.: A spectral numerical method for the NavierStokes equations with applications to Taylor-Couette flow. In: Journal of Computational Physics 52 (1983), Dezember, S. 524-544. http://dx.doi.org/10.1016/0021-9991(83) 90006-2. - DOI 10.1016/0021-9991(83)90006-2 27

[30] Nelder, J. A. ; Mead, R.: A Simplex Method for Function Minimization. In: The Computer Journal 7 (1965), Nr. 4, 308-313. http://dx.doi.org/10.1093/comjnl/7.4. 308. - DOI 10.1093/comjnl/7.4.308 64

[31] Oberbeck, A.: Über die Wärmeleitung der Flüssigkeiten bei Berücksichtigung der Strömungen infolge von Temperaturdifferenzen. In: Ann. Phys. Chem. 7 (1879), S. 271-292 7

[32] Press, William H.: Numerical Recipes in Fortran 77. Cambridge University Press, 1989 18,27

[33] Rossby, H. T.: A study of Bénard convection with and without rotation. In: Journal of Fluid Mechanics Digital Archive 36 (1969), Nr. 02, S. 309-335. http://dx.doi.org/10. 1017/S0022112069001674. - DOI 10.1017/S0022112069001674 2

[34] Schmitt, Burkhard J.; WAHL, Wolf von: Mean values and non-periodic pressure in convection problems between plates or with stress-free boundaries. In: Pacific Journal of Mathematics 185 (1998), Nr. 2, S. 347-362 15

[35] Schmitz, S. ; Tilgner, A.: Heat transport in rotating convection without Ekman layers. In: Physical Review E (Statistical, Nonlinear, and Soft Matter Physics) 80 (2009), Nr. 1, 015305. http://dx.doi.org/10.1103/PhysRevE.80.015305. - DOI 10.1103/PhysRevE. 80.0153053

[36] Schubert, G.: Treatise on Geophysics. Bd. 8. Amsterdam : Elsevier, 200777

[37] Shraiman, Boris I. ; Siggia, Eric D.: Heat transport in high-Rayleigh-number convection. In: Phys. Rev. A 42 (1990), Sep, Nr. 6, S. 3650-3653. http://dx.doi.org/10.1103/ PhysRevA.42.3650. - DOI 10.1103/PhysRevA.42.3650 41 
[38] Stellmach, Stephan ; Hansen, Ulrich: Cartesian convection driven dynamos at low Ekman number. In: Phys. Rev. E 70 (2004), Nov, Nr. 5, S. 056312. http://dx.doi.org/ 10.1103/PhysRevE.70.056312. - DOI 10.1103/PhysRevE.70.056312 57

[39] Vorobieff, Peter ; Ecke, Robert E.: Vortex structure in rotating Rayleigh-Bénard convection. In: Physica D: Nonlinear Phenomena 123 (1998), Nr. 1-4, 153 - 160. http: //dx.doi.org/DOI:10.1016/S0167-2789(98)00118-3. - DOI DOI: 10.1016/S01672789(98)00118-3. - ISSN 0167-2789 65

[40] Xin, Y.-B. ; XiA, K.-Q. ; Tong, P.: Measured Velocity Boundary Layers in Turbulent Convection. In: Phys. Rev. Lett. 77 (1996), Aug, Nr. 7, S. 1266-1269. http://dx.doi. org/10.1103/PhysRevLett.77.1266. - DOI 10.1103/PhysRevLett.77.1266 39

[41] Zhong, Jin-Qiang; Stevens, Richard J. A. M. ; Clercx, Herman J. H. ; Verzicco, Roberto ; Lohse, Detlef ; AhLERs, Guenter: Prandtl-, Rayleigh-, and Rossby-Number Dependence of Heat Transport in Turbulent Rotating Rayleigh-Bénard Convection. In: Physical Review Letters 102 (2009), Nr. 4, 044502. http://dx.doi.org/10.1103/PhysRevLett. 102.044502. - DOI 10.1103/PhysRevLett.102.044502 53 



\section{Abbildungsverzeichnis}

1.1 Konvektionszellen bei Rayleigh-Bénard-Konvektion. Links: Bénard-Zellen in einer dünnen Flüssigkeitsschicht (aus [1]). Rechts: Konvektionszellen über dem Pazifik (aus [2]) . . . . . . . . . . . . . . . . . . . . . . . . . . . . . . . . 1

2.1 Aufbau des Simulationsgebiets mit Abmessungen. Schematisch eingezeichnet sind zwei Konvektionsrollen. . . . . . . . . . . . . . . . . . . . . . . . . . . 6

2.2 Bestimmung des Onsets durch Linearisieren: Abkling- und Anwachsraten $\beta$ für $E k=\infty$ (links) und $E k=2 \cdot 10^{-3}$ (rechts) . . . . . . . . . . . . . . . 13

2.3 Vergleich zwischen Literatur (aus [10]) und linearisierten Simulationen. Ein linearer Fit für genügend kleine Ekman-Zahlen ist ebenfalls eingetragen. . . . . . 14

3.1 Links: Verteilung der Stützstellen für $N_{z}=65$ Chebychev-Polynome. Rechts: Vergrößerung des Randbereichs. . . . . . . . . . . . . . . . . . . 20

4.1 Aufbau des Programms: Flussdiagramm mit den wichtigsten Programmschritten. 29

4.2 Speicheraufteilung: Links das horizontale Speicherschema, bei der jeder Prozessor eine oder mehrere horizontale Ebenen mit allen Wellenzahlen k kennt. Die Daten liegen im Ortsraum vor, die „vergessene“ Ebene bei $N_{z}$ ist mit eingezeichnet. Rechts das vertikale Speicherschema mit den Daten im Spektralraum. . . . . . . 31

4.3 Dargestellt ist die Aufteilung der Berechnung der expliziten rechten Seiten, die teilweise im Spektral- und teilweise im Ortsraum durchgeführt wird. . . . . . . . 33 
5.1 Auftragung des Ebenenmittels der Horizontalgeschwindigkeit $\left\langle u_{h}\right\rangle_{x, y}$ über der Zellhöhe $z$ bei verschiedenen Rayleigh-Zahlen.

Links: No-slip Ränder oben und unten mit $E k=1.7 \cdot 10^{-3}$ und $\operatorname{Pr}=7.0$.

Rechts: Schubspannungsfreie Ränder oben und unten mit $E k=2.0 \cdot 10^{-3}$ und

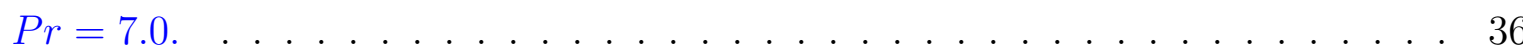

5.2 Auftragung der Grenzschichtdicke $\delta_{u}$ über der Rayleigh-Zahl (links) und normiert mit $E k^{0.486}$ über der Reynolds-Zahl (rechts) . . . . . . . . . . . . . . 37

5.3 Grenzschichtdicke $\delta_{u}$ für schubspannungsfreie Ränder, aufgetragen über der Rayleigh-Zahl. . . . . . . . . . . . . . . . . . . . . 38

5.4 Abhängigkeit der Reynolds-Zahl von der Rayleigh-Zahl ohne und mit Rotation

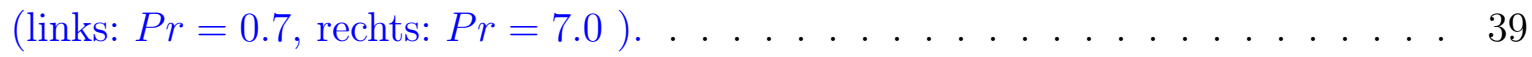

5.5 Abhängigkeit der Péclet-Zahl von der Rayleigh-Zahl mit und ohne Rotation. Zusätzlich eingetragen sind zwei Fitgeraden mit Steigung $\frac{1}{2}$. . . . . . . . . 40

5.6 Wärmetransport in Abhängigkeit der Rayleigh-Zahl für den Fall ohne Rotation.

5.7 Konvektiv transportierte Wärme $N u-1$ in Abhängigkeit der Rayleigh-Zahl für den Fall ohne Rotation. . . . . . . . . . . . . . . . . . . . . . . . 42

5.8 Konvektiv transportierte Wärme in Abhängigkeit der Péclet-Zahl für den Fall ohne Rotation. Gezeigt sind zusätzlich Ergebnisse von Simulationen mit no-slip Rändern oben und unten. . . . . . . . . . . . . . . . . . . 43

5.9 Wärmetransport in Abhängigkeit der Rayleigh-Zahl ohne und mit Rotation. . . 44

5.10 Konvektiv transportierte Wärme in Abhängigkeit der Rotations-Péclet-Zahl für Simulationen mit schubspannungsfreien Rändern oben und unten. . . . . . . . . 46

5.11 Konvektiv transportierte Wärme (wie in Abbildung 5.10), kompensiert mit $P e_{\text {rot }}^{2 / 3}$ (links) bzw. $P e_{\text {rot }}^{4 / 7}$ (rechts), aufgetragen über $P e_{\text {rot }} \ldots \ldots \ldots \ldots$. . . . 47

5.12 Konvektiv transportierte Wärme in Abhängigkeit der Rotations-Péclet-Zahl für Simulationen mit no-slip Rändern oben und unten. . . . . . . . . . . . . . 48

5.13 Auftragung des Ebenenmittel der Temperatur $\langle T\rangle_{x, y}$ über der Zellhöhe $z$ bei verschiedenen Rayleigh-Zahlen.

Links: No-slip Ränder oben und unten mit $E k=1.7 \cdot 10^{-3}$ und $\operatorname{Pr}=7.0$.

Rechts: Schubspannungsfreie Ränder oben und unten mit $E k=2.0 \cdot 10^{-3}$ und $\operatorname{Pr}=7.0$. 
5.14 Gradient der horizontal gemittelten Temperatur $\partial_{z}\langle T\rangle_{x, y}$ bei $z=0.5$ in der Zellmitte, aufgetragen über $\left(R a-R a_{c}\right) / R a_{c} \ldots \ldots \ldots \ldots \ldots$

5.15 Thermische Grenzschichtdicke $\delta_{T}$ über der Rayleigh-Zahl Ra . . . . . . . . . 51

5.16 Verhältnis $N u(\Omega) / N u(0)$ in Abhängigkeit der Rotations-Péclet-Zahl. Ein „Überschießen" wie im no-slip Fall ist nicht zu erkennen. . . . . . . . . . . . . . . . 51

5.17 Isoflächen der Temperatur für $\operatorname{Pr}=0.7$ (linke Spalte) und $\operatorname{Pr}=7.0$ (rechte Spalte).

Oben: $E k=2 \cdot 10^{-2}, R a=1 \cdot 10^{5}, T=0.75 \Delta T$ (rot) und $T=0.25 \Delta T$ (blau).

Mitte: $E k=2 \cdot 10^{-3}, R a=1 \cdot 10^{6}, T=0.7 \Delta T$ (rot) und $T=0.3 \Delta T$ (blau).

Unten: $E k=2 \cdot 10^{-4}, R a=1 \cdot 10^{7}, T=0.65 \Delta T$ (rot) und $T=0.35 \Delta T$ (blau). . 52

5.18 Auftragung der diffusionsfreien Kennzahlen $N u_{*}$ über $R a_{f *}$ für alle Simulationen mit schubspannungsfreien Rändern aus dem Bereich $0.5 \leq P e_{\text {rot }} \leq 10 \ldots \ldots$. . . 55

5.19 Vergleich der diffusionsfreien Kennzahlen dieser Arbeit (blaue Quadrate) mit den Ergebnissen anderer Veröffentlichungen (Original entnommen aus [5]) . . . . . . 56

5.20 Auftragung der diffusionsfreien Kennzahlen $N u_{*}$ über $R a_{f *}$ für alle Simulationen mit no-slip Rändern aus dem Bereich $0.5 \leq P e_{\text {rot }} \leq 10 \ldots \ldots \ldots$. . . . . 56

5.21 Schraubenbewegung der Fluidteilchen unter dem Einfluss der Corioliskraft: Beispiel einer Teilchenbahn aus der Literatur (links, siehe [10]) und aus einer Simu-

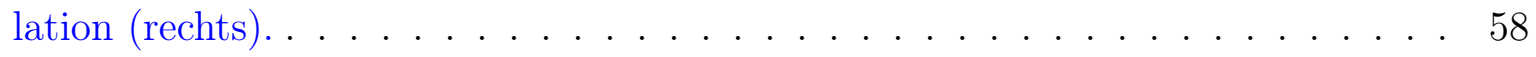

5.22 Ebenenmittel der Helizität aufgetragen über der Zellhöhe für sechs verschiedene Rayleigh-Zahlen bei konstanter Ekman- und Prandtl-Zahl $\left(E k=2 \cdot 10^{-3}, \operatorname{Pr}=\right.$

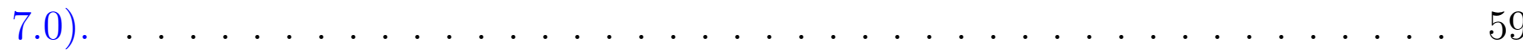

5.23 Auftragung des rms-Werts der Helizität $H_{r m s}$ über $P e_{\text {rot }}$ (links) und $R e_{\text {rot }}$ (rechts) für Simulationen mit Rotation. . . . . . . . . . . . . . . . . 6 60

5.24 Kinetische Energiedissipation $\epsilon_{u}$ für Simulationen ohne Rotation, normiert mit $\operatorname{Re}^{3} \operatorname{Pr}^{2}$, aufgetragen über der Reynolds-Zahl Re. . . . . . . . . . . 61

5.25 Kinetische Energiedissipation $\epsilon_{u}$ für Simulationen mit Rotation, normiert mit dem rotationsfreien Verhalten $R e^{3} P r^{2}$, aufgetragen über der RotationsReynolds-Zahl $R e_{r o t} \ldots \ldots \ldots \ldots \ldots \ldots$

5.26 Spektren des konvektiven Anteils $u_{z} T$ des Wärmestroms in der Mittelebene bei $z=0.5$ (links: spektrale Verteilung $V(k, z)$, rechts: spektrale Dichte $D(\lambda, z)$ ) einer Simulation mit $R a=1 \cdot 10^{6}, \operatorname{Pr}=0.7$ und $E k=\infty \ldots \ldots 62$ 
5.27 Spektrale Dichte des konvektiven Wärmetransports $u_{z} T$, analog zu Abbildung 5.26. Die Schnitte sind hier bei den Höhen $z=0.164$ (links) und $z=0.015$

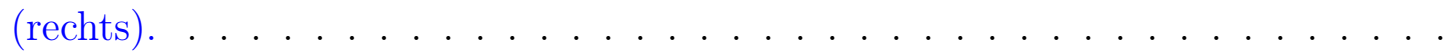

5.28 Fit an die spektralen Dichte mithilfe einer Gaussglocke nach Gleichung (5.11). Eingezeichnet ist zusätzlich die gesuchte Wellenlänge $\lambda_{m} \ldots \ldots \ldots$. . . . . 64

5.29 Auftragung der repräsentativen Wellenlänge $\lambda_{m}$ bei $z=0.5$ in der Mitte der Fluidschicht. Eingezeichnet sind Fits für den Bereich des Onsets und den nichtrotierenden Bereich. . . . . . . . . . . . . . . . . . . . . .

5.30 Vergleichende Spektren einer Simulation mit den Paramtern $E k=2 \cdot 10^{-3}, R a=$ $5 \cdot 10^{7}, \operatorname{Pr}=0.7, \operatorname{Re}_{\text {rot }} \approx 106$ bei $\Gamma=3.0$ (links) und $\Gamma=6.0$ (rechts) . . . . 66

5.31 Spektrale Dichte des Wärmetransports für $E k=\infty$ bei $z=0.5$ mit $R a=1 \cdot 10^{5}$ (obere Zeile), $R a=7 \cdot 10^{5}$ (mittlere Zeile) und $R a=1 \cdot 10^{6}$ (untere Zeile). Die Prandtl-Zahlen sind $\operatorname{Pr}=0.7$ links und $\operatorname{Pr}=7.0$ rechts. . . . . . . . . . 68

5.32 Schnitte des konvektiven Wärmetransports für $E k=\infty$ bei $z=0.5$ mit $R a=$ $1 \cdot 10^{5}$ (obere Zeile), $R a=7 \cdot 10^{5}$ (mittlere Zeile) und $R a=1 \cdot 10^{6}$ (untere Zeile). Die Prandtl-Zahlen sind $\operatorname{Pr}=0.7$ links und $\operatorname{Pr}=7.0$ rechts. . . . . . . . . . . 69

5.33 Spektrale Dichte des Wärmetransports für $E k=2 \cdot 10^{-2}$ bei $z=0.5$ mit $R a=$ $1 \cdot 10^{5}$ (obere Zeile), $R a=1 \cdot 10^{6}$ (mittlere Zeile) und $R a=5 \cdot 10^{6}$ (untere Zeile). Die Prandtl-Zahlen sind $\operatorname{Pr}=0.7$ links und $\operatorname{Pr}=7.0$ rechts. . . . . . . . . . 70

5.34 Schnitte des konvektiven Wärmetransports für $E k=2 \cdot 10^{-2}$ bei $z=0.5 \mathrm{mit}$ $R a=1 \cdot 10^{5}$ (obere Zeile), $R a=1 \cdot 10^{6}$ (mittlere Zeile) und $R a=5 \cdot 10^{6}$ (untere Zeile). Die Prandtl-Zahlen sind $\operatorname{Pr}=0.7$ links und $\operatorname{Pr}=7.0$ rechts. . . . . . . 71

5.35 Spektrale Dichte des Wärmetransports für $E k=2 \cdot 10^{-3}$ bei $z=0.5$ mit $R a=$ $1 \cdot 10^{6}$ (obere Zeile), $R a=1 \cdot 10^{7}$ (mittlere Zeile) und $R a=5 \cdot 10^{7}$ (untere Zeile). Die Prandtl-Zahlen sind $\operatorname{Pr}=0.7$ links und $\operatorname{Pr}=7.0$ rechts. . . . . . . . . . . 72

5.36 Schnitte des konvektiven Wärmetransports für $E k=2 \cdot 10^{-3}$ bei $z=0.5$ mit $R a=1 \cdot 10^{6}$ (obere Zeile), $R a=1 \cdot 10^{7}$ (mittlere Zeile) und $R a=5 \cdot 10^{7}$ (untere Zeile). Die Prandtl-Zahlen sind $\operatorname{Pr}=0.7$ links und $\operatorname{Pr}=7.0$ rechts. . . . . . . 73

5.37 Spektrale Dichte des Wärmetransports für $E k=2 \cdot 10^{-4}$ bei $z=0.5$ mit $R a=$ $5 \cdot 10^{6}$ (obere Zeile), $R a=1 \cdot 10^{7}$ (mittlere Zeile) und $R a=5 \cdot 10^{7}$ (untere Zeile). Die Prandtl-Zahlen sind $\operatorname{Pr}=0.7$ links und $\operatorname{Pr}=7.0$ rechts. . . . . . . . . . 74 
5.38 Schnitte des konvektiven Wärmetransports für $E k=2 \cdot 10^{-4}$ bei $z=0.5$ mit $R a=1 \cdot 10^{6}$ (obere Zeile), $R a=5 \cdot 10^{7}$ (mittlere Zeile) und $R a=5 \cdot 10^{7}$ (untere Zeile). Die Prandtl-Zahlen sind $\operatorname{Pr}=0.7$ links und $\operatorname{Pr}=7.0$ rechts. . . . . . . 75

B.1 Verlauf der ersten 6 Chebychev-Polynome . . . . . . . . . . . . . . . . . 92

C.1 Schematische Darstellung des Simulationsgebiets mit den verwendeten Konventionen. . . . . . . . . . . . . . . . . . . . . . . 93 


\section{Tabellenverzeichnis}

2.1 Gegnüberstellung der Kennzahlen des Erdkerns (aus [5]) und der Simulationen dieser Arbeit. . . . . . . . . . . . . . . . . . . . . 11

2.2 Vergleich der kritischen Rayleigh-Zahlen $R a_{c}$ für verschiedene Randbedingungen. 12

2.3 Vergleich der kritischen Rayleigh-Zahl $R a_{c}$ bzw. der kritischen Wellenlänge $\lambda_{c}$ für verschiedene Ekman-Zahlen. Die Literaturwerte sind aus [10] entnommen. . . 14

A.1 Ergebnisse für $E k=\infty, \operatorname{Pr}=0.7(\Gamma=10.0) \ldots \ldots \ldots$. . . . . . 82

A.2 Ergebnisse für $E k=\infty, \operatorname{Pr}=7.0(\Gamma=10.0) \ldots \ldots . \ldots . \ldots . . \ldots 3$

A.3 Ergebnisse für $E k=2.0 \cdot 10^{-2}, P r=0.7(\Gamma=5.0) \ldots \ldots$. . . . . . . . . 84

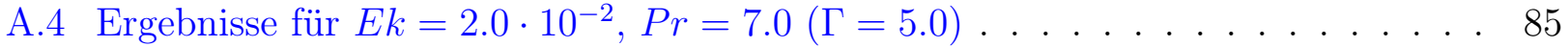

A.5 Ergebnisse für $E k=2.0 \cdot 10^{-3}, \operatorname{Pr}=0.7(\Gamma=3.0) \ldots \ldots$. . . . . . . . . 86

A.6 Ergebnisse für $E k=2.0 \cdot 10^{-3}, \operatorname{Pr}=7.0(\Gamma=3.0) \ldots \ldots$. . . . . . . . . 87

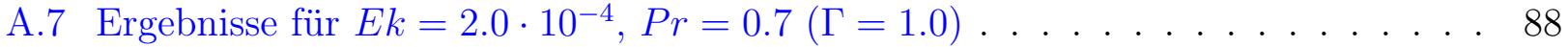

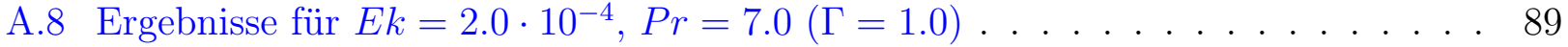

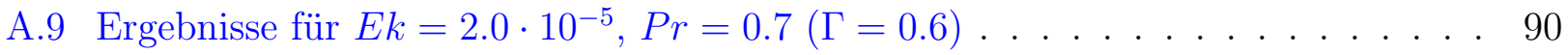




\section{Danksagung}

Mein besonderer Dank gilt Herrn Prof. Dr. A. Tilgner für seine gute Betreuung bei meiner Promotion. Seine Unterstützung durch immerwährende Diskussionsbereitschaft und Anregungen hat mir sehr geholfen.

Danken möchte ich auch Herrn Prof. Dr. U. Christensen für die Übernahme des Korreferats. Allen jetzigen und ehemaligen Arbeitsgruppenmitgliedern, insbesondere Arno Ickler, möchte ich für die nette Arbeitsatmosphäre danken.

Und schließlich danke ich auch meiner Frau Ellen, die mich während der gesamten Promotion begleitet und unterstützt hat: Danke für Alles! 



\section{Lebenslauf}

Name:

geboren:

Staatsangehörigkeit:

Familienstand:

seit $02 / 2005$

$11 / 2004$

$11 / 2002-11 / 2004$

$03 / 2001-11 / 2002$

$09 / 2000-02 / 2001$

$09 / 1998-08 / 2000$

08/1997-08/1998

$05 / 1997$

$1990-1997$

$1986-1990$
Simon Schmitz

17. August 1977 in Bad Neustadt a. d. Saale

deutsch

verheiratet, 2 Kinder

Anstellung am Geophysikalischen Institut als wissenschaftlicher Angestellter und Promotion

Diplomprüfung (,sehr gut")

Diplomarbeit am Dritten Physikalischen Institut der Universität Göttingen unter Anleitung von Prof. Dr. D. Ronneberger. Titel der Arbeit: „Instationäre Strömungsablösung an der Hinterkante einer Splitterplatte“

Hauptstudium (Physik) an der Georg-August-Universität in Göttingen

Physikstudium an der Universität Aarhus, Dänemark über das Erasmus/Sokrates-Programm

Grundstudium (Physik) an der Georg-August-Universität in Göttingen

Zivildienst im Kreiskrankenhaus Mellrichstadt

Abitur

Rhön-Gymnasium Bad Neustadt a. d. Saale

Grundschule Brendlorenzen 
